\title{
9.M. Ollen
}

DIE FLEDERMÄUSE DES BERLINER MUSEUMS FÜR NATURKUNDE.

1. LIEFERU N G.

DIE

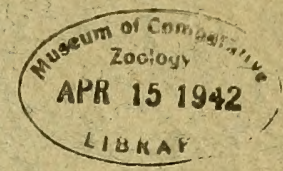

M E G A C H I R O P T E R A

DES BERLINER MUSEUMS FÏR NATURKUNDE.

BEARBEITET UND DURCH 2 VERBREITUNGSKARTEN UND

BESTIMMUNGSTABELLEN FÜR ALLE BEKANNTEN ARTEN ERGÄNZT

\section{PAUL MATSCHIE,}

KUSTOS AM MUSEUM FÜR NATURKUNDE ZU BERLIN.

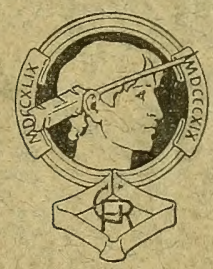

B E R L I N.

DRUCK UND VERLAG VON GEORG REIMER. 
HARVARD UNIVERSITY

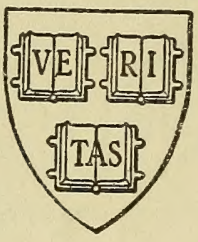

LIBRARY OF THE MUSEUM OF COMPARATIVE ZOÖLOGY

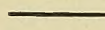

GIFT OF

GLOVER M. ALLEN 
DIE

\title{
F L E D E R M Ä U S E \\ DES
}

\section{BERLINER MUSEUMS FÜR NATURKUNDE.}

\author{
NEUNZIG
}

UNTER LEITUNG VON PROF. W. PETERS UND PAUL MATSCHIE GEZEICHNETE UND LITHOGRAPHIRTE TAFELN.

\section{BEARBEITET UND DURCH VERBREITUNGSKARTEN UND}

BESTIMMUNGSTABELLEN FÜR ALLE BEKANNTEN ARTEN ERGÄNZT

vON

\section{PAUL MATSCHIE,}

\section{KUSTOS AM MUSEUM FÜr NATURKUNDE ZU BERLIN.}

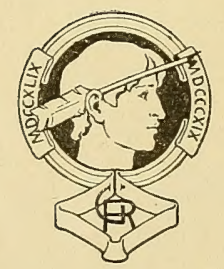

B E R L I N.

DRUCK UND VERLAG VON GEORG REIMER. 1899. 


\title{
DIE FLEDERMÄUSE DES BERLINER MUSEUMS FÜR NATURKUNDE.
}

\section{LIEFER U N G.}

DIE

\section{E G A C H I R O P T E R A}

\section{DES BERLINER MUSEUMS FÜR NATURKUNDE.}

\author{
VIERZEHN
}

UNTER LEITUNG VON PROF. W. PETERS UND PAUL MATSCHIE

GEZEICHNETE UND LITHOGRAPHIRTE TAFELN.

\section{BEARBEITET UND DURCH 2 VERBREITUNGSKARTEN UND}

BESTIMMUNGSTABELLEN FÜR ALLE BEKANNTEN ARTEN ERGÄNZT

\section{PAUL MATSCHIE,}

KUSTOS AM MUSEUM FÜR NATURKUNDE ZU BERLIN.

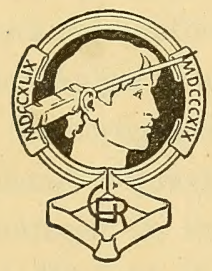

B E R L I N.

DRUCK UND VERLAG VON GEORG REIMER. 1899. 
LIBRARV 


\section{$\cdots$ \\ Vorwort.}

Professor Dr. Peters, 1857-1883 Direktor des Berliner Zoologischen Museums, hatte beabsichtigt, unter dem Namen: Museum Zoologicum Berolinense. Chiroptera eine Monographie der Fledermäuse herauszugeben. Während der Jahre 1865-1883 sind für diesen Zweck 75 lithographirte Tafeln durch die bekannten, inzwischen verstorbenen Thiermaler Franz Wagner und Gustav Mützel hergestellt worden. Im Frühjahr 1883 starb Peters und in seinem Nachlasse fand sich keinerlei Manuskript über Fledermäuse. Die Verlagsbuchhandlung von Georg Reimer, welcher die Tafeln gehören, bemühte sich Jahre lang vergeblich, einen Herausgeber für die werthvollen Abbildungen zu finden. Erst jetzt, 34 Jahre nach der Herstellung der ersten Tafel, erscheint der erste Theil des Werkes.

Die Verlagsbuchhandlung hat in ausserordentlich weitgehender Weise dafür gesorgt, dass die vorliegenden Tafeln soweit ergänzt werden konnten, wie der heutige Stand unserer Kenntniss der Fledermäuse es verlangt. Nicht weniger als 15 neue Tafeln sollen zu den schon vorhandenen 75 treten; 11 von ihnen wurden gezeichnet und lithographirt durch meine Frau Anna, geb. Held, und dürfen sich gleichwerthig den von Wagner und Mützel gearbeiteten an die Seite stellen. Der Tod hat meine Frau vèrhindert, ihr Werk zu vollenden.

Bei der Aufgabe, einen Text zu diesen Tafeln zu schreiben, habe ich darauf verzichten müssen, eine Monographie der Fledermäuse zu schaffen, weil das im Berliner Museum für Naturkunde aufbewahrte Material nicht ausreicht für die Bearbeitung dieser nicht leichten Aufgabe, und ein längerer Aufenthalt in Paris, London und Leyden zum Studium der dortigen Sammlungen mir nicht möglich war.

Ich habe mich deshalb damit begnügt, nach zoogeographischen Gesichtspunkten die Arten, welche mir vorlagen, in Gruppen zusammenzustellen, die von mir nicht untersuchten Arten nach Möglichkeit neben die am nächsten verwandten Formen einzureihen, in Bestimmungstabellen die Unterschiede anzugeben, welche ein Erkennen der einzelnen Arten erleichtern, und die im Berliner Museum vorhandenen Exemplare aufzuzählen. 
Besonderen Werth habe ich auf eine genaue Feststellung der Fundorte gelegt.

Die Litteratur wurde im allgemeinen nur insoweit genannt, als sie nicht in Trouessart's und Dobson's Werken zu finden ist.

Durch die Liebenswürdigkeit des Herrn Geheimen Hofraths Dr. A. B. Meyer, Direktor des Zoologischen Museums in Dresden, ist es mir vergönnt gewesen, einen Theil des Dresdener Materials an Flughunden hier in Berlin untersuchen zu dürfen.

Mit seiner Bewilligung habe ich diese Exemplare in meiner Arbeit aufgeführt.

Ich sage hier Herrn Geh. Hofrath Dr. A. B. Meyer meinen herzlichsten Dank. Auch Herrn Dr. Jentink, Direktor des Leydener Museums, habe ich für eine freundliche Auskunft auf eine Frage zu danken, vor allem aber meinem verehrten Herrn Chef, Geh. Regierungsrath Professor Dr. K. Möbius, welcher mich in weitgehender Weise unterstützte.

Den ersten Theil meiner „Fledermäuse des Berliner Museums für Naturkunde" lege ich hiermit vor. Er umfasst die Megachiroptera. In 4 Lieferungen soll der gesammte Stoff bewältigt werden. Eine allgemeine Einleitung in die Fledermauskunde werde ich der letzten Lieferung beigeben.

Berlin, am 14. Mai 1899.

Paul Matschie.

\section{Verzeichniss der Abkürzungen.}

B. M. = Museum für Naturkunde zu Berlin.

Dresd. M. = Dresdener Museum.

W.-Afrika $=$ Atlantische Küste von der Wasserscheide zwischen dem Senegal und Gambia nach Süden bis zur Wasserscheide südlich vom Catumbella, landeinwärts bis zu der Wasserscheide, auf welcher die Niger-Zuflüsse entspringen, Niger bis ungefähr zur Benue-Mündung, Gebiet der Küstenflüsse von Nieder-Guinea und Gebiet des Congo.

O.- u. S.-Afrika $=$ Afrika südlich von der Sahara, mit Ausnahme des unter "West-Afrika“ umschlossenen Gebietes.

Madagass. Geb. = Madagaskar, Maskarenen, Komoren, Rodriguez, Aldabra-Inseln und Seychellen. 


\section{Arten-Verzeichniss der ersten Lieferung.}

\section{Ordnung: Chiroptera.}

\section{Unterordnung I: Megachiroptera.}

\section{Familie: Pteropodidae.}

Seite

1. Pteropus Briss. . . . . . . 5

a. A c er odon Jourd. . . . . . . 9

1. jubatus Eschsch. . . . . 9

2. macklotii Temm. . . . . . 9 celebensis Schleg. . . . 10 batchianus Gray . . . . 10 macklotii Temm. . . . . 10 floresii Gray . . . . . 11

b. Pteralopex Thos. ...... 11

1. atrata Thos........ 11

c. Eunycteris Gray. . . . . 11

1. melanopogon Schleg. Ptrs. . . 11 melanopogon Schleg. Ptrs. . 11 papuanus Ptrs. Dor. . . . . 12 degener Ptrs. . . . . . 12 neohibernicus Ptrs. . . . 12

d. Pteropus Briss. s. str. . . . 12

1. chrysoproctus Temm. . . . 14

2. aruensis Schleg. Ptrs. . . . 14

3. keyensis Schleg. Ptrs. . . . 14

4. coronatus Thos. . . . . 14

5. grandis Thos. . . . . 15

6. celaeno Herm. . . . . . 15

7. edwardsi Geoffr. . . . . 15 edwardsi Geoffr. ..... 15 seychellensis A. M.-E. . . 16 aldabrensis True . . . . 16

8. medius Temm....... 16

9. nicobaricus Fitz. . . . . 16 nicobaricus Fitz. . . . 16 modiglianii Thos. ... 17 aterrimus Temm. . . . 17

10. alecto Temm. . . . . . 17

11. chrysauchen Ptrs. . . . . 18

12. conspicillatus Gould . . . . 18

13. gouldii Ptrs......... . 18

14. tonganus Q. G...... . 19

e. Spectrum Gray . . . . . . . 19

1. poliocephalus Temm. . . . 21

2. vetulus Jouan ...... 21

3. anetianus Gray . . . . . 22

4. samoensis Peale . . . . . 22
5. rayneri Gray Seite

6. brunneus Dobs. . . 29

7. scapulatus Ptrs. . . . . 22

8. macrotis Ptrs. . . . . . 23

9. epularius Rams. . . . . 23

10. hypomelanus Temm. . . . . 23

hypomelanus Temm. . . . 23

griseus Geoff. . . . . . 24

pallidus Temm. . . . . 24

ocularis Ptrs. . . . . . 24

tomesii Ptrs........ 25

vociferus Peale..... . 25

condorensis Ptrs. . . . . 25

fuscus Dobs........ 25

macassaricus Heude. . . 26

lombocensis Dobs. . . . 26

natalis Thos....... 26

11. assamensis $\mathrm{Mc} \mathrm{Cl}$. . . . 26

12. leucopterus Temm. . . . . 26

13. formosus Sclat. . . . . 27

14. loochoensis Gray . . . . . 27

15. dasymallus Temm. . . . . 27

16. pselaphon Lay . . . . . . 27

17. mariannus Desm. . . . . 27

18. insularis H. J. . . . . . 28

19. phaeocephalus Thos. . . . 28

20. ualanus Ptrs. . . . . . 28

21. admiralitatum Thos. . . . 28

22. tuberculatus Ptrs. . . . . 29

23. vampyrus L. . . . . . . 29

24. livingstonii Gray . . . . . 30

25. rodricensis Dobs..... . 30

f. Sericonycteris Mtsch. . . . 30

1. rubricollis Geoffr..... . 30

2. temmincki Ptrs. . . . . 31

temmincki Ptrs. . . . . 31

petersi Mtsch. ...... 31

heudei Mtsch. ...... 32

3. personatus Temm. . . . 32

4. capistratus Ptrs. . . . . 32

5. molossinus Temm. ... . 32

6. woodfordi Thos. . . . . 33

2. Stylocteninm Mtsch. .. . . . . 33

1. wallacei Gray ..... 33

3. Epomophorus Benn. . . . . . 34

a. Hypsignathus Allen ..... 42

1. haldemani Halow. .... 42 
b. Epomophorus Benn. s str.... 43

1. macrocephalus Ogilb. . . . . 44

2. büttikoferi Mtsch.. . . . . 45

3. zechi Mtsch. . . . . . 46

4. zenkeri Mtsch. . . . . . . 46

5. angolensis Gray . . . . . 47

6. spec. nov.? . . . . . . . 48

7. wahlbergi Sund. . . . . 48

8. crypturus Ptrs.. . . . . . 49

9. stuhlmanni Mtsch. .... 50

10. neumanni Mtsch. . . . . 50

11. minor Dobs........ . 51

12. schoensis Rüpp. . . . . . 53

13. labiatus Temm. . . . . 54

14. doriae Mtsch. ...... 54

15. anurus Hgl. . . . . . . 54

16. spec. nov.? . . . . . 55

17. gambianus 0 gilb..... . 55

c. Epomops Gray. . . . . 56

1. franqueti Tomes..... 56

2. comptus Allen...... . 57

3. dobsonii Boc. . . . . 57

d. Micropteropus Mtsch. ... 57

1. pusillus Ptrs. . . . . . 58

e. Nanonycteris Mtsch..... . 58

1. veldkampii Jent. . . . . . 59

4. Rousettus Gray . . . . . . . 59 (= Xantharpyia Gray.)

a. Pterocyon Ptrs. . . . . . 62

1. stramineus Geoffr. ..... 62

2. dupreanus Pollen...... 63

b. Myonycteris Mtsch. .... 63

1. torquatus Dobs. ..... 64

2. angolensis Boc. ..... 64

c. Rousettus Gray s. str. . . . 65

1. brachycephalus Boc. . . . 66

2. collaris Ill. . . . . . 66

3. aegyptiacus Geoffr. . . . 66

4. amplexicaudatus Geoffr. . . 67

5. brachyotis Dobs..... . 68

5. Boneia Jent. . . . . . . 69

1. bidens Jent. . . . . . . 69

2. menadensis Thos. . . . 69

6. Harpyionyeteris Thos. . . . . 70

1. whiteheadi Thos..... 70

7. Scotonycteris Mtsch. . . . . 70

1. zenkeri Mtsch....... 71

8. Cynopterus F. Cuv. . . . . . 71 a. Cynopterus F. Cuv. s. str. . . 74

1. sphinx Vahl. ...... 74

2. ceylonensis Gray . . . . . 74

3. marginatus F. Cuv. ... . 75
4. seherzeri 1 Sitze

5. andamanensis Dobs. . . . 75

6. brachysoma Dobs. . . . 75

7. montani Robin ..... 75

8. titthaecheilus Temm. . . 75

9. horsfieldi Gray . . . . . 76

10. brachyotis S. Müll. . . . . 76

11. luzoniensis Ptrs. . . . . 76

12. grandidieri Ptrs. . . . . 76

b. Thoopterus Mtsch. . . . . 77

1. nigrescens Gray. . . . . 77

2. melanocephalus Temm. . . 77

3. blanfordi Thos. . . . . 77

9. Ptenochirus Ptrs.... . . . . 77

a. Ptenochirus Ptrs. s. str. . . 78

1. jagori Ptrs. . . . . 79

2. lucasi Dobs. . . . . 79

b. Megaerops Ptrs. . . . . 79

1. ecaudatus Temm. . . . 79

10. Balionycteris Mtsch. . . . . 80

1. maculata Thos. . . . . 80

11. Gelasinus Temm. . . . . . 81

a. Gelasinus Temm. s. str. . . 83

1. cephalotes Pall. . . . . 83

b. Bdelygma Mtsch. . . . . . 84

1. major Dobs. . . . . . 84

12. Leiponyx Jent. . . . . . . . 85

1. büttikof̂eri Jent. . . . . . 85

13. Cephalotes Geoffr. . . . . . 85

1. palliatus Geoffr.. . . . . 87

14. Notopteris Gray . . . . . . 88

1. macdonaldi Gray .... . 88

15. Eonycteris Dobs. . . . . . . 89

1. spelaea Dobs....... . 89

16. Callinycteris Jent. . . . . . 90

1. rosenbergi Jent. . . . . 91

17. Nesonycteris Thos. . . . . . 91

1. woodfordi Thos....... 91

18. Melonycteris Dobs. . . . . . 92

1. melanops Dobs...... . 93

19. Macroglossus F. Cuv. . . . . . 93

a. Macroglossus F. Cuv. s. str. . 95

1. minimus Geoffr. . . . . 96

2. lagochilus Mtsch. .... . 97

3. nanus Mtsch....... . 98

b. Syconycteris Mtsch. .... 98

1. australis Ptrs....... . 99

2. papuanus Mtsch. .... . 99

3. finschi Mtsch. . . . . . 100

4. crassus Thos....... 100

20. Megaloglossus Pgst.. . . . . 101

1. woermanni Pgst. . . . 101 


\section{Chiroptera.}

Säugethiere, deren vordere Extremitäten in Flugorgane umgewandelt sind. Die Nittelhand- und Fingerknochen mit Ausnahme des Daumens sind stark verlängert und durch Flughäute unter einander, mit dem Unterarm, den Körperseiten und mit den hinteren Extremitäten verbunden. Auch zwischen den Hintergliedmassen findet sich häufig eine Flughant. Der Daumen trägt stets eine Kralle. Zwei Zitzen an der Brust. Im Gebiss sind Incisivi, Canini, Praemolares und Molares vorhanden. Brustbein mit Kamm. Schlüsselbeine sehr kräftig.

\section{Unterordnung: Megachiroptera.}

Der Rand des Ohres bildet einen ovalen Ring dadurch, dass sich der Aussen- und Innenrand vor der Ansatzstelle des Ohres am Kopfe berühren. Molaren mit stumpfen Höckern und mit einer Längsfurche. Der knöcherne Gaumen reicht über den letzten Molar hinaus.

\section{Unterordnung: Microchiroptera.}

Der Aussenrand des Ohres entspringt an einer anderen Stelle der Kopfhaut als der Innenrand; Molaren spitzhöckerig oder mit schneidenden Rändern, niemals mit einer Längsfurche; der knöcherne Gaumen ist nicht über den letzten Miolaren hinaus verlängert.

\section{Unterordnung: Megachiroptera.}

Nur eine Familie mit den Merkmalen der Unterordnung.

\section{Familie: Pteropodidae.}

Die Familie Pteropodidae umfasst augenblicklich 20 Gattungen mit ungefähr 145 Arten resp. Abarten, welche ich in 35 Untergattungen vertheilt habe.

Sie sind verbreitet über die tropischen und subtropischen Gegenden der alten Welt; man kennt sie aus dem madagassischen, aethiopischen und indischen Gebiet, aus dem südöstlichen Mitttelmeer-Gebiet und dem westlichen australisch-polynesischen Gebiet. Ihre nördlichsten Fundorte sind Cypern und die Riu-Kiu-Inseln. In Polynesien scheinen sie nicht östlich von den Carolinen, Salomon- und Samoa-Inseln und südlich von Australien und Neu-Caledonien vorzukommen.

Die grösste Mannigfaltigkeit an Arten weisen die Pteropodidae im malayischen Archipel auf z. B. auf Celebes, wo nicht weniger als 13 Arten neben einander leben. Eigenthümliche Gattungen treten nur in Westafrika, im hinterindischen Gebiet, und im australisch-polynesischen Gebiet auf. 


\section{Uebersicht der Gattungen:}

Die Ränder der Nasenlöcher sind nicht röhrenförmig verlängert, sondern springen höchstens am Innenrande etwas vor:

Mit einer Kralle oder einem Nagel am Zeigefinger:

Flughaut an die 1. oder an die 2. Zehe oder aber an die 1. und 2. Zehe angeheftet:

Flughaut an die 1. und 2. Zehe oder an die 2. Zehe angeheftet; Schnauze nicht auffallend verschmälert; Zunge nicht auffallend lang:

Schwanz unter der Behaarung versteckt oder nicht deutlich:

4 oder 5 Backenzähne im Oberkiefer; kein Büschel weisser Haare am Ohr:

4 Schneidezähne im Unterkiefer 2 Schneidezähne im Unterkiefer:

4 Schneidezähne im Oberkiefer . . . . . . Styloctenium Mtsch. p. 33

2 Schneidezähne im 0ber-

kiefer . . . . . Harpyionycteris Jent. p. 70

3 Backenzähne im Oberkiefer; vor und hinter der Ohrbasis je ein

Büschel weisser Haare. . . . Epomophorus Benn. p. 34

Schwanz nicht von der Behaarung: verdeckt:

4 Schneidezähne im Oberkiefer 2 Schneidezähne im Oberkiefer

Pteropus Briss. p. 5

Rousettus Gray p. 59

Boneia Jent. p. 69

Flughaut an die erste Zehe angeheftet:

4 Schneidezähne im Unterkiefer:

3 obere Molaren . . . . Scotonycteris Mtsch. p. 70

4 obere Mfolalen . . . Cynopterus F. Cuv. p. 71

2 Schneidezähneim Unterkiefer:

4 obere Molaren . . . . Ptenochirus Ptrs. p. 77

5 obere Molaren . . . . Balionycteris Mtsch. p. 80 
Flughaut an die 2. und 3. Zehe oder an die 3. Zehe; oder an die 4. Zehe angeheftet. Schnauze sehr lang und schmal; Zunge auffallend lang:

Muffel am Lippenrande nicht verbreitert:

Flughaut an die Basis der 4. Zehe angeheftet . . . . . . . Macroglossus F. Cuv. p. 93

Flughaut mit 2 Fältchen an die Basis der 2. und 3. Zehe angeheftet . . Megaloglossus Pgst. p. 101

Muffel am Lippenrande viel breiter als auf der Mitte der Oberlippe:

Flughaut an die Basis der 3. Zehe angeheftet .

Melonycteris Dobs. p. 92

Ohne Kralle oder Nagel am Zeigefinger:

Unterarm kürzer als 100 Millimeter; Schnauze sehr lang und schmal; Zunge auffallend lang:

Schwanz nicht sichtbar oder viel kürzer als der Kopf:

Schwanz nicht sichtbar. . .

Schwanz kurz, aber deutlich:

6 untere Backenzähne; Flughaut an die Basis der 1.Zehe angeheftet

5 untere Backenzähne; Flughaut an die Basis der 2.Zehe angeheftet

Nesonycteris Thos. p. 91.

Eonycteris Dobs. p. 89.

Callinycteris Jent. p. 90

Schwanz viel länger als der Kopf, die Flughaut ist an der Rückenmitte angewachsen und bedeckt den ganzen Rücken . . . . . . . . . . Notopteris Gray p. 88

Unterarm länger als 100 Millimeter; Schnauze nicht auffallend verschmälert; Zunge nicht auffallend lang:

Die an der Rückenmitte angewachsene Flughaut bedeckt den Rücken vollständig . .

Die Flughaut ist an die Seiten des Rückens angeheftet . . Leiponyx Jent. p. 85

Nasenlöcher in röhrenförmigen Hautvorspringen. . '. . , Gelasinus Temm. p. 81. 


\section{Uebersicht der geographischen Verbreitung.}

\begin{tabular}{|c|c|c|c|c|}
\hline & $\begin{array}{l}\text { Epomophorus } \\
\text { Pteropus }\end{array}$ & Rousettus & $\begin{array}{l}\text { Notopteris } \\
\text { Scotonycteris } \\
\text { Cynopterus }\end{array}$ & $\begin{array}{c}\text { Harpyionycteris } \\
\text { Styloctenium } \\
\text { Balionycteris }\end{array}$ \\
\hline West-Afrika & Epomophorus & Rousettus & Scotonycteris & - \\
\hline S. u. 0.-Afrika & Epomophorus & Rousettus & - & - \\
\hline Madagass. Gebiet & Pteropus & Rousettus & - & - \\
\hline Oestl. Mittelmeer-Geb. & - & Rousettus & - & - \\
\hline Vorder-Indien & Pteropus & Rousettus & Cynopterus & - \\
\hline Hinter-Indien & Pteropus & Rousettus & Cynopterus & - \\
\hline Grosse-Sunda-Inseln & Pteropus & Rousettus & Cynopterus & Balionycteris \\
\hline Philippinen & Pteropus & Rousettus & Cynopterus & Harpyionycteris \\
\hline Celebes & Pteropus & Rousettus & Cynopterus & Styloctenium \\
\hline Molukken & Pteropus & Rousettus & Cynopterus & $一$ \\
\hline Neu-Guinea & Pteropus & Rousettus & - & - \\
\hline Bismarck - Archipel & Pteropus & Rousettus & 一 & - \\
\hline Salomon - Inseln & Pteropus & Rousettus & - & - \\
\hline Uebriges Melanesien & Pteropus & - & Notopteris & - \\
\hline Samoa & Pteropus & - & - & - \\
\hline Micronesien & Pteropus & - & Notopteris & - \\
\hline Australien & Pteropus & - & - & - \\
\hline
\end{tabular}

\begin{tabular}{|c|c|c|c|c|}
\hline & $\begin{array}{c}\text { Leiponyx } \\
\text { Cephalotes } \\
\text { Boncia }\end{array}$ & $\begin{array}{c}\text { Plenochirus } \\
\text { Gelasinus }\end{array}$ & $\begin{array}{l}\text { Macroglossus } \\
\text { Megaloglossus }\end{array}$ & $\begin{array}{l}\text { Eonycteris } \\
\text { Melonycteris } \\
\text { Callinycteris } \\
\text { Nesonycteris }\end{array}$ \\
\hline West-Afrika & Leiponyx & - & Megaloglossus & - \\
\hline Hinter-Indien & - & $\rightarrow$ & Macroglossus & Eonycteris \\
\hline Grosse-Sunda-Inseln & - & Ptenochirus & Macroglossus & Eonycteris \\
\hline Philippinen & - & Ptenochirus & Macroglossus & - \\
\hline Celebes & Boneia, Cephalotes & Gelasinus & Macroglossus & Callinycteris \\
\hline Molukken & Cephalotes & Gelasinus & Macroglossus & - \\
\hline Neu-Guinea & Cephalotes & Gelasinus & Macroglossus & Melonyeteris \\
\hline Bismarck - Archipel & Cephalotes & Gelasinus & Macroglossus & Melonycteris \\
\hline Salomon-Inseln & Cephalotes & Gelasinus & Macroglossus & Nesonycteris \\
\hline Australien & - & Gelasinus & Macroglossus & - \\
\hline
\end{tabular}




\title{
1. Genus. Pteropus Brisson.
}

\author{
1756 Pteropus Brisson, Quadrup. p. 153.
}

1869. I. J. Fitzinger in Sitzb. Ak. Wiss. Wien LX, 1. Abth. p. 13-71. 1878. G. E. Dobson, Catalogue of the Chiroptera p. 15-70, 552, Tafel III und IV und 1880 in Rep. British Assoc. 1880 p. 4-5. - 1887. F. A. Jentink in Mus. d'Hist. Nat. Pays-Bas IX p. 252-263 und 1888 l. c. XII p. 138-150. - 1897. E. L. Trouessart, Cat. Mamm. Nov. Ed. I. p. 77-83.

Grosse Fledermänse ohne Schwanz mit einer Kralle am Zeigefinger. Der $\mathrm{R}$ and des $\mathrm{Ohres}$ bildet einen ovalen Ring, da sich der Aussenrand mit dem Innenrand vor der Ansatzstelle des Ohres an den Kopf berührt. Die Flughaut setzt sich an den Rücken der zweiten Zehe an. Schnauze hundeartig. Der Metacarpus des Mittelfingers ist kürzer als der Zeigefinger; der Daumen ist nicht von der Flughaut umhüllt; Gebiss $\frac{4,1,3,2}{4,1,3,3}$. Die oberen Schneidezähne sind von den Eckzähnen durch eine Lücke getrennt und stehen bogenförmig; der erste obere Praemolar ist sehr klein und fehlt häufig den ausgewachsenen Thieren. Backenzähne stumpfhöckerig mit einer Längsfurche auf der Krone. Zwischen dem Eckzahn und dem 2. Praemolar ist eine Lücke, welche bei vielen Arten desto breiter erscheint, je älter das Thier ist. Der knöcherne Gaumen reicht weit über die Molarenreihe hinaus. - Saugwarzen unter der Achselgrube; Ruthe des Männchen mit Penesknochen. An den Halsseiten sind bei vielen Arten Haarbüschel entwickelt, welche namentlich bei alten Männchen besonders hervortreten. Diese Haarbüschel stehen mit Drüsen in Verbindung, welche einen stark riechenden Saft absondern. Die Nasenöffnung ist wulstig umrandet; zwischen den Nasenlöchern verläuft eine tiefe Furche über die Oberlippe herab.

Die Flughunde sind Baumbewohner, leben vorwiegend von Frïchten, fressen aber auch Insekten und Vogeleier. Nanche Arten leben in ungeheuren Schaaren zur Fortpflanzungszeit zusammen und haben dann ihre gemeinschaftlichen Schlafplätze, von denen aus sie $\mathrm{zu}$ den von ihnen bevorzugten Nahrungsbäumen fliegen. $O b$ alle Arten regelmässige Wanderungen unternehmen oder nur umherstreichen, das ist noch nicht festgestellt. Sie trinken gern, indem sie dicht über der Wasserfläche dahin fliegen. Das Fleisch der Flughunde soll gut schmecken.

Ungefähr 110 Arten sind bis jetzt beschrieben; von diesen hat Trouessart im Jahre 1897,53 als Arten und 9 als Unterarten aufgeführt, während die übrigen unter die Synonyme verwiesen worden sind. Jourdan hat im Jahre 1837 (F. Cuvier, Ann. Sc. Nat. Ser. 2. VIII. 1837 p. 369) Pteropus pyrocephalus Meyen = jubatus Eschsch. zum Typus einer neven Gattung Acerodon erhoben. Gray hielt diese Gattung nicht aufrecht, sondern trennte die Arten von Pteropus unter drei Untergattungen: Spectrum, Pteropus und Eunycteris und erhob Pt. pselaphon (wahrscheinlich durch ein Versehen des Setzers) zu einer neuen Gattung: 
Pselaphon. (Cat. Monk. Lem. Fruit-eating Bats 1870, p. 100-113.) Fitzinger und Dobson zogen es vor, alle von ihnen angenommene Arten unter einem Gattungsnamen zu vereinigen. Oldf. Thomas errichtete (P. Z. S. 1888 p. 473 pl. XX Nr. 3 und pl. XXI Nr. 4-7) eine mit Pteropus nahe verwandte Gattung Pteralopex und endlich hat Père Heude (Mrém. conc. l'Hist. nat. Emp. Chin. III. 1896 p. 177) für die Aufrechterhaltung der Gattung Acerodon sich ausgesprochen und $P$ t. floresii Gray dazu gezogen.

Ich habe mich entschlossen aus zoogeographischen Gründen 6 Untergruppen anzunehmen:

In der ersten Gruppe befindet sich bis jetzt nur eine Art, Pteralopex atratus.

Als zweite Gruppe fasse ich Acerodon Jourd, auf und schliesse in dieser Gruppe die Arten Pt. jubatus Eschsch. und macklotii Temm. nebst seinen Verwandten zusammen. Die hier vereinigten Arten sind genügend durch ihre grossen ovalen, mit deutlichen vorderen Basalhöckern versehenen Molaren gekennzeichnet.

Eine dritte Gruppe bildet die Untergattung: Eunycteris Gray, für welche ich als Merkmale angebe: Rücken bei ausgewachsenen Thieren nackt, bei jungen Thieren noch ein śchmaler Strich auf der Rückenmitte behaart. Ohren breit und kurz. Unterschenkel niemals behaart; Flügel sehr nahe an der Rückenmitte angesetzt. Sehr grosse Arten. Hierher rechne ich: Pt.melanopogon Schleg., degener Ptrs., papuamus Ptrs. und Doria und neohibernicus Ptrs. Als Typus hat zu gelten: Pt. phaeops Gray nec. Temm. partim = Pt. melanopogon Schleg.

Als vierte Gruppe betrachte ich diejenigen kleinen Formen, deren Unterarm nicht länger ist als $120 \mathrm{~mm}$, und welche entweder dicht behaarte Unterschenkel haben oder ganz kurze, wenig aus dem Pelze hervorstehende Ohren.

Hierher gehören Pt. personatus Temm., temmincki Ptrs., capistratus Ptrs., woodfordi Thos., molossinus Temm. und mibricollis Geoffr. - Da für diese Gruppe ein Name nicht vorhanden ist, so nenne ich sie Sericonycteris Mtsch. subgen. nov. [von onpoxòv = Seide und v'xiepıs $=$ Fledermaus] und nehme als Typus: Pt. rubricollis Geoffr.

Zu der fünften Gruppe, Spectrum Gray, ziehe ich alle diejenigen Formen, welche nicht zu den bisher aufgeführten Gruppen gehören, und bei denen die Entferning vom vordern Augenwinkel zur Nasenspitze nicht grösser ist als die Breite der Schnauze an den Mundwinkeln. Bei den zu dieser Gruppe gehörigen Arten ist der vorletzte obere Molar deutlich nach hinten verschmälert. Die hierher zu rechnenden. Arten findet man weiter unten auf der Verbreitungstafel aufgeführt. Typus: Pt. vulgaris Geoffr. von Mauritius.

Die zur sechsten Gruppe zu ziehenden Arten, welche die Untergattung Pteropus Briss. s. str. bilden, haben eine lange Schnauze; bei ihnen ist die Entfernung vom vorderen Augenwinkel zur Nasenspitze bedeutend grösser als die Breite der Schnanze an den Nundwinkeln. Die hierher gehörigen Arten findet man ebenfalls auf der Verbreitungstafel aufgezählt. Typus: Pt. conspicillatus, Gould von Fitzroy Island.

Ich möchte hier auf die Wichtigkeit der Gaumenfalten für die Unterscheidung der einzelnen Gruppen hinweisen: das mir zu Gebote stehende. Material reicht für eine vollständige Betrachtung dieses Merkmals nicht aus. Ich glaube, dass jede Gruppe durch eine eigenthümliche Bildung der Gaumenfalten charakterisirt ist.

Hab. Von den Komoren nach Osten bis zu den Samoa-Inseln, vom Himalaya den Riu-Kiu-Inseln und Bonin-Inseln nach Säden bis Réunion im Indischen Ocean, bis Tasmanien, Neu-Caledonien und bis zu den TongaInseln im Stillen 0 cean. Nicht vertreten innerhalb der tropischen und subtropischen Geggenden der Alten Welt in Afrika, in Süd-Asien westlich von der indischen Wüste und nördlich von Himalaya, in Polynesien südlich vom Wendekreis des Steinbocks und östlich von den Karolinen, Salomons-Inseln und Samoa-Inseln. 


\section{Uebersicht der Untergattungen.}

Unterarm höchstens $12 \mathrm{~cm}$ lang. Wenn er länger ist als $11 \mathrm{~cm}$, so ist der Unterschenkel dicht behaart und im Gesicht ist eine Bindenzeichnung vorhanden . . . . . . .

Der Unterarm ist länger als $10 \mathrm{~cm}$ und wenn er kürzer als Sericonycteris Mtsch. p. 30 $12 \mathrm{~cm}$ ist, so sind die Unterschenkel nackt, die Ohren ragen mindestens $10 \mathrm{~mm}$ aus den Pelz hervor und im Gesicht ist eine helle oder dunkle Linienzeichnung nicht vorhanden:

Obere Caninen zweispitzig: . . . . . . . . . . Obere Caninen einspitzig, höchstens mit Basalhöckern .

Wangen sehr dicht mit langen nach hinten gerichteten, ziemlich starren Haaren bedeckt . . . . Wangen nicht auffallend lang behaart:

Rücken nackt oder (bei jungen Thieren) ein schmales spärlich behaartes Feld in der Rückenmitte, die Ohren sind kürzer als die Mundspalte : . . . . . . . . . . Pteralopex Thos. p. 11 Acerodon Jourd. p. 9 Rücken dicht, entweder mit glatt anliegenden oder wolligen Haaren bedeckt; wenn nur ein schmales Feld des Rückens behaart ist, so sind die Ohren mindestens so lang, wie die Mundspalte oder oben sehr breit abgerundet.

Die Entfernung zwischen dem vorderen Augenwinkel und der Nasenspitze ist viel breiter als das Gesicht an den Mundwinkeln: . . . . . . . . . . so breit wie das Gesicht an den Mundwinkeln: . . . . . . Spectrum Gray p. 19

\section{Uebersicht der geographischen Verbreitung.}

\begin{tabular}{c|c|c|c|c}
\hline & $\begin{array}{c}\text { Pteralopex } \\
\text { Eunycteris } \\
\text { Acerodon }\end{array}$ & Pteropus & Spectrum & Sericonycteris \\
\hline \hline Rodriguez & - & - & rodricensis & - \\
Maskarenen & - & - & vampyrus & rubricollis \\
Madagaskar & - & edwardsi & vampyrus & - \\
Komoren & - & edwardsi & livingstoni & - \\
Aldabra & - & aldabrensis & - & - \\
Seychellen & - & seychellensis & - & - \\
Ceylon & kelaarti & - & - \\
Vorderindien & - & medius & - & -
\end{tabular}




\begin{tabular}{|c|c|c|c|c|}
\hline & $\begin{array}{c}\text { Pteralopex } \\
\text { Eunycteris } \\
\text { Acerodon }\end{array}$ & Pteropus & Spectrum & Sericonycteris \\
\hline Hinterindien & - & celaeno & assamensis & - \\
\hline Andamanen, Nicobaren & - & nicobaricus & - & - \\
\hline Nias & - & nicobaricus & - & 一 \\
\hline Mentawei & - & celaeno & hypomelanus. & - \\
\hline Enghano, Cocos-Ins. & - & modiglianii & hypomelanus & - \\
\hline Bavean & - & aterrimus & - & - \\
\hline Sumatra & - & celaeno & - & - \\
\hline Christmas-Inseln & 一 & - & natalis & - \\
\hline Java & - & celaeno & $-:$ & - \\
\hline Bali, Lombock & - & celaeno & lombocensis & - \\
\hline Borneo & - & celaeno & tomesii & - \\
\hline Natuna-Inseln & - & celaeno & hypomelanus & - \\
\hline Mangsi & - & - & vociferus & - \\
\hline Pulo Condor & - & - & condorensis & - \\
\hline Philippinen & jubatus & celaeno & hypomelanus & - \\
\hline *Timor & macklotii & celaeno & griseus & temmincki \\
\hline Flores & floresii & - & - & heudei \\
\hline Celebes & celebensis. & alecto & hypomelanus. & personatus \\
\hline Halmahera-Gruppe & batchianus & chrysauchen & hypomelanus & personatus \\
\hline Amboina-Gruppe & melanopogon & chrysoproctus & ocularis u. pallidus & petersi \\
\hline Nord-Neu-Guinea & papuanus & chrysauchen & hypomelanus & - \\
\hline Süd- tund Südost-Neu-Guinea & papuanus & conspicillatus & epularius & - \\
\hline Aru-Inseln & degener & aruensis & macrotis & - \\
\hline Key-Inseln & - & keyensis & - & - \\
\hline $\begin{array}{l}\text { Bismarck-Archipel } \\
\text { Salomons-Inseln }\end{array}$ & $\begin{array}{c}\text { neohibernicus } \\
\text { atratus }\end{array}$ & $\begin{array}{l}\text { coronatus } \\
\text { grandis }\end{array}$ & $\begin{array}{l}\text { admiralitatum } \\
\text { rayneri }\end{array}$ & $\begin{array}{l}\text { capistratus } \\
\text { woodfordi }\end{array}$ \\
\hline Nord-Australien & - & gouldii & scapulatus & - \\
\hline Percy-Insel & - & gouldii & brunneus & - \\
\hline Südost-Australien & - & - & poliocephalus & $\dot{-}$ \\
\hline Neu-Caledonien & - & tonganus & vetulus & - \\
\hline Neu-Hebriden & - & tonganus & anetianus & - \\
\hline Fidschi, Samoa & - & tonganus & samoensis & - \\
\hline Ualan, Carolinen & - & - & ualanus & - \\
\hline Ponape - & - & - & ualanus (?) & molossinus \\
\hline Mortlock & - & - & phaeocephalus & molossinus \\
\hline Ruck & - & - & insularis & - \\
\hline Palau, Guam & - & - & mariannus & - \\
\hline Bonin-Inseln & - & - & pselaphon & - \\
\hline Nord-Riu-Kiu & - & - & dasymallus & - \\
\hline Süd-Riu-Kiu & - & - & loochoensis & - \\
\hline Formosa & - & - & formosus & - \\
\hline China (?) & - & - & leucopterus & - \\
\hline ? & - & - & tuberculatus & - \\
\hline
\end{tabular}

* A. F. de Seabra (Jorn. Scienc. Hath. Phys. Nat. Lisboa 2. ser. 1897 tom. V Nr. XVIII p. 117-125) führt ausserdem noch für Timor auf Grund der von ihm untersuchten Exemplare auf: Pt. poliocephalus, samoensis, brunneus, pselaphon, hypomelanus und caniceps. Eine Nachprüfung dieser Bestimmungen wird wahrscheinlich deren Unrichtigkeit ergeben. 


\section{Subgen. Acerodon Jourd.}

1837. Jourdan bei F. Cuvier, Ann. Sc. Nat. Ser. 2. VIII 1837 p. 369. 1896. Heude, Mém. Hist. Nat. Empire Chinois III p. 177-178.

Zähne sehr kräftig, kegelförmig; dritter oberer Praemolar und erster oberer Molar mit einem stark vorspringendem vorderen Höcker; erster unterer Molar mit einem hinterem und innerem, von dem zweihöckrigen Zahn abgeschnürten Wulst. Auf dem weichen Gaumen befinden sich hinter dem zweiten Praemolar eine ungetheilte Falte und dahinter mindestens 11 gezähnelte, in der Mitte getrennte Bogenfalten. Wangen dicht mit langen, nach hinten gerichteten Haaren besetzt.

Typus: Pt. jubatus. Eschsch. Uebersicht).

Hab. Philippinen, Djilolo, Batjan, Celebes, Flores, Timor (s. Verbreitungs-

Uebersicht der Arten:

Unterarm bei erwachsenen Thieren länger als $160 \mathrm{~mm} \ldots$ jubatus.

Unterarm kürzer als $150 \mathrm{~mm}$ : macklotii und Verwandte.

1. Pt. jubatus (Eschscholtz). 1831. Pt. jubatus Eschsch. von Manila, Eschscholtz Zool. Atl. IV. p. 1-2, t. 16 (Thier und Schneidezähne). - 1833. Pt. pyrrhocephalus Meyen von Manila, Nov. Act. Acad. Nat. Cur. V., XVI., P. II. p. 604 t. 45 (Thier), t. 46, f. 1-3 (Schädel). - 1837. Acerodon jubatus Jourdan bei F. Cuvier, Ann. Sc. Nat. Ser. 2, VIII. p. 369. - 1878. Pt.jubatus Dobson 1. c. p. 68-69. - 1890. Pt. spec. Nehring, Sitzb. Ges. nat. Fr. Berlin p. 102. - 1896. A. jubatus, Heude, Mém. Hist. Nat. Empire Chinois III. p. 177-178, t. V. f. 11 u. 12 (Gebiss). - 1897. Pt. jubatus, Trouessart, Cat. Mamm. I. p. 83.

Für diese Art scheint ein heller Fleck auf dem Hinterkopf, der sich bei ausgefärbten Thieren nach vorn bis zur Stirn und nach hinten bis zum Nacken erstreckt, sehr charakteristisch zu sein. Incisiven kegelförmig, Molarenreihe beim ausgewachsenen $\delta 25,5$, beim ausgewachsenen 우 $24 \mathrm{~mm}$ lang; Entfernung zwischen dem Vorderrande des Foramen lacrymale und dem Vorderrande der Nasalia kleiner als die äussere Entfernung der oberen vorletzten MIolaren.

Hab. Philippinen: Luzon, Dinagut, Mindanao (Dobson), Concepcion, Panay (Elliot, Field Columb. Mus. II. 1896 p. 76), Paragua, Jolo, Batangas (De Elera, Cat. System. 1895 p. 6).

[B. M.] $\delta^{\top}+$ Manila, Meyen; $\delta^{\nearrow}$ pull. Negros, A. B. Meyer; $\delta^{\nearrow}+$ San Fernando, Luzon, Jagor. - - [IIuseum der landwirthschaftl. Hochschule Berlin]

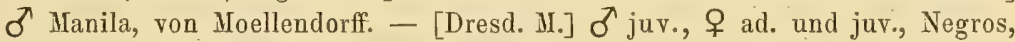
A. B. Meyer. - Davao, Mindanao, Platen.

Pt. aurinuchalis Elliot. 1896 Mai, Field Columbian Museum II. Zoolog. Ser. vol. 1, Nr. 3, p. 77-78, t. XII (Schädel) von Ley te und Pt. lucifer Elliot 1. c. p. 78-79, t. XIII (Schädel) von Concepcion Panay scheinen sehr nahe mit jubatus verwandt zu sein. Aus den Beschreibungen vermag ich Unterschiede von jubatus für diese neuen Arten nicht zu erkennen.

2. Pt. macklotii (Temminck). - 1837. Pt. macklotii Temminck von Timor; Monogr. Mammal. II. p. 69 t. 35 f. 5 (Kopf). - (?) 1867. Pt. celebensis Schlegel bei Peters von Celebes, M. B. Akad: Berl. p. 333. - (?) 1870. Pt. floresii 
Gray von Flores und batchiana Gray von Batjan, Cat. Monk. Lem. Fruiteating Bats. p. 106 und 110. - (?) 1878. Pt. caniceps Dobson von Batjan, Cat. Chir. p. 68. - (?) 1896. Acerodon floresianus Heude von Flores, Mém. Hist. Nat. Empire Chinois III. p. 178 Anmerk. t. V. f. 10 (Gebiss). - 1897. Pt. mackloti Trouessart 1. c. p. 83.

Erinnert in der allgemeinen Färbung an Pt. hypomelamus, ist aber sehr leicht zu unterscheiden durch die grossen Ohren (25 mm lang) und die langen, straffen Haare an den Kopfseiten. Jentink zieht (Weber, Zool. Ergebn. I. 1. 1890 p. 126) Pt. macklotii mit Pt. celebensis zusammen. Die von mir untersuchten Exemplare von Celebes haben sämmtlich breitere Ohren als je ein Exemplar aus Timor und Flores, welche mir zu Gebote stehen. Das Timor-Exemplar stimmt auch in der Färbung nicht zu dem Flores-Exemplar, obwohl beide Männchen sind. Das o aus Flores ist grösser als dasjenige aus Timor, hat einen längeren Kopf, dunkle Kehle und strohgelbe Haare zwischen dem dunkelgraubraunen Haarkleide des Bauches; das $\sigma^{7}$ aus Timor hat kürzeren Kopf, bei ihm ist die Kehle nicht auffallend dunkel und der Bauch ist dunkelrostgraubraun.

Wie sich $P$. caniceps zu macklotii verhält, vermag ich vorläufig nicht zu entscheiden.

Wir besitzen durch Herrn von Martens ein Exemplar von Batjan, welches die langen Ohren und die langen Wangenhaare von macklotii besitzt, in der Gestalt der Molaren aber sehr an hypomelamus erinnert.

Im Schädelban sind die hierher gehörigen Formen durch den kurzen Schnauzentheil, die breiten oberen Molaren und die bei den Gattungsmerkmalen angegebenen Charaktere zu erkennen.

Hierher sind also zu stellen:

2a. Pt. celebensis Schlegel. - 1867. Pt. celebensis Schlegel bei Peters von Celebes, M. B. Akad. Berl. p. 333.

Hab. Celebes (Dobson l. c. p. 67). Celebes, Gorontalo, Soela Mangoeli (Jentink, Mus. D'Hist. Nat. Pays Bas. IX. Cat. Osteol. 1887 p. $262-263$ und XIl. 1888 p. 150). - Maros bei Makassar, Central-Celebes, Insel gegenüber Palopo, Luwu, Celebes (Jentink, Weber's Zool. Erg. 1890 I. 1 p. 126).

Abbildung des Schädels eines $f$ von Celebes auf Tafel [4] Fig. 7 mit den Gaumenfalten und eines zweiten Exemplars als Pt. macklotii auf Taf. [6] Fig. 6.

[B. MI.] 우 Celebes, Leyd. Mus.; $\sigma^{7}$ ㅇ. Soela Mangoeli, Bernstein. - [Dresd. M.] Gorontalo auf Celebes, Riedel. - [Sarasin-Samml.] $q$ Masarang und Tomohou auf Celebes, Gebr. Sarasin.

2b.Pt. batchianus Gray. - 1870. Pt. machlotii var. batchiana Gray von Batjan, Cat. Monk. Lem. Fruit-eating Bats. p. 110. - (?) 1878. Pt. caniceps Dobson von Batjan, Cat. Chir. p. 68.

Hab. Batjan, Djilolo (Dobson).

Abbildung des Schädels eines q juv. von Batjan auf Taf. [5], Fig. 13-14 als $P$ t. caniceps.

[B. MI.] $\&$ juv. Batjan, von Martens.

2c. Pt. macklotii Temminck. - 1837. Pt. macklotii Temminck von Timor, Monogr. Mammal. II. p. 69 t. 35 f. 5 (Kopf).

Hab. Timor.

[B. M.] ठ゙ Timor, Müller und Macklot. — [Dresd. M.] Timor durch Frank. 
2d. Pt. floresii Gray - 1870. Pt. floresii Gray von Flores, Cat. Monk. Lem. Fruit-eating Bats p. 106. - 1896. Acerodon floresianus Heude von Flores, Mém. Hist. Nat. Emp. Chinois III. p. 178, Anmerkung t. V. f. 10 (Gebiss).

[B. M.] $\sigma^{\lambda}$ Flores, Semmelinck.

Jentink erwähnt (Weber's Zoolog. Erg. I. 1. 1890 p. 126) Pt. mackloti von Sikka auf Flores, Weber (1. c. I. 1. 1890 p. 96 und III. 1. 1893 p. 268) ebenfalls von Flores. In Dobson's Catalog wird Pt. macklotii neben $P$. caniceps $=$ batchianus auch für Batjan aufgeführt nach einem von Wallace gesammelten Exemplar.

Dobson (1. c. p. 64) und nach ihm Trouessart (1. c. p. 83) ziehen Pt.vociferus Peale mit einem Fragezeichen zu Pt. macklotii. Sie folgen in dieser Auffassung J. Cassin, der U. S. Explor. Exp. 1858 Nammalogy p. 10-11 zuerst die von Peale (Zool. U. S. Explor. Exp. Vincennes. Quadrupeds p. 19. 1. ed. 1848) aufgestellte Art zu macklotii gezogen hatte. Nun liegt aber der Fundort, die Insel Mangsi in der Strasse von Balabak ganz ausserhalb des bisher bekannten Verbreitungsgebietes von macklotii, die Länge der Ohren, die Gestalt der Zähne, die Färbung und die Grösse des offenbar jungen Thieres spricht eher für die Zugehörigkeit von Pt. vociferus zur Untergattung Spectrim (vergl. p. 25).

\section{Subgen. Pteralopex Thos.}

1888. Oldf. Thomas, Ann. Mag. N.H. (6.) I p. 155; P. Z. S. p. 473-475, t. XX f. 3 (Kopf), t. XXI, f. 4-7 (Schädel). - 1897. Trouessart I. c. p. 83.

Obere Caninen mit 2 scharfen conischen Basalhöckern und zweispitziger Krone; Praemolaren und Molaren nicht mit einer Längsgrube, sondern vielspitzig.

Hab. Aola, Salomons-Inseln.

Nur eine Art: Pteralopex atrata Thos.

\section{Subgen. Eunycteris Gray.}

1870. Eunycteris Gray partim, Cat. Monk. Lem. Fruit-eating Bats p.112-113.

Sehr grosse Arten, deren Ohren kürzer als die Mundspalte sind. Rücken nackt oder (bei jungen Thieren) mit einem schmalen spärlich behaarten Längsfelde. Jochbogen unter dem Auge eben so breit wie in der Schläfengegend.

Typus: Pt. melanopogon (Schleg.) Ptrs.

Guinea, Bismarck-Archipel.

Hab. Sanghir-Inseln, Siao (nach Jentink), Amboina-Gruppe, Aru, Key, Neu-

Es ist mir vorläufig unmöglich, zu entscheiden, ob und wieviel geographische $\mathrm{Ab}$ arten von $P$ t. melanopogon unterschieden werden müssen, da das mir zur Verfügung stehende Material nicht ausreicht, und ich nicht weiss, wie diese Flughunde in den verschiedenen Jahreszeiten sich verändern und ob sie wandern.

1a. Pt. melanopogon (Schlegel) Peters. - 1867. Pt. melanopogon Schlegel bei Peters, M. B. Akad. Berlin p. 330. - 1837. Pt. phaeops Temminck von Amboina. Monogr. Mamm. II. p. 65 (exclus. I. p. 178) t. 35 f. 3 (Kopf), t. 36 f. $1-3$ (Schädel). - 1869. Fitzinger l. c. p. 34-36. - 1878. Dobson l. c, 
p. 44. - 1887. Jentink l. c. p. $255-256$ - - 1888. l. c. p. $142-143$ - - 1897. Trouessart 1. c. p. 80.

Hab. Sanghir-Inseln, Siao, Bonao, Buru, Amboina, Ceram, Saparua, Goram, Manavolka, Mysol (Jentink 1888).

[B. M.] $3 \delta^{\nearrow} \sigma^{\nwarrow}$ Amboina (Forsten, S. Müller) $\sigma^{\nwarrow}$ juv. Buru durch Frank.

1b. Pt. papuanus Peters u. Doria. - 1881. Pt. melanopogon (Schlegel) Peters var. papuanus Peters und Doria von Mansinam, N. W. Neu-Guinea. Ann. Mus. Civ. St. Nat. Genova XVI p. 690. - 1888. Pt. melanopogon von Port Moresby Jentink 1.c. p. 143. - 1897. Pt.papuanus Trouessart 1. c. p. 80. - 1897. Heller, Abh. Mus. Dresd. VI No. 8 p. 4.

Hab. Gebeh, Mansinam, Andei (N. W. Neu-Guinea), Loloki und Constantinhafen (S. 0. Neu-Guinea). Kubary.

[B. M.] $\sigma^{\pi} \sigma^{\pi}$ juv. Loloki, Finsch; 9 Gebeh, Bernstein; $\sigma^{\nearrow}$ Constantinhafen,

[Dresd. M.] ס’오 Andei, A. B. Meyer.

1c. Pt. degener Peters. - 1876. Pt. degener Peters von den Aru-InseIn. M. B. Akad. Berlin p. 318. - 1878. Dobson 1. c. p. 43. - 1897. Trouessart l. c. p. 80 . Hab. Aru-Inseln.

[B. M.] ठ Aru-Inseln, Cockerell.

11. Pt. neohibernicus Peters. - 1876. Pt. melanopogon Schlegel var. neohibernicus von Neu-Mecklenburg. M. B. Akad. Berlin 1876 p. 317-318. - 1878. Dobson l. c. p. 46, - 1897. Trouessart 1. c. p. 80 .

\section{Hab, Bismarck-Archipel.}

[B. M.] $\delta^{7}$ 우 Neu-Irland, S. M. S. Gazelle; 우 Neu-Lauenburg, Hübner; $15 \sigma^{\lambda} \sigma^{\pi} 13$ 우우 Neu-Pommern, Ralum, Finsch und Dahl, Kiniguan, Geisler.

[Dresd. Mus.] ठౌ Kiniguan, Gazellen-Halbinsel, Neu-Pommern, Geisler.

\section{Subgen. Pteropus Brisson s. str.}

1756. Brisson Quadrupeds p. 153. - 1870. Gray l. c. p. 102.

Schnauze lang; die Entfernung vom vorderen Augenwinkel zur Nasenspitze ist bedeutend grösser als die Breite der Schnauze an den Mundwinkeln. Unterschenkel nackt. Rücken behaart; wenn nur ein schmales Feld auf dem Rücken behaart ist, so sind die Ohren länger als die Mundspalte. Unterarm länger als $12 \mathrm{~cm}$. Der vorletzte obere Molar zeigt am hinteren Ende eine Ausbuchtung.

Typus: Pt. celaeno Hermann.

Hab. Madagaskar, Komoren, Aldabra-Inseln, Seychellen, Süd-Asien, Melanesien, Nord-Australien. Nicht vertreten u. a. auf den Maskaren, in Asien westlich von der indischen Wüste, nördlich vom Himalaya und nördlich von den Philippinen, in Polynesien mit Ausnahme von Samoa und Tonga und in Micronesien. Je eine Abart in jedem Gebiet.

Uebersicht der Arten:

Schwanzflughaut in der Mitte breiter als $10 \mathrm{~cm}$, von den Haaren nicht verdeckt .. . . . . . . celaeno 
Schwanzflughaut in der Mitte schmaler als $10 \mathrm{~cm}$, von den Haaren fast ganz oder ganz verdeckt:

Die Rückenbehaarung ist auf ein schmales Feld beschränkt, weil die Flughäute nahe der Rückenmitte ansetzen:

Unterrücken dunkler als der Nacken:

Kopf : sehr hell mit $\mathbf{T}$-förmiger Zeichnung . . . . .

Kopf gelbbraun ohne Zeichnung: Unterrücken heller als der Nacken:

Hinterrücken orangegelb

Hinterrücken weissgrau:

Brust dunkel

Brust hell .

Die Rückenbehaarung ist breit; die Flughäute setzen sich an die Körperseiten an:

Unterseite nicht schwarz:

Ohren viel länger als die Mundspalte:

Gesicht und Kehle schwärzlich . . . ... .

Gesicht und Kehle braun . Ohren ungefähr so lang wie die Mundspalte . . . . .

Unterseite schwarz, oft mit grauen Haaren sparsam gemischt:

Ohr bis $25 \mathrm{~mm}$ bei Alcoholexemplaren, bis $20,5 \mathrm{~mm}$ bei getrockneten Stücken; letztes Daumenglied ohne Kralle höchstens $40 \mathrm{~mm}$ lang . . . . . . . Ohr $26-28 \mathrm{~mm}$ bei Alcoholexemplaren, ungefähr $21 \mathrm{~mm}$ bei getrockneten Stücken; letztes Daumenglied ohne Kralle mindestens $42 \mathrm{~mm}$ lang . . . . .

Ohr länger als $29 \mathrm{~mm}$ resp. $22 \mathrm{~mm}$ : Ohr ca. $31 \mathrm{~mm}$ resp. $24-26 \mathrm{~mm}$ lang:

Färbung schwarz oder schwarz mit kastanienbraunem Nackenfleck, der nicht auf die Halsseiten übergreift . . .

Färbung schwarz mit gelbbraunem, auf die Halsseiten übergreifendem Nackenfleck :

\section{coronatus}

chrysoproctus

\section{grandis}

aruensis

keyensis

edwardsi und Verwandte medius und Verwandte

nicobaricus

tonganus

nicobaricus, modiglianii und aterrimus 


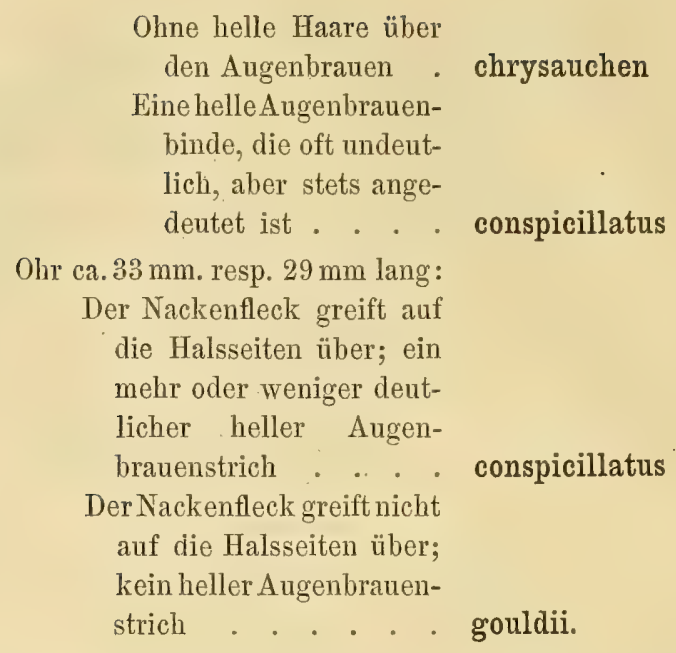

1. Pt. chrysoproctus Temminck. - 1837. Pt. chrysoproctus von Amboina, Temminck, Monogr. Mammal. II. p. 67, t. 35 f. 2 (Kopf). - 1844. Pt. argentatus Gray, Zool. Sulph. p. 30. - 1869. Pt. argentatus Fitzinger, l. c. p. 28. - 1869. Pt. chrysoproctus Fitz., l. c. p. 41-42. - 1878. Dobson, l. c. p. 47. - 1897. Trouessart, l. c. p. 80.

Abbildung des Schädels eines ơ. von Ceram auf Tafel [9] Fig. 5.

Hab. Amboina, Ceram, Arsiloeloe bei Ceram, Poeloe Pandjang bei Ceram, Goram, Koffian, Sanghir-Inseln. (Jentink 1888 l. c. p. 144.) (?) Matabello (Dobson, l.c. p. 48).

[B. M.] $\delta^{\nearrow}, 2$ \& Amboina, Beceari, S. Müller; $\sigma^{\nearrow}$ Ceram, Wallace; +9 Goram, Rosenberg.

[Dresd. Mus.] Ceram, Riedel.

2. Pt. aruensis (Schlegel) Peters. - 1867. Pt. melanopogon Schlegel var. aruensis Schleg. von den Aru-Inseln, M. B. Akad. Berlin p. 330. - (?) 1867. Pt. rubiginosus und fumigatus von Rosenberg, Reis naar de Zuidoostereilanden, s' Gravenhage p. 31. - 1878. Dobson, l. c. p. 45-46. - 1897. Trouessart, l. c. p. 80.

Abbildung des Schädels eines $\sigma^{\top}$ von den Aru-Inseln auf Tafel [6] Fig. 3.

Hab. Aru-Inseln (Wokam, Wonoembai) (Jentink 1888 l. c. p. 143).

[Berl. Mus.] $2 \sigma^{\top} \sigma^{\top}$ Aru-Inseln, Beccari, Bernstein.

[Dresd. Mus.] $\sigma^{\pi}$ und juv. Aru, Riedel.

3. Pt. keyensis (Schlegel) Peters. - 1867. Pt. melanopogon Schlegel var. keyensis Schleg. von den Key-Inseln, M. B. Akad. Berlin p. 330. - 1878. Dobson, l. c. p. 45-46. - 1896. Pt. chrysargyrus Heude von Toual, Petit Key, Mém. Hist. Nat. Empire Chinois III. p. 177 t. V f. 6 (Gebiss). - 1897. Trouessart, 1. c. p. 80.

Hab. Petit Key, Grand Key, Khoor (Jentink 1888 p. 143).

[Berl. Mus.] ठౌ, 2 ㅇ ㅇ Beccari, Rosenberg.

4. Pt. coronatus Thomas. - 1888. Pt. coronatus Thomas von Mioko bei NeuLauenburg, P. Z. S. p. 471, 484, t. XX. f. 2 (Kopf), t. XXI. f. 2. 3, Gebiss).

Hab. Mioko bei Neu-Lauenburg im Bismarck-Archipel. 
5. Pt. grandis Thomas. - 1887. Pt. grandis Thomas von Alu, ShortlandInseln, Ann. Mag. N. H. (5) XIX. p. 147, P. Z. S. p. 320-322, t. XXV. (Thier), Textfigur auf p. 321 (Gebiss). - 1888. von Rubiana, New Georgia, Thomas, P. Z. S. p. 470-471, 483. - 1897. Trouessart 1. c. p. 80.

Hab. Alu auf den Shortland-Inseln; Rubiana auf New Georgia (Salomon-Inseln). [Dres. Mus.] Alu, Ribbe.

6. Pt. celaeno Hermann. - 1758. Vespertio vampyrus L. partim Syst. Nat. Ed. X. 1 p. 31 Nr. 1. - 1804. Vespertio celaeno Hermann von Batavia, Observ. Zool. I, p. 13-15. - (?) V. nudus Hermann 1. c. p. 15-16. - 1810. Pt. edulis Geoffroy von Timor, Ann. Mus. XV. p. 90-91. - 1820. Pt. javanicus Desmarest von Java, Mammal. p. 109 Nr. 136. - 1837. Pt. funereus Temminck von Timor, Amboina, Borneo, Sumatra, Monogr. Mammal. II. p. $63-65$, t. 35 f. 4 (Kopf). - 1853. Pt. pluton 'Temminck von Bali und Lombok, Esqu. Zool. Côte Guiné I. p. 56-57. - 1878. Pt. edulis Dobs. 1. c. p. 49-51. - 1878. (?) Pt. pteronotus Dobson 1. c. p. 48 von Java. - 1881. Anderson, Cat. Mamm. Indian Mus. I. p. 100 partim. - 1887. Jentink l. c. p. 258-259. - 1888. Jentink 1. c. p. 144-146. - 1889. Jentink, Not. Leyd. Mus. p. 29 (Verbreitung). - 1891. Blanford, Fauna British India, Mamm. p. 259-260. - 1892. Pt. vampyrus Thomas, P.Z.S. p. $315,316$.

Abbildung des Schädels, wahrscheinlich nach einem der Original-Exemplare von Pt. edulis auf Tafel [1], Nr. 3 als Pt. edulis.

Hab. Hinterindien, südlich von Birma; Malakka, Sumatra, Java, Bali, Lombok, Timor, Banka, Borneo, Philippinen (Jentink 1889, Not. Leyd. Mus. p. 29), Natuna - Inseln (Thomas, Nov. Zool. I. 1894, p. 655, II. 1895, p. 489). Sipora, Mentawei-Inseln, Thomas, Ann. Mus. Civ. Genova Ser. 2, XIV. (XXXIV.) 1895, p. 5.

Wie Dobson's Pt. pteronotus sich von celaeno unterscheidet, vermag ich nicht zu erkennen, ohne das Exemplar gesehen zu haben.

[B. M.] $\sigma^{\top}$ ㅇ B Bangkok, von Martens; $3 \sigma^{\pi} 1$ \& Sumatra, von Petersen

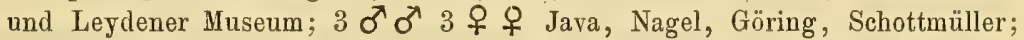
$\delta^{7}$ f B Borneo, Pagel und Marquis Doria; $\delta$ Indischer Archipel, Schweigger; $\sigma^{\nearrow}$ juv. Kupang auf Timor, von Martens; $2 \delta^{\nearrow} \delta^{\lambda}+f$ \& Luzon, Jagor; $3 \sigma^{\top} \sigma^{\top}$ Samar, Jagor.

[Dresd. Mus.] ठำ Java, von Schierbrand; Süd-0st Borneo, Wahnes; Mindanao, Schadenberg.

\%. Pt. edwardsi Geoffroy. - 1810. Pt. edwardsi Geoffr. partim von Madagaskar, Ann. Mus. XV. p. 92. - 1827. Pt. phaeops Temminck von Madagaskar, Monogr. Mammal. I. p. 178. - 1869. Fitzinger 1. c. p. $32-34 . \quad 1887$. Jentink 1. c. p. 259-260. - 1888. Jentink I. c. p. 146-147. - 1897. Trouessart 1. c. p. 81.

Abbildung des Schädels, wahrscheinlich nach einem der Original-Exemplare von Pt. phaeops Temm. aus Madagaskar auf Tafel [1], Nr. 4 als Pt. phaeops.

Hab. Madagaskar, Nossi-bé, Komoren (Jentink 1. c.).

[B. M.] $2 \sigma^{\nearrow} \sigma^{\nearrow}, 2$ 오오 Madagaskar, Leydener und Pariser Museum; $1 \delta^{\pi}, 2$ 우 우 Nossi-bé; $2 \delta^{\nearrow} \delta^{\nearrow}$ Angasilha, Komoren, von der Decken; ठㅇ․ Johanna, Komoren, Hildebrandt, Peters. 
\%a. Pt. seychellensis A. Milne-Edwards. - 1878. Pt. seychellensis A. Milne-Edwards von den Seychellen, Bull. Soc. Philom. p. 221-222. - 1868. Pt. edwardsi Peters, M. B. Akad. Berlin p. 368. - 1870. Perceval Wright, Spicilegia Biologica I. p. 15-16.

\section{Hab. Seychellen.}

Wir haben ein Exemplar von den Seychellen, welches die von Herrn A. MilneEdwards angegebenen Merkmale nicht besitzt und sich von Pt.edwardsi aus Madagaskar nicht unterscheidet. Da ich vermuthe, dass Pt. seychellensis nur ein Saisonkleid von Pt. edwardsi darstellt, so wage ich nicht, Pt. seychellensis als geographische Abart anzuerkennen.

[B. M.] $\sigma^{\top}$ Seychellen, Perceval Wright.

9b. Pt. aldabrensis True. - 1893. Pt. aldabrensis True von Aldabra, P. U.S. Nat. Mus. XVl. p. 533.

Eine Angabe der Unterschiede zwischen aldabrensis und edwardsi ist in der Originalbeschreibung nicht $z u$ finden. Soweit ich erkennen kann, unterscheidet sich aldabrensis nicht von Exemplaren, welche wir von Nossi-bé besitzen. Ich halte auch diese Species für edwardsi.

8. Pt. medius Temminck. - 1827. Pt. medius Temminck von Calcutta und Pondichery, Monogr. Mammal. I. p. 176-177. - (?) 1835. Pt. leucocephalus Hodgson von Nepal, Journ. Asiat. Soc. Bengal IV. p. 699-700. - 1869. Pt. medius und leucocephalus Fitzinger 1. c. p. 29-32. - (?) 1870. Pt. kelaarti Gray von Ceylon, Cat. Monk. Lem. Fruit-eating Bats. p. 104. - 1878. Dobson 1. c. p. 51-52. - 1881. Anderson, Cat. Mamm. Indian Mus. I. p. 101-102. - 1887. Pt. medius Jentink l. c. p. 259. - 1888. Jentink l. c. p. 146.

- 1891. Blanford, Faun. Brit. India, Mamm. p. 257-259. - 1897. Trouessart 1. c. p. 81 .

Abbildung des Schädels eines jüngeren $\widehat{\sigma}$ von Bengalen auf Tafel [9] Fig. 3.

Hab. Vorderindien bis Birma, Ceylon; nach Westen bis zum Indus.

Ob Pt. leucocephalus und kelaarti besondere geographische Abarten darstellen, vermag ich nicht zu entscheiden, weil mir genügendes Vergleichsmaterial fehlt. Unser Exemplar aus Ceylon zeigt genau die für kelaarti angegebenen Unterschiede.

[B. M.] $2 \sigma^{\nearrow} \sigma^{\top}, 2$ 우 우 Madras, Mitchell; 2 우우 Bengalen, Lamare-Piquot; ठ’ Ceylon, Redemann.

9. Pt. nicobaricus Fitzinger. - 1861. Pt. nicobaricus Fitzinger von Car-Nicobar, Zelebor, Sitzb. Wien. Akad. XLII. p. 389. - 1868. Reise Novara, I. Säugeth. p. 11-12. - 1869. Fitzinger 1. c. p. 26-28. - 1878. Dobson 1. c. p. 54-55. - 1887. Jentink 1. c. p. 259. - 1888. Jentink 1. c. p. 147. - 1891. Blanford, Fauna British India Mamm. p. $260-261$ - - 1897. Trouessart 1. c. p. 81 . 1863. Pt. melanotus Blyth von den Nicobaren, Cat. Mamm. Mus. Asiat. Soc. Beng. p. 20.

Hab. Andamanen, Nicobaren, Ross-Insel im Mergui-Archipel (Dobson 1. c. p. 56), Nias bei Sumatra (Jentink 1888 p. 147).

[B. M.] 2 우우 Andamanen, Dobson. 
Ohne Vergleichung eines grösseren Materials kann ich über die Artselbständigkeit von Pt. modiglianii Thos. nicht urtheilen. Ich stelle 2 in Berlin aufbewahrte Exemplare von den Cocos-Inseln, also weit südlich von Engano, hierher. Das $\delta$ ist schwarz mit einem graubraunen, nach unten kastanienbraunen Nackenfleck und sein Unterarm ist $135 \mathrm{~mm}$ lang; das 우 ist ganz schwarz und hat einen Unterarm von $145 \mathrm{~mm}$ Länge. Eines unserer Andamanen-Stücke, ein 오, hat ebenfalls einen Unterarm von $145 \mathrm{~mm}$ Länge. Dobson hatte es seiner Zeit als junges $q$ bezeichnet; ich kann diese Meinung deshalb nicht theilen, weil bei diesem Stück zwischen der Canine und den Molaren ein weiter Zwischenraum vorhanden ist.

9a.Pt. modiglianii Thomas. - 1894. Pt. modiglianii Thomas von Engano bei Sumatra, Ann. Mus. Civ. Genova ser. 2, XIV. (XXXIV.) p. 106-108. - 1897. Trouessart l. c. p. 81.

\section{Hab. Engano bei Sumatra und Cocos-Inseln.}

[B. M.] $\widetilde{c}$ + Cocos-Inseln südlich von Sumatra.

Die westlich von Sumatra gelegenen Inseln scheinen in zoogeographlscher Beziehung sehr interessant zu sein. Thomas erwähnt für Engano $P t$. modiglianii (s. o.) für Si-Oban auf Ost-Sipora, Mentawei-Inseln Pt. edulis (s. d.) und Jentink (s. o.) für Nias $P t$. nicobaricus. Diese drei Formen halte ich für so nahe verwandt, dass nicht zwei davon dieselbe Gegend bewohnen werden. Entweder muss man also annehmen, dass Nias zoogeographische Beziehungen zu den Nicobaren und Andamanen hat, dass die Mentawei-Inseln als losgetrenntes Stück von Sumatra zu betrachten sind und dass Engano faunistisch sehr von Sumatra und Java abweicht, oder man muss glauben, dass diese Pteropus-Arten zu gewissen Jahreszeiten ihre Heimath wechseln.

9b. Pt. aterrimus Temminck. - Pt. aterrimus Temminck von Bawean, Coup d'oeil sur les possessions néerlandaises dans l'Inde arquipélagique I. p. 333.

\section{Hab. Bawean nördilich von Java.}

[B. M.] \& Bawean, Diard.

Temminck hat [Esqu. Zool. 1853 p. 59] seinen Pt. aterrimus wieder eingezogen, weil er glaubte, dass die Celebes- und Bawean-Exemplare zu einer und derselben Art gehören. Das Berliner Museum besitzt eines der Diard'schen OriginalExemplare. Dasselbe ist dem $\widehat{\sigma}$ von den Cocos-Inseln sehr ähnlich; der Unterarm ist $152 \mathrm{~mm}$ lang, der Kopf $71 \mathrm{~mm}$, das $0 \mathrm{hr}$ an dem ausgestopften Thier $22 \mathrm{~mm}$, das letzte Daumenglied ohne Kralle $43 \mathrm{~mm}$. Die Nackenbinde ist hellkastanienbraun, der Kopf und die Unterseite schwarzbraun, grau überflogen, am Kinn und Unterleib schwach kastanienbraun getönt. Der Rücken ist schwarzbraun und hellgrau melirt. Der Schädel zeigt die Sutur zwischen Basioccipitale und Sphenoideum verwachsen, zwischen der Canine und dem 2. Praemolar im Oberkiefer ist ein Diastema von $4 \mathrm{~mm}$. Die Zahnreihe vom Vorderrand der mittleren Incisiven bis zum Hinterrande des letzten Molaren ist $32 \mathrm{~mm}$ lang. Basilarlänge: $64,5 \mathrm{~mm}$, grösste Breite: $37 \mathrm{~mm}$. Das Gebiss ist, wie häufig bei den Pteropus-Schädeln, krankhaft verändert. Die Maasse von $P t$. aterrimus weisen vielmehr auf eine Aehnlichkeit mit Pt. nicobaricus als auf eine solche mit dem Pt. alecto hin.

10. Pt. alecto Temminck. - 1837. Pt. alecto Temminck von Menado auf Celebes, Monogr. Mamm. II. p. 75-76. - 1890 von Makassar, Jentink, Weber's Zool. Ergebn. I. 1 p. 96 und 125. 
Hab. Celebes.

[B. M.] $\sigma^{\top}$ ㅇ Menado, von Faber; $\sigma^{\lambda}, 2$ 우 Segerie bei Mracassar, A. B. Meyer, ô Macassar, Beccari.

[Dresd. Mus.] đิ juv. Minahassa, von Faber; ơ pull. Gorontalo auf Celebes, Riedel.

[Sarasin Samml.] ơ Buol. Nord-Celebes, ơ Bouthein, Süd-Celebes, 우 pull. Sokoijo, Mataua-See, 우 mit Embryo IV. 94, Tomohon, Minnahassa, Gebr. Sarasin.

11. Pt. chrysauchen Peters. - 1862. Pt. chrysauchen Peters von Batjan, M. B. Akad. Berlin p. 576, Anmerkung. - 1870. Pt. mysolensis Gray von Mysol, Cat. Monk. etc. p. 105. - 1878. Pt. alecto partim Dobson l. c. p. $56-57$.

Abbildung des Schädels eines $q$ von Ternate auf Taf. [6], Fig. 1 als Pt. alecto.

Hab. Batjan, Morotay, Ternate, Obi, Ghebe, Toleno bei Tabello nordöstlich von Halmahera, Dorei, Nordwest-Neu-Guinea, Mysol (Jentink 1888 p. 147-148), Salvatti, West-Neu-Guinea (Ann. Mus. Civ. Genova 1881 p. 689), Insel Pinon, West-Neu-Guinea (Berl. Iluseum) also Halmahera-Gruppe, Mysol, Salawatti, Ghebe und die nordwestliche Halbinsel von Neu-Guinea.

[B. M.] 주 우 Morotay, Frank; 오 Ternate, Bernstein; 3 우 B Batjan, Frank und Verreaux; $\widehat{\jmath}$ Ghebe, Bernstein; $\widehat{0}$ Insel Pinon, S. M. S. Gazelle; ㅇ Dorei, Neu-Guinea, Marquis Doria. - ㅇ juv. Nordwest-Neu-Guinea von Faber. glaize.

[Dresd. Mus.] Insel Hum gegenüber Has, Nordwest-Neu-Guinea, La-

12. Pt. conspicillatus Gould. - 1849. Pt. conspicillatus Gould von der FitzroyInsel, Ost-Australien, P. Z. S. p. 109. - 1877. von der Rockingham Bay, Ost-Queensland und von der Yule-Insel, Südost-Neu-Guinea, Ramsay, P. Linn. Soc. N. S. Wales II. p. 7. - 1878. Dobson 1. c. p. $61-62$. - 1879. vom SüdCap und von der Contance-Insel, Südost-Neu-Guinea, Ramsay 1. c. III. p. 242. - 1879. von Port Moresby, Südost-Neu-Guinea, Ramsay 1. c. IV. p. 85. - 1881. Peters u. Doria, Ann. Mus. Civ. Genova XVI. p. 689. - 1892. Douglas Ogilby, Cat. Austral. Mamm. p. 80. - 1896. von den Kiriwana-Inseln, Thomas, Nov. Zool. III. p. 526. - 1897. Trouessart l. c. p. 82. - 1897. Thomas, Ann. Mus. Civ. Genova (2) XVIII. p. 608.

Abbildung des Schädels eines 우 von der Fitzroy-Insel auf Taf. [9] Fig. 4.

Hab. Südost- und Ost-Neu-Guinea, Yule, Contance, Fitzroy-Insel, Rockingham Bay, Kiriwana-Inseln, Woodlark-Inseln, Grange-Inseln (Thomas 1897).

[B. M] ㅇ Fitzroy-Insel, Macgillivray; $4 \sigma^{\top} \sigma^{\top}, 1$ 우 Yule-Insel, d'Albertis.

[Dresd. Mrus.] Bongu, Deutsch-Neu-Guinea, Wahnes; Woodlark-Inseln, Gerrard; Madang, Deutsch-Neu-Guinea, Geisler.

Pt. conspicillatus scheint zu gewissen Zeiten grosse Wanderungen zn unternehmen und auf diesen in die Wohngebiete von gouldii einzudringen, ebenso wie zu gewissen Jahreszeiten poliocephalus neben scapulatus in derselben Gegend gefunden wird.

13. Pt. gouldii Peters. - 1867. Pt. gouldii Peters von Rockhampton, NordostAustralien, M. B. Akad. Berlin p. 703-705. - 1863. Pt. funereus Gould (nec 
Temminck), von Port Essington, Mamm. of Australia III. t. 30. - 1878. Pt. gouldii Dobson 1. c. p. 60. - 1880. Ramsay, P. Linu. Soc. N. S. Wales IV. p. 85. - 1888. Collett, Zool. Jahrb. II. p. 843-844. - 1892. Douglas Ogilby, Cat. Austral. Mamm. p. 79. - 1897. Trouessart l. c. p. 82.

Abbildung des Schädels eines 우 von Rockhampton auf Tafel [7], Fig. 2.

Hab. Thursday-Insel in der Torres-Strasse, Cap York, Bet-Insel (Ramsay 1880), Quail-Insel, Howick's Gruppe, Gould-Insel, Percy-Insel (Dobson l. c. p. 61), Seaforth, Mackay, Rockhampton, Herbert River in Central-Quensland (Collett).

[B. M.] 꾸우 Thursday-Insel, Finsch; 2 우우 Rockhampton, Godeffroy; of Nord-Australien, Schomburgk.

[Dresd. Mus.] Rockhampton, Godeffroy.

14. Pt. tonganus Quoy u. Gaimard. - 1830. Pt. tonganus Quoy u. Gaimard von Tonga-Tabu, Voyage l'Astrolabe, Zool. I. p. 74-77, t. 8 (Thier und Schädel). - 1830. Pt. vanicorensis Quoy u. Gaimard von den Vanicoro-Inseln 1. c. p. 77-80, t. 9. - 1837. Pt. tonganus und vanicorensis Temminck, Monogr. Mamm. II. p. 78 -81. - 1867. Pt. geddiei (Macgillivray) Peters von den Neu-Hebriden, M. B. Akad. Berlin p. 326. - 1869. Pt. tonganus und vanicorensis Fitzinger, Sitzb. Akad. Wien I. Abth. p. 55-58. - 1870. Pt. flavicollis Gray und Pt. vitiensis Gray von den Fidschi-Inseln, Cat. Monk. etc. p. 107 und 109. - 1878. Pt. keraudreni Dobson (ohne Synonyme) 1. c. p. 63-65. 1880. Dobson, Rep. Brit. Assoc., Rep. on accessions to our knowledge of the Chiroptera during the past two years (1878-1880) p. 5. - 1897. Trouessart 1. c. p. $82-83$.

Abbildung des Schädels eines $\widehat{o}$ von Neu-Caledonien auf Tafel [7], Fig. 3 als Pt. geddiei.

Hab. Vanikoro-Inseln, Neu-Caledonien (Dobson, Report p. 5), Neu-Hebriden, Fidschi-Inseln, Wallis-, Freundschafts- und Samoa-Inseln.

[B. M.] $2 \sigma^{\nearrow} \sigma^{\lambda} \mathrm{Neu}$ - Caledonien, Verreaux; $2 \sigma^{\lambda} \sigma^{\lambda}$ juv. Tonga, Godeffroy;

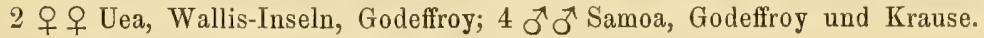

[Dresd. Mus.] Samoa-Inseln, Godeffroy.

\section{Subgen. Spectrum Gray.}

1870. Cat. Monk. Lem. Fruit-eating Bats p. 100-102.

Schnauze kurz oder ziemlich kurz; die Entfernung vom vorderen Augenwinkel zur Nasenspitze ist ungefähr so gross wie die Breite der Schnauze an den Mundwinkeln; Unterschenkel bald nackt, bald behaart; Rücken behaart; Unterarm länger als $10 \mathrm{~cm}$; wenn der Unterarm kürzer als $12 \mathrm{~cm}$. ist, so sind die Unterschenkel nackt. Der vorletzte obere Molar hat am hintern Ende keine Ausbuchtung und ist hinten höchstens so breit wie vorn.

Typus: Pt. vulgaris Geoffr.

Hab. Maskarenen, Madagaskar, Hinterindien, Ostindischer Archipel nach Norden bis zu den Riukiu-Inseln, Mikronesien, Melanesien und Samoa und Tonga. Nicht vertreten in Vorderindien und dem grösseren Theile von Polynesien. 
Uebersicht der Arten:

Unterschenkel dicht behaart bis zu dem Fussgelenk:

Ohr länger als $25 \mathrm{~mm}$ :

Ohr länger als $29 \mathrm{~mm}$. . . . . . poliocephalus p. 21

Ohr kürzer als $27 \mathrm{~mm}$. . . . . . . leucopterus p. 26

Ohr kürzer als $24 \mathrm{~mm}$ :

Rücken ungefähr ebenso gefärbt wie der Nacken

Rücken sehr verschieden vom Nacken in der Färbung:

Ohr sehr kurz, so lang wie der siebente Theil des Unterarmes . . . . . vetulus und vampyrus

Ohr länger, mindestens so lang wie

pselaphon p. 27 der sechste Theil des Unterarmes:

Oberschenkel heller als der

anetianus und rayneri

p. 22

Oberschenkel nicht heller als der Nacken . . . . . samoensis p. 22, dasymallus, loochoensis, formosus p. 27

Unterschenkel nicht bis zu dem Fussgelenk dicht behaart:

Unterschenkel zu zwei Dritteln dicht behart:

Aeusserer Ohrrand stark eingebuchtet

rodricensis p. 30

Aeusserer Ohrrand nicht oder sehr wenig eingebuchtet:

Ohr halb, so lang wie der Daumen

Ohr kürzer als die Hälfte der Länge des Daumens . . . . . . . . brunneus p. 22

Unterschenkel nackt:

Ohr länger als $22 \mathrm{~mm}$ bei trockenen, $30 \mathrm{~mm}$ bei Alcohol-Exemplaren:

Ohr ungefähr so lang wie die Entfernung vom vorderen Augenwinkel zur Nasenspitze:

Ohr schmal und zugespitzt. scapulatus p. 22

Ohr breit, oben abgerundet. livingstoni p. 30

Ohr viel länger als die Entfernung vom vorderen Augenwinkel zur Nasenspitze:

Schwanzflughaut unterhalb des Afters nicht ausgebildet

Schwanzflughaut unterhalb des Afters breiter als $10 \mathrm{~mm}$ macrotis und epularius p. 23 assamensis p. 26

Ohr kürzer als $22 \mathrm{~mm}$ bei trockenen, $30 \mathrm{~mm}$ bei Alcohol-Exemplaren . . . . . . hypomelanus und mariannus nebst verwandten Formen p. 23-26, 27-29. 
1. Pt. poliocephalus Temminck. - 1827. Pt. poliocephalus Temminck von NeuHolland, Monogr. Namm. I. p. $179-180$. - 1837. II. p. 66-67. - 1863. von Illawara, vom Manning, Clarence und von Bathurst, Gould, Mamm. Austr. III. t. 28 (Thier). - 1865. Krefft, Two Papers on the Vertebrata of the Lower Murray etc. p. 1. - 1867. Krefft, Austral. Vertebr. p. 4. - 1869. Fitzinger l. c. p. 38-40. - 1878. Dobson 1. c. p. 31-32. - 1888. Jentink 1. c. p. 253. - 1887. Collett, Zool. Jahrb. II. p. 842. - 1888. Jentink l. c. p. 139. 1892. Douglas Ogilby, Cat. Austral. Namm. p. 78. - 1897. Trouessart 1. c. p. 78. - 1897. A. F. de Seabra Jorn. Sc. Math. Phys. Nat. Lisboa V. 2 ser. p. 117-125 (erschienen 1898).

Abbildung des Schädels nach einem $\delta^{\lambda}$ von Sidney auf Taf. [7] Fig. 1.

Hab. Südost-Australien, Küste von Melbourne bis Mackay, Tasmanien, Illawara unweit des Hunter, Manning, Clarence, Bathurst (Gould), Cap Upstart, Port Stephens (Dobson), Sydney, Melbourne, Brisbane (Krefft), Seafort Mackay und Mittel-Queensland (Collett), Trobriand-Inseln (Dresd. Mus.); Timor (?) (de Seabra).

Von dieser Art weiss man sicher, dass sie wandert. Nach Krefft fehlt sie im unteren Murray - und Darling-Gebiet. Bei Sydney ist sie vom December bis Februar oder Januar zu finden. 1864 kamen diese Flederhunde bis Melbourne nach Süden. Jentink zähIt 2 Stücke von Tasmanien auf, Collett erwähnt sie für Mackay aus dem Juli. Im Leydener Catalog sind 2 Exemplare von Cap York aufgeführt, F. de Seabra nennt diese Art für Timor, daneben allerdings auch samoensis und pselaphon. Eine Nachprüfung der von ihm zı 10 Species gerechneten Exemplare dürfte sich dringend empfehlen.

[B. M.] $ð$ 우 Sydney, Krefft; $2 \circlearrowleft ð$ Neu-Süd-Wales, Wessel und Frank. [Dresd. M.] \& Victoria, Krone; ㅇ Müller; ot Trobriand-Inseln, Meek.

2. Pt. vetulus Jouan. - 1863. Pt. vetulus Jouan von Neu-Caledonien, Mém. Soc. Sc. Cherbourg p. 90. - 1870. Pt. ornatus Gray von Port de France, Neu-Caledonien, Cat. Monk. Lem. Fruit-eating Bats. p. 105-106. 1878. Dobson 1. c. p. 27-28. - (?) 1878. Pt. germaini Dobson von NeuCaledonien, P. Z. S. p. 875 und 1880 Rep. Brit. Ass. 1880 p. 4-5. 1887. Jentink l. c. p. 253 . - 1888. Jentink I. c. p. 189. - 1897. Trouessart l. c. p. 78 . Fig. 2.

Abbildung des Schädels nach einem jüngeren $\&$ von Neu-Caledonien auf Tafel [1]

\section{Hab. Neu-Caledonien.}

Unsere Exemplare von vetulus sind in der Färbung sehr verschieden und zwar so, dass man wenigstens bei unserem Material Geschlechtsunterschiede in der Färbung nicht feststellen kann. Ein junges $\widehat{\sigma}$ hat ungefähr die Färbung von germaini Dobs. und deshalb glaube ich, besonders weil die von Dobson angegebene Kürze des Unterarmes auf ein junges Thier hindeutet, dass germaini mit vetulus zusammenfällt. Ob die Form von den Loyality-Inseln, welche Dobson erwähnt, von vetulus verschieden ist, vermag ich olne Untersuchung des betreffenden Exemplars nicht $\mathrm{zu}$ beurtheilen.

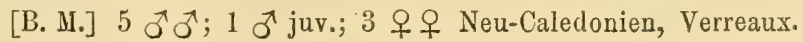


3. Pt. anetianus Gray. - 1870. Spectrum anetianum Gray von Aneiteum, Neu-Hebriden, Cat. Monk. Lem. Fruit-eating Bats p. 101-102. - 1878. Pt. aneiteanus Dobson 1. c. p. 29 Taf. IV. Fig. 2 (Gebiss). - 1887. Jentink 1. c. p. 253. - 1888. Jentink 1. c. p. 139. - 1897. Pt. aneitanis Trouessart 1. c. p. 78.

Hab. Aneiteum (Annatom), Neu-Hebriden.

4. Pt. Samoensis Peale. - 1848. Pt. samoensis Peale von Tutuilla, Manua, Upolu und Savai, Samoa-Gruppe, Expl. Exp. Vincennes, Quadrupeds p. 20 1. ed., Atlas, Mamm. Taf. II. (Thier). - 1858. Pt. samoensis Cassin, U. S. Expl. Exp. Mamm. p. 7-10, Abbildung des Schädels p. 10. - 1869. Pt. samoensis Fitzinger 1. c. p. 60-61. - 1870. Pt. nawaiensis Gray von Nawai, Fidschi-Inseln, Cat. Monk. etc. p. 107. - 1870. Pt. vitiensis Gray. von Ovalau, Fidschi-Inseln 1. c. p. 109. - 1874. Pt. whitmei Alston von den SamoaInseln, P. Z. S. p. 96 Taf. XIV (Thier). - 1887. Jentink 1. c. p. 253. 1888. Jentink l. c. p. 140. - 1897. Trouessart 1. c. p. 79. - 1897. A. F. de Seabra. Jorn. Lisboa p. 117-125.

Abbildung des Schädels eines $0^{7}$ von Samoa auf Tafel [3] Fig. 1.

Hab. Samoa-Inseln, Fidschi-Inseln (Ovalau, Nawai, Witi Lewu).

[B. M.] ơ Samoa, Godeffroy; \& Upolu, Samoa, Godeffroy; ơ Witi Lewu, Fidschi-Inseln, Godeffroy; ठ̛ Ovalau, Fidschi-Inseln.

5. Pt. layneri Gray. - 1870. Pt. rayneri Gray von Guadalcanar und San Christoval, Salomons-Inseln, Cat. Monk. Lem. Fruit-eating Bats p. 108. 1878. Dobson 1. c. p. 33-34. - 1887. Thomas, P. Z. S. p. $322 .-1888$. Thomas, P. Z. S. p. 472, 483. - 1897. Trouessart I. c. p. 78.

Hab. Südliche Salomons-Inseln (San Christoval, Guadalcanar).

[Dresd. Mius.] Aola, Guadalcanar, Woodford.

6. Pt. brunneus Dobson. - 1878. Pt. brunneus Dobson von der Percy-Insel, Ostküste von Australien, Cat. Chiropt. p. 37-38, Taf. III. Fig. 4 (Ohr). 1892. Douglas Ogilby, Cat. Austr. Mamm. p. 79. - 1897. Trouessart 1. c. p. 79. 1897. A. F. de Seabra 1. c. p. 117-125.

Hab. Percy-Insel, Ostküste von Australien.

\%. Pt. scapulatus Peters. - 1862. Pt. scapulatus Peters von Cap York, Nordaustralien, M. B. Akad. Berlin p. 574-576. - 1866. Pt. elseyii Gray von der Claremont-Insel und von Nordost-Australien, Cat. Monk. etc. p. 108. 1878. Pt. scapulatus Dobson 1. c. p. $41-42$, Taf. IV. Fig. 3 (Gebiss). 1887. Collett, Zool. Jahrb. II. p. 843. - 1887. Jentink 1. c. p. 254. - 1888. Jentink 1. c. p. 141. - 1892. Douglas Ogilby, Cat. Austral. Mamm. p. 80. 1897. Trouessart 1. c. p. 79. - 1897. Collett, P. Z. S. p. 318-319.

Abbildung des Schädels eines $q^{\text {? }}$ von Cap York auf Tafel [7] Nr. 4.

Hab. Nord- und Nordost-Australien, Daly-Fluss (Arnhem-Iand) [Collett VIII. 1897], Cap York, Claremont-Insel, Mackay, Rockhampton [Collett 1887].

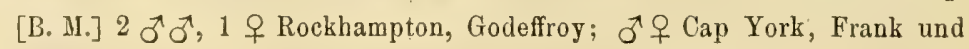
Salmin. 
8. Pt. macrotis Peters. - 1867. Pt. macrotis Peters von Wokam, Aru-Inseln (fälschlich für Boeroe von Peters angegeben) M. B. Akad. Berlin p. 327-329. - 1867. Pt. insignis von Rosenberg von Wokam, Reis Zuidoostereilanden p. 31. Abbildung des Schädels nach dem Leidener Exemplare von Wokam auf Tafel [6] Fig. 2.

Hab. Aru-Inseln (Wokam).

[Dresd. Mus.] Aru-Inseln, Ribbe und Kühn.

9. Pt. epularius Ramsay. - 1877. Pt. (Epomops?) epularius Ramsay von Katow, Neu-Guinea, P. Linn. Soc. New South Wales II. p. 8. - 1878. Pt. macrotis Dobson von der Yule-Insel, l. c. p. 43, Taf. III. Fig. 5 (Ohr).

Aus der von Dobson gegebenen Beschreibung des Exemplares von der YuleInsel habe ich nicht die Ueberzeugung gewinnen können, dass die Stücke von den Aru-Inseln und der Yule-Insel übereinstimmen. Der Dresdener Pt. macrotis entspricht vollständig der von Peters gegebenen Originalbeschreibung, während das Thier von der Yule-Insel ebenso wie Ramsay's epularius von Katow sich durch andere Maasse erbeblich unterscheiden. Ich empfehle vorläufig beide Species auseinanderzustellen.

Hab. Neu-Guinea südlich vom centralen Hochgebirge, Katow, Yule-Insel.

Die nun folgenden Formen sind sich sehr ähnlich, und ich halte es wohl für möglich, dass Pt. hypomelanus Temm., griseus Cuv., pallidus Temm., tomesii Ptrs., ocularis Ptrs., condorensis Ptrs., fuscus Dobs., lombocensis Dobs. und natalis Thos. zu einer und derselben Art gehören und nicht einmal als geographische Abarten getrennt werden können.

Wenn auf den Banda-Inseln, wie Jentink 1. c. 1888 p. 141 und 149 angiebt, Pt. griseus neben hypomelanus vorkommt, so stellen beide entweder verschiedene Kleider einer und derselben Form dar oder aber hypomelanus ist dort nur zu bestimmten Jahreszeiten zu finden, oder, wie z. B. beim Wald- und Feldhasen, wir haben es hier mit solchen Formen zu thun, welche durch etwas verschiedene Lebensweise gewisse unterscheidende Merkmale gewonnen haben. Eine endgiltige Lösung dieser Fragen ist erst möglich, wenn wir mehr über die Wanderzüge der Flughunde und die Veränderungen in der Färbung und Gestalt während der verschiedenen Jahreszeiten erfahren haben.

Die individuellen Abänderungen bei hypomelanus nach Färbung, Grösse und Gebiss sind, wie mir das Studium einer grösseren Anzahl von Talaut-Exemplaren des Dresdener Museums gezeigt hat, so erstaunlich mannigfach und vom Gesehlecht scheinbar unabhängig, dass nur die Bearbeitung eines sehr grossen Materials, welches von demselben Fundorte aus jedem Monate Exemplare verschiedener Geschlechter und verschiedenen Alters umfasst, hier Rettung schaffen kann.

10a. Pt. lypomelanus Temminck. - 1853. Pt. hypomelanus Temminck von Ternate, Esqu. Zool. Côte Guiné I. p. 61-62. - 1867. Fitzinger 1. c. p. 50 -52. - 1870. Pt. tricolor Gray von Ternate, Cat. Monk. Lem. Fruit-eating Bats p. 108. - 1878. Pt. hypomelanus Dobson 1. c. p. 57-58. - 1880 von Cam bodja Dobson, Rep. Brit. Ass. 1880 p. 5. - 1883. Jentink, Not. Leyd. Mus. V. p. 173. - 1887. Jentink 1. c. p. 261-262. - 1887. Thomas, P. Z. S. p. 322. - 1888. Jentink l. c. p. 148-149. - 1890. Jentink, Weber's Zool. Erg. I. 1 p. 96 und 125. - 1893. Everett, P. Z. S. p. 494. - 1894. Thomas, 
Nov. Zool. I. p. 655. - 1894. Thomas, Ann. Mus. Civ. Genova ser. 2, vol. XIV. p. 106. - 1895. de Elera, Cat. Syst. p. 5. - 1895. Thomas, Nov. Zool. II. p. 163 und 489. - 1895. Thomas, Ann. Mus. Civ. Genova ser. 2 a, vol. XIV. p. 664. - 1896. Thomas, Nov. Zool. III. p. 526. - 1896. Elliot, Field Columb. Mus. II. Zool. Ser. vol. I. Nr. 3 p. $76-77$. - 1897. Trouessart 1. c. p. 82.

Hab. Nördliche Salomons-Inseln (Alu, Shortland-Inseln Thomas 1887), Kiriwana-Inseln, Trobriand-Gruppe (Thomas 1896), Fergusson-Inseln, EntrecasteauxGruppe (Thomas 1895 p. 163), Neu-Guinea, nördlich vom centralen Hochgebirge (Huon-Golf, Dobson p. 58, Mafor, Berl. Mus.), Banda-Inseln (Jentink 1888), Ternate (Temminck), Morotai, Halmahera, Kaioa-Inseln bei Batjan (Jentink 1888), Sulu-Inseln (Soela Bessie, Jentink 1884), Marangas-Inseln, Dresd. Mus.), Talaut-Inseln (Dresd. Mus.), Sanghir-Inseln (Dobson 1878), Nord - und SüdCelebes (Jentink 1890), Philippinen (Elliot 1896), Borneo (Everett 1893), NatunaInseln (Thomas 1894 nnd 1895), Cambodja (Dobson 1880), Engano bei Sumatra (Thomas 1894 p. 106), Mentawei-Inseln bei Sumatra (Thomas p. 664).

$\mathrm{Zu}$ Pt. hypomelanus im engeren Sinne d. h. zu der von Ternate zuerst bekannt gewordenen Form ziehe ich folgende Stücke des Berliner und Dresdener Museums:

[B. M.] 4 우 Sanghir-Inseln, Platen, A. B. Meyer, Marquis Doria;

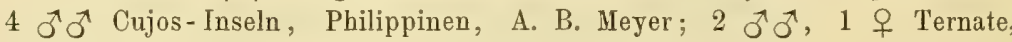
A. B. Meyer und Bernstein; 3 ō $\widehat{0}, 1$ o Celebes - Gorontalo, Macassar, A. B. Meyer, Beccari; ô Mafur, N.W.-Neu-Guinea, Beccari.

[Dresd. Mus.] ơ Mindanao, Semper; 8 Stück Siao, A. B. Meyer; GrossSanghir, A. B. Meyer; 29 Stück Talaut-Inseln - Kabruang, Esang, Karkellang; ơ Marangas, Sulu-Inseln, Hose. - Woodlark-Inseln, Meek.

[Sarasin'sche Samml.] ơㅇ Macassar, Sarasin.

Mit Pt. hypomelanus zu vergleichen sind:

10b. Pt. griseus E. Geoffroy St. Hilaire. - 1810. Pt. griseus Geoffroy von Timor, Ann. Mus. XV.p. 94 Taf. 6 (Thier). - 1869. Fitzinger l. c. p. 61-63.

Hab. Timor.

10c. Pt. pallidus Temminck. - 1827. Pt. pallidus Temminck von Banda, Monogr. Mamm. I. p. 184-186, Taf. XV. Fig. 8 und 9 (Schädel). - 1867. Fitzinger 1. c. p. $52-53$.

Abbildung des Schädels nach einem jüngeren $q$ von Banda auf Tafel [1] Fig. 5 als Pt. griseus.

\section{Hab. Banda-Inseln.}

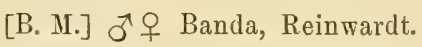

Peters hat diese beiden Arten (IJ. B. Akad. Berlin 1867 p. 326) „nach directer Vergleichung von Originalexemplaren und Vergleichung der Beschreibungen" für übereinstimmend erklärt und Dobson (1. c. p. 44) hat dies ebenso wie Trouessart (1. c. p. 80) angenommen. - Auf den Banda-Inseln lebt nach Jentink (1888 p. 149) neben griseus auch hypomelanus.

10 1. Pt. ocularis Peters. - 1867. Pt. ocularis Peters von Ceram, M. B. Akad. Berlin p. 326-327. - 1878. Dobson l. c. p. 62. - 1888. Thomas, P. Z. S. p. 471, Anmerkung. - 1897. Trouessart 1. c. p. 82.

Abbildung des Schädels eines alten $\widehat{\sigma}$ von Ceram auf Tafel [4] Fig. 5 und 6.

Hab. Ceram. 
Dobson (I. c. p. 57) führt Pt. alecto für Ceram auf aus den Sammlungen von Wallace. Pt. ocularis hat mit der alecto-Form von Ternate eine gewisse äussere Aehnlichkeit, weil beide schwarze Unterseite haben. Ich glaube nicht, dass 2 Formen der Untergattung Pteropus auf Ceram neben einander leben werden. Pt. chrysoproctus ist von Ceram allgemein bekannt, dagegen besitzen weder das Berliner noch das Leydener Museum eine alecto-ähnliche Form von der AmboinaGruppe. Es ist deshalb sehr interessant, wenn nachgewiesen wird, dass das Exemplar g von Pt. alecto des Britischen Museums wirklich zu Pt. alecto gehört. Denn in diesem Falle sind zwei Möglichkeiten vorhanden: entweder wandert alecto resp. chrysauchen zu Zeiten nach Süden, oder aber die chrysoproctus-Gruppe (chrysoproctus, aruensis, keyensis, coronatus, grandis) ist als gleichwerthige Untergattung neben die alecto-Gruppe zu stellen. Der erste Fall, ein Ziehen aus einer Zone in die andere, ist bisher für alecto-ähnliche Formen noch nicht nachgewiesen; der zweite Fall ist vorläufig noch sehr unwahrscheinlich, da aus dem gesammten von der chrysoproctus-Gruppe bewohnten Gebiete sonst nirgendwoher das Auftreten einer alecto-Form bisher bekannt ist und beide Gruppen ausserdem sehr nahe mit einander verwandt sind und im Schädelbau nur Grössenverhältnisse Verschiedenheiten zwischen beiden charakterisiren.

[B. M. J ơ Ceram, Wallace.

10 e. Pt. tomesii Peters. - 1858. Pt. hypomelanus (Temminck) Tomes von Labuan bei Borneo, P. Z. S. p. 536. - 1868. Pt. hypomelanus Temminck, var. Tomesii Peters von Sarawak, Borneo, M. B. Akad. Berlin p. 626.

Hab. Borneo.

10f. Pt. vociferus Peale. - 1848. Pt. vociferus Peale von Mangsi in der Strasse von Balabak, U. S. Explor. Exp. 1 ed. p. 19. - 1858. Pt. mackloti Cassin (nec. Temm.) 1. c. 2 ed. p. 10.

Hab. Mangsi in der Strasse von Balabak (vergl. p. 11).

10 g. Pt. condorensis Peters. - 1869. Pt. condorensis Peters von Pulo Condor, M. B. Akad. Berlin p. 393. - 1878. Pt. nicobaricus Dobson 1. c. p. 55.

Abbildung des Schädels eines $\sigma^{\pi}$ von Pulo Condor auf Tafel [8] Fig. 2.

Hab. Pulo Condor, südlich von Nieder-Cochinchina.

[B. M.] of Pulo Condor, Pariser Museum.

Dass diese Species mit $P t$. nicobaricus nichts zu thun hat, beweist die Vergleichung des auf unserer Tafel 8, Fig. 2 abgebildeten Schädels mit einem Schädel von nicobaricus. Der vorletzte obere Molar ist nach hinten verschmälert, also gehört Pt. condorensis zur Untergattung Spectrum. Auf der Abbildung erscheint der linke vorletzte obere Molar hinten ebenso breit wie vorn; der Zeichner hat nicht genügend dargestellt, dass dieser Zahn schräg nach innen und unten sehr stark abgekaut ist, und dadurch die hintere Hälfte des Zahnes zu breit gezeichnet.

10 h. Pt. fuscus Dobson. - 1878. Pt. fuscus Dobson von Celebes, 1. c. p. 59-60, Taf. IV. Fig. 5 (Gebiss).

\section{Hab. Celebes.}

Ich führe diese Species noch gesondert auf, obwohl ich, ohne das OriginalExemplar gesehen zu haben, ziemlich sicher bin, dass fuscus als Synonym zu hypomelanus zu ziehen ist. Die Gestalt des letzten, oberen Molaren ist für die 
Artberechtigung nicht beweiskräftig, da dieser Zahn durch Abkauung sehr verschiedene Gestalten annimmt. Im Berliner Museum befinden sich u. a. von Siam 3 Schädel der grossohrigen hypomelanus-Form, welche ich vorläufig mit assamensis bezeichne. Von diesen hat einer einen ähnlichen oberen letzten Molar wie fuscus.

10 i. Pt. macassaricus Heude. - 1896. Pt. macassaricus Heude von Macassar, Mém. Hist. Nat. Emp. Chinois III. p. 177 Taf. V. Fig. 4 (Gebiss).

Hab. Macassar, Celebes.

Dass diese Species unter die Synonyme von hypomelanus gehört, ergiebt sich aus der folgenden Beschreibung mit genügender Sicherheit.

"La tête est blonde, le cou et le haut du dos roux vif, le reste du dos est brun tiqueté de gris. Le dessous est roux à peu-près uniforme. Un sujet sur dix me montre le dos d'un roux foncé ainsi que la poitrine et le ventre. Le crâne est plus effilé et les dents sont plus comprimées latéralement que chez le tricolor [N.B. Heudes tricolor, welcher mit temmincki verwandt ist]; mais le crâne est bien plus petit que celui du medius de Saïgon, alorsque les molaires y sont plus grosses et plus rapprochées."

10 k. Pt. lombocensis Dobson. - 1870. Pt. mackloti var.? von Lombock, Gray, Cat. Monk. Lem. Fruit-eating Bats. p. 110. - Pt. lombocensis Dobson von Lombock, l. c. p. 34-35, Taf. III. Fig. 4 (Ohr), Taf. IV. Fig. 4 (Gebiss). 1887. Thomas, P. Z. S. p. 512-513.- - 1897. Trouessart, l. c. p. 79.

\section{Hab. Lombock.} bildungen).

Pt. lombocensis ist Pt. griseus im Gebiss sehr ähnlich (cf. die citirten Ab-

101. Pt. natalis Thomas. - 1887. Pt. natalis Thomas von der Christmas-Insel, südlich von Sumatra, P. Z. S. 1887 p. 511-513, Taf. XLI. (Thier). - 1888. Thomas, P. Z. S. p. 532. - 1897. Trouessart 1. c. p. 81.

Hab. Christmas-Insel, südlich von Sumatra.

11. Pt. assamensis Mc Clelland. - 1839. Pt. assamensis Mc Clelland von Assam, P. Z. S. p. $148-149$.

Abbildung des Schädels eines ${ }^{7}$ von Bangkok auf Tafel [4], Fig. 8.

Hab. Assam, Bangkok und Siam.

Unter Vorbehalt stelle ich 4 Exemplare des Berliner Museums, 3 우우, welche von Verreaux gekauft worden sind und aus Siam stammen sollen, sowie ein $\widehat{\jmath}$, welches von Martens in Bangkok sammelte, zu Pt. assamensis Mc Clelland. Die drei 우우 haben einen schwarzbraunen Leib, das $\delta$ entspricht sehr gut der OriginalBeschreibung. Charakteristisch ist für diese Thiere der breite, kurze Kopf, die langen Ohren und die verhältnissmässig breite Schwanzflughaut unterhalb des Afters.

[B. M.] 3 우 Siam, Verreaux; đ Bangkok, von Martens.

12. Pt. leucopterus Temminck. - 1853. Pt. leucopterus Temminck von unbestimmter Herkunft, Esq. Zool. Côte Guiné I. p. 60-61. - 1867. Peters, M. B. Akad. Berlin p. 323-324. - 1870. Pt. chinensis Gray von China (?), Cat. Monk. Lem. etc, p. 111. - 1870. Spectrum leucopterum Gray von den Phi- 
lippinen (?), 1. c. p. 102. -- 1878. Dobson, 1. c. p. 32-30, Taf. IV. Fig. 1, 1 a und $1 \mathrm{~b}$ (Schädel und Gebiss). — 1895. de Elera, Cat. Syst. p. 5. — 1897. Trouessart, l. c. p. 78.

Hab. China (?), Philippinen (?).

13. Pt. formosus Sclater. - 1873. Pt. formosus Sclater von Formosa, P. Z. S. p. 193, Taf. XXII. (Thiei). - 1878. Dobson, l. c. p. 26. - 1891. Sclater, P. Z. S. p. 677. - 1897. Trouessart, 1. c. p. 78.

Hab. Formosa.

14. Pt. loochoensis Gray. - 1870. Pt: loochoensis Gray von den Loochoo-Inseln, Cat. Monk. Lem. Fruit-eating Bats. p. 106. - 1878. Dobson, 1. c. p. 65. 1897. Trouessart, l. c. p. 83.

Hab. Riukiu-Inseln.

15. Pt. dasymallus Temminck. - 1827. Pt. dasymallus Temminck von Japan (Nagasaki und Yedo), Monogr. Mamm. I. p. 180-181, Taf. X. (Thier), Taf. XV. Fig. 10-11 (Schädel). - 1837. Temminck, l. c. II. p. 73-74. - 1850. Temminck, Fauna Japonica p. 12. - 1869. Fitzinger, l.c. p. 44-46. - 1878. Dobson, l. c. p. 25, Taf. III. Fig. 1 (Ohr). - 1887. Jentink, l. c. p. 252. 1888. Jentink, l. c. p. 138. - 1892. Ehmann, Mitth. Ges. Naturk. Ostasiens V. (47) p. 389. - 1897. Trouessart, 1. c. p. 77.

Abbildung des Schädels wahrscheinlich nach einem Leidener Exemplar auf Tafel [1], Fig. 1 .

Hab. Nagasaki und Yedo nach Temminck 1827. - Satsuma auf Kyushiu und Yakunoshima Temminck 1850 - auf die Riukiu-Inseln beschränkt, während er auf Kyushiu und Yakunoshima fehlt. Ehmann 1892.

16. Pt. pselaphon Lay. - 1829. Pt. pselaphon Lay von den Bonin-Inseln, 'Lool. journ. vol. 4 p. 457. - 1837. Pt. ursinus Kittlitz von den Bonin-Inseln, Temminck, Monogr. Mamm. II. p. 73. - 1837. Pt. pselaphon Temminck, l. c. p. 70-73, Taf. 37 (Thier), Taf. 36 Fig. 9, 10, 11 (Schädel). - 1839. Richardson, Zool. Beechey p. 11 Taf. II. (Thier), Collie 1. c. Anmerkung, Lebensweise und Anatomie. - 1869. Fitzinger, 1. c. p. 46-47. - 1878. Dobson, 1. c. p. 26-27. - 1887. Jentink, l. c. p. 253. - 1888. Jentink, 1. c. p. 139. - 1897. Trouessart, 1. c. p. 78.

Abbildung des Schädels, wahrscheinlich nach einem Leidener Exemplar auf Taf. [9], Fig. 1 als Pt. ursinus.

Hab. Bonin-Inseln, südöstlich von Japan.

[B. M.] $ぇ, 3$ 우 Bonin-Inseln, Rex u. Co., Brauns.

1\%. Pt. mariannus Desmarest. - 1822. Pt. mariannus Desmarest von den Mariannen, Mammal. Suppl. p. 547, Anmerkung 1. - 1824. Pt. keraudren Quoy et Gaymard von den Mariannen, Guam, Voyage Uranie Physic. Hist. Nat. Zool. p. 51-53, Atlas, Taf. 3 (Thier und Schädel). - (?) 1827. Pt. dussumieri Is. Geoffroy St. Hilaire, angeblich von Ostindien und Amboina, Dict. class. XIV. p. 71. - 1869. Fitzinger, 1. c. p. 53-55. - (?) 1878. Pt. insularis Dobson (nec Hombr. Jacq.) von Yap, Palau-Inseln, l, c. p. 65. 
Abbildung des Schädels eines $ᄋ$ von Guam auf Tafel [5], Fig. 15-16. Insel.

Hab. Guam, Mariannen und vielleicht Yap, Palau-Inseln und Hackenzie-

Möglicherweise ist der Flederhund der Palau-Gruppe verschieden von demjenigen der Mariannen; man müsste feststellen, ob nicht dussumieri Is. Geoffr. auf den Palau-Flederhund zu beziehen ist.

[B. M.] $2 \sigma^{\Uparrow} \sigma^{\nearrow}$ Palau-Inseln, Godeffroy; 우 Guam Kittlitz.

18. Pt. insularis Hombron u. Jacquinot. - 1842. Pt. insularis Hombron u. Jacquinot von den Karolinen, Voyage Pôle Sud Mamm. Taf. 5. - 1853. l. c. vol. III. p. 24. - 1869. Peters, M. B. Akad. Berlin p. 391-392. - 1883. von Ruck (Hegoleu), Peters, Sitzb. Ges. naturf. Fr. Berlin p. 2. - (?) 1890. Pt. lanigera H. Allen, angeblich von den Samoa-Inseln, P. Am. Phil. Soc. XXVIII. Nr. 132 p. $70-72$.

Abbildung des Schädels eines ơ und eines 오 von Ruck auf Taf. [5], Fig. 6-10() und $11-12\left(\sigma^{\top}\right)$.

Hab. Ruck, Hegoleu, Karolinen.

[B. MI.] 1 ðี, 2 우우 Ruck, Hegoleu, Finscb.

Ob Pt. lanigera von insularis verschieden ist, kann ich vorläufig nicht entscheiden; jedenfalls ist lanigera nicht von Samoa, da dort eine verwandte Art aus derselben Gruppe lebt, nämlich samoensis.

19. Pt. phaeocephalus Thomas. - 1882. Pt. phaeocephalus Thomas von der Mortlock-Insel, Karolinen, P. Z. S. p. 756 Taf. LIV. (Thier und Gebiss). - 1897. Trouessart, l. c. p. 83.

Als phaeocephalus (?) habe ich auf Tafel [5], Fig. 1-5 die Abbildung eines Schädels von Ponape, Karolinen gegeben, der von ualanus und insularis sich unterscheidet. $P t$. phaeocephalus könnte übrigens das 우 von insularis sein.

Hab. Mortlock-Insel, Karolinen.

20. Pt. ualanus Peters. - 1883. Pt. ualanus Peters von Kuschai, Karolinen, Sitzb. Ges. naturf. Fr. Berlin p. 1.

Abbildung des Schädels nach einem ơ von Kuschai auf Tafel [5], Fig. 17-22 und als phaeocephalus (?) denjenigen eines Exemplars von Ponape, welcher sich von Schädeln des ualanus nicht unwesentlich unterscheidet.

Hab. Kuschai (Ualan), (?) Ponape, Karolinen.

[B. M.] 5 ठ઼ઠ઼, 4 우우 Kuschai, Finsch.

21. Pt. admilalitatum Thomas. - 1894. Pt. admiralitatum Thomas von den Admiralitäts-Inseln, Ann. Mag. Nat. Hist. ser. 6 vol. XIII. p. 293. - 1897. Trouessart, l. c. p. 82.

Abbildung des Schädels nach einem ठ઼ von Neu-Pommern auf Taf. [3], Fig. 2.

Hab. Admiralitäts-Inseln, Neu-Pommern (Ralum), Matupi bei Neu-Pommern.

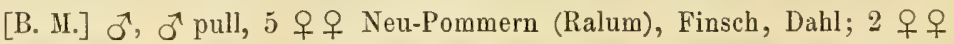
Matupi, Dahl.

Nach der von Thomas gegebenen Beschreibung scheinen die Exemplare von Neu-Pommern zu dieser Art zu gehören; eine Vergleichuug der Abbildung des Schädels mit den Schädeln der Original-Exemplare wird vielleicht die Frage aufklären. 
22. Pt. tuberculatus Peters. - 1869. Pt. tuberculatus Peters von unbekannter Herkunft, M. B. Akad. Berlin p. 393-394. - 1878. Dobson, p. 58-59. 1897. Trouessart, l. c. p. 82.

Abbildung des Schädels nach dem Pariser Original-Exemplar auf Taf. [8], Fig. 3.

Hab. Unbek annt.

Nach der Abbildung ist es unmöglich, Peter's Ansicht beizustimmen, dass tuberculatus mit macklotii, celebensis und jubatus verwandt ist; Dobson hat denn auch tuberculatus nicht zu den obigen Arten, sondern zwischen hypomelanus und fuscus gestellt.

Meiner Ansicht nach erinnert die Gestalt des Schädels und der Zähne von tuberculatus noch am meisten an pselaphon. Peters giebt an, dass die Behaarung ähnlich wie bei mackloti ist; daraus darf man wohl schliessen, dass die Unterschenkel bei tuberculatus unbehaart sind. Die Arten der Untergattung Spectrum, deren Schenkel nicht bis zum Fussgelenk dicht behaart sind, vertheilen sich über ein Gebiet, welches im Osten von den nördlichen Salomons-Inseln und den östlichen Karolinen, im Süden ungefähr vom Wendekreis, im Westen vom Meerbusen von Bengalen und im Norden ungefähr vom Wendekreis begrenzt wird. Nur der an den Beinen behaarte leucopterus soll auf den Philippinen diese Grenze nach Süden, der nacktbeinige loochoensis auf den Riukiu-Inseln dieselbe nach Norden überschreiten. Das Vaterland von beiden ist aber nur von Verkäufern angegeben, nicht durch Originalausbeute festgestellt.

In der Färbung unterscheidet sich tuberculatus von den meisten anderen Arten durch das Fehlen eines auffallend gefärbten Halskragens; nur pselaphon, dasymallus, leucopterus, das von insularis, natalis und vielleicht lombocensis sind hierin ähnlich. Die ersten 3 Arten haben behaarte Unterschenkel, scheiden also bei der Betrachtung aus; wir behalten übrig eine Form der Karolinen-Inseln, nördlich vom Aequator, und zwei Formen aus der Südwestecke des Gebietes südlich vom Aequator. Im Gebiss ist tuberculatus mit natalis und lombocensis sehr wenig, mit insularis aber weit mehr verwandt; ich halte es darum für sehr wahrscheinlich, dass das Vaterland von tuberculatus in den Karolinen oder in nächster Nähe derselben gefunden werden wird.

23. Pt. vampyrus Linné. - 1758. Vespertilio vanpyrus Linné partim, Syst. Nat. ed. X. p. 31. - 1775. V. vampyrus Schreber var. A. Säugethiere I. p. 155, Taf. XLIV. - 1802-1804. Pt. niger Desmarest, Nouv. Dict. d'hist. nat. tom. 19 p. 543. - 1804. Vespertilio mauritianus Commerson bei Hermann von Mauritius, Obs. Zool. p. 19. - 1809. Vespertilio caninus Goldfuss. Vergl. Naturbeschr. Säugethiere p. 98, Taf. XI. (V. caninus Blumenb.). - 1810. Pt. vulgaris Is. Geoffroy St. Hilaire von Mauritius und Bourbon, Ann. Mus. XV. p. 92 -93. - 1814. Pt. niger Desmarest, G. Fischer, Zoognosia III. p. 554-557. - 1869. Fitzinger 1. c. p. $65-68$. - 1870. Spectrum vulgare Gray, Cat. Moṇk. Lem. etc. p. $100-101$. - 1878. Dobson 1. c. p. 23. - 1887. Jentink 1. c. p. 252 . - 1888. Jentink 1. c. p. 138. - 1897. Trouessart l. c. p. 77 .

Abbildung des Schädels eines ठึ von Mauritius auf Tafel [9], Fig. 2 als Pt. vulgaris. Hab. Bourbon, Mauritius, Tamatawe auf Madagaskar (P. Z. S. p. 63).

[B. M.] $2 \widehat{\jmath}, 1$ ㅇ Mauritius, Möbius, Sieber. 
24. Pt. livingstonii Gray. - 1866. Pt. livingstonï Gray von der Johanna-Insel, Komoren. - 1888. Jentink l. c. p. 147. - 1897. Trouessart l. c. p. 81.

Abbildung des Schädels eines $\sigma^{t}$ von Johanna auf Tafel [4], Fig. 9-10.

Hab. Johanna-Insel, Komoren.

Dass Pt. livingstoni zur Untergattung Spectrum und nicht zur Untergattung Pteropus gehört, beweist die Kürze und Breite der Schnauze und der Ohren, sowie die Gestalt des vorletzten oberen Molaren.

[B. M.] đ̊ Johanna, Frank.

25. Pt. rodricensis Dobson. - 1878. Pt. rodricensis Dobson von Rodriguez, Cat. Chir. p. 36-37, Taf. III. Fig. 1 (Ohr). - 1879. Dobson, Philos. Transact. CLXVIII. p. 457. - 1881. Anderson, Cat. Mamm. Ind. Mus. I. p. 100.

Hab. Rodriguez.

6. Subgen. Sericonycteris Mtsch. subgen. nov.

Kleine Arten, deren Unterarm nicht länger ist als $120 \mathrm{~cm}$. Hinter dem zweiten Molar höchstens 8 Gaumenfalten; wenn der Unterarm länger ist als $100 \mathrm{~cm}$, so sind die Unterschenkel dicht behaart.

Hab.: Maskarenen, Celebes, Halmahera-Gruppe, Amboina-Gruppe, Banda, Timor, Flores, Ponape und Mortlock in den Karolinen, Bismarck-Archipel, Salomons-Inseln.

Uebersicht der Arten:

Unterschenkel dicht und wollig behaart:

Ohren ganz kurz, im Pelz versteckt . . . . . . rubricollis

Die Ohren ragen aus dem Pelz hervor:

ohne Bindenzeichnung am Kopf:

Bauch grau . . . . . . . . . temmincki

Bauch zimmetfarbig . . . . . . . petersi

mit Bindenzeichnung am Kopf:

eine dunkle Augenbrauenbinde und eine

helle Längsbinde zwischen den Augen . personatus

eine helle Augenbrauenbinde und eine

dunkle Längsbinde zwischen den Augen capistratus

Unterschenkel nackt oder ganz dünn behart:

Nacken dunkler als der Rücken . . . . . . . . . molossinus

Nacken heller als der Rücken. . . . . . . . woodfordi

1. Pt. rubricollis Geoffroy St. Hilaire. - 1810. Pt. rubricollis Geoffroy St. Hilaire von Bourbon, Ann. Mus. XV. p. 93-94. - 1814. Pt. torquatus G. Fischer, Zoognos. p. 533-534. - 1869. Fitzinger 1. c. p. 69-71. - 1870. Spectrum rubricolle Gray 1. c. p. 101. - 1878. Dobson 1. c. p. 24. - 1897. Trouessart l. c. p. 77 .

Abbildung des Kopfes und der Zahnreihe nach einem jungen $q$ von Mauritius auf Tafel [8], Fig. 1.

Hab. Bourbon, Mauritius.

[B. M.] 2 ठ઼ઠ઼, 1 q Bourbon, Pariser Museum; q, q juv. Mauritius, Pariser Museum und Robillard. 
2a. Pt. temmincki Peters. - 1837. Pt. griseus Temminck (nec Geoffroy), von Sama 0, Timor und anderen Inseln in der Nähe, Monogr. Mamm. II. p. $81-82$, Taf. 36, Fig. 12-13 (Schädel). - 1867. Pt. temmincki Peters von Samao, M. B. Akad. Berlin p. 331. - 1869. Pt. griseus Fitzinger partim l. c. p. $61-63$. - 1870. Pt. griseus Gray (nec Geoffroy) partim, l. c. p. 110. - 1878. Pt. temmincki Dobson partim, 1. c. p. 40-41. - 1887. partim Jentink 1. c. p. 254. - 1888. part. Jentink l. c. p. $140-141$ - - 1897. Pt. temmincki partim Trouessart 1. c. p. 79 .

\section{Hab. Samao b. Timor, Timor.}

2b. Pt. petersi Matschie spec. nov. - 1837. Pt. griseus 'Temminck partim, Monogr. Mamm. II. p. $81-82$, Taf. 36, Fig. 14-15. - 1867. Pt. temmincki Peters partim von Amboina, 1. c. p. 331. - 1878. partim Dobson 1. c. p. 40-41. - 1887. partim Jentink 1. c. p. 254. - 1888. partim Jentink 1. c. p. 140-141.

- 1897. partim Trouessart 1. c. p. 79.

Abbildung des Schädels eines $\sigma^{\top}$ von Amboina auf Taf. [6], Fig. 4 als Pt. temmincki. Hab. Amboina, Ceram (?).

Temminck hat auf Tafel 36, Fig. 12-15 als Pt. griseus die Schädel von zwei verschiedenen Arten resp. Abarten dargestellt. Der auf Fig. 14-15 gezeichnete Schädel gehört allerdings einem noch nicht ganz ausgewachsenen Exemplare an,

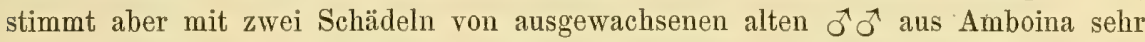
gut überein; eine Vergleichung der Temminck'schen Abbildung mit der unserigen auf Taf. 6, Fig. 4 (als temmincki) beweist dies für eines der Exemplare. Der Schädel, welchen Temminck's Fig. 12 darstellt, ist erheblich grösser, so gross wie derjenige von $P$ t. capistratus und kann meiner :Ausicht nach nicht derselben Art resp. Abart wie die Figuren 14-15 gehören. Dieser Schädel stammt nach Jentink 1887 p. 254 von Timor, der kleinere von Amboina. Dás Ohr des auf der Tafel 35, Fig. 6 bei Temminck abgebildeten Kopfes ist so lang wie die Entfernung vom vorderen Augenrande zur Nasenspitze. Bei unseren Amboina-Exemplaren ist das Ohr kürzer als diese Entfernung. Bei 3 Stücken von Amboina, welche ich vor mir habe, ist der Nacken weissgrau, mehr oder weniger orangefahl überflogen, aber nicht „roux clair" oder gar „beau marron“. Der Unterarm des grössten ơ von Amboina ist $97 \mathrm{~mm}$ lang.... Unsere Exemplare sind ziemlich ausgewachsen, weil die Nasalia an den Schädeln verwachsen sind und die Crista bereits entwickelt ist. Auch Temminck's auf Fig. 15 abgebildeten Schädel ist ziemlich ausgewachsen aus denselben Gründen.

Es erscheint hiernach sehr wahrscheinlich, dass die Sericonycteris von Timor einer anderen Unterart angehört als die S. von Amboina und ich beschränke hierdurch Peter's Namen temmincki auf die Timor- und Samao-Form, während ich die Amboina-Exemplare mit einem neuen Namen belege, Pt. petersi Mtsch. spec. nov. zum Andenken an den um die Chiropteren-Kunde so verdienten Professor Peters.

Dass Pt. tricolor Heude eine auf Flores beschränkte Abart bezeichnet, kann ich nur vermuthen.' In der Beschreibung, welche Heude giebt, wird das Kinn als "roux clair" und der Hals als "blanc un peu teinté de roux" angegeben und auf der Tafel V, Fig. 7 ist der zweite obere Praemolar mehr oval, nicht wie bei den Amboina-Exemplaren seitlich comprimirt; der erste untere Praemolar ist etwas grösser als der letzte untere Molar, während bei dem von Temminck abgebildeten Timor-Schädel das Umgekehrte der Fall ist. 
Diese erheblichen Unterschiede sprechen dafür, dass Pt. tricolor Heude von Flores eine geographische Abart von Pt. temmincki darstellt. Da Gray 1870 schon einen Pt. tricolor beschrieben hat, so darf Heude's Name nicht verwendet werden. Ich schlage vor, ihn in Pteropus (Sericonycteris) heudei Mtsch." nov. nom. umzuändern.

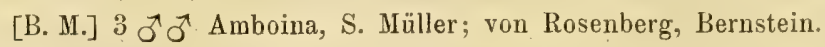

2c. Pt. heudei Mtsch. nov. nom. - 1896. Pt. tricolor Heude von Flores, Mém. Hist. Nat. Emp. Chinois III. p. 176 Tafel V, Fig. 7 (Zahnreihe).

\section{Hab. Flores.}

„Le seul sujet, que j'ai de cette jolie espèce, mesure 15 centimêtres. La tête osseuse est moitié plus petit que celle de l'édulis. La tête et le menton sont roux clair; le cou est blanc un peu teinté de roux; le dos est gris. Les parties correspondantes inférieures ont les mêmes teintes. Pelage doux et soyeux. Obtenu à Flores, sur un figuier, au clair de la lune, grâce à l'adresse du maître d'écoel de la mission de Coting." Heude l. c.

3. Pt. personatus Temminck. - 1827. Pt. per'sonatus Temminck von 'Ternate, Monogr. Mammal. I. p. 189-190. - 1869. Fitzinger 1. c. p. $64-65$ - - 1878. Dobson 1. c. p. $38-39$. - 1897. Trouessart 1. c. p. 79. Fig. 5 .

Abbildung des Schädels nach einem Leidener Exemplar von Ternate auf Taf. [7],

Hab. Ternate, Morotai (Jentink 1888 p. 140), Gorontalo auf Celebes (B. MI.).

Jentink führt unter d an: Mâle semi-adulte monté, un des types de l'espèce. Ceram. Des collections de M. Reinwardt. (Cr. G. du Cat. Ost.) Temminck sagt: Patrie. M. Reinwardt a rapporté les deux individus mentionnés de l'ile de Ternate. Im Leidener Museum ist nur noch ein zweites von Reinwardt gesammeltes Exemplar dieser Art und dieses trägt den Fundort Ternate.

Auf Ceram lebt nach Dobson Pt. temmincki resp. mein Pt. petersi, eine mit personatus nahe verwandte Form. So lange bis Pt. personatus neuerdings auf Ceram wieder aufgefunden wird, möchte ich Ceram als Vaterland dieser Art nicht nennen.

4. Pt. capistrata (Peters). - 1876. Pt. capistratus Peters von Neu-Mecklenburg, M. B. Akad. Berlin p. 316-317. Tafel (Thier). - 1878. Dobson 1. c. p. 39-40. - Trouessart 1. c. p 79.

\section{Hab. Neu-Pommern, Neu-Lauenburg, Neu-Mecklenburg.}

Abbildung des Thieres auf Tafel [2] nach Exemplaren von Neu-Mecklenburg; Abbildung des Schädels eines 우 von dort auf Tafel [3], Fig. 3.

[B. M.] $\sigma^{7}$ 오 Neu-Mecklenburg, S. M. S. Gazelle; 우, 우 juv. Neu-Lauenburg, Hübner; ऽ, 3 우우 Neu-Pommern, Finsch. - [Dresd. Mus.] 우 Kiniguan, Gazellen-Halbinsel, Neupommern, Geisler.

5. Pt. molossinus (Temminck). - 1853. Pt. molossinus Temminck von unbekanntem Fundorte. Esquiss. Zool. Cote de Guiné I. P. 62-64. - 1878. Dobson 1. c. p. 30-31. - 1883. von Ponape, Carolinen. Peters Sitzb. Ges. nat. Fr. Berlin p. 2. - 1882. Pt. breviceps Thomas von Ponape, P. Z. S. p. 756 T. LV. - 1887. Pt. molossinus = breviceps Thomas p. 322, Anmerkung. - 1897. 'Trouessart 1. c. p. 78. 

Fig. 5 .

Abbildung des Schädels, wahrscheinlich nach dem Original-Exemplare auf Taf. [6],

Hab. Ponape, Carolinen.

[B. M.] $7 \sigma^{\top} \sigma^{\top}, 5$ 우우 Ponape, Finsch und Mus. Godeffroy.

6. Pt. woodfordi (Thomas). - 1888. Pt. woodfordi Thomas von Aola, Guadalcanar, Salomons-Inseln. Ann. Mag. N. H. (6) I pag. 156, - 1888. P. Z. S. p. 472-473, 483 T. XX. Fig. 1 (Kopf), T. XXI. Fig, 1 (Schädel). — Trouessart l. c. p. 78 .

Hab. Aola, Guadaleanar, Salomons-Inseln.

[Dresd. M.] Aola, Woodford.

\section{Genus. Styloctenium Mtsch, gen, nov.}

Obere Incisiven kegelförmig wie bei Acerodon, die mittleren grösser als die äusseren. Nur 2 untere Incisiven; zweiter oberer Praemolar sehr hoch, nur um $1 / 3$ niedriger als der Eckzahn. Jochbogen sehr an den Schädel angedrückt, nicht geschweift. In den äusseren Merkmalen sehr ähnlich der Gattung Sericonycteris.

Typus: Pteropus wallacei Gray.

Hab. Celebes.

Nur eine Art: St. wallacei Gray. - 1870. Pt. wallacei Gray von Makassar, Cat. Monk. Lem. Fruit-eating Bats. p. 111-112 (Abb. des Kopfes). - 1878. Dobson l. c. p. 40. - 1883. Jentink, Not. Leyd. Mus. V. p. 172-173. 1888. Jentink l. c. p. 170.

Abbildung des Schädels nach einem 오 aus der Minahassa auf Taf. [4], Fig. 1-4. Hab. Celebes, Makassar (Dobson), Amoerang, Nord-Celebes (Jentink 1. c.).

[B. M.] \& Minahassa, A. B. Meyer. [Dresd. M.] § Lotta, Minahassa, NordCelebes; Mt. Masarang, Nord-Celebes, Hose - [Sarasin-Samml.] $\sigma^{\top}{ }^{\top}$ q Tomohon, Celebes, Gebr. Sarasin. 


\section{Genus. Epomophorus Bennett.}

1835. Epomophorus Bennett, P.Z. S. p. 149; Trans. Z. S. II. p. 31-38, Tafel 6 und 7.

1869. L. J. Fitzinger in Sitzb. Ak. Wiss. Wien LX, 1. Abth. November p. 1-21. - 1878. G. E. Dobson, Catalogue of the Chiroptera p. 4-15, 552, Tafel I und II. - 1887. F. A. Jentink in Mus. d'Hist. Nat. Pays-Bas IX. p. 251 -252. - 1888. F. A. Jentink I. c. XII p. 136-138. - 1889. J. V. Barboza du Bocage, Jorn. Scienc. Math. Phys. Nat. 2. Ser. I. p. 1-4. - 1894. Matschie, Mitth. Geograph. Ges. und Naturh. Mus. Lübeck II. Ser., Heft 7 und 8, p. 132-133. - 1896. E. de Pousargues, Ann. Sc. Nat. Zool. III. p. 250-256. - 1897. E. L. Trouessart, Cat. Mamm. Nov. Ed. I. p. 88-89. - 1898. Bocage, 1. c. V. p. 135-137.

Grosse Fledermäuse mit sehr kurzem, und dann von der Schwanzflughaut vollständig getrenntem Schwanz oder ohne einen solchen; der Zeigefinger trägt eine Kralle. Der Rand des Ohres bildet einen ovalen Ring, da sich der Aussenrand mit dem Innenrand an der Ansatzstelle des Ohres an den Kopf berührt. Die Flughaut setzt sich an den Rücken der zweiten. Zehe an. Schnauze hundeartig. Der Metacarpus des Mittelfingers ist ungefähr so lang wie der Zeigefinger; der Daumen ist zum Theil von der Flughaut umhüllt. Vor und hinter der Ohrwurzel je ein Büschel weisser Haare. Die Flügel sind ziemlich weit hinten am Rumpf angesetzt. Gebiss: $\frac{2,1,1,2}{2,1,2,3}$. Bei der Untergattung Epomops fallen alten Thieren die beiden äusseren oberen Schneidezähne häufig aus, so dass nur noch 2 Incisivi im Oberkiefer stehen.

Die oberen Schneidezähne sind von den Eckzähnen durch eine Lücke getrennt und stehen in einem Bogen. Die Backenzähne sind stumpfhöckerig mit einer Längsfurche auf der Krone. Zwischen dem Eckzahn und dem 2. Praemolar ist eine Lücke, welche bei den mir bekannten Schädeln desto breiter erscheint, je älter das Thier ist. Der knöcherne Gaumen reicht weit über die Molarenreihe hinaus. - Der Schädel ist sehr flach. Die Praemaxillaria sind vorn durch eine Naht verbunden. Die Saugwarzen befinden sich an den Seiten der Brust etwas unter der Achselgrube. Ruthe des Männchens ohne Penisknochen. Auf der Schulter sind bei den Männchen der meisten Arten eigenthümliche Drüsentaschen vorhanden, welche mit, langen weissen oder gelben Haaren ausgekleidet sind.

Die Nasenlöcher sind wulstig umrandet; zwischen denselben verläuft eine tiefe Furche über die Oberlippe herunter, welche letztere namentlich bei alten Männchen sebr dick ist, wie bei einer Bulldogge lappig herabhängt und auf der Innenseite einige zackig hervortretenden Schleimhautfalten aufweist. 
Die Weibchen sind im allgemeinen kleiner als die Männchen und haben einen kürzeren Kopf mit weniger wulstiger Oberlippe.

Die Färbung ist bei allen bekannten Arten ungefähr die gleiche, und wir finden bei den Epomophorus nicht die Mannigfaltigkeit in den Farbenkleidern wie bei den Pteropus. Die Epomophorus sind isabellgrau bis dunkel zimmetbraun, zuweilen ist auf der Unterseite ein weisses Feld scharf abgesetzt.

In ihrer Lebensweise erinnern die Ohrbüschel-Flughunde sehr an die fliegenden Hunde von Süd-Asien (Pteropus). Wir haben zwar erst spärliche Nachrichten über diese Thiere, aber alle Beobachter stimmen darin überein; dass die Epomophorus Bäume bewohnen und saftige Früchte mit Vorliebe verzehren, bei welcher Thätigkeit ihnen die wulstigen Lippen jedenfalls gute Dienste leisten (vergl. P. Z. S. 1881, p. 685-693).

Diese Flughunde hängen bei Tage an Zweigen, um gegen Abend ihrer Nahrung nachzugehen. Die Stimme klingt eigenthümlich metallisch (nach Böhm), quakend (nach Büttikofer), gackernd (nach Sjöstedt).

Die Gattung Epomophorus ersetzt in Afrika die über die gesammten übrigen Theile der altweltlichen Tropen verbreitete Gattung Pteropus.

Es sind bis jetzt 20 Species von Epomophorus beschrieben worden; von diesen hat Trouessart 189711 als Arten angenommen. Ich nehme 15 von den bekannten Arten an, beschreibe 6 neue und mache auf weitere zwei Arten, die jedenfalls neu sind, aufmerksam.

Versuche, die Gattung Epomophorus in Untergattungen zu zerlegen, sind schon wiederholt gemacht worden.

Im Jahre 1861 hat Allen eine Epomophorus ähnliche Gattung: Hypsignathus aufgestellt, welche Murray ein Jahr später noch einmal als Sphyrocephalus beschrieb. Gray bildete 1870 einen Tribus: Epomophorina aus den Gattungen: Hypsignathus, Epomophorus und einer von ihm neu aufgestellten Gruppe: Epomops.

Dobson hat später Hypsignathus und Epomophorus als Untergattungen von Epomophorus in die Litteratur eingeführt, konnte sich aber nicht davon überzengen, dass Epomops als Subgenus abgetrennt werden müsse. Ich nehme 4 Untergruppen an:

Die Untergattung: Hypsignathus Allen ist ausgezeichnet durch das Fehlen der Schultertasche bei beiden Geschlechtern und durch das Vorhandensein von einer Falte jederseits in der Oberlippe unter dem Nasenloch. Der Schädel ist über dem letzten Molar ungefähr eben so hoch als über der Canine; der vorletzte, untere Molar hat aussen drei, innen einen, an der Krone gewöhnlich getheilten Höcker. Hierher gehört: Ep. haldemani.

Die Untergattung: Epomophorus Bennett umfasst Arten, welche eine lange Schnauze haben und deren Lippen nicht gefaltet sind. Die Entfernung zwischen dem vorderen Augenrande und der Nasenspitze ist viel grösser als die Breite des Gesichtes an den Mundwinkeln. Auf dem Plagiopatagium, der Ellenbogen-Flughaut, wird der vom Ellenbogen zur Spitze des fünften Fingers verlaufende Strang von höchstens 32 längeren, stark hervortretenden Nebensträngen durchschnitten. Der Schwanz ist immer deutlich fühlbar. Das hintere Ende des knöchernen Gaumens verläuft nicht ungefähr in derselben Ebene wie der zwischen den Molaren befindliche Theil, sondern ist nach oben etwas aufgerollt. Der letzte obere Molar hat, falls er noch nicht zu sehr abgekaut ist, eine Ausbuchtung am hinteren. Ende. - Hierher gehören Ep. gambianus, macrocephalus, wahlbergi, angolensis, crypturus, anurus, labiatus, minor und schoensis, sowie einige neu zu benennende Formen.

Die Untergattung: Epomops Gray enthält Arten, deren Schnauze kurz und deren Lippen ungefaltet sind. Die Entfernung zwischen dem vorderen Augenrande und der Nasenspitze ist ungefähr so gross wie die Breite des Gesichtes an den Mundwinkeln. Auf dem Plagiopatagium wird der vom Ellenbogen zur Spitze des fünften Fingers verlaufende Strang 
mindestens von 36 längeren, dicht aneinander liegenden Nebensträngen durchschnitten. Der Schwanz ist nicht zu fühlen. Das hintere Ende des knöchernen Gaumens verläuft ungefähr in derselben Ebene wie sein vorderer Theil und ist nicht nach oben aufgerollt. Der letzte obere Molar hat eine gerade Aussenkante und ist hinten nicht breiter als vorn. Hierher rechne ich Ep. comptus, Ep. franqueti und Ep. dobsoni.

Es bleiben noch zwei Arten für die Betrachtung übrig, Ep. pusillus und Ep. veldkampi. Beides sind sehr kleine Formen, deren knöchernen Gaumen wie bei Epomops gebildet ist und deren Plagiopatagium an dasjenige von Epomophorus s. str. erinnert.

Ep. pusillus hat die Kopfbildung von Epomops, Ep. veldkampi diejenige von Epomophorus s. str. Bei Ep. pusillus ist die Molaren-Reihe viel länger als die Entfernung zwischen den Spitzen der Caninen und der letzte obere Molar reicht weit über das Foramen infraorbitale nach hinten. Bei Ep. veldkampi ist die Molaren-Reihe so lang wie die Entfernung der Spitzen der Caninen von einander und der letzte obere Molar reicht nur bis zur Höhe des. Vorderrandes des Foramen infraorbitale.

Ich 'kann diese beiden kleinen Arten in keiner der oben gekennzeichneten Untergattungen unterbringen; es sind die Unterschiede, welche ihre Schädel darbieten, sogar so gross, dass man sie in zwei besonderen Untergattungen trennen muss.

Ich nehme für Ep. pusillus den Untergattungsnamen, „Micropteropus" (von $\mu$ cxpós = klein und Pteropus), für Ep. veldkampi den Untergattungsnamen: Nanonycteris (von vãvos = Zwerg and v'sxepıs = Fledermaus).

Die Gaumenfalten sind schon von Dobson zur Unterscheidung der' einzelnen Arten gebraucht worden; ich habe bei der Uebersicht der Gattungen und Arten auf die Merkmale aufmerksam gemacht, welche sich bei der Berücksichtigung der Gaumenfalten ergeben.

Hab.: Afrika, südlich von der. Sahara, nach Süden bis Port Elisabeth im Caplande.

\section{Uebersicht der Untergattungen.}

Vom unteren Rande der Nasenlöcher verläuft eine stark hervor-

tretende Hautfalte über die Oberlippe bis zum Lippenrande:

Oberlippe ohne eine stark hervortretende Hautfalte:

Unterarm bei Thieren, welche das vollständige Gebiss haben, länger als $60 \mathrm{~mm}$ :

Die Entfernung zwischen dem vorderen Augenwinkel und der Nasenspitze ist viel grösser als die Breite des Gesichtes an den Mundwinkeln; Schwanz deutlich fühlbar; Plagiopatagium mit. höchstens 32 längeren Strängen, welche den vom Ellenbogen zur. Spitze des fünften Fingers verlaufenden Strang durchschneiden:

Die Entfernung zwischen dem vorderen Augenwinkel und der Nasenspitze ist ungefähr so gross wie die Breite des Gesichtes an den Mundwinkeln; der Schwanz ist nicht zu fühlen; auf dem Plagiopatagium wird der vom Ellenbogen zur Spitze des fünften Fingers verlaufende Strang von mindestens 36 längeren Strängen durchschnitten: . . . Epomops Gray p. 56 
Unterarm höchstens $60 \mathrm{~mm}$ lang:

Die Entfernung zwischen dem vorderen Augenwinkel und der Nasenspitze ist ungefähr so gross wie die Breite des Gesichtes an den Mundwinkeln; Gaumen mit einer vorn gegabelten Längsgrube . . . . . . .

Die Entfernung zwischen dem vorderen Augenwinkel und der Nasenspitze ist viel grösser als die Breite des Gesichtes an den Mundwinkeln; Gaumen mit Querwülsten; MolarenReihe nur so lang wie die Entfernung zwischen den Spitzen der oberen Caninen . . Nanonycteris Mtsch. p. 58.

Uebersicht der geographischen Verbreitung.

\begin{tabular}{|c|c|c|c|c|c|}
\hline & Hypsignathus & Epomophorus & Epomops & Micropteropus & Nanonycteris \\
\hline Gambia & haldemani & macrocephalus & & - & - \\
\hline West-Guinea & haldemani & büttikoferi & franqueti & pusillus & veldkampi \\
\hline Mittel-Guinea & haldemani & . zechi & franqueti $\stackrel{\approx}{\Xi}$ & pusillus & veldkampi \\
\hline *Unterer Niger & haldemani & ? & ? & pusillus & - \\
\hline Nieder-Guinea & haldemani & zenkeri & franqueti $\int \tilde{\Xi}_{0}$ & pusillus & - \\
\hline Congo & haldemani & zenkeri & comptus & pusillus & 一 \\
\hline Loanda & - & zenkeri & dobsoni & pusillus. & - \\
\hline Benguella & - & angolensis & - & - & - \\
\hline Orange & - & ? & - & - & - \\
\hline West-Capland & - & ? & - & - & - \\
\hline Ost-Capland & - & wahlbergi & - & - & - \\
\hline Limpopo & - & ? & - & - & - \\
\hline Ngami & - & spec. nov.? & - & - & - \\
\hline Zambese & - & crypturus & - & - & - \\
\hline Mero & - & ? & - & $\therefore$ & - \\
\hline Mossambik & - & $?$ & - & - & - \\
\hline Zanzibar-Küste & - & stuhlmanni & - & - & - \\
\hline Massai-Land & - & $?$ & - & - & - \\
\hline Málagarasi & - & minor & - & - & - \\
\hline Seeen-Gebiet & - & aff. schoensis & $\begin{array}{l}\text { comptus u. } \\
\text { franqueti }\end{array}$ & pusillus & - \\
\hline Ukambani & - & neumanni & -1 & - & - \\
\hline Somali & - & ? & - & - & - \\
\hline Rudolf See & - & schoensis & - & - & - \\
\hline Gazellen-Fluss & - & anurus & - & pusillus & - \\
\hline Bahr el Abiad & - & labiatus & - & - & - \\
\hline Erythraea & - & doriae & - & - & - \\
\hline Berbera-Küste & - & ? & - & - & - \\
\hline Tschad-See & - & spec. nov.? & - & - & - \\
\hline Oberer Niger & - & $?$ & - & - & - \\
\hline Senegal & - & gambianus & - & - & - \\
\hline
\end{tabular}

* Vom Benue-Gebiet haben wir noch keine Nachrichten über Epomophorus. 


\section{Die zoogeographischen Gebiete der aethiopischen Region.}

Reichenow hat das aethiopische Gebiet eingetheilt in ein westliches Waldgebiet und in ein Steppengebiet, welches den Nordwesten, Nordosten, Osten, Südosten, Süden und Südwesten von Afrika umfasst. Das. Waldgebiet erstreckt sich von der Wasserscheide zwischen dem Senegal und Gambia bis herunter zu derjenigen zwischen Cuanza und Catumbella und rejcht nach Norden in dem Nigerbecken bis ungefähr zur Einmündung des Benue, nach Westen bis zum Victoria-Nyansa, dessen Nord- und Ostrand bis zum Speke-Golf herunter zum westlichen Waldgebiet gehören. Im Südosten rechnet Reichenow das Waldgebiet bis in die Nähe des Westrandes des Nyassa. Alle Gegenden, welche nicht zum Waldgebiet gehören, bilden das Steppengebiet, welches Reichenow in vier grössere Untergebiete, ein südliches, ein südwestliches, ein nordöstliches und ein nordwestliches eintheilt.

Ich habe, wie die auf Seite 37 stehende Verbreitungstabelle ergiebt, die aethiopische Region in eine grössere Anzahl kleinerer Gebiete zerlegt, eine Eintheilung, welche in dieser Weise wohl noch nicht versucht worden ist.

Ich bin der Ueberzeugung, dass zwei Faunengebiete nur dann scharfe Grenzen zeigen werden, wenn sie durch sehr hohe Gebirgszüge getrennt sind. Im allgemeinen wird zwischen je zwei Thiergebieten eine Uebergangszone vorhanden sein, in welcher die Arten beider Gebiete neben einander leben werden, nicht überall, aber in gewissen Gegenden. Die eine Art wird ihren für sie geeigneten Lebensbedingungen entsprechend weiter verbreitet sein als eine andere; wir werden aber immer für je-zwei Faunengebiete Gegenden nachweisen können, in welchen nur die für das eine Gebiet charakteristischen Thiere vorkommen, ebenso solche, in denen nur die für das andere Gebiet charakteristischen Thiere leben, und dazwischen werden wir eine Zone unterscheiden, in welcher die eine Fauna, und eine andere, in welcher die andere Fauna überwiegt. Es können auch Fälle eintreten, wo in einem Mischgebiet in den Wäldern die Fauna des einen Gebietes, in den Steppen diejenige des anderen gefunden wird.

Nun wissen wir, dass eine grosse Anzahl von Gattungen nur im Westen lebt, während eine ebenso grosse dort fehlt und nur im Steppengebiet auftritt. Wir haben also an der Guinea-Küste eine wesentlich andere Fauna als z. B. an der Küste von DeutschOst-Afrika. Es ergiebt sich hierdurch in sehr natürlicher Weise die Eintheilung der aethiopischen Region in zwei Faunengebiete. Nur darf man nicht alles Land, in welchem westliche Formen auftreten, zum Westen rechnen; denn ebenso gut könnte man dann alles Gebiet, in welchem Steppen-Formen auftreten, zum Steppengebiet rechnen.

Die Inlandsgrenze der westlichen Gattungen giebt uns eine Linie, welche dasjenige Gebiet nach Westen abschliesst, in dem die ungemischte Steppenfauna auftritt. In ähnlicher Weise können wir die Westgrenze resp. Südgrenze im Norden und Nordgrenze im Süden für die Gattungen festlegen, welche z. B. an der Goldküste fehlen. Wir erhalten so eine zweite Linie, welche dasjenige Gebiet nach dem Inlande zu begrenzt, in welchem nur die westlichen Gattungen ungemischt vorhanden sind. Zwischen beiden Linien wird ein Gebiet liegen, welches sowohl Einflüsse der westlichen Fauna als der Steppenfauna zeigt.

Reichenow hat die erste Grenzlinie auf Grund seiner ornithologischen Forschungen gezogen; für die Säugethiere gilt sie genau so wie für die Vögel. Ueber den Verlauf der zweiten Linie findet man in der Litteratur sehr wenig.

Ich habe die Erfahrung gemacht, dass die Faunengebiete sehr innige Beziehungen zu den Meeresgebieten haben. Soweit die Flüsse in der alten Welt z. B. nach Norden zum Eismeer und Nord-Atlantik strömen, ist die Thierwelt eine ziemlich gleichartige; südlich davon breitet sich ein abflussloses Gebiet aus, in welchem die Wasserläufe in das 
kaspische Meer, den Aral-See, Balkasch-See, das Lob-Nor u..s.w. sich ergiessen. Auch dieses Gebiet hat seine eigenthümliche Fauna. Sobald man die Wasserscheide südlich von diesem abflusslosen Gebiet überschritten hat, gelangt man wiederum in neue Gebiete, welche je nach dem Meere, zu dem sie abwässern, ein verschiedene Fauna zeigen.

Wenn man eine Art an 3 oder 4 Stellen innerhalb eines Flussgebietes gefunden hat, so kann man im allgemeinen mit einer grossen Wahrscheinlichkeit annehmen, dass sic an geeigneten Stellen überall innerhalb dieses Flussgebietes vorkommt; wenn ein Strom an seiner Mündung eine andere Fauna zeigt, als in seinem Oberlaufe, so liegt der Verdacht nahe, dass er aus zwei verschiedenen Flüssen, die ursprünglich einen ganz anderen Verlauf hatten, entstanden ist dadurch, dass die Wassermassen des einen an irgend einer Stelle über die flache Wasserscheide in das Gebiet des anderen eingebrochen sind. Dass so etwas vorkommen kann, sehen wir an den Veränderungen, welche die norddeutsche Tiefebene einst erlitten hat. Dort fliessen die Oder und Weichsel heute in die Ostsee, während sie früher ihre Gewässer in die Elbe sendeten. In Afrika fliessen der Niger und der Nil durch verschiedene Faunen-Gebiete. Man kann also vermuthen, dass ihr jetziger Lauf ein anderer ist als der ihnen ursprünglich eigene.

Die Wasserscheide, auf welcher die in den Golf von Guinea sich ergiessenden Flüsse entspringen, schneidet den Niger ungefähr an der Mündung des Benue. Hier, wo der Niger eimen scharfen Knick bildet, muss eine Bifurkation nachgewiesen werden. Ebenso wird mán unter Berücksichtigung der geologischen Verhältnisse auch am Victoria-Nyansa und am Nil einmal eine Erklärung für die merkwürdige Zusammensetzung der dortigen Thierwelt finden können.

Ich habe die einzelnen Gebiete, in welche ich die aethiopische Region zerlege, möglichst klein genommen, weil ich glaube, dass es leichter ist, zunächst zu trennen und dann zu vereinigen, als umgekehrt.

Wieweit das Verbreitungsgebiet der westlichen Gattungen ohne Beimischung von solchen ist, die dem Steppengebiet eigenthümlich sind, das wissen wir noch nicht. Vorläufig rechne ich zu dem eigentlichen westlichen Faunengebiet die ersten 4.Gebiete und das 6. und 7. Gebiet meiner Uebersicht:

Unter dem Namen

1. Gambia fasse ich Süd-Senegambien zusammen mit dem portugiesischen und französischen Guinea. Die Grenze wird gebildet von der Wasserscheide zwischen Senegal und Gambia und im östlichen Fouta Djalon von derjenigen zwischen dem Senegal und den Küstenflüssen. In der Richtung auf Sierra Leone wird vielleicht die Wasserscheide nördlich von Rio dos Carceres die Grenze bilden.

2. West-Guinea nenne ich den Theil des politischen Ober-Guinea, welcher Sierra Le one, Liberia und einen Theil der Elfenbeinküste umfasst. West-Guinea wird nach dem Inlande wahrscheinlich durch die Wasserscheide begrenzt, auf welchem die Küstenflüsse entspringen. Wo an der Elfenbeinküste die Grenze liegt, wissen wir noch nicht.

3. Mittel-Guinea umfasst den östlichen Theil der Elfenbeinküste, die Goldkïste, Togo und Dahome, nach Norden bis zur Wasserscheide zwischen den Küstenflüssen und den Zuflüssen des Niger. Die Ost-Grenze dieses Gebietes ist nicht festgestellt.

4. Der untere Niger bis zum Benue bildet ein viertes Faunengebiet. Die Grenze gegen Mittel-Guinea ist nicht festgestellt.

Nach Norden scheint die Wasserscheide zivischen dem Ocean und den NigerBenue-Zuflüssen dieses Gebiet zu begrenzen. Gegen Nieder-Guinea wird wohl die Wasserscheide südlich vom Cross-Fluss und nördlich vom Mbam die Grenze bilden.

5. Der Benue wird ein Mischgebiet zwischen der westlichen und Sudan-Fauna darstellen. 
6. Nieder-Guinea umfasst den grösseren Theil von Kamerun und Gabun. Bei Victoria mischt sich die Fauna von Ober- und Nieder-Guinea. Vom Kamerungebirge erstreckt sich dieses Thiergebiet nach Süden bis zu den Quellen der Kuilu-Zuflüsse, nach Osten bis zu der Wasserscheide gegen die Congo- und Schari-Zuflüsse.

7. Das Congo-Gebiet reicht soweit, wie die Gewässer zum Congo fliessen, und umfasst den gesammten Congo-Stat mit Ausnahme der zum Tanganyika und zum Nero-See abwässernden Gebiete, den süd-östlichen Theil von Kamerun, das Hinterland des CongoFrançais, den nord-östlichen Theil von Loanda und das Lunda-Reich.

8. Loanda wird im Norden von der Wasserscheide südlich des Congo in Nord-Angola, gegen Osten von den Quellgebieten der Congo-Zuflüsse, nach Süden von der Wasserscheide zwischen dem Cuanza und den Zambese-, Okawango = und CuneneZuflüssen begrenzt.

In Loanda überwiegen, wie es scheint, die westlichen Arten; es treten aber auch schon südliche auf.

9. Benguella umfasst: Benguella, Mossamedes und die Küste von Deutsch-Südwest-Afrika bis herunter zur Lüderitzbucht. Die Inlandsgrenze verläuft im Norden in der Nähe des Catumbella, nach Osten auf der Wasserscheide gegen die Okawango- und OrangeZuflüsse.

10. Das Orange-Gebiet ist beschränkt auf diejenigen Gegenden, welche zum OrangeFluss abwässern. Es schliesst sich in Gross-Namaland an das vorige Gebiet an, erstreckt sich nach Nordosten bis zu den Quellgebieten der Nosob-Zuflüsse, reicht auf der Wasserscheide zwischen Vaal und Limpopo bis zu den Quellgebieten der südostafrikanischen Küstenflüsse und wird nach Süden begrenzt von den Gebirgen, auf welchen die südlichen Zuflüsse des Orange entspringen.

11. West-Capland nenne ich den Theil der Cap-Colonie, welcher südlich von den Zuflüssen des Orange-Flusses gelegen ist und seine Ostgrenze ungefähr in der Höhe des grossen Winterberges und am Grossen Fisch-Fluss hat.

12. Ost-Capland erstreckt sich östlich von den Zuflüssen des Orange-Flusses über Kaffraria, Natal, Zulu-Land und Swazi-Land bis ungefähr zur Delagoa Bay. Das

13. Limpopo-Gebiet reicht nach Norden bis an die Wasserscheide gegen den Sabi.

14. Als Ngami-Gebiet bezeichne ich das abflusslose Gebiet, welches nach Westen von den Quellengebieten der atlantischen Küstenflüsse, nach Süden von der Wasserscheide gegen die Orange-Zuflüsse, nach Osten von derjenigen, auf der die Limpopo-Zuflüsse entspringen, nach Norden von der Wasserscheide gegen den Zambese begrenzt wird.

15. Das Zambese-Gebiet umfasst alle zum Zambese abwässernden Gegenden und die Küste vom Sabi nach Norden bis zur Zambese-Mündung.

16. Das Mero-Gebiet ist das abflusslose Gebiet zwischen den Congo- und Zambese-Zuflüssen.

17. Als Mossambik bezeichne ich das Gebiet der Küstenflüsse nördlich von Zambese bis nördlich vom Rowuma in Deutsch-0st-Afrika.

18. Zanzibar-Küste nenne ich das Gebiet der Küstenflüsse von Deutsch-Ost-Afrika zwischen der Wasserscheide, auf welcher die südlichen Zuflüsse des Rufidji und Ruaha entstehen, bis zum Usambara-Hochlande, nach Westen bis zur Wasserscheide, auf welcher die Küstenflüsse entspringen.

19. Als Massai-Land bezeichne ich das abflusslose Gebiet, welches westlich von den Quellen der Küstenflüsse liegt und nicht nur die eigentlichen Massai-Hochländer, sondern auch das Eyassi-Gebiet umfasst. Nach Westen bilden die Zuflüsse des Malagarasi und des südlichen und östlichen Nyansa die Grenze, nach Norden die zum Naiwascha- und Baringo-System gehörigen Flüsse. 
20. Unter dem Namen Malagarasi fasse ich die Gegenden zusammen, welche von Osten und Norden her in den Tanganyika und von Süden her in den Nyạnsa abwässern.

21. Als Seen-Gebiet vereinige ich die Länder, welche zum Albert-See, AlbertEdward-See und zum grösseren Theile des Nyansa abwässern. Dieses Gebiet wird gegen Westen von den Quellgebieten der Congo-Zuflüsse begrenzt, gegen Norden von der Wasserscheide, auf welcher die in den Nordrand des Nyansa stürzenden Flüsse entspringen, nach Osten von der Wasserscheide, auf. welcher die in den Ostrand des Nyansa sich ergiessenden Flüsse entstehen, und nach Süden von einer Linie, die ungefähr südlich vom Ngare Dobasch über Ukerewe und Bukome bis zu der Wasserscheide gegen die Tanganyika-Zuflüsse sich erstreckt.

22. Ukambani umfasst Britisch-Ost-Afrika und den nördlichsten Theil des Küstengẹbietes von Deutsch-Ost-Afrika, nördlich von den Usambara-Hochländern nach Westen bis zu den Qnellgebieten der Küstenflüsse, nach Norden bis zuir Wasserscheide nördlich vom Tana.

23. Das Somali-Plateau schliesst sich nach Norden an Ukambani an, reicht nach Westen bis zu der Ostgrenze des abflusslosen Gebietes, nach Norden bis zur Wasserscheide gegen die Zuflüsse des Golfes von Aden.

24. Als das Rudolf-See-Gebiet bezeichne ich das abflusslose Gebiet nördlich von Guasso Nyiro des Massai-Landes, östlich bis zu den Quellgebieten der Flüsse, welche das Somali-Plateau durchziehen, westlich bis zur Wasserscheide gegen den Bahr el Gebel, nach Norden bis zum Tana-See und Siemen-Gebirge. Der Rudolf-See, der StephanieSee und Schoa liegen in diesem Gebiet.

Auf den Karten fliesst der Bahr el Azrek vom Tana-See erst nach Süden und dann im grossen Bogen nach Norden. Er tritt dann in ein neues Faunengebiet ein, das sich bis Sennaar erstreckt. Hier scheint wieder eine nachträgliche Veränderung der Flussläufe stattgefunden zu haben, da der Oberlauf der Bahr el Azrek eine andere Fauna als der Unterlauf besitzt.

25. Das Gebiet des "Gazellen-Flusses“ von den Quellgebieten der Congo-Zuflüsse bis zu dem abflusslosen Gebiet in. Kordofan und Dar-Fur, nach Westen bis zur Wasserscheide gegen die Tschad-See-Zuflüsse, nach Osten bis zur Wasserscheide gegen den Bahr el Azrek bildet wieder ein einheitliches Faunen-Gebiet.

26. Der Bahr el Abiad von der Einmündung des Gazellen-Flusses nach Norden bis Dongola, der Atbara mit seinen Zuflüssen und der Bahr el Azrek nach seinem Austritt aus den abessynischen Gebirgen bewässern ein Gebiet, welches nach Osten von der Wasserscheide gegen die Küstenflüsse des Rothen Meeres, nach Süden von der Wasserscheide gegen die westlichen Zuflüsse des Bahr el Azrek und des Atbara begrenzt wird.

27. Die Erythraea reicht als zoologisches Gebiet von der Nordgrenze der aethiopischen Region am Rothen Meer nach Süden bis zur Wasserscheide gegen den Haiwasch, nach Westen bis zur Waśserscheide gegen die Zuflüsse des Bahr el Azrek.

28. Die Berbera-Küste schliesst sich an dieses Gebiet nach Osten an und umschliesst die Länder, in denen die Flüsse in den Golf von Aden stürzen.

29. Das Tschad-See-Gebiet umfasst die von den Zuflüssen des Tschad-See bewässerten Gegenden.

30. Das Gebiet des oberen Niger reicht nach Süden bis an die Nordgrenze des Gebietes des unteren Niger und von Mittel- und West-Guinea nach Norden bis zur Nordgrenze der aethiopischen Region, nach Osten bis : zu den Quellgebieten der Tschad-See-Zuflüsse, nach Westen bis zur Wasserscheide gegen den Senegal.

31. Das Senegal-Gebiet wird nach Süden vom Gambia-Gebiet, nach Osten vom Gebiet des oberen Niger begrenzt. 


\section{Subgen. Hypsignathus Allen.}

1861. Allen, Proc. Acad. Nat. Sc. Philadelphia p. 156. - 1862. Sphyrocephalus Murray, P. Z. S. p. 8.

Vom unteren Rande der Nasenlöcher verläuft eine wulstig hervortretende Hautfalte über die Oberlippe bis zum Lippenrande; weder das Männchen noch das Weibchen hat Schultertaschen. Der Schädel ist über dem letzten Molar ungefähr ebenso hoch als über dem Caninus; der vorletzte untere Molar hat aussen drei, innen einen, an der Krone gewöhnlich eingekerbten Höcker. Molaren kräftig mit deutlichen Randhöckern. Auf dem Plagiopatagium 20-28 längere Fascien, welche den vom Ellenbogen zur Spitze des Zeigefingers verlaufenden Strang durchschneiden.

Typus: Pteropus haldemani (Halow.).

Hab. West-Afrika.

1. Ep. haldemani Halowell. - 1846. Pteropus haldemani Halowell aus WestAfrika, Proc. Acad. Nat. Sc. Philad. III, Nr. 3 p. 52, Silliman's Amer. Journ. 2. ser. II, p. 273-274. - 1846. Halowell, Ann. Mag. N. H. XVIII. p. 356-357. - 1861. Hypsignathus monstrosus Allen von Alt-Calabar, Proc. Acad. Nat. Sc. Philadelphia p. 156. - 1862. Sphyrocephalus labrosus Murray von Alt-Calabar:, P. Z. S. p. 8-11, Taf. I. (Schnauze und Thier). - 1869. Fitzinger, 1. c. p. 3-4. - 1876. Ep. macrocephalus Ogilb., Peters, Sitzb. Akad. Berlin p. 475. - 1878. Ep. monstrosus Dobson 1. c. p. 6-7, 552. Taf. I. (Schnauze von ơ und 옹 ganzes Thier $\sigma^{\wedge}$ ), Taf. II., Fig. 1 (Gaumen). - 1887. Jentink, Not. Leyd. Mus. X. 49 (Lebensweise). - 1887 Jentink, Cat. Ost. p. 251. - 1888. Thomas, P. Z. S. p. 7. - 1888. Jentink l. c. p. 136 . - 1894. Matschie, Mitth. Geogr. Ges. Nat. Mus. Lübeck II. Ser. Heft 7 u. 8, p. 132-133. - 1895. Sjöstedt, Kgl. Svensk. Vet. Akad. Handl. 27 Nr. 1, p. 13, 18, 119. - 1895. Barboza du Bocage, Jorn. Scienc. Math. Phys. Lisboa 2. ser., tom. 4, XIII. p.4. - 1896. de Pousargues, Ann. Sc. Nat. Zool. III. p. 250-251. - 1897. Sjöstedt, Mitth. deutsch. Schutzgeb. X. Heft 1, p. 7. - 1897. Trouessart 1. c. p. 89. - 1897. Sjöstedt, Bihang Kgl. Svensk. Vet. Akad. Handl. 23. Afd. IV, Nr. 1 p. 11-15. - 1898. Barboza du Bocage, Jorn. Scienc. Math. Phys. Lisboa, 2. ser., tom. 5, XIX. p. 135.

Abbildungen des Schädels auf Tafel $[10]$, đa ohne sicheren Fundort $1 \mathrm{~b}, 1 \mathrm{e}$, 오 von Bibundi 1, 1a, 1c, $1 \mathrm{~d}$.

Hab. West-Afrika, im besonderen: Gambia (Dobson), Liberia (Jentink 1888), Goldküste (Jentink 1888), Togo-Land (Misahöhe, Matschie), AltCalabar (Allen und Murray), Kamerun [Bonge, Bibundi, N'dian, Itoki (Sjöstedt); Y aunde (Zenker); Victoria (Preuss)], Gabun [Dongila (Buchholz)], Ogowe, Congo français, Bangui am Ubangi (de Pousargues); Fernando Po (Barboza du Bocage), Semmio in Niam-Niam (Bohndorff bei Thomas und Stuttgarter Museum), Tingasi im Uelle-Gebiet (Thomas).

[B. M.] $\sigma^{\nearrow}$ Stevens; $2 \precsim ð, 1$ ㅇ Misahöhe, Togo, Baumann; 우 Bonge, Kamerun, Sjöstedt; ㅇ Bibundi, Kamerun, Sjöstedt; 우 Yaunde, Kamerun,

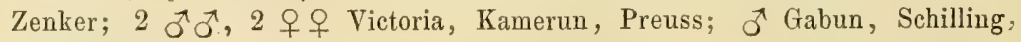
우 Dongila, Gabun, Buchholz. 


\section{Subgen: Epomophorus Bennett.}

1835. Bennett, P. Z. S. p. 149; Trans. Z. S. II. p. 31-38, Tafel 6 und 7.

Ohne Hautfalte quer über die Oberlippe; Schnauze lang; die Entfernung zwischen dem vorderen Augenrande und der Nasenspitze ist viel grösser als die Breite des Gesichtes an den Mundwinkeln. Auf dem Plagiopatagium wird der vom Ellenbogen zur Spitze des fünften Fingers verlaufende Strang von höchstens 32 langen, stark hervortretenden Fascien durchschnitten: Der Schwanz ist deutlich fühlbar. Das hintere Ende des knöchernen Gaumens verläuft nicht ungefähr in derselben Ebene wie der zwischen den Molaren befindliche Theil, sondern ist nach oben etwas aufgerollt. Der letzte obere Molar hat, falls er noch nicht zu sehr abgekaut ist, eine Ausbuchtung der Aussenkante am hinteren Ende.

Typus: Epomophorus macrocephalus Ogilb.

Hab. Afrika, südlich von der Sahara, mit Ausnahme des südwestlichen Theiles der Cap-Colonie.

Uebersicht der Arteñ :

Die Maasse gelten für ausgewachsene Thiere, bei denen die Eckzähne nicht mehr stark nach hinten gekrümmt sind, die vorderen Molaren schon Abkaunngsfächen zeigen, also nicht mehr scharfe Kanten haben, und bei denen der letzte Molar entweder ebenso hoch wie die vorderen Molaren oder aber stark abgekaut ist. Junge Thiere erkennt man auch daran, dass die Epiphysen an den Fingergelenken mit den Phalangen-Enden noch nicht verwachsen sind.

Auf dem Plagiopatagium haben die jungen Epomophorus weniger Fascien als ausgewachsene Thiere; junge Ep. neumanni und stuhlmanni, wahrscheinlich wohl auch junge wahlbergi, haben mindestens 20 Fascien, welche den Hauptstrang des. Plagiopatagiums zwischen dem Ellenbogen und der Spitze des fünften Fingers durchschneiden; bei den jungen Thieren der anderen Gruppe sind höchstens 16 solcher Fascien vorhanden. Wenn mehrere Fascien dicht neben einander verlaufen oder sich auf dem Hauptstrang schneiden, so rechne man jede Fascie einzeln.

Unterarm länger als $96 \mathrm{~mm}$. . . . . . . . Ep. büttikoferi Mitsch. p. 45

Unterarm kürzer als $96 \mathrm{~mm}$ :

Unterarm kürzer als $71 \mathrm{~mm}$ bei ô, als $64 \mathrm{~mm}$ bei ㅇ Ep. minor Dobs. p. 51

Unterarm länger als $71 \mathrm{~mm}$ bei $\widehat{o}$, als $64 \mathrm{~mm}$ bei $q$ :

Flughaut schwarzbraun oder dunkelbraun:

Flughaut schwarzbraun, hinter dem letzten Molaren zwei Gaumenfalten; Unterarm länger als $84 \mathrm{~mm}$. . . . . . . Ep. macrocephalus Ogilb. p.44

Flughaut dunkel siennabraun:

Hinter dem letzten Molaren zwei Gaumen-

falten; Unterarm kürzer als $84 \mathrm{~mm}$. Ep. anurus Heugl. p. 54

Hinter dem letzten Molaren nur eine Gaumenfalte:

Mindestens 24 Fascien auf dem Plagiopatagium:

Unterarm höchstens $81 \mathrm{~mm}$ lang Ep. neumanni Mtsch. p. 50

Unterarm mindestens $82 \mathrm{mmlang}$ Ep. wahlbergi Sund. p. 48

[Hierher vielleicht anch Ep. gamtianus Ogilb. p. 55, welcher sich von waht. bergi durch die dünne Behaarung der Füsse unterscheidet.] 
Höchstens 22 Fascien auf dem Plagiopatagium .

Ep. zenkeri Mtsch. p. 46

Flughaut sehr hellbraun oder weisslichbraun, mindestens der den Körperseiten benachbarte Theil der Flughaut weisslich:

Unterarm länger als $76 \mathrm{~mm}$ bei $\widetilde{\sigma}$, als $73 \mathrm{~mm}$ bei 우:

Höchstens 16 Fascien auf dem Plagiopatagium

[vielleicht kommt hierher auch Ep. gambianus Ogilb. p. 55, welcher sich vol angolensis durch die kürzere Schnauze und das Vorhandensein von nur einer Gaumenfalte hinter dem letzten Molaren unterscheidet.]

Mindestens 17 Fascien auf dem Plagiopatagium:

Mindestens 24 Fascien auf dem Plagiopatagium. . . . . . . Ep. stuhlmanni IItsch. p. 50

Höchstens 22 Fascien auf dem Plagiopatagium:

Unterarm kürzer als $83 \mathrm{~mm}$ Unterarm länger als $84 \mathrm{~mm}$ :

Zwei Gaumenfalten hinter dem letzten Molaren.

Eine Gaumenfalte hinter dem letzten Molaren.

Unterarm kürzer als $76 \mathrm{~mm}$ bei $\widehat{\jmath}$, als $73 \mathrm{~mm}$ bei 우.

Ep. crypturus Ptrs. p. 49

Ep. zechi Itsch. p. 46 und vielleicht $E p$. spec, vom Schari.

Ep. gambianus Ogilb. p. 55

Ep. schöensis Rüpp. p. 53

Ep. labiatus Temm. p. 54

Ep. doriae Mitsch. aus der Erythraea p. 54.

1. Ep. macrocephalus Ogilby. - 1835. Pteropus macrocephalus Ogilby vom Gambia, P. Z. S. p. 101. - 1835. Pteropus megacephalus Swainson vom Gambia, Nat. Hist. and Class. of Quadrupeds, p. 91-92, Fig. 31 (Kopf) und p. 356, Fig. 154 (Gebiss von vorn). - 1835. Pteropus epomophorus Bennett vom Gambia, P. Z. S. p. 149. - 1835. Epomophorus whitei Bennett vom Gambia, Trans. Z. S. II., p. 37-38, Taf. 6 (Thier), Taf. 7 (Haare). - 1860. Ep. macrocephalus Tomes, P. Z. S. p. 50-51. - 1861. Tomes, 1.c. Taf. I. Fig. 1, 1 a und 7 (Schädel von der Seite, der Gaumen mit den Falten und dem Gebiss, Oberkiefer). - 1869. Ep. whitei partim und Ep. macrocephalus Fitzinger 1. c. p. 10-13, 15-17. - 1870. Ep. macrocephalus. Gray, Cat. Monk., Lem. Fruit-eating Bats p. 125 ohne die Abarten. - 1878. partim Dobson 1. c. p. 8-10. 1889. Epomophorus gambianus (?) von Bolama, Barboza du Bocage, Jorn. Scienc. Math. Phys. Lisboa, 2. ser. I. p. 2-3, Fig. 2 (Gaumen mit den Falten und dem Gebiss). - 1898. Ep. macrocephalus (Ogilb.) Barboza du Bocage, l. c. V. p. 136. - (?) 1898. Ep. guineensis Barboza du Bocage von Bolama, Barboza du Bocage 1. c. p. 136.

Hab. Gambia (Ogilby), Bolama in Portugiesisch Guinea (Barboza du Bocage). Temminck hat (Esqu. Zool. p. 65-68) die von Pel an der Goldküste ge- 
sammelten Flughunde für Epomophorus whitei Benn. gehalten. Ep. whitei ist aber von Bennett (1. c. Taf. 1) mit sehr dunklen Flughäuten abgebildet worden und E. macrocephalus Ogilby vom Gambia; den die englischen Forscher mit whitei vereinigen, hat (l. c. p. 101) die Flughäute "very dark brown, nearly approaching to black". Nun sagt Temminck allerdings (p. 67): "Le système cutané est d'un brun-noirâtre [Le mâle vieux]. Les membranes sont de couleur feuille-morte [La femelle]; da aber nach Jentink.(Cat. Syst. 1888 p. 136) das Leydener Museum von der Goldküste ein ausgewachsenes Männchen dieses Flederhundes nicht besitzt, so nehme jich an, dass Temminck seine Beschreibung des alten $\sigma^{7}$ nur auf Grund der in der Litteratur vorhandenen Angaben verfasst hat. Die Epomophorus der Goldküste haben nichts mit macrocephalus Bennett zu thun, weil ihre Flughäute immer hell gefärbt sind und ihr Unterarm bedeutend länger ist als derjenige von macrocephalus. Bocage bildet den Gaumen eines Epomophorus von Bolama ab, der vielleicht hierher gehört. Er erinnert sehr an das von Tomes gegebene Bild des Gaumens von Ep. macrocephalus, besitzt aber 7 Gaumenfalten und die Zahnreihe ist nur halb so lang als die Entfernung der ersten von der 7. Gaumenfalte. Herrn Barboza du Bocage danke ich dafür, dass er mir in liebenswürdiger Weise Photographieen des Schädels seines E. macrocephalus von Bolama geschickt hat. Ich glaube, dass Ep. guineensis eine individuelle Varietät von macrocephalus darstellt.

2. Ep. büttikoferi Mtsch. spec. nov. - 1888. Epomophorus gambianus Jentink, Not. Leyd. Mus. X. p. 50. - 1888. Jentink, Cat. Syst. p. 137 c.

\section{Hab. Schieffelinsville, Junk River, Liberia.}

Bei dem. mir zu Gebote stehenden Material von Epomophoris variirt die Länge des Unterarms bei Exemplaren, deren letzter oberer Molar vollständig ausgebildet ist, nicht allzusehr. Die Männchen sind allerdings gewöhnilich grösser als die Weibchen, aber bei beiden Geschlechtern bleibt die Variation innerhalb von je 3-6 mm. Die alten Männchen von Angola erreichen eine Unterarm-Länge von $93 \mathrm{~mm}$, die kleinsten ausgewachsenen. Weibchen von dort haben einen Unterarm von $82 \mathrm{~mm}$ Länge. Die Angola-Epomophorus sind aber die grössten, welche ich aus der Gattung kenne. Der von Herrn Jentink erwähnte Epomophorus von Junk River in Liberia hat eine Unterarm-Länge von $101 \mathrm{~mm}$, also $8 \mathrm{~mm}$ mehr als der grösste sonst bekannte Epomophorus.

Die Arten von Epomophorus s. str. bewohnen beschränkte Gebiete; es ist mix nicht gelungen nachzuweisen, dass zwei Formen dieser Gruppe neben einander in derselben Gegend leben. Wo man derartige Fälle in der Litteratur findet, da darf man stets an der Richtigkeit der Fundortsangabe oder der Bestimmung zweifeln.

Bei Bolama in Portugiesisch-Guinea lebt Ep. macrocephalus, an der Goldküste eine sehr ähnliche Form, die ich jetzt als Ep. zechi abtrenne. Von Liberia ist nur die von Jentink als gambianus bezeichnete Form bekannt. Ep. gambianus stammt aber aus den. Sammlungen, welche Rendall vom Gambia heimbrachte und wird wahrscheinlich nördlich vom Gambia im Senegal-Gebiet erlangt worden sein. Es ist also von vorn herein sehr wahrscheinlich, dass in Sierra-Leone und Liberia eine neue Art von Epomophorus vorkommt. Jentink giebt die Unterarm-Länge für das eine der beiden im Leydener Museum befindlichen Stücke auf $101 \mathrm{~mm}$ an. Diagnose von Epomophorus büttikoferi Mtsch. Epomophorus, gambiano aff. sed multo major, radii longitudine $93 \mathrm{~mm}$ superante. 
Als Typus ist das von Stampfli gesammte $\widetilde{\jmath}$ des Leydener Museums von Schieffelinsville am Junk River aufzufassen. Ich habe mir erlaubt, diese Art Herrn Direktor Dr. Büttikofer in Rotterdam zu widmen, durch welchen wir die Fauna von Liberia so gut kennen gelernt haben.

Das Original-Exemplar, ein sehr altes o mit schön entwickelten Schultertaschen hat nach Jentink folgende Maasse:

\begin{tabular}{|c|c|c|c|c|c|}
\hline & arm & & & 101 & \\
\hline 3. & Finger, & Metacarpale & & 75,5 & \\
\hline 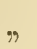 & $n$ & 1. Phalanx . & 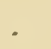 & 48 & \\
\hline & & 2. & & 64,5 & \\
\hline & & Metacarpale & & 73 & \\
\hline & 37 & 1. Phalanx . & & 35 & \\
\hline & & 2. $\quad$ & & 35 & \\
\hline & $\mathrm{rsc}$ & kel . & & 43 & \\
\hline & & Nasen & & 30 & \\
\hline
\end{tabular}

3. Ep. zechi Mtsch. spec. nov. - 1853. Pachysoma whitei partim Temminck, Esqu. Zool. Côte de Guiné p. 65-68 (Beschreibung des f p. 67 und des jungen ठ p. 68). - 1878. Ep. macrocephalus partim Dobson 1. c. p. 8-10, Taf. II. Fig. 2 (Gaumen mit Gebiss). - 1887. Ep. macrocephalus Jentink 1. c. p. 251. - 1888. Jentink 1. c. p. 136. - 1893. Matschie, Sitzb. Ges. naturf. Fr. p. 256-257. - 1894. Matschie, Mitth. Geogr. Ges. Naturh. Mus. Lübeck, 2 ser. p. 133. - 1897. partim Trouessart 1. c. p. 88.

Hab. Accra, Goldküste (Jentink), Gross-Popo, Misahöhe in Togoland (B. M.), Lagos (B. M. und Stuttgarter Museum).

Epomophorus, macrocephalo affinis, differt alis laete brunneis, radii longitudine $85-87 \mathrm{~mm}$ (우), $88-90 \mathrm{~mm}(\widetilde{ })$; dorsi colore laete brunneo griseo.

Der Epomophorus von der Goldküste, von Togo und von Lagos ist bisher immer als Ep. macrocephalus aufgefasst worden, trotzdem Ep. macrocephalus $\sigma^{\top}$ nur einen Unterarm von $86 \mathrm{~mm}$ hat und trotzdem seine Flughaut schwärzlichbraun angegeben wird. Der Epomophorus von Mittel-Guinea hat einen Unterarm von $85-87$ (ㅇ) resp. $88-90\left(\sigma^{\top}\right) \mathrm{mm}$ und seine Flughaut ist hellbraun. Der Hauptstrang des Plagiopatagiums wird von 18-22 Strängen durchschnitten. Die Entfernung der Aussenkanten der letzten oberen Molaren von einander ist beim $\widetilde{\sigma}$ wie bei macrocephalus $\sigma$ etwas länger als die Länge der oberen MIolaren-Reihe (ef. die Abbildungen bei Barboza und Tomes) mit der Abbildung auf Taf. 10. Die Zahnreihe ist länger als die Hälfte der Entfernung der ersten von der 7. Gaumenfalte. Das Bild auf Taf. II. Fig. 2 des Dobson'schen Catalogs entspricht dem Bilde, welches der Gaumen eines \& bietet. - Ich widme diese Art dem durch seine zoologischen Forschungen im. Togolande verdienten Herrn Graf $\mathrm{Zech}$.

Abbildung des Schädels eines $\widehat{\jmath}$ von Lagos auf Taf. [10], Fig. 3 als E. macrocephalus.

[B. M.] 3 o $\sigma^{\top}$ Misahöhe und Kradji, Baumann; ơ pull. Gross-Popo,

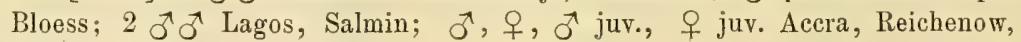
Unger und Pel.

4. Ep. zenkeri Mtsch. spec. nov. - 1865. Ep. gambianus Peters, P. Z. S. 1865 p. 400. - 1878. Ep. gambianus Dobson 1. c. p. 11 vom Gabun. - 1885. Ep. gambianus von Niam-Niam, Jentink, Not. Leyd. Mus. p. 35. - 1887. specim. b., Jentink l. c. p. 251. - 1888. specim. b., Jentink l. c. p. 137. - 1889. Ep. macro- 
cephalus und gambianus von Porto da Lenha und Netonna, Noack, Zool. Jahrb. IV. p. 200-205, Taf. V. Fig. 50-58 (Schädel von ठౌ ad. und juv., Gaumen von б’ ad. und juv.). - 1889. Ep. gambianus vom Rio Cuillo, Pungo-Andongo, Ambacca; Barboza du Bocage, Jorn. Sc. Math. Phys. Nat. Lisboa, 2. ser. I. p. 14. 1893. Ep. gambianus Matschie, Sitzb. Ges. naturf. Fr. Berlin p. 256-257. 1896. E. de Pousargues, Ann. Sc. Nat. Zool. III. p. 251-253. - 1898. partim Barboza du Bocage, Jorn. Sc. Math. Phys. Nat. Lisboa, 2. ser. V. p. 136.

Hab. Vom Kamerun-Fluss nach Süden bis zum Cuanza, nach Osten bis NiamNiam, also Süd-Kamerun, Gabun, Loango, Congo und Loanda; im besonderen bekannt vom Kamerun-Delta (Reichenow), vom Gabun (Büttner, Dobson), von Porto da Lenha (Noack), von Tschintschoscho (Falkenstein), von Netonna (Noack), von Malandje (von Mechow), von Pungo-Andongo, Ambacca, Quissango und vom Rio Cuillo (Barboza du Bocage), von Loanda (Peters), von Semmio in Niam-Niam (Jentink), von Franceville am oberen Ogowe (de Pousargues).

Dass diese Epomophorus von Nieder-Guinea nicht zu Ep. gambianus gehören, mit welchen sie in der Gestalt der Gaumenfalten grosse Aehnlichkeit haben, lässt sich aus folgenden Betrachtungen schliessen:

Bei Ep. gambianus Ogilb. variirt nach Tomes (P.Z.S. 1860 p. 531) die Länge des Unterarmes zwischen 76,2 und $81,3 \mathrm{~mm}$, bei den Exemplaren von NiederGuinea zwischen 79 und $85 \mathrm{~mm}$; es haben also die 우우 aus Nieder-Guinea einen ungefähr so langen Unterarm wie die ふ઼઼ von gambianus Ogilb. Nach der Ogilby'schen Original-Beschreibung von gambianus soll die Körperlänge $6{ }^{3} / 4$ Zoll $=171,4 \mathrm{~mm}$, die Kopflänge von der Nase zur Ohrwurzel $1 \frac{3}{4} \mathrm{Zoll}=44,45 \mathrm{~mm}$ und die Spannweite der Flügel 1 Fuss $8 \mathrm{Zoll}=508 \mathrm{~mm}$ betragen. Die Abbildung des Schädels (P. Z. S. 1861 Taf. I. Fig. 2) beweist, dass wir es mit einem alten Exemplar zu thun haben, dessen Molaren schon stark abgekaut sind. Die entsprechenden Maasse für ein altes $\sigma^{\top}$ von Tschintschoscho und ein altes + von Malandje betragen: $138 \mathrm{~mm}$; $44 \mathrm{~mm}$; ca. $555 \mathrm{~mm}$ resp. $127 \mathrm{~mm}, 43 \mathrm{~mm}$, ca. $525 \mathrm{~mm}$. Wir erkennen hieraus, dass Ep. gambianus von den Epomophorus aus Nieder-Guinea verschieden sein muss.

Von Ep.gambianus würde sich die hier zu betrachtende Art, welche ich nach meinem Freunde G. Zenker, dem ausgezeichneten Sammler in Kamerun, benenne, durch folgende Diagnose unterscheiden:

Ep. zenkeri Mtsch. spec.nov., aff. E. gambianus, differt corpore breviore, alis longioribus, radii longitudine apud 우 $78-81$, o 82 $-85 \mathrm{~mm}$.

Ob die Epomophorus aus dem Congo-Becken von solchen aus dem Gebiete des Kuanza oder solchen aus dem Ogowe- und südlichen Kamerun-Gebiet verschieden sind, muss eine Vergleichung reicheren Materials lehren. Dobson's Fig. 3 Taf. II. könnte hierher gehören.

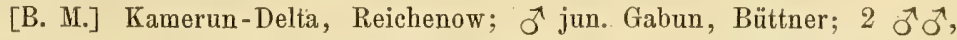

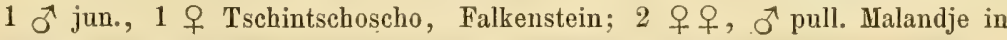
Angola, von Mechow; + juv. Angola, Lissaboner Museum.

5. Ep. angolensis Gray. - 1870. Ep. macrocephalus var. angolensis Gray von Angola, Cat. Monk. Lem. Fruit-eating Bats. p. 125. - 1870. Ep. macrocephalus Peters von Caconda und Benguella, Jorn. Sc. Lisboa III. Nr. X. p. 123. 1872. Ep. macrocephalus Peters, P.Z.S. p. 364. - 1878. Ep. gambianus partim 
Dobson I. c. p. 10-11. - 1882. Ep. gambianus Barboza du Bocage; Jorn. Sc. Lisboa IX. p. 25. - 1888. Jentink 1. c. p. 137. - 1889. Ep. gambianus von Benguella und Caconda, Barboza du Bocage, Jorn. Sc. Lisboa I. p. 2 und 14. - 1898. Bocage 1. c. V. p. 136.

Hab. Angola, südlich vom Kuanza bis zum Tsoakhaub-Fluss in DeutschSüdwest-Afrika, im besonderen: Caconda, Benguella (Bocage), Koelae ei KasingaFluss in Mossamedes (Jentink), Tsoakhaub-Fluss (Higgins im B. M.).

Gray giebt für seinen $E_{p}$ : angolensis folgende Diagnose:

Pale brown; head with a dark streak from the nostrils to the front of the lower edge of the eye, and a second rather above and parallel with the upper lip; chest and beneath whitish; epaulette pale brown; forearm $3^{1 / 4}$ inches. Female. Hab. Angola, Monteiro (in spirit).

Unsere 4 Exemplare, die ebenfalls von Monteiro gesammelt sind, haben; wie es scheint, keine Kopfzeichnung; sie sind aber anch unten weisslich und zeichnen sich durch besondere Grösse aus.

Ep. angolensis hat in der Bildung des Gaumens und in der Stellung der Gaumenfalten grosse Aehnlichkeit mit dem Ep. macrocephalus, wie ihn Tomes (P. Z. S. 1861 Taf. II.) abbildet, die fünfte Querfalte ist aber nicht durchlocht, sondern getheilt oder wenigstens stark gefurcht. Die Flughäute sind sehr hell, weisslichgrau. Auf dem Plagiopatagium durchschneiden höchstens 16 Stränge den Hauptstrang; der Unterarm ist bei ㅇ mindestens $82 \mathrm{~mm}$, bei of mindestens $89 \mathrm{~mm}$ lang, wenn sie das vollständige Gebiss haben.

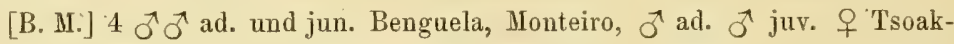
haub-Fluss, Walfisch-Bay, Higgins, ô juv. Caconda, Lissaboner Museum.

\section{Ep. spec. nov.?}

Unter Nr. 4240 haben wir im Berliner Museum ein $q$ in Alcohol, welches zusammen mit zwei Stücken von angolensis aus den Monteiro'schen Sammlungen stammt. Es unterscheidet, sich von diesen aber dadurch, dass die Flughäute dunnkel sind, dass ca. 25 Stränge auf dem Plagiopatagium den Hauptstrang durchschneiden und dass der Unterarm nur $77 \mathrm{~mm}$ lang ist. Durch diese Merkmale steht es am nächsten den Exemplaren aus Ukambani in Deutsch-Ost-Afrika, unterscheidet sich aber von diesen ungefähr gleich alten Mombassa-Weibchen durch etwas kürzeren Unterarm (77 mm gegen $78 \mathrm{~mm}$ ), durch viel längere Ohren: $24,5 \mathrm{~mm}$ gegen $21 \mathrm{~mm}$ und durch längeren Kopf (52 mm gegen $50 \mathrm{~mm})$.

Es kann dieses Stück. nur aus dem Okawango-Quellländern im südlichen Angola stammen und würde dann wohl den Epomophorus des Salzpfannen-Gebietes (Okawango, Ngami, Botletle) darstellen.

[B. M.] 우 Angola, Monteiro.

Vom Orangefluss-Gebiet kennen wir noch keinen Epomophorus, ebenso wenig ist ein solcher aus dem südwestlichen Caplande bisher bekannt geworden.

\%. Ep. wahlbergi Sundevall. - 1846. Epomophorus wahlbergi Sundevall von Port Natal und Inner-Kaffraria, Sundevall, Oefv. Vet. Akad. Stockholm p. 118. - 1861. Ep. crypturus Tomes, P. Z. S. p. 11-12. - 1869. Fitzinger 1. c. p. 13-14. - 1878. Dobson 1. c. p. 11. - 1887. Ep. gambianus von Port Natal, Jentink p. 251. - 1888. Jentink von Port Natal, 1. c. p. 137.

Hab. Port Elisabeth bis Port Natal. 
Charakteristisch für diese Art scheint die dichte Behaarung zu sein, welche auch die Schenkel und einen Theil der Flughäute bedeckt. Der Unterarm ist bei unseren o $85-86$, bei dem \& $84 \mathrm{~mm}$ lang, auf dem Plagiopatagium verlaufen 24-26 Stränge durch den Hauptstrang; die Flughaut ist dunkelbraun und merkwürdig fest, wenig durchsichtig. Tomes hat (P.Z.S. 1861 p.11-12) als Pt. crypturus diese Art beschrieben; er erwähnt ebenfalls die dichte Behaarung des Körpers, eines Theils der Flughäute, namentlich an dem Unterarm, den Schenkeln und Füssen, sagt, dass die Ohren breiter aber nicht länger als bei gambianus wären und dass die Interfemoral-Membran namentlich am Calcaneum breiter sei als bei gambianus.

Oldf. Thomas erwähnt, dass Ep. crypturus für Natal durch Shelley 1881 nachgewiesen sei. Es wäre dieses Vorkommen ausserordentlich interessant.

Mir ist in allen den Fällen, wo mehrere Abarten der Untergattung Epomophorus in einem und demselben faunistischen Untergebiet neben einander vorkommen sollten, soweit sie Exemplare unserer. Sammlung betrafen, stets der Nachweis gelungen, dass durch ungenaue Etiquettirung oder falsche Bestimmung Verwirrung: verursacht war.

Ich habe bis jetzt noch nicht den Beweis dafür erhalten, dass 2 Abarten dieser Gruppe das gleiche Vaterland bewohnen.

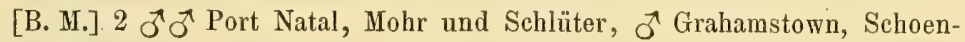
land, $q$ Durban, Hahn.

Aus dem Gebiet des Limpopo ist ein Epomophorus noch nicht bekannt.

8. Ep. crypturus Ptrs. - 1852. Epomophorus crypturus Peters von Tette, Reise Mossamb. Säugethiere p. 26-31, Taf. V. (Thier, Schwanzflughaut und Kopf), Taf. XIII., Fig. 1-6 (Schädel). - 1852. Peters, Monatsb. Akad. Berlin p. 757. - 1869. Fitzinger l. c. p. 17-19. - 1870. Ep. macrocephalus var. unicolor Gray von Shupanga am Zambese, Cat. Monk. Lem. Fruit-eating Bats. p. 125. - 1878. Ep. gambianus Dobson 1. c. p. 11. - 1889.-Ep. crypturus Barboza du Bocage, Jorn. Sc. Lisboa I. p. 3-4. - 1894. Thomas, P. Z. S. p. 137-138. 1898. Barboza du Bocage, Jorn. Sc. Lisboa V. p. 136-137.

Hab. Tette, Zomba und Shupanga im Gebiet des Zambese.

Ep. unicolor Gray gehört wohl hierher.

Ich habe noch im Jahre 1894. (Mitth. Lübeck p. 133) behauptet, „Peters habe sowohl Exemplare von gambianus als von crypturus gesammelt und den Unterschied beider Arten nicht beachtet". Ich wurde zu dieser Behauptung dadurch verleitet, dass ich zwei Epomophorus mit Peters Namen etiquettirt fand, welche nicht zu crypturus gehörten. Diese sind aber, wie sich jetzt herausgestellt hat, auf dem Schreibtisch von Prof. Peters nach dessen Tode aufgefunden worden und haben die vorläufige Bemerkung "Peters" auf dem Etiquett erhalten. Es waren' Mombas-Flederhunde, die von Hildebrandt gesammelt worden sind. Für das Vorhandensein eines zweiten Epomophorus im Zambese-Gebiet ist der Beweis noch nicht erbracht worden.

Bei Ep. crypturus ist der Unterarm des + 78-79; das noch nicht ansgewachsene $\widehat{\sigma}$ unseres Museums hat einen Unterarm von $77 \mathrm{~mm}$ Länge. In der Gestalt der Gaumenfalten ist Ep. crypturus ähnlich Ep. macrocephalus, anurus und zechi, von kleineren Arten, deren Gaumenbeschaffenheit wir kennen, Ep. minor.

Abbildung des Schädels eines $\$$ von Tette auf Taf. [11], Fig. 3a-e. [B. M.]. ơ juv., 2 우오, 2 우 오 juv., Tette, Peters. 
9. Ep. stuhlmanni Mtsch. spec. nov. - 1878. Ep. gambianus von Dar es Salaam. Dobson l. c. p. 11. - 1890. (?) Ep. minor von Bagamoyo, Thomas, P. Z. S. p. 446. - 1891. Ep. gambianus Noack, Jahrb. Hamb. Wiss. Anst. IX. p. 57-58. - 1891. Ep. minor Noack, 1. c. p. 58-59. - 1895 Ep. gambianus Matschie, Säugethiere Deutsch-Ost-Afrikas, p. 16 Fig. 7 (Kopf). - 1897. Matschie, Arch. Naturg. I. p. 83.

\section{Hab. Küstengebiet von Deutsch-0st-Afrika und Zanzibar.}

Diese Abart hat ebenso wie wahlbergi und wie die von Mombas bekannten Flederhunde auf dem Plagiopatagium mehr als 23 Stränge, welche den Hauptstrang durchschneiden. Von wahlbergi und dem Mombas-Flederhund lässt sie sich leicht durch ihre gegen die Körperseiten weisslich überflogenen Flughäute unterscheiden; sie ist grösser als der Mombas-Flederhund, aber kleiner als wahlbergi. Ihre Diagnose könnte man in dem Satze zusammen fassen:

Epomophorus stuhlmanni Mtsch. spec. nov., alis ad corporis latera albido tinctis, fasciis in plagiopatagio $23-30$; radii longitudo $85-87 \mathrm{~mm}\left(\sigma^{\top}\right), 80-81 \mathrm{~mm}$ (ㅇ).

In der Gaumenbildung steht diese Abart der Kamerun-Form sehr nahe und dem „gambianus" auf Dobson's Tafel. Noack's Ep. minor von Zanzibar sind junge Ep. stuhlmanni; ich möchte auch vermuthen, dass die Ep. minor, welche Oldf. Thomas von Bagamoyo aufführt, junge Ep. stuhlmanni sind. Die grössten Ep.

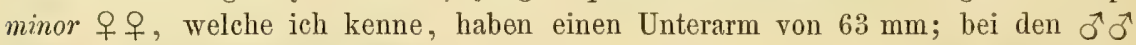
ist der Unterarm 64-68 mm lang; ganz junge Ep. stuhlmanni haben schon eine Unterarm-Länge von $68-69 \mathrm{~mm}$.

Ich nenne diese Abart nach Herrn Regierungsrath Dr. Stuhlmann, dem ich den grösseren Theil der mir zur Verfügung stehenden Exemplare verdanke.

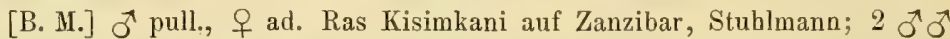
Mojoni auf Zanzibar, 0. Neumann; 2 우우 pull., 3 우우, 1 저 Dar es Salaam,

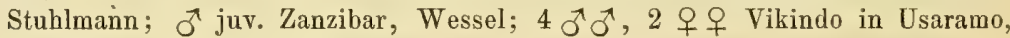
Stuhlmann; ㅇ Lindi, Fülleborn.

10. Ep. neumammi Mtsch. spec. nov. - 1876. Ep. crypturus Ptrs. Monatsb. Akad. Berlin p. 913. - 1878. Peters 1. c. p. 195. - 1879. Ep. labiatus Peters 1. c. p. 831.

Epomophorus neumanni Mtsch. spec. nov. alis concoloribus neque ad corpis latera albido tinctis, fasciis in plagiopatagio $23-31$; radii

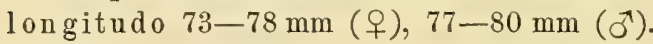

\section{Hab. Küstengebiet von Britisch Ost-Afrika (Mombasa, Takaungu, Malindi)} und Moschi am Kilima-Ndjaro.

Ep. neumanni, den ich Herrn Oscar Neumann widme, unterscheidet sich von allen andern Flederhunden der Untergattung Epomophorus mit Ausnahme von Ep. wahlbergi und stuhlmanni dadurch, dass auf dem Plagiopatagium mindestens 23 Stränge den Hauptstrang durchschneiden; junge Thiere zeigen weniger Stränge, aber immerhin über 20, während junge Exemplare der übrigen Arten höchstens 16 aufweisen. Von Ep. wahlbergi und stuhlmanni unterscheidet sich Ep. neumanni durch die weit geringere Grösse, von stuhlmanni auch durch die gleichmässig braun gefärbten, an den Körperseiten nicht weisslichen Flughüute. Die Gaumenfalten sind ungefähr so, wie sie Dobson für gambianus abbildet. Ep. neumánni sieht 
Ep. zenkeri von Kamerun sehr ähnlich, ist aber kleiner und hat zahlreichere Stränge auf der Flughaut.

[B. M.] $2 \sigma^{\nearrow}, 1$ \& Mombasa, Hildebrandt; $\sigma^{\Uparrow}$ jun. Malindi, Fischer; ㅇ jun. Moschi am Kilima-Ndscharo, Oscar Neumann; ot, ㅇ Takaungu, Thomas.

11. Ep. minor Dobs. - 1879. Epomophorus minor Dobson aus der Sammlung des Herrn Dr. Robb, Dobson, P. Z. S. p. 715-716. - 1880. Dobson, Report Brit. Ass. p. 2. - 1887. von Gonda in Ugunda, Deutsch-Ost-Afrika, Noack, Zool. Jahrb. p. 268-271, Taf. X, Fig. 28-30 (Schädel).

Hab. Gebiet des Malagarasi: Gonda in Ugunda, Ujiji am Tanganyika.

Ep. minor unterscheidet sich durch seine geringe Grösse leicht von allen anderen Abarten; der Unterarm des $q$ ist nicht länger als $63 \mathrm{~mm}$, derjenige des $\widetilde{\sigma}$ 63,5-68 mm lang. Bei Ep. minor ist der Gaumen ungefähr so gestaltet, wie ihn Dobson für macrocephalus abbildet.

Dobson hat die Original-Exemplare von Herrn Dr. Robb in Zanzibar erhalten. Thomas erwähnt Ep. minor ausserdem von Bagomoyo und Kiriamo (P. Z. S. 1890 p. 446) und von Turquell im Sük-Laride (P. Z. S. p. 182), Noack nennt ihn von Zanzibar (Zool. Jahrb. Hamb. Wiss. Anst. 1891 p. 58-59), Jentink (Cat Mamm. 1888 p. 136) von Schoa.

Der Fundort eines Exemplares, welches Fischer angeblich bei Bagamoyo gesammelt haben soll, ist, wie eine genaue Untersuchung ergeben hat, nicht ganz sicher. Fischer hatte in der Sendung, welche damals nach Berlin kam, eine Anzahl von Säugethieren geschickt, welche von seinen Jägern sowohl an der ZanzibarKüste, als aus dem Innern sowie im Somali- und Gallalande gesammelt worden waren. Es ist möglich, dass das Exemplar von Bagamoyo kam, es ist aber ebenso gut möglich, dass es irgendwo anders gefangen ist. Eine genaue Etiquette trug das Thier nicht.

Noack's Ep. minor von Zanzibar ist Ep. gambianus juv.

Ob Dr. Robb die Ep. minor wirklich auf Zanzibar gesammelt hat, geht aus Dobson's erster Mittheilung nicht mit Sicherheit hervor; jedenfalls hat Robb auch Ep. labiatus von Abessynien gehabt.

Böhm's Stücke sind zum grösseren Theile trockene Bälge; wir besitzen aber ausserdem ein $q$ in Alcohol aus Böhm's Sammlungen, welches leider seiner Zeit, ebenso wie alle übrigen von Böhm gesammelten Alcohol-Exemplare, Herm Professor Dr. Noack zur wissenschaftlichen Bearbeitung nicht zugänglich gemacht worden ist. Mit diesem $f$ stimmt ein $f$ aus Robb's Sammlung, welches wir besitzen, in der Grösse, der Bildung des Gaumens, der Anzahl der PlagiopatagialStränge, der Länge der Molarenreihe ziemlich gut überein, nur die fünfte Gaumenfalte ist nicht so deutlich durchlocht wie bei den beiden anderen AlcoholExemplaren, sondern in der Mitte nur durch eine tiefe Furche getheilt. Sonst aber ist die Lage der einzelnen Falten bei allen drei Stücken die gleiche.

Ein ơ und ein $q$ juv. von Ujiji am Tanganyika, welche Dr. Hösemann dem Museum schenkte, stimmen hinsichtlich der Gaumenfalten vollständig zu dem Exemplare aus der Robb'schen Sammlung.

Dieses $ð$, ein ausgewachsenes, altes Exemplar von $68,5 \mathrm{~mm}$ Unterarmlänge, hat keinerlei auffallende Behaarung an den Schultertaschen; es ist am 17. XI. erlegt. Dagegen zeichnet sich ein anderes ơ mit nur $64 \mathrm{~mm}$ Unterarmlänge durch schön entwickelte Epauletten aus. Leider steht der genaue Fundort dieses letzteren Stückes nicht fest; ich kenne auch voriäufig den Sammler noch nicht, schliesse aber 
aus gewissen Anzeichen, dass dieses Exemplar aus den Quellgebieten der Malagarasi-Zuflüsse im östlichen Ukonongo, Deutsch-Ost-Afrika, stammt.

Ich bin der Ueberzeugung, dass die kleinen Epomophorus aus dem Malagarasi-Gebiet als E. minor Dobson bezeichnet werden müssen.

Thomas erwähnt Ep. minor für Bagamoyo; wenn diese Bestimmung richtig ist, so wäre dadurch der Beweis geführt, dass Ep. minor entweder zu gewissen Jahreszeiten seine Heimath verlässt und in andere Gebiete einwandert, oder dass Ep. minor neben einer anderen Art der Epomophorus-Gruppe in dem gleichen Gebiete lebt. Jedenfalls ist eine Nachprüfung der Bestimmung dieses Stückes sehr interessant.

Im Berliner Museum befindet sich ein von Herrn Dr. Stuhlmann bei Kirimba an der Nordwestecke des Albert-Edward-See's am 18. I. 92 gesammeltér Balg eines kleinen Epomophorus, welcher sicher nicht zu Ep. minor gehört. Es ist ein $q$ mit sehr stark entwickelten Zitzen, der Unterarm hat eine Länge von $66 \mathrm{~mm}$; auf der Bauchmitte befindet sich ein länglicher, von den graubraunen Bauchseiten scharf abgesetzter weisser Fleck. Der Schädel ist viel länger als derjenige eines alten 우 von Ep. minor [38 gegen $34 \mathrm{~mm}$ Basallänge], auch die Molarenreihe ist länger [9,1 mm gegen $8,4 \mathrm{~mm}]$.

Dieser Flughund stimmt in allen seinen Merkmalen viel besser zu einem Exemplare, welches wir von Gerrard gekauft haben und welches aus Bogos stammen soll; nur hat dieses Stück, ebenfalls ein altes 우, einen bedeutend längeren Unterarm $(69,5 \mathrm{~mm})$.

Aus Nordost-Afrika sind 3 Epomophorus beschrieben, welche bei der Bestimmung unserer Stücke zunächst in Frage kommen. Ep. anurus Heugl. hat einen Unterarm von $74,5-81,5 \mathrm{~mm}$ Länge, kommt also nicht in Betracht. Für eines der Original-Exemplare von Ep. labiatus, das von Temminck beschriebene o giebt Tomes (P. Z. S. 1861 p. 11) eine Unterarm-Länge von $63,5 \mathrm{~mm}$ an, die mit Temminck's Angaben ungefähr übereinstimmt. Dobson erwähnt, dass dieses ơ noch jung ist (P. Z. S. 1879 p. 716) und dass im Leydener Museum noch ein altes + existire, welches offenbar zu derselben Art gehöre. - Endlich hat Rüppel nach einem sehr jungen Exemplar einen Epomophorus schoensis beschrieben.

Herr Professor Dr. Lampert, Direktor des Königl. Naturalien-Cabinets in Stuttgart, hat die Liebenswürdigkeit gehabt, mir die Original-Exemplare der von Heuglin gesammelten Flughunde gütigst zur Untersuchung nach Berlin zu senden. Unter den mir nunmehr vorliegenden Stücken befindet sich auch ein altes 우 eines Epomophorus aus dem Bellegas-Thale, also nördlich vom Tana-See und westlich vom Siemen-Gebirge aus demselben Gebiete, aus welchem E. labiatus Temm. beschrieben worden ist. Der Unterarm dieses $q$ ist $67,5 \mathrm{~mm}$ lang. Der Schädel. ist länger als derjenige des $q$ von Kirimba (40 mm Basallänge gegen $38 \mathrm{~mm}$; $10 \mathrm{~mm}$ Länge der oberen Molarenreihe gegen 9,1 mm) und die Molaren sind bedentend breiter (Breite des zweiten Praemolaren bei labiatus $1,8 \mathrm{~mm}$, bei dem 우 von Kirimba $1,5 \mathrm{~mm}$ ). Der Bellegas-Schädel stimmt nun vollständig überein mit einem Schädel, welcher sich in den Sammlungen des Reisenden Schiller befand, die in der alten Anatomischen Sammlung aufbewahrt wurden und jetzt dem Kgl. Museum für Naturkunde gehören. Der genaue Fundort war nicht mehr zu ermitteln, jedenfalls aber stammt der Schädel aus dem mittleren Abessynien.

Ich halte den Beyveis für erbracht, dass der Kirimba-Epomophorus weder zụ Ep. minor noch zu Ep. labiatus gestellt werden darf. 
Ob der Kirimba-Epomophorus einer noch unbeschriebenen Abart angehört oder zu schoensis Rüpp. zu rechnen ist, das wage ich vorläufig nicht zu entscheiden. Ich vermuthe auch, dass der von Kiriamo durch Thomas erwähnte Epomophorus ebenfalls nicht zu Ep. minor gestellt werden darf.

Ich halte ich es für wahrscheinlich, dass auch in Nordost-Afrika genau so wie in den südlichen Gegenden die Epomophorus zur Bildung von geographischen Abarten neigen, und dass man vielleicht in jedem faunistischen Untergebïet je eine Abart finden wird.

Ep. labiatus ist von Botta im Sennaar entdeckt worden, bewohnt also wahrscheinlich das ganze Gebiet des Atbara und des Bahr el Abiad. Bei ihm ist der Unterarm zwischen 63,5 und $67,5 \mathrm{~mm}$ lang nach den Angaben von Temminck, Tomes und Heuglin.

Südlich von diesem Gebiet, vielleicht vom Tana-See und von Siemen nach Süden, würde $E p$. schoensis zu suchen sein.

Wenn wir uns erinnern, dass z. B. Papio doguera, Colobus guereza und Equus grevyi sowohl in Schoa als im Gebiet des Rudolf-See's gefunden worden sind, so wird es wahrscheinlich, dass der von Turquell erwähnte, ebenso wie der für Schoa angegebene Epomophorus zu El . schoensis Rüpp. gehören und dass das Verbreitungsgebiet von Ep. schoensis sich bis in die Nähe der Nordostecke des Nyansa erstreckt, ja vielleicht in dem eigenthümlichen Mischgebiet der Uferländer des Victoria-Nyansa, wo die Urwälder rein westliche Thierformen, die Steppen östliche Arten beherbergen, bis zum Albert-Edward-See sich ausdehnt.

Wir wissen noch nicht, welche Abart des Epomophorus das Eyassi- und MassaiGebiet bewohnt, welche auf dem. Somali-Plateau zu finden ist und welche die nördliche Somali-Küste bewohnt.

Abbildung des Schädels eines of von Goṇda auf Tafel [11], Fig. $2 \mathrm{a}-\mathrm{e}$.

[B. M.] 우 angeblich Bagamoyo, Fischer; 우 angeblich Zanzibar, Robb;

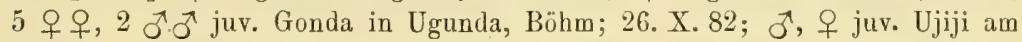
Tanganyika, 17. XI. 97 Hösemann; $\sigma^{\widehat{T}}$ mit fraglichem Fundorte, vielleicht aus Ost-Ukonongo.

12. Ep. schoensis Rüpp. - 1842. Pteropus schoënsis (Rüppell) von Schoa, Mus. Senckenb. III. p. 131-132. - 1867. Peters, Monatsb. Akad. Berlin p. 869. 1869. Fitzinger, Sitzb. Akad. Wien LX. 1. Abth. p. 19-21. - 1877. Pteropus (Epomophorus) schovanus v. Heuglin, Reise N.-O.-Afrika II. p. 18. - 1878. Ep. labiatus partim Dobson 1. c. p. 11. - 1887. Ep. minor von Let Marefia und Farré, Monticelli, An. Mus. Civ. St. Nat. Genova, 2. ser. (V) p. 523. 1888. Ep. minor von Schoa, Jentink 1. c. p. 136. - 1890. (?) Ep. minor von Kiriamo, Thomas, P. Z. S. p. 446. - 1891. Ep. minor von Turquell, Thomas, P. Z. S. p. 182.

Hab. Schoa und wahrscheinlich das Gebiet des Rudolf- und Stephanie-See's, vielleicht nach Westen bis zum Albert- und Albert-Edward-See.

Hierher gehören vermuthlich die Stücke des Britischen Museums von Kiriamo und Turquell, das Leydener Exemplar von Schoa, die Thiere im Museum von Genua von Let Marefia (Antinori coll.), Farre in Schoa (Ragazzi coll.) und Guma (Traversi coll:).

[B. M.] 오 ad. Kirimba an dem Nordwestufer des Albert-Edward-See's, Stuhlmann, 18. I. 92. 
13. Ep. labiatus Temminck. - 1837. Pteropus labiatus von A byssinien, Temminck, Monogr. Mamm. II. p. 83-84, Taf. 39, Fig. 2 und 3 (Kopf des $\sigma^{7}$ und ㅇ). 1842. Pteropus whitei Benn. von Sennar, Rüppell, Verz. Senck. Samml. p. 10. - 1869. Fitzinger 1. c. p. 8-9. - 1877. v. Heuglin, Reise N.-0.-Afrika II. p. 15-16. - 1878. Dobson 1. c. p. 11. - 1887. Jentink 1. c. p. 251. 1888. Jentink l. c. p. 137. - 1897. Trouessart l. c. p. 88.

Hab. Gebiet des Atbara und Bahr el Abiad.

14. Ep. doriae Mtsch. spec. nov. - 1879. (?) Ep. labiatus Dobson, P. Z. S. p. 716 -717. - 1880. (?) Dobson, Rep. Brit. Ass. p. 2-3. - 1887. Ep. labiatus von Keren, Bogos, Monticelli, An: Mus. Civ. St. Nat. Genova, 2. ser. (V) p. 524.

Epomophorus doriae Mtsch. spec. nov. aff. Ep. minor, molaribus angustis, $1,5 \mathrm{~mm}$ latis, differt radii longitudine apud $q 69,5 \mathrm{~mm}$.

Ich verknüpfe mit diesem Flederhund den Namen des Herrn Marquis Doria, welchem unsere Sammlung so ausserordentlich grosse Förderung zu danken hat.

E. doriae unterscheidet sich von Ep. labiatus durch seine schmalen Backenzähne, welche nur 1,5 mm breit sind und durch die kurze Molarenreihe (9,2 mm); er unterscheidet sich von minor durch den längeren Unterarm $(69,5 \mathrm{~mm}$ bei $q$ ) und durch die längere Schnauze (15,9 mm vom vordersten Punkte des Augenrandes neben dem Foramen infraorbitale bis zum Gnathion - gegen 14,1 bei einem E. minor-Weibchen).

Wie sich Ep. doriae zu Ep. schoensis verhält, vermag ich vorläufig nicht zu sagen. Von dem Kirimba-Flederhunde, mit welchem er in der Länge der Zahnreihe und der Gestalt der Molaren übereinstimmt, unterscheidet er sich durch die grössere Länge des Unterarmes und dadurch, dass bei doriae der -erste untere Molar viel länger ist als der letzte Praemolar, während bei dem Kirimba-Flederhund beide Zähne ungefähr gleich lang sind.

Hab. Küste des Rothen Meeres in der Erythraea.

Ich möchte vermuthen, dass die Ep. labiatus aus der Robb'schen Sammlung hierher gehören, ebenso wie die Exemplare von Bogos (Beccari) im Museum von Genua.

[B. M.] 우 Bogos, Gerrard.

15. Ep. anurus Heugl. - 1864. Pteropus (Epomophorus) anurus von Bongo, Central-Afrika, v. Heuglin, Nov. Act. Acad. Carol. Leop. Nat. Curios V., XXXI. p. 12-13. - 1869. Fitzinger, 1. c. p. 5-7. - 1877, v. Heuglin, Reise N.-O.-Afr. p. 16-17. - 1878. Ep. labiatus partim Dobson 1. c. p. 11. - 1897. Trouessart l. c. p. 88 .

Hab. Bongo, Gebiet des Bahr el Gazal, Central-Afrika.

Zu den Beschreibungen von Heuglin kann ich hinzufïgen, dass der Unterarm des 우 $81,5 \mathrm{~mm}$, derjenige des 우 $74,5 \mathrm{~mm}$ misst, dass auf dem Plagiopatagium weniger als 22 Fascien den Hauptstrang vom Ellenbogen zur Spitze des fünften Fingers durchschneiden, und dass Ep. anurus von Ep. crypturus sich durch die dunkelbraunen Flughäute und die scharf abgesetzte schneeweisse Bauchmitte unterscheidet. Die Schädel beider Abarten sind sehr ähnlich.

Ich habe Herrn Professor Dr. Lampert für die mir liebenswürdigerweise ermöglichte Untersuchung der Original-Exemplare dieser Art zu danken. 
16. Ep. spec. nov.? - 1896. Ep. macrocephalus aus der Gegend zwischen Y abanda und Mpoko, $7^{\circ}$ n. Br., $17^{\circ} 50$ östl. L., E. de Pousargues Ann. Sc. Nat. Zool. III. p. $253-254$.

"Les dimensions de cet individu dépassent celles que Dobson assigne à la femelle de cette espèce, et atteignent presque les proportions du mâle. Mais à part cette légère différence, il n'y a pas à se méprendre aux caractères fournis par la longueur et l'étroitesse du museau, la forte saillie et la divergence des narines et surtout par le nombre et la disposition des replis du palais, dont le 5e très renflé, présente à son centre la depression losangique, signe critérium de l'espèce." So lautet die Beschreibung, welche E. de Pousargues gegeben hat. Der Fundort des Exemplars liegt im Schari-Gebiet, nordwestlich vom Knick des Ubangi. Wir besitzen durch die Herren Zenker und von Carnap-Quernheimb zwei ganz junge Epomophorus aus dem. Hinterlande von Kamerun, wahrscheinlich aus dem östlichen Wute oder aus der Nähe von Ngaundere (genauere Angaben habe ich von den betreffenden Herren, die beide angenblicklich in Afrika weilen, erbeten). Diese Thiere, beides $\widehat{\jmath} \widetilde{\jmath}$, haben noch das Milchgebiss, aber die definitiven Zähne sind schon sichtbar.

Vergleicht man diese wahrscheinlich aus dem Logone-Gebiet, also einem Theile des Schari-Gebietes stammenden Flederhunde mit einem noch etwas jüngeren ơ von Klein-Popo im Togolande, so ergiebt sich folgendes:

Die Flughaut ist bei beiden hellbraun, weisslich überflogen. Der Togo-Flederhund hat einen viel grösseren Kopf als die beiden Kamerun-Exemplare; bei den letzteren sind aber die Unterarme beträchtlich länger als bei dem Togo-Stück. Nach den Angaben des Herrn de Pousargues würde sein Exemplar, ein $q$, auch grösser sein wie ein 우 von Mittel-Guinea. Ich bin davon überzeugt, dass der Schari-Epomophorus als Abart von Ep. macrocephalus zu trennen ist, obwohl er, wie anch unsere jungen Thiere zeigen, in der Gestalt der Gaumenfalten ihm sehr ähnlich ist. Da ich aber ein erwachsenes Thier nicht habe untersuchen können, so beschränke ich mich darauf, die Wahrscheinlichkeit anzudeuten, dass im Tschadsee- und Schari-Gebiet ein Epomophorus, der von Ep. macrocephalus verschieden ist, lebt.

\section{Hab. Tschad-See-Gebiet.?}

[B. M.] 2 ठ઼ pull., Hinterland von-Kamerun, Zenker und von Carnap. Aus dem Gebiet des oberen Niger kennen wir einen Epomophorus noch nicht.

17. Ep. gambianus Ogilb. - 1835. Pteropus gambianus Ogilby aus der Rendall'schen Sammlung vom Gambia, P. Z. S. p. 100-101. - 1860. Tomes, P. Z. S. p. 52-53. - 1861. Tomes, P. Z. S. Taf. I., Fig. 2, $2 \mathrm{a}$ und $2 \mathrm{~b}$ (Schädel). 1869. Fitzinger 1. c. p. 1-3. - 1878. Ep. gambianus partim, Dobson, 1. c. p. 10-11, Taf. II., Fig. 3 (Kopf) und 3a (Gaumen). - 1897. Ep. gambianus partim, Trouessart 1. c. p. 88.

Hab. Nicht festgestellt. Vom Gambia wurden die Original-Exemplare nach London gebracht.

Meiner Ansicht nach könnte das Senegal-Gebiet das Vaterland von Ep. gambianus sein. Es ist nicht wahrscheinlich, dass zwei verschiedene Arten von Epomophorus s. str. in demselben Faunen-Gebiete leben. Vom Gambia-Becken kenuen wir Ep. macrocephalus, von Liberia Ep. büttikoferi; aus dem Senegal-Gebiet ist aber ein Epomophorus noch nicht beschrieben. Es ist deshalb möglich, dass Ep. 
gambianus im nördlichen Senegambien den gambianischen Ep. macrocephalus ersetzt.

Abbildung des Schädels eines wahrscheinlich hierher gehörigen Exemplares ohne genauen Fundort auf Taf. [10], Fig. 2a-c.

[B. M.] $\sigma^{\nearrow}$ ohne genauen Fundort. Gerrard.

\section{Subgen. Epomops Gray.}

1870. Gray Cát. Monk. Lem. Fruit-eating Bats p. 126.

Ohne Hautfalte quer über die Oberlippe. Schnauze kurz; die Entfernung zwischen dem vorderen Augenwinkel und der Nasenspitze ist ungefähr so breit, wie das Gesicht an den Mundwinkeln. Auf dem Plagiopatagium wird der vom Ellenbogen zur Spitze des fünften Fingers verlaufende Strang von mindestens 36 langen, stark hervortretenden Fascien durchschnitten. Der Schwanz ist nicht zu fühlen. Das hintere Ende des knöchernen Gaumens verläuft ungefähr in derselben Ebene wie der zwischen den Molaren befindliche Theil desselben. Der letzte obere Molar ist nach hinten gleichmässig verschmälert.

Typus: Epomops franqueti Gray.

Hab. West-Afrika, Ober- und Nieder-Guinea, Congo-Gebiet, Seeen-Gebiet.

\section{Uebersicht der Arten:}

Die dritte Gaumenfalte an den mittleren Molaren ist ungetheilt Ep. comptus Allen p. 57

Die dritte Gaumenfalte an den mittleren Molaren ist mit der

zweiten verschmolzen und entsendet je einen Arm zum

ersten Praemolaren und Molaren . . . . . . . . Ep. dobsoni Boc. p. 57

Die dritte Gaumenfalte an den mittleren Molaren ist ersetzt

durch eine grosse ovale Papille jederseits . . . . Ep. franqueti Tomes p. 56

Ep. dobsoni kenne ich nur aus der Litteratur. Von Ep. franqueti haben wir

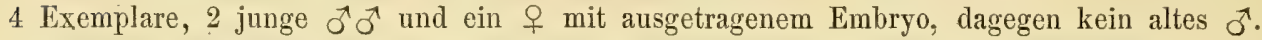
Ich vermag deswegen noch nicht die Unterschiede anzugeben, welche zwischen diesen Arten ausser der Gestalt der Gaumenfalten bestehen. Ein ㅇ vom oberen Volta, welches die für franqueti charakteristischen Gaumenfalten besitzt, ist viel kleiner als das einzige 우 in Alcohol, welches wir von Ep. comptus haben und welches vom Gabun stammt. Dagegen ist der Unterarm eines bei Bukoba von Emin gesammelten 우 noch kürzer als derjenige des Togo-or; dieses Stück ist trocken praeparirt worden, die Gaumenfalten waren zerstört. Die Flughaut setzt sich an die Mitte des Rückens der ersten Phalanx der zweiten Zehe an, wie es für Ep. franqueti beschrieben wird und wie es auch bei den Togo-Exemplaren der Fall ist.

$E$. franqueti ist offenbar sehr nahe mit $E$. comptus verwandt und darf vielleicht gar nicht artlich von ihm geschieden werden; da sie neben einander am Gabun, im nordöstlichen Congo-Gebiet und an der Goldküste leben, so ist es nicht ausgeschlossen, dass beide zu einer und derselben Art gehören.

1. Ep. franqueti Tomes. - 1860. Epomophorus franqueti Tomes vom Gabun, P.Z. S. 1860, p. 54 -55, Taf. LXXV. (Thier). - 1861. Tomes, P. Z. S. Taf. I. Fig. 3, 3a, 3b (Schädel). -- 1878. Dobson, 1. c. p. 12-13, Taf. II., Fig. 4 (Gaumen). - 1880. Dobson, Rep. Chir. p. 4. - 1880. A. Smith, Proc. Roy. Phys. Soc. Edinb. p. 362-369. - 1881. Jentink, Not. Leyd. Mus. III. p. 62.

- 1881. Dobson, P. Z. S. p. 686-692 (Anatomie). - 1887. Jentink, Not. 
Leyd. Mus. X. p. 50. - 1888. Jentink, Cat. Syst. p. 137. - 1888. Thomas P. Z. S. p. 7 und 16. - 1894. Matschie, Mitth. Geogr. Ges. Lübeck p. 133. - 1896. de Pousargues, Ann. Sc. Nat. III. p. 254-255. - 1897. Trouessart 1. c. p. 89.

Hab. Sierra Leone (Dobson), Liberia, Goldküste (Jentink), Togoland (Berliner Museum), Gabun (Dobson), Tingasi in Monbuttu (Thomas), Bukoba (Matschie).

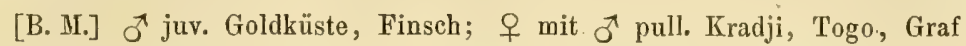
Zech; ơ juv. Fanti, Sharpe; 우 Bukoba, Emin Pascha. - [Stuttgarter Museum] ơ ad. Cape coast; $\widehat{\jmath}$ juv. Christiansborg an der Goldküste.

2. Ep. comptus Allen. - 1861. Ep. comptus Allen aus Duchaillu's Sammlungen, also vom Gabun, Pr. Ac. Nat. Sc. Phil. p. 158-159. - 1876. Ep. comptus, gambianus und franqueti, Peters, Monatsb. Akad. Berl. p. 474. - 1878. Dobson l. c. p. 13, Taf. II, Fig. 5 (Gaumen). - 1878. Dobson, P. Z. S. p. 879. 1887. Jentink l. c. p. 252. - 1888. Jentink l. c. p. 137-138. - 1891. Matschie, Arch. Naturg. I. 3 p. 353. - 1894, Matschie, Mitth. Geogr. Ges. Lübeck p. 133. - 1895. Matschie, Säugeth. D. 0. A. p. 16. - 1896. de Pousargues, Ann. Sc. Nat. III. p. 254 -255. - 1897. Sjöstedt, Mitth. Deutsch. Schutzgeb. X. p. 7. 1897. Sjöstedt, Bih. K. Sv. Vet. Acad. Handl. Bd. 23. Afd. IV. Nr. 1 p. 13, 15, 46. - 1897. Trouessart 1. c. p. 89. - 1897. Matschie, Arch. Naturg. I. 1 p. 83 .

Hab. Goldküiste (Jentink), Lagos (Dobson), Alt-Calabar (Dobson); Kamerun [Victoria, Mungo, Aqua Town, Kribi, Yaunde, Itoki (B. M. und Sjöstedt)], San Benito (de Pousargues), Dongila und Elloby am Gabun (B. M. und Dobson), Ogowe (Dobson), Malandje in Angola (B. Mr.), Semmio in Niam-Niam (Jentink).

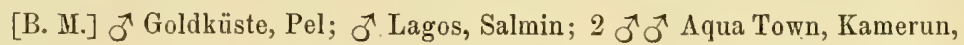
Reichenow; $\widetilde{T}$ Victoria, Kamerun, Preuss; $\widetilde{T}$ Mungo, Kamerun, Buchbolz;

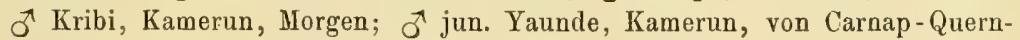
heimb; 우, 우 pull. Dongila, Gabun, Buchholz; đ̋ Malandje, Angola, von Mechow.

3. Ep. dobsonii Bocage. - 1889. Ep. dobsonii Barboza du Bocage von Quindumbo, Jorn. Sc. Math. Phys. Nat. Lisboa, 2. ser. I. p. 1--2, Fig. 1 (Gaumen), p. 14-15. - 1896. von Hanha, Bocage 1. c. p. 106. - 1897. Bocage 1. c. p. 188. - 1897. Trouessart 1. c. p. 88. - 1898. Bocage 1. c. p. 136, Fig. 2 (Gaumen).

Hab. Quindumbo, Galanga, Hanha in Angola.

4. Subgen. Micropteropus Mtsch. subgen. nov.

Die Entfernung zwischen dem vorderen Augenwinkel und der Nasenspitze ist ungefähr so gross, wie die Breite des Gesichtes an den Mundwinkeln. Der Gaumen trägt keine Querfalten, sondern eine nach vorn gegabelte Längsfurche in der Mitte, deren Ränder in dem nicht gegabelten Theile von 4 sehr dicht aneinander stehenden, mehr oder weniger deutlichen Höckern besetzt sind. Die Lippe ist nicht gefurcht. Der Schwanz ist kaum zu fühlen. Auf dem Plagiopatagium durchschneiden weniger als 32 Fascien den vom Ellenbogen zur Spitze des fünften Fingers vẹrlaufenden Strang. Die Reihe der Molaren ist viel länger 
als die Entfernung zwischen den Spitzen der oberen Caninen; der letzte obere Molar reicht weit ïber das Foramen infraorbitale nach hinten. Der knöcherne Gaumen ist ungefähr wie bei Epomops gebildet. Der Unterarm ist höchstens $60 \mathrm{~mm}$ lang.

Typus: Ep. pusillus Ptrs.

Hab. West-Afrika: Ober- und Nieder-Guinea, Congo-Gebiet, Seeen-Gebiet, Bongo im Gebiet des Weissen Niel.

Einzige Art: Ep. pusillus Ptrs. - 1860. Ep. schoensis Tomes (nec Rüpp.) vom Gambia und Gabun, P. Z. S. p. 56-58. - 1861. 'Tomes P. Z. S. p. 12, Taf. I. Fig. 4 und 4 a (Schädel). - 1867. Ep. pusillus Peters von Yoruba, Monatsb. Akad. Berlin p. 870. - 1870. Bocage, Jorn. Ac. Sc. Lisboa, III. Nr. X. p. 123. - 1878. Dobson 1. c. p. 14 -15, Taf. II. Fig. 6 (Gaumen). - 1881. Peters, Sitzb. Ges. naturf. Fr. Berlin p. 132. - 1887. Jentink 1. c. p. 252. - 1888. Jentink 1. c. p.138. - 1888. Jentink, Not. Leyd. Mus. X. p. 51. - 1889. Bocage, Jorn. Ac. Sc. Lisboa 2. ser. I. p. 15. - 1889. Noack, Zool. Jahrb. IV. p. 206-209, Taf. V. Fig. 54 (Schädel) und 55 (Gaumen). - 1890. Thomas, P. Z. S. p. 446. - 1893. Matschie, Mitth. Schutzgeb. VI. p.6. - 1894. Matschie, Mitth. Geogr. Ges. Lübeck p. 133. - 1896. de Pousargues, Ann. Sc. Nat. III. p. 255 - 256. - 1897. Trouessart 1. c. p. 89. - 1897. Sjöstedt, Bih. K. Sv. Vet.-Akad. Handl. Bd. 23, Afd. IV. Nr. 1 p. 46. - 1897. Sjöstedt, Mitth. Deutsch. Schutzgeb. X. p. 9. - 1898. Bocage l. c. p. 137.

Hab. Gambia (Tomes); Liberia [Grand Cape Mount und Little Cola] (Jentink); Togo [Kradji, Misahöhe, Klein-P opo und Bis marckburg] (Berliner Museum), Abbeokutu in Yoruba, Lagos (Dobson); Yaunde in Kamerun (Zenker); Congo Français (de Pousargues); Pungo Andongo, Tschintschoscho, Gabun, Malandje (Berliner Museum); Banana, Netonna (Noack); Nord-Angola (Bocage); Kemo-Fluss (de Pousargues); Kiriamo in der Nähe des Albert-Edward-See's (Thomas): Ngoroine zwischen Victoria-Nyansa und dem Guasso-Nyiro des Massailandes (0. Neumann); Bongo [Stuttgarter Museum, von Heuglin als Ep. anurus jung].

[B. MI.] $\sigma^{\nwarrow}$ juv., 2 우오 Bismarckburg, Conradt und Büttner; $\sigma^{\nwarrow}$ Kradji, Graf Zeeh; $\sigma^{\lambda}$ Misahöhe, Baumann; $\delta^{7}$ ㅇ Lagos, durch Gerrard; 우 Yoruba, Krauss; $\sigma^{\nearrow}$ Klein-Popo, durch die Linnaea; 2 ð Yaunde, Zenker; $\sigma^{\nearrow}$ Malandje, von Mechow; $\sigma^{\top}$ Tschintschoscho, Falkenstein; $\sigma^{\nearrow}$ Pungo Andongo, Falkenstein; 2 우 Gabun, Umlauff; 2 우 jun. Ngoroine, 0 . Neumann.

5. Subgen. Nanonycteris Mtsch. subgen. nov.

Die Entfernung zwischen dem vorderen Augenwinkel und der Nasenspitze ist viel grösser als die Breite des Gesichtes an den Mundwinkeln. Anf dem Gaumen stehen parallele Querfalten. Die Lippe ist nicht gefurcht. Der Schwanz ist noch kürzer als bei Micropteropus. Auf dem Plagiopatagium durchschneiden weniger als 32 Fascien dem vom Ellenbogen zur Spitze des fünften Fingers verlaufenden Strang. Die Reihe der Molaren ist nur so lang wie die Entfernung zwischen den Spitzen der oberen Caninen; der letzte obere Molar reicht nur bis zur Höhe des Vorderrandes des Foramen infraorbitale. Der knöcherne Gaumen ist wie bei Epomops gebildet. Der Unterarm ist kürzer als $60 \mathrm{~mm}$.

Typus: Ep. veldkampi Jent.

Hab. Ober- und Mittel-Guinea. 
Einzige Art: Ep. veldkampii Jent. - 1888. Ep. veldkampü Jentink von Buluma, Fisherman Lake in Liberia, Not. Leyd. Mus. X. p. 51-52. - 1894. Matschie, Mitth. Geogr. Ges. Lübeck p. 133. - 1897. 'Trouessart 1. c. p. 89.

Hab. Buluma in Liberia (Jentink); Accra; Bismarckburg, Misahöhe und und Kradji im Togoland; Lagos.

[B. M.] $\sigma^{\nearrow}$ Accra, Unger; 오 Bismarckburg, Conradt; 2 우 우 Misahöhe, Baumann; $\sigma^{\nearrow}$ juv. Kradji, Baumann; $\sigma^{\nearrow}$ Lagos durch Salmin.

\section{Genus. Xantharpyia Gray.}

1843. Xantharpyia J. E. Gray, List. Spec. Mamm. Brit. Mus. p. 37-38.

1844. Eleutherura Gray, Voy. Sulphur, Mammalia, p. 29. - 1852. Cynonycter is Peters, Reise Mossamb. Säugethiere p. 25. - 1860. Pterocyon Peters, Monatsb. Akad. Berlin p. 423. - 1869. Xantharpyia Fitzinger, Sitzb. Akad. Wien LX. 1. Abth. p. 71-90, October. - 1870. Senonycteris, Xantharpyia, Eleutherura Gray, Cat. Monk. Lem. Fruit-eating Bats. p. 115-119. - 1878. Cynonycteris Dobson, Cat. Chir. p. 70 -80, Taf. V. - 1880. Dobson, Rep. Brit. Assoc. p. 5-6. - 1887. Jentink in Mus. d'Hist. Nat. Pays-Bas IX. p. 263-264. - 1887. Xantharpyia, Blanford, P. Z. S. p. 636-637. - 1888. Cynonycteris Jentink, I. c. XII. p. 150-152. 1891. Barboza du Bocage, Jorn. Ac. Sc. Lisboa, 2. ser., II. p. 173-178. Fig. 1-3. - 1897. Trouessart, Cat. Mamm. Nov. Ed. I. p. 84-85. - 1898. Barboza du Bocage, Jorn. Ac. Sc. Lisboa, 2. ser. V. p. 133-135, 137-139.

Mittelgrosse Fledermäuse. Der Schwanz tritt deutlich aus der Behaarung hervor und ist mit der Schenkelflughaut verwachsen; das Endglied des Daumens ist an seiner Basis von der Flughaut umfasst, wie bei Epomophorus; der Zeigefinger trägt eine Kralle. Der Rand des Ohres bildet einen ovalen Ring, da sich der Aussenrand mit dem Innenrand an der Ansatzstelle des Ohres an den Kopf berührt. Die Flughaut setzt sich an den Rücken der zweiten Zehe an. Schnauze hundeartig gestreckt. Der Metacarpus des Mittelfingers ist ungefähr solang wie der Zeigefinger. Vor und hinter der Ohrwurzel kein Büschel weisser Haare. Gebiss: $\frac{2,1,3,2}{2,1,3,3}$. Oberlippe mit einer tiefen Grube zwischen den wulstig umrandeten Nasenlöchern und dem Lippenrande; die Ränder dieser Grube sind nicht wulstig erhöht.

Die oberen Schneidezähne stehen bogenförmig und sind von den Eckzähnen durch eine Lücke getrennt. Die Backenzähne sind bei jungen Thieren stumpfhöckerig mit einer Längsfurche auf der Krone und kauen sich mit dem Alter flach ab. Zwischen dem Eckzahn und den Praemolaren ist keine grössere Lücke. Der knöcherne Gaumen reicht weit über die Molarenreihe hinaus. - Die Saugwarzen befinden sich an den Seiten der Brust etwas unterhalb der Achselgrube. Die Ruthe des Männchens ohne Penisknochen. An den 
Halsseiten der Männchen (vielleicht nur zur Fortpflanzungszeit) ist unterhalb des Ohres ein kreisrundes Büschel borstiger Haare entwickelt, welche mit Drüsen in Verbindung stehen.

Die Weibchen sind im allgemeinen kleiner als die Männchen und haben einen kürzeren Kopf als diese. Die zur Gattung Xantharpyia gehörigen Thiere scheinen, nachdem sie das vollständige Gebiss erlangt haben, noch beträchtlich zu wachsen. Die Länge des Unterarms variirt. in weiteren Grenzen als bei Epomophorus und Xantharpyia verhält sich in dieser Hinsicht ähnlich wie Pteropus.

Die Bildung der Gaumenfalten ist bei allen mir bekannten Exemplaren ziemlich gleichförmig; stets kann man 7 Querfalten unterscheiden, von denen entweder die drei oder die vier letzten getheilt sind. Am Hinterrande des Gaumens stehen ausserdem entweder vier oder zwei oder nur eine gezähnelte Linie.

Das Plagiopatagium (die Ellenbogen-Flughant) zeigt eine äbnliche Vertheilung der Fascien wie bei der Gattung Epomophorus s. str.

Die Färbung der Xantharpyia-Arten ist bald graubraun, bald braungrau, bald strohgelb, je nach der Species.

Bei den Männchen hebt sich die Behaarung des Halses und der oberen Brust halsbandförmig ab, weil die Haare dort eine andere Richtung haben als auf dem Rücken und Bauch. Die Nachtflederhunde, wie man diese Arten nennen könnte, scheinen nur des Nachts zu fliegen. Sie leben sonst wie die Epomophorus und Pteropus, fressen Insekten und saftige Früchte und durchfliegen weite Strecken, um zu ihren Nahrungsbäumen zu gelangen. Nach Dobson sollen sie auch Weichthiere vom Meeresstrand aufnehmen.

Ihre Ruheplätze befinden sich im Gegensatz zu Pteropus und Epomophorus häufig in Felsspalten und Höhlen, gelegentlich aber auch auf Bäumen. Dobson ist der Ansicht, dass diejenigen Exemplare, welche Bäume bewohnen, sich durch lange Behaarung vor den Höhlenbewohnern auszeichnen. Ich vermuthe, dass die Behaarung je nach der Jahreszeit wechselt; weil ich aus den Sigi-Höhlen bei Tanga in Ostafrika sowohl langhaarige als kurzhaarige Individuen kennè.

Büttikofer erzählt (Not. Leyd. Mus. X. p. 52) von X. straminea, dass sie bis 12 Uhr Abends in ganzen Schwärmén um einen einzelstehenden Bombax flogen, von dem sie Blüthen und junge Triebe abfrassen, dass sie kein Geschrei hören liessen, angeschossen beim Fallen rasch aufeinander folgende piepende Rufe ausstiessen, dass sie sehr ruhig fliegen und sehr zählebig sind.

Es sind bis jetzt 25 Arten von Xantharpyia beschrieben worden; von diesen hat Trouessart 189710 als Arten angenommen. Ich erkenne 9 Arten an.

Gray hat 1870 die Arten dieser Gattung in zwei Gattungen Xantharpyia und Eleutherura vertheilt und von Xantharpyia als Untergattung noch Senonycteris abgesondert. Ich kann diese Eintheilung ebenso wenig annehmen, wie ich Dobson in der Zasammenziehung aller Arten zu einer Gruppe folgen möchte.

Ich schlage folgende Eintheilung vor:

Die Untergattung Pterocyon Ptrs. ist ausgezeichnet dadurch, dass der erste untere Molar so gross ist wie der zweite und der letzte untere Molar zusammengenommen, und dass auf der Ellenbogen-Flughaut, dem Plagiopatagium, mindestens 18 Fascien den vom Ellenbogen zur Spitze des fünften Fingers verlaufenden Strang schneiden. Auf dem Gaumen stehen binter den 7 Querfalten noch 4 gezähnelte Querlinien.

Bei der Untergattung Xantharpyia Gray ist der erste untere Molar kleiner als der zweite und der letzte Molar zusammengenommen, auf der Ellenbogen-Flughaut durchschneiden höchstens 16 Fascien den vom Ellenbogen zur Spitze des fünften Fingers verlaufenden Strang. Auf dem Gaumen ist nur eine spitzwinklig nach vorn gerichtete, gezähnelte Linie hinter den 7 Querfalten vorhanden. 
Beide Untergattungen stimmen darin überein, dass auf dem Gaumen hinter vier ungetheilten Querfalten drei getheilte Querfalten stehen und dass der Hinterfuss mit den Krallen länger als das $\mathrm{Ohr}$ ist.

$\mathrm{Zu}$ Pterocyon rechne ich $X$. straminea und dupreana, zu Xantharpyia aber X. collaris, aegyptiaca, amplexicaudata, brachycephala und brachyotis.

Es bleiben noch übrig $X$. torquata und angolensis. Bei diesen stehen auf dem Gaumen hinter drei ungetheilten Querfalten viẹr getheilte Querfalten, während zwei gezähnelte Linien den Abschluss des Gaumens nach hinten bilden, und der Fuss mit Kralle ist höchstens so lang wie das $\mathrm{Ohr}$.

Ich halte es für praktisch, diejenigen Arten von Xantharpyia, welche die eben erwähnten Merkmale besitzen, in einer besonderen Untergattung abzusondern, für welche ich den Namen Myonycteris von uvs und víxtepts vorschlage. Als Typus derselben betrachte ich Cynonycteris torquata Dobs.

Hab.: Madagaskar, Comoren, Afrika, südlich von der Sahara, Nubien, Aegypten, Cypern, Palaestina, Arabien, Süd-Asien, naeh Norden bis zum Himalaya, Malayischer Archipel, Neu-Guinea, Bismarck-Archipel, Salomons-Inseln.

\section{Uebersicht der Untergattungen.}

Das Ohr ist kürzer als der Hinterfuss mit den Krallen (letztere im Bogen gemessen); auf dem Gaumen befinden sich vier ungetheilte und drei getheilte Querfalten, hinter welchen entweder vier oder nur eine gezähnelte Linie sich befinden:

Mindestens 18 Fascien durchschneiden den vom Ellenbogen zur Spitze des fünften Fingers verlaufenden Strang; Kopf länger als $53 \mathrm{~mm}$; der erste untere Molar so lang wie der zweite und letzte untere Molar zusammengenommen . . . . . . . Pterocyon Ptrs. p. 62

Höchstens 16 Fascien durchschneiden den vom Ellenbogen zur Spitze des fünften Fingers verlaufenden Strang; Kopf kürzer als $50 \mathrm{~mm}$; der erste untere Molar ist kürzer als der zweite und letzte Molar

Das $\mathrm{Ohr}$ ist länger als der Hinterfuss mit den Krallen (letztere Xantharpyia Gray p. 65 im Bogen gemessen); auf dem Gaumen befinden sich drei ungetheilte und vier getheilte Querfalten; der erste untere Molar ist kürzer als der zweite und letzte Molar zusammengenommen; hinter den sieben Querfalten sind zwei gezähnelte Linien vorhanden. . . . . . . . . . Myonycteris Mtsch. p. 63 


\section{Uebersicht der geographischen Verbreitung.}

\begin{tabular}{|c|c|c|c|}
\hline & Pterocyon & Myonycteris & Xantharpyia \\
\hline $\begin{array}{l}\text { San Thomé } \\
\text { Guinea und Congo }\end{array}$ & $\begin{array}{l}\text { stramineus } \\
\text { stramineus }\end{array}$ & $\begin{array}{c}\text { torquata } \\
\text { und angolensis }\end{array}$ & $\begin{array}{c}\text { brachycephala } \\
\text { collaris }\end{array}$ \\
\hline Das übrige tropische Afrika & stramineus & - & collaris \\
\hline Madagassisches Gebiet & dupreanus & - & collaris \\
\hline $\begin{array}{c}\text { Nubien, Aegypten, Syrien, Cypern, } \\
\text { Palaestina }\end{array}$ & - & - & aegyptiaca \\
\hline Indisches Gebiet & - & - & amplexicaudata \\
\hline Celebes bis Salomons-Inseln & - & - & brachyotis \\
\hline
\end{tabular}

\section{Subgen. Pterocyon Ptrs.}

1860. Peters, M. B. Akad. Berlin, p. 423.

Der erste untere Molar ist so lang wie der zweite und der letzte untere Molar zusammengenommen. Der letzte obere Molar ist kaum doppelt so lang wie der erste obere Praemolar. Auf dem Plagiopatagium schneiden mindestens 18 Fascien den vom Ellenbogen zur Spitze des fünften Fingers verlaufenden Strang. Auf dem Gaumen stehen hinter 4 ungetheilten Querfalten drei getheilte Querfalten und hinter diesen mehr als 2 gezähnelte Querlinien. Das Ohr ist kürzer als der Hinterfuss mit den Krallen.

Typus: Pterocyon stramineus (Ptrs.)

Hab. Afrika, südlich von der Sahara, Madagaskar und Sïd-Arabien.

Uebersicht der Arten:

Behaarung der Rückenseiten gelblich . . . . . . . . . X. straminea p. 62 Behaarung der Rückenseiten graubraun .. . . . . . . . X. dupreana p. 63

1. X. straminea Geoffr. - 1810. Pteropus stramineus Geoffroy-Saint-Hilaire angeblich von Timor und Ternate. - 1827. Temminck, Monogr. Mammal. I. p. 195-197, Taf. XV. Fig. 12 (Gebiss von vorn), 13 (Schädel). - 1837. von Sennar, Temminck 1. c. II. p. 84-85. - 1843. Xantharpyia straminea Gray, List. Spec. Mamm. Brit. Mus. p. 38. - 1845. Hedenborg, Isis p. 581 . 1855. Cynonycteris straminea Wagner, Schreber's Säugethiere, Suppl. Bd. V. p. 603. - 1860. Pterocyon paleaceus Peters von Sennar, M. B. Akad. Berlin p. 423. - 1861. Pteropus mollipilosus H. Allen aus Duchaillu's Sammlungen, also vom Gabun, Pr: Ac. Nat. Sc. Philadelphia p. 159-160. - 1865. Pteropus palmarum Heuglin vom Weissen Nil, Verh. Leop. Carol. Akad. Heft V. Nr. 3 u. 4, p. 34. - 1866. Cynonycteris straminea M. B. Akad. Berlin p. 885. 1866. Xantharpyia leucomelas Fitzinger vom oberen Nil, Sitzb. Akad. Wien LIV. p. 8 Nr. 2 - 1868. Pterocyon paleaceus und leucomelas, Hartmann, Ztschr. 
Ges. Erdk. Berlin III. p. 40. - 1869. Xantharpyia palmarum, straminea und leucomelas Fitzinger l. c. p. 71-79. - 1876. Cynonycteris straminea Peters, M. B. Akad. Berlin p. 474. - 1878. Cynonycteris straminea Dobson 1. c. p. 77-78. - 1884. Greef, Sitzb. Ges. Naturw. Marburg p. 44. - 1886. Martinez y Saes, Anal. Soc. Esp. Hist. Nat. XV. p. 339. - 1887. Monticelli, An. Mus. Civ. St. Nat. Genova 2. ser. V. p. 524. - 1887. Jentink, Cat. Ost. p. 264. 1888. Jentink. Cat. Syst. p. 152. - 1888. Jentink, Not. Leyd. Müs. X. p. 52. 1889. Barboza du Bocage, Jorn. Sc. Math. Lisboa, 2. ser. I. p. 15. - 1891. Bocage, l. c. II. p. 173-175, Fig. 1 (Gaumen). - 1893. Matschie, Sitzb. Ges. naturf. Fr. p. 24. - 1895. Thomas, P. Z. S. p. 545. - 1895. Bocage 1. c. XIII. p. 4. - 1895. Matschie, Säugethiere D. 0. A. p. 17. - 1896. Thomas, P. Z. S. p. 790. - 1896. de Pousargues, Ann. Sc. Nat. Zool. III. p. 256-257. 1897. Thomas, P. Z. S. p. 927. - 1897. Sjöstedt, Bih. K. Sv. Vet. Akad. Handl. Bd. 23, Afd. IV. Nr. 1, p. 46. - 1897. Matschie, Arch. Naturg. Bd. I. Hft. 1, p. 83. - 1897. Trouessart 1. c. p. 85. - 1898. Bocage l. c. XIX. p. 135, 137.

Hab. Afrika, südlich von der Sahara, nach Siuden bis zum Zambese, Süd-Arabien, im besonderen Senegal (Jentink, Dobson); Gambia, Sierra Leone (Dobson), Liberia (Jentink); Guinea (Temminck, Esqu. Zool. Guiné 1853 p. 54-55); Aschante (Jentink); Whyda (Dobson); Kamerun [Itoki (Sjöstedt); B uea (Preuss); Bipindi und Yaunde (Zenker); Kamerunfluss (Buchholz)]; Fernando Po (Bocage); Isla do Principe (B. M.); San Thomé (Greef, Bocage); Gabun (Dobson); Cap Lopez (B. MI.); Französisch Congo (de Pousargues); Tschintschoscho (B. MI.); Benguella (B. M.); Ajuda, Rio Cuilo, Caconda (Bocage 1898); Nyassa-Land [Zomba (Thomas 1896); Mt. Malosa (Thomas 1897)]; Zanzibar-Küste (B. M.); Zanzibar (Dobson); Semmio in Niam-Niam (B. MI.); Sennar (Dobson, Jentink); Bahr el Abiad (Jentink); Lahadsch bei Aden (Natschie, Thomas, Monticelli).

[B. M.] $\sigma^{\nearrow ~ K a m e r u n, ~ B u c h h o l z ; ~} \sigma^{\nearrow ~ I t o k i, ~ K a m e r u n, ~ S j o ̈ s t e d t ; ~} ᄋ$ Yaunde, Zenker; 오 Bipindi, Zenker; 2 우 Buea, Preuss; $\sigma^{\nearrow}$ Fernando Po, Frank;

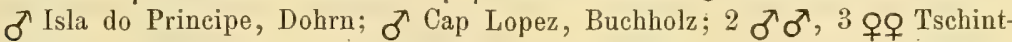
schoscho, Falkenstein; $3 \delta^{\top} \sigma^{\top}$ Semmio, Bohndorff; $\sigma^{\pi}$ Sennar, Heuglin; 오 Benguella, Monteiro; $ᄋ$ Zanzibar-Küste, von der Decken; 우 - Frank; ㅇ - Leydener Museum, wahrscheinlich eines der Temminck'schen OriginalExemplare; $3 \sigma^{\nearrow}$, 3 우 우 Zoologischer Garten und Aquarium.

2. X. dupreana Pollen. - 1866. Pteropus dupréanus von Nordwest-Madagaskar, Pollen bei Schlegel, P. Z. S. p. 418-419. - 1878. Dobson 1. c. p. 78. - 1879. Bartlett, P. L. S. p. 769. - 1887. Jentink l. c. p. 264. - 1888. Jentink l. c. p. 152 . - 1897. Trouessart 1. c. p. 85.

Hab. Madagaskar.

[B. M.] O Südost-Madagaskar, Bartlett.

2. Subgen. Myonycteris Mtsch. subgen. nov.

Dè erste untere Molar ist kürzer als der zweite und der letzte untere Molar zusammengenommen. Der letzte obere Molar ist ungefähr ${ }^{2 / 3}$ so lang wie der vorletzte obere Molar. Der erste obere Praemolar ist kaum grösser als ein oberer Incisivus. Der erste untere Praemolar ist sehr klein, nur etwas grösser als ein unterer Schneidezahn. Auf dem 
Gaumen stehen hinter. drei ungetheilten Querfalten vier getheilte Querfalten, und zwei gezähnelte Linien bilden den Abschluss des Gaumens nach hinten. Der Fuss mit den Krallen ist höchstens so lang wie das Ohr. Auf dem Plagiopatagium durchschneiden höchstens 16 Fascien den vom Ellenbogen zur Spitze des fünften Fingers verlaufenden Strang.

Typus: Cynonycteris torquata Dobson.

Hab. Pungo Andongo, Cahata, Quibula, sämmtlich in Angola (Bocage), Angola (Wellwich nach Gray), Bismarckburg in Togo (B. M.); Liberia (Jentink); Bukoba am Victoria-Nyansa (B. M.).

Uebersicht der Arten:

Unterarm länger als $70 \mathrm{~mm}$ :

$X$. angolensis

Unterarm kürzer als $60 \mathrm{~mm}$ :

X. torquata.

Ich habe torquata Dobs. bis jetzt noch nicht vergleichen können. und betrachte deshalb vorläufig noch beide Formen als besondere Arten, obwohl ich vermuthe; dass beide zu einer Art gehören. Die Exemplare von Xantharpyia variiren in den Grössenverhältnissen nicht unbedentend und die Bildung eines Halskragens mag vielleicht nur den Männchen in der Fortpflanzungszeit zukommen.

1. X. torquata Dobson. - 1878. Cynonycteris torquata Dobson von Angola (durch Wellwich gesammelt), Cat. Chiroptera p. 76-77, Tafel V. Fig. 1. (Thier). - 1888. Jentink, Not. Leyd. Mus. X. p. 52.

Hab, Angola (Dobson); Liberia [Schieffelinsville, Junk River (Jentink)].

2. X. angolensis Bocage. - 1898. Cynonycteris angolensis von Pungo Andongo im Norden des Cuanza, von Cahata und Quibula im Innern von Benguella, Barboza du Bocage, Jorn. Ac. Sc. Lisboa, 2. ser. V. p. 133-135, 138, Fig. 1 (Gaumen). - 1889. Cynonycteris aegyptiaca Barboza du Bocage, Jorn. Ac. Sc. Lisboa, 2. ser. I. p. 15. - 1892. Cynonycteris sp.? Bocage, Jorn. Ac. Sc. Lisboa, 2. ser. II. p. 174-176, Fig. 2 (Gaumen). - 1893. Cyn. torquata Matschie, Mitth. Deutsch. Schutzgeb. Heft 3, p. 7. - 1895. X. collaris partim Matschie, Säugeth. D. 0. A. p. 17.

In meinem Buche: Säugethiere Deutsch-Ost-Afrika's habe ich $X$. collaris für Bukoba erwähnt. Das eine der drei im Berliner Museum von diesem Fundorte aufbewahrten Exemplare gehört, wie mich eine genauere Untersuchung belehrt hat, zu Myonycteris. Die Färbung dieses Stückes ist auf den Kopf und Oberrücken graubraun, auf den Unterrücken dunkler und in der Sacralgegend rostfarbig überflogen. Der Vorderhals ist hellgran; die übrige Unterseite braungrau. Das Exemplar, ein of mit grossen Brustwarzen ist am 15. XII. 1890 bei Bukoba gesammelt worden; der Unterarm ist $74 \mathrm{~mm}$ lang. Die Behaarung ist dicht und mässig lang.

Das alte Weibchen von Togo, welches im Berliner Museum vorhanden ist, hat einen Unterarm von $72 \mathrm{~mm}$ Länge.

Hab. Pungo Andungo, Cahata, Quibula (Bocage); Bismarckburg in Togo (B. M.), Bukoba am Victoria-Nyansa (B. M.). Pascha.

[B. M.] 오, 2 ठð $\sigma^{\Uparrow}$ pull. Bismarckburg in Togo, Büttner; 우 Bukoba, Emin 


\section{Subgen. Xantharpyia Gray.}

1821. Rousettus Gray, London Medical Repository XV., p. 299, April 1 [fide T. S. Palmer, Proc. Biol. Soc. Washington, 1898, XII., p. 112]* — 1843. Xantharpyia Gray, List of the Specimens of Mammalia in the Collection of the British Museum. London p. 37. - 1898. Cynonycteris** F. de Seabra, Jorn. Lisboa p. 155-162, 169-170, Taf. I, Fig. 9-14 (Gaumen).

Der erste untere Molar ist kürzer als der zweite und letzte untere Molar zusammengenommen. Der letzte obere Molar ist ungefähr $2 / 3$ so lang wie der vorletzte obere Molar. Der erste obere Praemolar ist kaum grösser als ein oberer Incisivus. Der erste untere Praemolar ist doppelt so gross wie ein unterer Incisivus. Auf dem Gaumen stehen hinter vier ungetheilten Querfalten drei getheilte Querfalten und eine einzige, spitzwinklig nach vorn gerichtete, gezähnelte Linie schliesst den Gaumen nach hinten ab. Der Fuss mit den Krallen ist länger als das Ohr. Auf dem Plagiopatagium durchschneiden höchstens 16 Fascien den vom Ellenbogen zur Spitze des fünften Fingers verlaufenden Strang.

Typus: Xantharpyia amplexicaudata (Geoffr.)

Hab. Tropische und subtropische Gebiete der alten Welt. Noch nicht nachgewiesen in Nord-Afrika westlich von Aegypten, in Europa, in Asien nördlich von der Nordgrenze des Indischen Gebietes, in Australien, Micronesien und in Melanesien, südlich vom $10{ }^{\circ}$ südl. Breite.

Uebersicht der Arten.

Der letzte obere Molar ist ungefähr so gross wie der erste obere

Praemolar

X. brachycephala p. 66

Der letzte obere Molar ist viel grösser als der erste obere Praemolar:

Die Länge der Ohren ist gleich der Entfernung des vorderen Augenrandes von der Nasenspitze . . . .

Die Ohren sind viel länger als die Entfernung des vorderen Augenrandes von der Nasenspitze:

Auf dem Gaumen ist die vorletzte Querfalte gabel-

förmig getheilt: . . . . . . . . . .

Auf dem Gaumen ist die vorletzte Querfalte einfach:

Wenn der zweite Finger mit der Kralle nicht mehr über den Metacarpus des dritten Fingers hinaus reicht, wenn auf der Krone der Molaren eine Längsfurche nicht mehr sichtbar ist und die Epiphysen der Fingergelenke mit den Phalangen-Enden verwachsen sind, so ist der Unterarm mindestens $86 \mathrm{~mm}$ lang: . . . . . . X. collaris p. 66 ist der Unterarm kürzer als $86 \mathrm{~mm}$ : X.amplexicaudata ${ }^{* * *} \mathrm{p} .67$

* Da ich die Arbeit von Gray aus dem Jahre 1821 vorläufig noch nicht einzusehen im Stande bin, und andererseits der Bogen 4 meiner Arbeit schon abgesetzt worden ist, so behalte ich hier den Namen: Xantharpyia vorläufig bei.

** Cynonycteris grandidieri s. u. Cynopterus.

* Bei jungen Thieren Unterschiede zwischen den afrikanischen und asiatischen $\mathrm{X}$. anzugeben, ist mir nicht gelungen. Die Behaarung der Schenkel ist bei beiden dieselbe. 
1. X: brachycephala Bocage. - 1889. Cynonycteris brachycephala von San Thomé, Barboza du Bocage, Jorn. Sc. Math. Phys. Lisboa, 2. ser. I. p. 197-198. - 1897. Trouessart 1. c. p. 84. - 1898. Bocage l. c. V. p. 138 -139, Fig. 6 (Gaumen). - 1898. F. de Seabra, 1. c. p. 159, 170 Taf. I, Fig. 12 (Gaumen).

Hab. San Thomé.

2. X. collaris Illiger. - 1815. Pteropus collaris Illiger vom Vorgebirge der guten Hoffnung, Abh. Berlin. Akad. p. 84. - 1829. Pt. leachii von Capstadt, Smith, Zoolog. Journ. IV. p. 433. - 1837. Pt. hottentottus vom Cap der guten Hoffnung, Temminck, Monogr. Mamm. II. p. 87--88, Taf. XXXVI. Fig. 16. und 17 (Schädel). - 1844. Eleutherura hottentotta Gray, Voy. Sulphur, Mamm. p. 29. - 1852. Cynonycteris collaris. Peters, Reise Mossamb. Säugethiere p. 25. - 1869. Xantharpyia leachii und hottentotta Fitzinger 1. c. p. 82-86. - 1870. Eleutherura unicolor vom Gabun, Gray, Cat. Monk. Lem. Fruit-eating Bats. p. 117. - 1878. Dobson 1. c. p. $75-76$. - 1881. Anderson, Cat. Mamm. Ind. Mus. I. p. 104. - 1887. Jentink 1. c. p. 263. - 1888. Jentink 1. c. p. 151. - 1891. Cynon. unicolor Matschie, Arch. Naturg. I. 3, p. 351. 1891. C. spec. Bocage, Jorn. Sc. Math. Lisboa, 2. ser. II. p. 177, Fig. 3 (Gaumen). - 1895. C. collaris Sjöstedt, Kgl. Sv. Vet. Akad. Handl. Bd. 27, Nr. 1. p. 13 u. 18. - 1895. Matschie, Säugethiere D. 0. A. p. 17. - (?) 1895. X. aegyptiaca Thomas, P. Z. S. p. 545. - 1897. Sjöstedt, Mitth. Deutsch. Schutzgeb. X. p. 7. - 1897. Matschie, Arch. Naturg. I. 1, p. 83. - 1897. Sjöstedt, Bih. Kgl. Sv. Vet. Akad, Handl. Bd. 23, Afd. IV. Nr. 1, p. 13, 15, 16, 46. - 1897. Trouessart 1. c. p. 84. - 1898. Barboza du Bocage 1. c. XIX. p. 137, Fig. 4 (Gaumen). 1898. F. de Seabra, I. c. p. 159, 169, Taf. I, Fig. 14 (Gaumen).

Die mir bekannten ostafrikanischen Exemplare sind sehr dunkel und zum Theil sehr lang und dicht behaart. Ein sehr dicht behaartes Exemplar ist am 18. VI. am Runssoro erlegt, die übrigen stammen von Tanga, haben aber keine Datumsangaben. $\mathrm{Ob}$ die südafrikanischen $X$. collaris immer sehr helle Flughäute haben, und $o b$ die westafrikanischen immer grösser sind als die südafrikanischen Stücke, vermag ich nach unserem Material noch nicht zu entscheiden.

Hab. Afrika, siidlich von der Sahara, und Anjuan auf den Comoren: im besonderen Capland, Natal (Dobson), Inhambane (B. MI.); Mossambik (Bocage 1898); Tanga in Deutsch-Ost-Afrika (B. M.); Bukoba (B. M.); Karevia in Ukondjo am Runssoro (B. MI.); Buea auf dem Kamerun-Gebirge (B. M.); Gabun (Dobson); Pungo Andongo und Quindumbo in Angola (Bocage 1898), vielleicht auch Aden (Thomas 1895).

[B. M.] $\sigma^{\pi}, 2$ 우 Capland, Mund, Krebs und Leydener Museum (Pt. hotten-

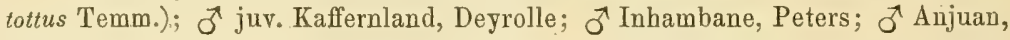
Hildebrandt; $\delta^{\top}$ ㅇ Pangani, Gerrard; $3 \delta^{\star} \sigma^{\top}, 4$ 우 우 Tanga, 0. Neumann und Martienssen; $\delta^{\nearrow}$, ㅇ Bukoba am Victoria-Nyansa, Emin Pascha; . Karevia in Ukondjo am Runssoro, Emin Pascha; $\delta$ juv., $\&$ Buea auf dem KamerunGebirge, Preuss.

3. X. aegyptiaca Geoffr. - 1810. Pteropus aegyptiacus von Gizeh bei Cairo, Geoffroy St. Hilaire, Descr. de l'Egypte. Hist. Nat. II. Mamm. p. 134-136, Taf. III. Fig. 2 (Thier). - 1827. Pteropus geoffroyi von Aegypten und vom 
Senegal, Temminck, Monogr. Mamm. I. p. 197-198, Taf. 15, Fig. 14-15 (Gebiss von vorn und Schädel). - 1869. Fitzinger l. c. p. 79-81. - 1878. Dobson l. c. p. 74-75. - 1879. C. collaris Günther, P. Z. S. p. 741. - 1880. Dobson, Rep. Brit. Ass. 1880 p. 5. - 1887. C. aegyptiaca Jentink, 1. c. p. 263. - 1888. Jentink 1. c. p. 151. - 1891. Noack, Jahrb. Hamb. Wiss. Anst. IX. p. 56-57. - 1897. Trouessart l. c. p. 84. - 1898. F. de Seabra, Jorn. Lisboa p. 158, 169.

Hab. Nubien (Hartmann, Zeitschr. Ges. Erdk. Berlin 1868 p. 40), Aegypten, Abessynien (!), Senegal (!), Palaestina, Cypern (Dobson), Syrien (Sealıra).

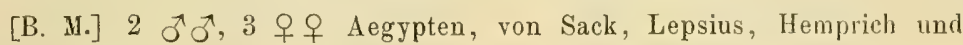
Ehrenberg; $\sigma^{\Uparrow}$ Palaestina, Schlüter; $3 \precsim \widehat{\jmath}, 1$ 우 Cypern, Rolle.

4. X. amplexicaudata Geoffroy. - 1810. Pteropus amplexicaudatus von Timor, Geoffroy-Saint-Hilaire, Ann. Mus. d'Hist. Nat. XV. p. 96-97, Taf. IV (Thier). - 1821. Pteropus leschenaultii von Pondicherry, Desmarest, Mammal. p. 110. - 1835. Pt. pyrivorus von Nepal, Hodgson, Journ. Asiat. Soc. Bengalen p. 700. - 1837. Pt. amplexicaudatus Temminck, Monogr. Mamm. II. p. 90, Taf. 36, Nr. 18--19 (Schädel). - 1843. Xantharpyia amplexicaudata und Cynopterus affinis vom Himalaya, Gray, List. Spec. Mamm. Brit. Mus. p. 39. - 1852. Pt. seminudus von Ceylon, Kelaart, Journ. Asiat. Soc. Beng. p. 345. - 1855. Cynonycteris amplexicaudata Wagner, Schreber's Säugethiere, Suppl. Bd. V. p. 604. - 1869. Xantharpyia amplexicaudata und leschenaultii Fitzinger 1. c. p. 86-90. - 1870. Senonycteris seminuda; Eleutherura marginata von Nepal; E. futiginosa von. Siam, Lao Mountains; E. infumata von Flores (?); E. fusca von Indien (?); E. philippensis von Manilla, Gray, Cat. Monk. Lem. Fruit-eating Bats, p. 115, 118-119. - 1873. Cynonycteris infuscata von Calcutta, Peters, M. B. Akad. Berlin 1873 p. 487. - 1873 (?) Cynonycteris minor von Java, Dobson, Journ. Asiat. Soc. Beng. p. 203, Taf. XIV. Fig. 9 (Ohr). 1878. C. amplexicaudata und (?) minor Dobson, Cat. Chiropt. p. 72-74. 1880. C. amplexicaudata Dobson, Rep. Biit. Ass. 1880 p. 5. - 1881. C. amplexicaudata und (?) minor Anderson, Cat. Mamm. Ind. Mus. I. p. 103-104. 1887. C. amplexicaudata Jentink 1. c. p. 263. - 1888. Jentink l. c. p. 150-151. 1890. Jentink, Weber's Zoolog. Ergebn. Niederl. Ost-Indien I. p. 126. - 1891. Xantharpyia amplexicaudata Blanford, Fauna Brit. Ind. Mamm. p. 261-262, Fig. 76 (Ohr). - 1892. Thomas, An. Mus. Civ. St. Nat. Genova, 2. ser. X. p. 921. - 1893. Everett, P. Z. S. p. 494. - 1894. Thomas, P. Z. S. p. 449. 1895. Thomas, An. Mus. Civ. St. Nat. Genova, 2. ser. XIV. p. 108. - 1896. Elliot, Field Columb. Mus. Zool. Ser. vol. I. Nr. 3, p. 79. - 1897. Thomas, Nov. Zool. IV. p. 263. - 1897. Trouessart 1. c. p. 84. - 1898. F. de Seabra, Jorn. Lisboa, 2. ser. V, p. 160, 161, 168, Taf. I, Fig. 10 (Gaumen). - (?) Cynonycteris bocagei von D yli auf Timor, F. de Seabra, I. c. p. 157-158, 160-161, 169, Taf. I, Fig. 11 (Gaumen). - 1898. Hartert, Nov. Zool. V, p. 456.

Hab. Von den Uferländern des Persischen Golfes und Arabischen Meeres nach Osten bis zu den Philippinen, Borneo, Java, Flores und Timor, nach Norden bis zum Himalaya und den Quellländern der südlichen Nebenflüsse des Jang tse kiang; im besonderen: Maskat (Thomas 1894), Kishm-Insel im Persischen Golf (Blanford, Eastern Persia 1876 p. 18-19), Vorder- und Hinter- 
indien (Dobson), Amoy in China (Jentink 1888), Engano (Thomas 1892), Sumatra (Dobson), Java (Jentink 1888), Timor (Jentink 1888, Seabra 1898), Flores (Heude, Mém. Hist. Nat. Emp. Chin. 1896 III, p. 179 Anmerkung), Luzon (B. M.), Negros (Elliot), (?) Aru-Inseln (B. M.).

[B. M.] $\sigma^{\nearrow}$ juv., 3 우우 Ceylon, Cuming und Nietner; $\delta^{\top}$ 우 Madras, Mitchell; ㅇ Bengalen, Lamare Piquot; $q$ Calcutta, 一; ðౌ Burma, Day; $\delta^{\nwarrow}$ Hongkong, Schoenlein; ㅇ Bangkok, von Martens; đ Samar, Jagor; 5 우 Jamtik, S. 0. Luzon, Jagor; ð Aru-Inseln (?), Gerrard:.

5. X. brachyotis Dobson. - 1877. Cynonycteris brachyotis von Neu-Mecklenburg, Dobson, P.Z.S. p. 116-117. - 1878. Dobson 1. c. p. 74, Taf. V. Fig. 2. (Kopf). - 1883. Jentink, Not. Leyd. Mus. V. p. 173. - 1887. Thomas, P. Z. S. p. 323. - 1888. Jentink l. c. p. 151. - 1888. Thomas, P. Z. S. p. 483-484. - 1897. Trouessart 1. c. p. 84.

Hab. Celebes (Jentink), Neu - Mecklenburg (Dobson), Salomons - Inseln (Thomas 1887). Neu-Lauenburg (B. M.), Neu-Guinea (B. MI.).

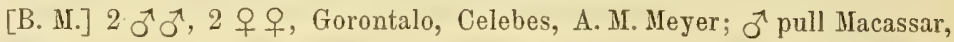
Celebes, Conrad; + N Neu-Lauenburg, Gerrard; ð̋ Arfak, Neu-Guinea, Bruijn.

* Wenn die Fundortsangabe für dieses Stück richtig ist, so würde X. amplexicaudata in das Gebiet von $X$. brachyotis erbeblich übergreifen. Jentink (1888) giebt allerdings schon ein o juv. in Alcohol von $X$. amplexicaudata für Celebes an, welches ebenfalls im Wohngebiete von X. brachyotis liegt. Es wäre sehr interessant, die Unterschiede zwischen den Celebes-brachyotis und amplexicaudata genau festzustellen. Das Fehlen des ersten Praemolars bildet keinen Speciesunterschied und kommt gelegentlich auch bei Exemplaren in Vorderindien vor. Von Wichtigkeit dürften aber die geringe Länge des Ohres und die sehr dunklen Flughäute sein. Es scheint auch, als ob brachyotis wesentlich kleiner als amplexicaudata wäre. $\mathrm{Ob} X$. minor, welche ich mit einem Fragezeichen zu $X$. amplexicaudata stellte, wirklich nur das Junge zu dieser Art ist, muss eine genaue Untersuchung des Original-Exemplares entscheiden. Dass der erste Molar. zwischen Eckzahn und zweiten Praemolar eingezwängt ist, kommt auch bei Philippinen - und binterindischen Exemplaren vor. Ob $X$. amplexicaudata in Vorderindien etwas anders aussieht als in Hinterindien und ob die hinterindische Form sich von der südchinesischen unterscheidet, kann ich noch nicht sagen. Das Berliner Museum hat ein sehr dunkles Exemplar mit dunkeln Flügeln aus China; auch die Stücke von den Philippinen zeichnen sich durch gewisse Merkmale (kleiner Zwischenraum zwischen dem Caninus und zweiten Praemolar, den der erste Praemolar fast ganz ausfüllt, oblonge Form des ersten unteren Praemolar, welcher sehr nahe an den zweiten Praemolar herantritt) vor den vorderindischen aus. Ein einziges Stück von den Philippinen zeigt aber diese Merkmale nicht und deshalb bleibt die Möglichkeit offen, dass $X$. amplexicaudata im allgemeinen sehr variirt. C. bocagei E. de Seabra von Timor habe ich nicht gesehen; die Länge der Ohren $(36 \mathrm{~mm})$ weist darauf hin, dass diese Species mit brachyotis nichts zu thun hat; sie soll sich von amplexicaudata durch folgende Merkmale unterscheiden: der Schädel soll breiter und kürzer, der Oberkiefer länger und der Gaumen nach hinten stark verschmälert sein. Herr F. de Seabra hat nicht angegeben, ob der von ihm abgebildete Schädel von amplexicaudata einem ơ oder 우 angehört hat. Ich vermuthe, dass er einem $\widehat{\sigma}$ angehört, und glaube, dass man C. bocagei so lange für identisch mit $C$. amplexicaudata halten darf, bis die Unterschiede dieser neuen Species von dem $0^{t}$ der $C$. amplexicaudata aus Timor nachgewiesen worden sind. 


\title{
5. Genus. Boneia Jentink.
}

\author{
1879. Boneia Jentink, Not. Leyd. Mus. I, p. 117-119.
}

Grosse Fledermäuse mit wohl entwickeltem, ziemlich dicken, z. Th. in die Flughaut eingeschlossenem Schwanz; der Zeigefinger trägt eine Kralle. Der Rand des Ohres bilden einen ovalen Ring, da sich der Aussenrand mit dem Innenrand an der Ansatzstelle des 0hres an den Kopf berührt. Die Flughaut setzt sich in der Mitte zwischen den Metatarsalien der ersten und zweiten Zehe an. Die Schnauze ist hundeartig. Der Metacarpus des Mittelfingers ist wenig kürzer als der Zeigefinger mit der Kralle. Oberlippe mit einer tiefen Grube zwischen den wulstig umrandeten Nasenlöchern; Unterlippe gleichfalls tief gefurcht. Äusserer Ohrrand an der Basis mit einem kleinen vorspringenden, verdickten Lappen. Die Augen sind von den Nasenlöchern und Ohren gleichweit entfernt.

Ruthe des Männchens ohne Penisknochen. Krallen sehr stark und kräftig. Ueber den Augen sind kleine Drüsen bemerkbar. Auf den Lippen und dem Kinn stehen lange, ziemlich starre Haare.

Gebiss: $\frac{1 \cdot 1 \cdot 3 \cdot 2}{2 \cdot 1 \cdot 3 \cdot 3}$. Nur zwei von einander und von den Eckzähnen getrennte Schneidezähne im Oberkiefer. Obere Eckzähne auf der Vorderseite gefurcht. Untere Eckzähne schief nach aussen gerichtet.

Das Männchen hat nach Jentink keine Schulterdrüsen. Thomas sagt (Ann. Mag. Nat. Hist. 1896 (6) XVIII p. 242): ,sides of neck with dark golden gland-tufts". Gaurnen mit vier ungetheilten und drei getheilten Querfalten.

Typus: Boneia bidens Jentink.

Hab. Celebes.

Uebersicht der Arten:

Oberer Ohrrand abgerundet, Vorderkopf goldgelb . . . B. bidens.

Oberer Ohrrand scharf zugespitzt, Vorderkopf dunkelbraun B. menadensis.

1. B. bidens Jentink. - 1879. Boneia bidens von Boné, Süd-Celebes, Jentink, Not. Leyd. Mus. I, p. 117 -119. - 1879. Dobson, Rep. Brit. Assoc. 1880, p. 6. - 1897. Trouessart, 1. c. p. 85.

Hab. Boné, Süd-Celebes,

2. B. menadensis Thomas. - 1897. Boneia menadensis von Menado, Nordost-Celebes, Oldf. Thomas, Ann. Mag. Nat. Hist. (6) XVIII, p. 242-243. 1897. Trouessart, 1. c. p. 85.

\section{Hab. Menado, Nordost-Celebes.}




\section{Genus. Harpyionycteris Thomas.}

1896. Harpyionycteris, Thomas, Ann. Mag. Nat. Hist. (6), XVIII, p. 243-244. - 1898. Thomas, Trans. Z. S. London XIV, 6, p. 384-385, Taf. XXX, Fig. 1 (Thier), XXXV, Fig. 1-4 (Schädel).

Ohne Schwanz. Der Zeigefinger trägt eine Kralle. Der Rand des Ohres bildet, wie bei Pteropus und Xantharpyia einen ovalen Ring. Die Flughaut setzt sich in der Mitte zwischen den Metatarsalien der ersten und zweiten Zehe an. Der Metacarpus des Mittelfingers ist wenig kürzer als der Zeigefinger mit der Kralle. Die Ränder der Nasenlöcher sind nicht röhrenförmig verlängert. Gebiss: $\frac{1.1 .3 .2}{1.1 .3 .3}$ Nur zwei Schneidezähne im Oberkiefer, welche sowohl einander, als auch die Eckżähne berühren. Eckzähne mit Nebenzacken; nach aussen gerichtet. Molaren vielhöckerig.

Typus: Harpyionycteris whiteheadi Thomas.

Hab. Mindoro, Philippinen. $1524 \mathrm{~m}$ hoch.

Nur eine Art: H. whiteheadi Thomas. - 1896. Harpyionycteris whiteheadi von Mindoro, Philippinen, aus der Höhe von 1524 Metern, Oldf. Thomas, Ann. Mag. Nat. Hist. (6), XVIII, p. 244. - 1897. Trouessart, l. c. p. 85. - 1898. Thomas, Trans. Z. S. London XIV. 6, p. 384-385, Taf. XXX, Fig. 1 (Thier), Tafel XXXV, Fig. 1-4 (Schädel).

Ich habe diese Gattung nicht untersuchen können; möchte aber fast vermuthen, dass Harpyionycteris in die Nähe von Styloctenium und Pteropus zu stellen ist und sich zu Pteralopex ähnlich verhält, wie Styloctenium zu Sericonycteris.

\section{\%. Genus. Scotonycteris Matschie.}

1894. Scotonycteris, Matschie, Sitzb. Ges. naturf. Fr. Berlin, p. 200-203.

Ohne deutlichen Schwanz. Der Zeigefinger trägt eine Kralle. Der Rand des Ohres bildet einen ovalen Ring. Die Flughaut heftet sich an den Rücken der ersten Zehe. Der Metacarpus des Mittelfingers ist etwas kürzer als der Zeigefinger mit der Kralle. Die Ränder der Nasenlöcher sind wulstig; Gebeiss: $\frac{2.1 .1 .2}{2.1 .2 .3}$. Der letzte Molar ist in beiden Kiefern im Querschnitt rund und bedeutend kleiner als der vor ihm stehende Molar. Die Caninen sind fangzahnförmig gekrümmt. Vier Schneidezähne in jedem Kiefer, die oberen stehen bogenförmig. Die Backzähne sind denen von Cynopterus ähnlich, aber mehr abgerundet. $Z$ wischen den Eckzähnen und den Praemolaren ein Diastema. Der knöcherne Gaumen reicht weit über die Molarenreihe hinaus. 
Von den Cynopterus-Arten mit undeutlichem Schwanze unterscheidet sich Scotonycteris ausser durch das Gebiss noch dadurch, dass die Schwanzflughaut nicht verkümmert ist.

\section{Hab. Nieder Guinea.}

Nur eine Art: Sc. zenkeri Matschie. - 1894. Scotonycteris zenkeri von der Yaunde Station, Süd-Kamerun, Matschie, Sitzb. Ges. naturf. Fr. Berlin, p. 202-203. - 1897. Sjöstedt, Bih. T. K. Svenska Vet. Akad. Handl. Bd. 23. Afd. IV Nr. 1, p. 46. - 1897. Trouessart 1. c. p. 87.

Hab. Kamerun (Yaude und Elefanten See).

Abbildung auf Tafel [12] (Thier, Flughaut, Kopf) eines jüngeren . Ot, Gaumen und Schädẹls eines alten ㅇ).

[B. M.] \& ad. Yaunde, Süd-Kamerun, Zenker; $\lesssim$ jun. Elefanten-See, Nordwest-Kamerun, Conradt.

Die Anordnung der Gaumenfalten ist bei dem ot jun. etwas anders als beim 우 nämlich die 6. Falte ist nur an den Seiten und in der Hitte angedeutet, die 7. Falte aber in der Hitte getheilt und in zwei stark nach vorn gebogene gezähnelte Hälften getrennt. Auch die Maasse sind bei diesem Exemplar wesentlich abweichend von denen des 우.

\section{Genus. Cynopterus F. Cuv.}

1825. F. Cuvier Dents des Mammif. p .39 und 248. - 1828. Pachysoma, Geoffroy St. Hilaire, Dict. class. V. XIV., p. 704-705. - 1869. Pachysoma partim Fitzinger, Sitzb. Akad. Wien IX. 1. Abth. November, p. 24-46. - 1878. Cynopterus partim Dobson, Cat. Chir. p. 80-86. - 1881. Cynopterus, J. Anderson, Cat. Mamm. Indian Museum I, p. 104-106. - 1887. Cynopterus partim Jentink, Mus. d'Hist. Nat. Pays-Bas, IX, Cat. Osteol. p. 264-266. - 1888. Jentink, 1. c. XII. Cat. Syst. p. 153-155. - 1897. Trouessart, Cat. Mamm. Nov. Ed. I. p. 85-86.

Grosse Fledermäuse entweder mit wohl entwickeltem Schwanz, der zum Theil mit der breiten Schwanzflughant verwachsen ist, an seinem unteren Ende aber so von ihr umschlossen wird, dass er fast vollständig in sie zurückgezogen werden kann, oder ohne deutlichen Schwanz. Der Zeigefinger trägt eine Kralle. Der Rand des Ohres bildet einen ovalen Ring, wie bei den vorhergehenden Gattungen. Die Flughaut setzt sich an die erste Zehe an. Der Metacarpus des Mittelfingers ist bald länger, bald kürzer als der Zeigefinger ohne Kralle. Die Ränder der Nasenlöcher sind wulstig nmrandet, aber nicht röhrenförmig verlängert. Die Schnauze ist kurz. Ueber die Lippe verläuft von der Mitte der Nasenscheidewand geradlinig bis zum Lippenrande eine seichte, jederseits von einem breiten, nackten Wulste begleitete Furche. Gebiss: $\frac{2.1 .2 .2}{2.1 .2 .3}$. Der letzte untere rechte Molar fehlt bei einem mir bekannten Individuum. Ich glaube, dass Cynonycteris grandidieri auf einen recht alten Cynopterus begründet ist, bei welchem der letzte Molar sowohl im Ober- als im Unterkiefer bis auf die Wurzeln abgekaut ist. Auch 
von den Incisiven kann einer oder der andere fehlen, jedoch ist dann stets die Stelle, wo der Zahn gesessen hat, durch eine Lücke angedeutet. - Die Praemaxillaria sind vorn durch Naht verbunden. Die oberen Schneidezähne stehen in einer geraden Linie dicht neben einander und sind von den Eckzähnen durch eine Lücke getrennt, die breiter ist als der äussere Incisivus. Die Backenzähne sind bei jüngeren Thieren stumpfhöckerig mit einer Längsfurche auf der Krone und kauen sich mit dem Alter flach ab. Zwischen dem Eckzahn und den Praemolaren ist keine grössere Lücke. Der knöcherne Gaumen reicht weit über die Molarenreihe hinaus. Auf dem Gaumen stehen 8-12 stark nach hinten gekrümmte Querfalten, von denen die letzten in der Mitte getheilt sind. Am Hinterrande des Gaumens stehen ausserdem noch 3 in der Mitte getheilte, gezähnelte, gerade Querfalten hintereinander. Das Plagiopatagium (die Ellenbogen-Flughaut) zeigt eine ähnliche Vertheilung der Fascien wie bei Epomophorus s. str. - Die Saugwarzen befinden sich an den Seiten der Brust etwas unterhalb der Achselgrube. Nach Dobson und Peters (Monatsb. Akad. Berlin, 1869, p. 395) haben die o zuweilen wohl entwickelte Zitzen, was darauf schliessen lässt, dass sie sich in gewissen Fällen an dem Nähren der Jungen betheiligen. Die Ruthe des Männchens enthält keinen Penisknochen. Zur Fortpflanzungszeit haben die Männchen auf jeder Schulter einen Wirbel von langen, lebhaft gefärbten und ziemlich starren Haaren, welche mit zahlreichen, ein öliges Sekret absondernden Hautdrüsen in Verbindung stehen.

Sonst sind die Cynopterus im allgemeinen ziemlich düster gefärbt, auf dem Rücken mehr bräunlich, auf der Unterseite mehr grau.

Die Kurzkopf-Flederhunde sind Nachtthiere; sie ruhen gesellschaftlich bei Tage auf Bäumen, fliegen geschickt und leicht, haben eine scharfe laute Stimme, fress,en Früchte und sind durch ihre ausserordentliche Gefrässigkeit für die Plantagenwirthschaft sehr schädlich. Sie verbreiten einen eigenthümlichen, unangenehmen Geruch.

Es sind bis jetzt 20 hierher gehörige Species beschrieben worden; von diesen hat Trouessart 6 Arten angenommen.

Drei von den Species C. blanfordi, latidens und melanocephalus unterscheiden sich von den übrigen, sehr nahe mit einander verwandten Formen dadurch, dass bei ihnen der Schwanz nicht zu erkennen, die Schwanzflughaut nur wenig entwickelt und der Fuss länger als das Ohr ist.

Diese 3 Arten vereinige ich einer besonderen Untergattung, für welche ich den

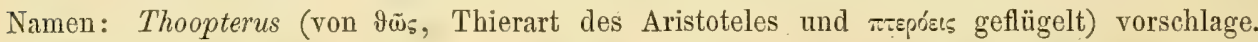
Thomas hat 1893 einen Cynopterus maculatus beschrieben, welcher sich durch seine Zahnformel von allen anderen Cynopterus unterscheidet. Ich halte es für praktisch, Cynopterus maculatus Thos. als Vertreter einer besonderen Gattung Balionycteris (von ßánıos, gefleckt v'stepts Fledermaus) aufzufassen.

Pachysoma giganteum Fitzinger (Zelebor. Säugethiere der Novara Exped. Sitzb. Akad. Wien XLII p. 390; Pt. edulis var.' b. Zelebor. Reise Novara I p. 11 und Pachysoma giganteum Fitzinger, Sitzb. Akad. Wien LX 1. Abth. November p..25) ist sicher kein Cynopterus, sondern wahrscheinlich ein Pteropus nicobaricus.

Hab. Vorder-Indien, nach Westen bis Sind, Hinter-Indien, Sumatra, Java, Borneo, Philippinen, Celebes, Sanghir-Inseln, Soela-Inseln, Timor; unsichere Fundorte: Zanzibar (Cynonycteris grandidieri), Aru-Inseln (Berl. Mus.); Neu-Guinea (P. Z. S. 1875, p. 875). 


\section{Uebersicht der Untergattungen.}

Schwanz deutlich; Schwanzflughaut breit; Ohr länger als Fuss:

Schwanz undeutlich; Schwanzflughaut sehr schmal; Ohr kürzer als Fuss:

Cynopterus F. Cuv. p. 74

Thoopterus Mtsch. p. 77

\section{Uebersicht der geographischen Verbreitung.}

Es ist mir bisher nicht gelungen, den Beweis dafür zu gewinnen, dass man bei Cynopterus s. str. geographische Abarten unterscheiden muss. Entweder giebt es nur eine einzige Form in dieser Untergattung, oder aber sehr viele Abarten, welche auf kleine Gebiete beschränkt sind. Wir haben von Ceylon 2 Exemplare mit kurzen Ohren und ohne Vorsprung am unteren Ohrrande. Unser Nicobaren-Exemplar ist den Ceylonesen sehr ähnlich, hat aber einen sehr kleinen Vorsprung am unteren Ohrrande. Von unseren Stücken aus Vorderindien hat das einzige, welches in Alcohol conservirt ist, sehr lange Ohren und keine Ohrrandausbuchtung; unter den trocken praeparirten Exemplaren sind sowohl kurzohrige als auch langohrige. Am Ohrrande ist ein Vorsprung nicht zu entdecken. Die Stücke von Banka und Sumatra haben einen sehr deutlichen Vorsprung am Ohre und kleine Ohren; bei solchen von Borneo ist der Vorsprung sehr klein und die Ohren sind klein. Bei den Javanern ist der Vorsprung des Ohrrandes nicht deutlich, wohl aber bei den Exemplaren von den Philippinen. -

Jentink hat (Not. Leyd. Mus. XIII, 1891 p. 202-204) Unterschiede zwischen Cyn. brachyotis und Cyn. marginatus resp. titthaecheilus angegeben.

"C. brachyotis at a glance is distinguished from C. marginatus by its white fingers, strikingly contrasting whith the dark wing-membrans, smaller ears, more elongate muzzle and smaller head."

Ferner glaubt Jentink, dass bei brachyotis auf dem Rücken ein „more brownish tinge" vorherrscht, während er bei marginatus "somewhat black" ist. Auch soll das Halsband bei den "brachyotis"-Männchen viel mehr entwickelt sein als bei marginatus.

Herr Jentink giebt einẹ vergleichende Maasstabelle beider Formen.

Unser Material spricht nicht für die Beständigkeit der hier angegebenen Unterschiede. Wenigstens haben wir ein Stück welches nach den Maassen zu brachyotis gehört, aber keine weissen Finger und sehr dunkle Flughäute hat. Dasselbe stammt von Sumatra. Ein anderes von Java, den Maassen nach zu marginatus gehörig, hat weisse Finger und helle Flughänte.

Ich habe in der Verbreitungstabelle die zahlreichen Namen für geographische Formen von Cynopterus s. str. aufgezählt, trotzdem vielleicht alle diese Abarten keine systematische Berechtigung haben; ich thue es doch, weil möglicherweise noch gewisse unterscheidende IIerkmale für dieselben nach der Untersuchung eines reicheren Materials gefunden werden könnten.

Wie sich Th. melanocephalus zu blanfordi verhält, muss durch direkte Vergleichung festgestellt werden. Th. nigrescens unterscheidet sich durch die breiten Molaren von den beiden anderen Formen der Gruppe. 


\begin{tabular}{|c|c|c|}
\hline & Cynopterus & Thoopterus \\
\hline $\begin{array}{l}\text { (?) Zanzibar } \\
\text { Ceylon } \\
\text { Süd-Indien } \\
\text { Hindustan } \\
\text { Hinter-Indien } \\
\text { Nikobaren } \\
\text { Andamanen } \\
\text { Sumatra } \\
\text { Java } \\
\text { Borneo } \\
\text { Philippinen } \\
\text { Sanghir } \\
\text { Soela } \\
\text { Timor } \\
\text { Celebes } \\
\text { Morotay } \\
\text { (?) Neu-Guinea } \\
\text { (?) Aru }\end{array}$ & $\begin{array}{c}\text { grandidieri } \\
\text { ceylonensis } \\
\text { sphinx } \\
\text { marginatus } \\
\text { montani } \\
\text { scherzeri } \\
\text { brachysoma und andamanensis } \\
\text { titthaecheilus } \\
\text { horsfieldii } \\
\text { brachyotis } \\
\text { luzoniensis } \\
\text { als marginatus } \\
\text { do. } \\
\text { do. } \\
\text { marginatus } \\
\text { - } \\
\text { als scherzeri } \\
\text { als scherzeri }\end{array}$ & $\begin{array}{c}- \\
- \\
- \\
\text { blanfordi } \\
- \\
- \\
- \\
\text { melanocephalus } \\
\text { - } \\
\text { nigrescens } \\
\text { - } \\
\text { - } \\
\text { - } \\
\text { nigrescens } \\
\text { nigrescens } \\
\text { - } \\
\text { - }\end{array}$ \\
\hline
\end{tabular}

\section{Subgen. Cynopterus F. Cuv.}

1825. F. Cuvier, Dents des Mammif. p. 39 und 248.

Schwanz deutlich; Schwanzflughaut wohl entwickelt uud breit; das Ohr länger als der Fuss. Unterschenkel nicht bis zur Fusswurzel dicht und lang behaart.

Typus: Cynopterus sphinx (Vahl).

Hab. Vorder-Indien, nach Westen bis Sind, Hinter-Indien, Sumatra, Java, Borneo, Philippinen, Celebes, Sanghir-Inseln, Soela-Inseln, Timor. Unsichere Fundorte: Zanzibar, Aru-Inseln, Neu-Guinea.

1. Cyn. sphinx (Vahl). - 1797. Vespertilio sphinx Vahl von Tranquebar, Scrivter af Naturhistorie-Selskabet, 4de Bind, 1ste Hefte p. 123-126. 1878. Cynopterus marginatus, scherzeri, brachysoma Dobson, l. c. p. 82-86. 1887. Cyn. marginatus Jentink, 1. c. p. 264-266. - 1888. Jentink 1. c. p. 153 -154. - 1890. Jentink, Weber's Zool. Erg. I, p. 126. - 1890. Weber, 1. c. p. 96-98. - 1897. Cyn. marginatus, scherzeri, brachysoma, montanoi, Trouessart, 1. c. p. $85-86$.

Hab. Verbreitung wie bei der Untergattung.

Ich zähle nunmehr die bisher beschriebenen Lokalformen in geographischer Reihenfolge auf. Vielleicht werden alle unter einem einzigen Namen vereinigt werden müssen.

1. Cyn. ceylonensis Gray. - 1870. Cynopterus marginatus var. ceylonensis Gray von Ceylon, Cat. Monk. Lem. Fruit-eating Bats, p. 122.

Hab. Ceylon. 
2. Cyn. sphinx (Vahl). - 1797. Vespertilio sphinx Vahl von Tranquebar, 1. c. p. 123-126. - 1870. Cynopterus marginatus var. Elliotii Gray von Madras, 1. c. p. 122. - 1898. partim F. de Seabra, Jorn. Lisboa, p. 170, Taf. I, Fig. 16 (Gaumen).

Hab. Südl. Vorder-Indien.

3. Cyu. marginatus (F. Cuv.). - 1810. Pteropus marginatus Geoffr. von Bengalen, Ann. Mus. XV p. 97, Tafel 8 (Thier). - 1869. Cyn. marginatus Fitzinger, 1. c. p. 36-39.

Hab. Nördl. Vorder-Indien.

4. Cyn. Scherzeri Fitzinger. - 1861. Pachysoma scherzeri Fitzinger von Kar-Nikobar, Sitzb. Akad. Wien XLII, p. 390. - 1868. Zelebor, Reise Novara, Säugeth. p. 13. - 1869. Fitzinger 1. c. p. 27-29. - 1873. Dobson, Journ. As. Soc. Beng. p. 201, Taf. XIV, Fig. 6 (Ohr). - 1878. Dobson 1. c. p. $84-85$. - (?) 1878. Dobson, P. Z. S. p. 875. - 1879. Trouessart, 1. c. p. 86 .

Hab. Nikobaren (wird von Dobson p. 875 für Neu-Guinea erwähnt).

5. Cyn. andamanensis Dobs. - 1873. Cynopterus marginatus var. andamanensis Dobson von den Andamanen, Dobson, Journ. As. Soc. Beng. p. 201, Taf. XIV, Fig. 5 (Ohr). - 1876. Cyn. brachyotus Dobson, Monogr. Asiat. Chir. p. 26. - 1881. Cyn. andamanensis Anderson, Cat. Mamm. Ind. Mus. I, p. 105.

Hab. Andamanen.

6. Cyn. brachysoma Dobs. - 1871. Cynopterus brachysoma Dobson von den südlichen Andamanen, Journ. As. Soc. Beng. p. 260. - 1873. Dobson 1. c. p. 202, Taf. XIV, Fig. 7 (Ohr): - 1878. Dobson, Cat. Chir. p. 85-86. - 1897. Trouessart, 1. c. p. 86.

Hab. Südl. Andamanen.

7. Cyn. montani Robin. - 1881. Cynopterus montani Robin von Kessang auf Malakka, Bull. Soc. Philom. V, p. 90. — 1891. Robin, Ann. Sc. Nat. Zool. XIII, Art. 2, p. 1-3, Tafel 12, Fig. 2 (Interfemoral-Membran), Fig. 3, (die ersten drei Finger), Fig. 4 (Ohr). - 1881. Robin. Biblioth. Hautes Etudes, XXVI, p. 1, Taf. 1. (Nicht gesehen.) - (?) 1886. C. marginatus Thomas, P. Z. S. p. 73. - (?) 1892. Thomas, An. Mus. Genova, 2. ser., X, p. 921. - (?) 1894. Thomas, Nov. Zool. p. 655. - 1897. C. montano Trouessart, 1. c. p. 86. - 1898. Cynopterus angulatus Gerrit S. Miller jr. von Trong, Unter-Siam, Proc. Acad. Philadelphia, p. 316-319.

Hab. Malakka [Kessang; Salangore, Singapore (Thomas 1886); Trong].

8. Cyn. titthaecheilus (Temm.). -- 1827. Pteropus titthaecheilus T'emminck von Sumatra, Java und Siam, Monogi. Mamm. I, p. 198-200, 261, Taf. 15, Fig. 17--24 (Schädel von o und 우 ad und juv.). - 1828. Pachysoma diardi Geoffroy St. Hilaire von Sumatra, Cours d'hist. nat. Mamm. I. Lec. 13. p. 27. - 1828. P. duvaucelii Geoffroy St. Hilaire von Sumatra. 1. c. p. 28. - 1828. P. brevicaudatum Js. Geoffroy St. Hilaire von Sumatra, 
Ann. Sc. Nat. Zool. XV, p. 204-205. - 1869. Fitzinger 1. c. p. 29-33, 34-36, 39-43. - 1883. C. marginatus Jentink, Not. Leyd. Mus. V. p. 173. - 1889. Thomas, An. Mus. Genova, 2. ser. VII. p. $241-242$ - 1895. Thomas, 1. c. XIV p. 664. - 1898. C. marginatus partim F. de Seabra Jorn. Lisboa, p. 170.

Hab. Sumatra [Silago, Benkoelen, Solok, Priaman, Palembang, Padang], Nias, Mentawei (Thomas).

9. Cyn. horsfieldii Gray. - 1843. Cynopterus Horsfieldii Gray von Java, List Mamm. Brit. Mus. p. 38. - 1869. Fitzinger 1. c. p. 33-34. - 1870. Gray, Cat. Monk. Lem. etc. p. 123.

Hab. Java [Tjikao].

10. Cyn. brachyotis (S. Müll.). - 1839. Pachysoma brachyotis von Borneo, S. Müller, Tijdschrift vor natuurlijke Geschiedenis en Physiologie, herausgegeben von Van der Hoeven, V, p. 146. - 1869. Fitzinger, l. c. p. 43-44. - 1876. Dobson, Monogr. Asiat Chiropt. p. 26. - 1887. Jentink, l. c. p. 266. - 1888. Jentink, I. c. p. 154. - 1893. Everett, P. Z. S. p. 494. - 1894. Thomas, Nóv. Zool. I, p. 656. - 1897. Jentink, Not. Leyd. Mus. XIX, p. 49,50 .

Hab. Borneo [Dewej-Flnss, Sintang].

11. Cyn. Iuzoniensis (Ptrs.). - 1861. Pachysoma Luzoniense Peters vom Vulkan Yriga, Südcamarines, Luzon, Monatsb. Akad. Berlin, p. 708. - (?) 1870. Cynopterus marginatus var. philippensis und var. Cumingii von den Philippinen, Gray, Cat. Monk. Lem. etc. p. 123. - 1890. C. marginatus, Steere, List Birds ands Mammals, p. 28. - 1895. Casto de Elera, Cat. Fauna Filipinas I, p. 7. - 1896. Elliot, Field Columbian Museum, Zool. Ser. vol. 1 Nr. 3 p. 79.

Hab. Philippinen.

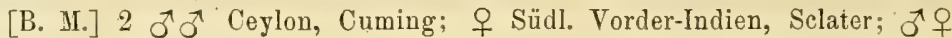
Coorg, Lord Walden; o 3 우 $q$ Bengalen, Umlauff und Lamare Piquot; 우 Nicobaren, Parreis; $0^{\star}$, 우우 pull. Banka, von Martens; 우 Mergui, Philippi; ‥ juv. Perak, Hartert; ơ Deli, Sumatra, Jlartin; 2 우우 Lahat, Sumatra, Schneider und Dietz; \& Sumatra, Leydener Museum, wahrscheinlich von Diard und Duvaucel gesammelt; ơ Ajer mantang, Sumatra, Beccari; 오 Sumatra, Petersen; 우 juv. Sintang, Borneo, von Martens; 오 Sarawak, Borneo durch Marquis Doria; $0^{-1}, 2$ 우 아 Java, Leydener Museum und Dr. Meyer;

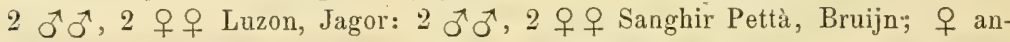
geblich Aru-Inseln; 2 우 a aus der Anatomischen Sammlung ohne Fundortsangabe.

12. Cyn. grandidieri Peters. - 1869. Cynonycteris Grandidieri Peters von Zanzibar, Peters, Monatsb. Akad. Berlin, p. 394-395. - 1878. Dobson, 1. c. p. 79. - 1897. Trouessart, 1. c. p. 85.

\section{Hab. Zanzibar,}

Abbildung des Schädels nach dem in Paris befindlichen Original-Exemplare, einem ơ, auf Tafel [8] Fig. 4. 
2. Subgen. Thoopterus Mtsch. subgen. nov.

Schwanz nicht deutlich; Schwanzflughaut sehr wenig entwickelt und von den Haareu zum grössten Theile verdeckt; das Ohr ist kürzer als der Fuss. Dér Unterschenkel ist bis zur Fusswurzel dicht und lang behaart.

Typus: Cynopterus nigrescens Gray.

Hab. Hinter-Indien, Java, Celebes, Morotai, Philippinen (?).

Uebersicht der Arten:

Unterarm bei erwachsenen Thieren mindestens $65 \mathrm{~mm}$ lang: . . . . . . . . Cyn. nigrescens.

Unterarm höchstens $60 \mathrm{~mm}$ lang: . . . Cyn. melanocephalus und Cyn. blanfordi.

1. Cyn. nigrescens Gray. - 1870. Cynopterus marginatus var. nigrescens Gray von Morty Island, Cat. Monk. Lem. Fruit-eating Bats p. 123. - 1878. Cynopterus latidens Dobson von Morty Island, Cat. Chiroptera, p. 86, Taf. V, Fig. 3 (Gebiss). - 1888. Jentink, 1. c. p. 155. - 1895. Casto de Elera, Cat. Syst. Faune Filipinas, I p. 7. - 1897. Trouessart l. c. p. 86. (nach Elera).

Hab. Morotai (Halmahera-Gruppe); Celebes; Laguna, Batangas auf Luzon [B. M.] \& Menado, Celebes, von Faber.

2. Cyn. melanocephalus Temm. - 1827. Pteropus melanocephalus Temminck von Bantam auf Java, Monogr. Mamm. I, p. 190-191 Taf. XII (Thier) Taf. 16, Fig. 3 (Skelett), Fig. 4 (Gebiss von vorn). - 1837. 'Temminck, 1. c. p. 82, 92, Taf. 35, Fig. 10 (Kopf). - 1869. Pachysoma melanocephalum Fitzinger, 1. c. p. $45-46$. - 1878. Cyn. melanocephalus Dobson, 1. c. p. 85. 1887. Jentink, Cat. Ost. p. 266. - 1888. Jentink, Cat. Syst. p. 155. 1893. Thomas, Ann. Mag. Nat. Hist. vol. XI. ser. 6 p. 342. - 1897. Trouessart, l. c. p. 87.

Hab. Java: Bantam.

3. Cyn. blanfordi Thos. - 1891. Cynopterus blanfordi Thomas von Leitò, Cheba in den Carin Hills, Ann. Mus. Genova 2. ser. XXX p. 884. - 1892. Thomas, 1. c. p. 921-922, Taf. XI, Fig. 1. (Interfemoral-Membran) und Fig. 2 (Ohr). - 1897. Trouessart, l. c. p. 86.

Hab. Birma: (Leitò, Cheba, Carinn-Hills.)

\section{Genus. Ptenochirus Peters.}

1861 Ptenochirus Peters, Monatsb. Akad. Berlin, p. 707. - 1878. Dobson 1. c. p. 87. - 1897. Trouessart, 1. c. p. 86 .

Aehnlich Cynopterus; Zeigefinger mit. Kralle. Der Rand des Ohres bildet einen ovalen Ring. Die. Flughaut setzt sich an die erste Zehe an. Der Metacarpus 
des Mittelfingers ist kürzer als der Zeigefinger mit der Kralle. Nasenlöcher und Oberlippe wie bei Cynopterus. Gebiss: $\frac{2.1 .22}{1.1 .2 .3}$. Die Praemaxillaria sind vorn durch Naht verbunden. Die oberen Schneidezähne stehen in einer geraden Linie dicht neben einander und sind. von den Eckzähnen durch eine Lücke getrennt, die noch nicht so breit ist, wie der äussere Incisivus. Die inneren Schneidezähne sind mindestens um die Hälfte länger als die äusseren. Backenzähne ähnlich wie bei Cynopterus. Zwischen dem Ėckzahn und den Praemolaren ist keine grössere Lücke. Der knöcherne Gaumen reicht weit über die Molarenreihe hinaus. Die Ruthe des Männchens enthält keinen Penisknochen. Die Gaumenfalten, die Saugwarzen, das Auftreten von Schulterbüscheln, die Vertheilung der Fascien der Flughaut und die allgemeine Färbung erinnert sehr an die gleichen Verhältnisse bei Cynopterus:

Ueber die Lebensweise der hierher gehörigen Arten weiss ich nichts.

Hab. Philippinen, Borneo, Sumatra.

\section{Uebersicht der Untergattungen.}

Schwanz deutlich; die Flughaut setzt an die Basis der ersten Phalaux des Hallux an . . . . . . . . . . . . . . . . . Ptenochirus Ptrs. Schwanz fehlt; die Flughaut setzt sich an das distale Drittel der ersten Phalanx des Hallux an . . . . . . . . . . . . . Megaerops Ptrs.

Uebersicht der geographischen Verbreitung.

\begin{tabular}{c|c|c} 
& Ptenochirus. & Megaerops. \\
\hline $\begin{array}{c}\text { Philippinen } \\
\text { Borneo } \\
\text { Sumatra }\end{array}$ & jagori & $\begin{array}{l}\text { ecaudatus } \\
\text { ecaudatus }\end{array}$ \\
\hline
\end{tabular}

\section{Subgen. Ptenochirus Peters.}

1861. Peters, Monatsb. Akad. Berlin, p. 707.

Die Flughant setzt sich an die Basis der ersten Phalanx des Hallux an; der Schwanz ist dentlich, die Schwanzflughaut wohl entwickelt und breit; das Ohr ist ungefähr so lang wie der Fuss mit den Krallen; der Unterschenkel ist kurz behaart.

Typus: Ptenochirus jagori Ptrs.

Hab. Philippinen, Borneo, Malakka.

Uebersicht der Arten:

Unterarm länger als $70 \mathrm{~mm}$. . . . . . . . . . . . Pt. jagori Ptrs.

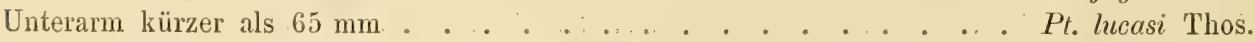


1. Pt. jagori Peters. - 1861. Pachysoma (Ptenochirus) Jagorii Peters von Daraga, Provinz Albay, Luzon, Monats. Akad. Berlin, p. 707-708. - 1878. Dobson, l. c. p. 87. - 1887. Hoffmann, Abh. Mus. Dresden 1886/87 p. 24 (Beschreibung eines alten 우). - 1895. Casto de Elera, Cat. Sist. p. 7. - 1897. Trouessart l. c. p. 87 .

Abbildung des Thieres, seines Kopfes, Gaumens und Schädels auf Tafel [13].

Hab. Philippinen [Luzon, Mindoro, Süd-Mindanao (Hoffmann 1887)].

[B M.] ơ Daraga, Luzon, Jagor; $\widehat{0}$ Caminguin, Luzon, Semper, 우 Calapan, Mindoro, Platen.

2. Pt. Iucasi Dobson. - 1880. Cynopterus (Ptenochirus) Lucasii Dobson von Sarawak auf Borneo, Ann. Mag. Nat. Hist. ser. 5, vol. VI p. 163-164. 1893. Everett, Proc. Zool. Soc. London, p. 494. - 1897. Trouessart, 1. c. p. 86. - 1897. Jentink, Not. Leyd. Mus. XIX, p. 50.

Hab. Borneo (Sarawak, Dobson; Roema Manoeal am Kenepai und Goenong Dadap, westlich davon, Jentink).

\section{Subgen. Megaerops Peters.}

1841. Megaera Temminck, Monogr. Mamm. II, p. 359. - 1867. Megaerops Peters, Monatsb. Akad. Berlin, p. 867. - 1869. Cynopterus Fitzinger, nec Cuvier, Sitzb. Akad. Wien LX, 1. Abth. November, p. 46.

Die Flughaut setzt sich an das distale Drittel der ersten Phalanx des Hallux an; der Schwanz ist nicht sichtbar, die Schwanzflughaut an den Seiten wohl entwickelt, das Ohr ist länger als der Fuss mit den Krallen; der Unterschenkel ist kurz behaart. - S. Müller fand diese Gattung auf. Felsen am Strande von Padang, Sumatra.

Typus: Meg. ecaudatus Temm.

Hab. Sumatra, Borneo.

Nur eine Art: M. ecaudatus Temminck. - 1837. Pachysoma ecaudatum Temminck von Padang auf Sumatra, Monogr. Mammal. II. p. 94-95. - 1841. Megaera ecaudata Temminck, l. c. II, p. 357-359, Taf. 69 (Thier, Kopf, Schädel, Gebiss). - 1863. Macklot und Müller, Mém. Quadrum. Chiropt. del' Arch. Ind. p. 117. - 1867. Megaerops ecaudatus Peters, Monatsb. Akad. Berlin, p. 868. - 1869. Cynopterus ecaudatus Fitzinger, Sitzb. Akad. Wien LX, 1. Abth. November, p. 47-48. - 1870. Megaera ecaudata Gray, Cat. Monk. Lem. etc., p. 124. 1878. Megaerops ecaudatus Dobson, 1. c. p. 87. - 1887. Cynopterus ecaudatus Jentink, Cat. Ost. p. 266. - 1888. Jentink, Cat. Syst. p. 155. - 1889. Thomas vom Kina Balu in Nord-Borneo, Proc. Zool. Soc. London, p. 229. (Unterschiede von C. lucasi). - 1893. Everett, Proc. Zool. Soc. London, p. 494. 1897. Megaerops ecaudatus Trouessart, 1. c. p. 86. - 1897. Cyn. ecaudatus Jentink, Not. Leyd. Mus. XIX, p. 50-51 (Maasse). - 1898. F. de Seabra, Jorn. Scienc. Math. Phys. Nat. Lisboa, 2. ser. V, p. 170.

Abbildung des Schädels nach dem Leydener Original-Exemplare auf Taf. [8], Fig. 5.

(Jentink erwähnt 1887 diesen Schädel mit der Bemerknng: "Hanquent les mâchoires inférieures et les arcs zygomatiques." Als Peters 1867 den Schädel 
zeichnen liess, war der Unterkiefer noch vorhanden, wie die Abbildung beweist; Temminck's Tafel wird so wesentlich ergänzt.)

Hab. Sumatra (Padang, Temminck), Borneo (Kina Balu, Thomas; Smitan am Kapuas-Fluss, Jentink; Lah Datu, nabe der Darvel Bay, Berl. Mus.)

[B. M.] $\sigma^{\pi}$ ohne genauen Fundort, Umlauff; 오 Darvel Bay. Lah Datu, Pagel.

Das Original-Exemplar hat nach Peters (1867) einen Unterarm von $55 \mathrm{~mm}$ Länge, bei dem Exemplare vom Kapuas ist nach Jentink (1897) der Unterarm $58 \mathrm{~mm}$ lang; ebenso gross ist das o des Berliner Museums. Das ㅇ von Lah Datu hat nur $50 \mathrm{~mm}$ Unterarm-Länge, ist aber, wie die abgekauten Molaren vermuthen lassen, ein ausgewachsenes Thier.

\section{Genus. Balionycteris Matschie gen. nov.}

Wie Megaerops mit 2 unteren Incisiven und ohne Schwanz, aber die inneren oberen Incisiven sind zweimal so lang wie die äusseren und im Oberkiefer stehen jederseits 5 Molaren, von welchen der erste Praemolar und der letzte Molar sehr klein sind.

Ich habe Cynopterus maculatus Thos. als besonderer Gattung aufgefasst, weil diese Art sich durch sehr verschiedene Anzahl und Gestalt der Zähne auszuzeichnen scheint. Thomas vergleicht sie mit C. melanocephalus. Diese Art hat aber, wie Temminck's Abbildung beweist, eine nur wenig entwickelte Schwanzflughaut, während bei $C$. maculatus die „interfemoral membrane fairly well developed" sein soll. Ausserdem hat C. melanocephalus, wie Thomas auch erwähnt, ein ganz anderes Gebiss.

\section{Hab. Borneo.}

Nur eine Art: B. maculata Thomas. - 1892 (?) Cynopterus spadiceus Thomas vom Baram, Nordost-Sarawak, Borneo, nomen nudum, Proc. Zool. Soc. London, p. 227. - 1893. Cynopterus maculatus von Sarawak, Thomas, Ann. Mag. Nat. Hist. ser. 6, vol. XI, p. 341-342. - 1893. C. spadiceus und maculatus, Everett, Proc. Zool. Soc. London, p. 494. - 1897. Cyn. maculatus Thos., Trouessart, l. c. p. 87. - 1897. Jentink, Not. Leyd. Mus. XIX, p. 51.

Hab. Borneo (Baram in Sarawak, Thomas; Kenepai-Berg, Jentink).

Das Exemplar, welches Thomas beschrieb, ein erwachsenes $\widehat{\jmath}$, hat $41 \mathrm{~mm}$ Unterarm-Länge, das von Büttikofer gesammelte, von Jentink erwälnte Stück, ist ein $q$ und hat den Unterarm $37 \mathrm{~mm}$ lang.

Nach Thomas und Jentink sind die zahlreichen gelben Flecke auf den Gelenken und Flughäuten für diese Form bezeichnend. 


\section{Genus. Gelasinus Temminck*}

1810. Cephalotes partim E. Geoffroy St. Hilaire, Ann. Mus. Paris XV, p. 101-108. - 1811. Harpyia Illiger, Prodromus Syst. Mamm. p. 118 (von Ochsenheimer 1810 schon für eine Lepidopteren-Gattung verwendet). - 1828. Cephalotes J. Geoffroy St. Hilaire, Dict. class. d'hist. nat. XIV, p. 706. - 1837. Gelasinus Temminck, Monogr. Mammal. II, p. 100. - 1862. Uronycteris Gray, Proc. Zool. Soc. p. 262. - 1863. Gray, Ann. Mag. Nat. Hist. ser. 3, XI, p. 385-386. 1869. Harpyia Fitzinger, Sitzb. Akad. Wien LX, 1. Abth. November, p. 48-53. - 1878. Dobson 1. c. p. 88-90. - 1892. Uronycteris Douglas Ogilby, Cat. Austr. Mamm. p. 81. - 1898. Cephalotes Palmer, Proc. Biol. Soc. Washington XII, p. $112-114$.

Mittelgrosse Fledermäuse, deren Schwanz fast so lang wie der Unterschenkel und zur Hälfte in die breite Schenkelflughaut eingeschlossen ist, mit einer Kralle am Zeigefinger. Der Rand des Ohres bildet wie bei allen Pteropodiden einen ovalen, geschlossenen Ring. Die Flughaut setzt sich an die Basis der zweiten und dritten Zehe an. Schnauze kurz, dick und breit; die Nasenlöcher liegen in röhrenförmigen Hau.tvorsprüngen; die Lippen sind stark aufgetrieben und auf der Innenseite mit stabförmigen Zotten besetzt. Von der Nase bis zum Lippenrande verläuft eine schmale, tiefe Rinne. Der Metacarpus des Mittelfingers ist ungefähr so lang wie der Zeigefinger mit Kralle; der Daumen ist zur Hälfte von der Flughaut eingehüllt. Die Ohren sind oval und klein, kürzer als die Breite zwischen den Mundwinkeln; die Augen sind gross, die Pupille steht wagerecht und ist spaltförmig. Die Saugwarzen befinden sich an den Brustseiten, etwas unterhalb der Achselgrube. Die Ruthe des Männchens hat keinen Penisknochen. Gebiss: $\frac{2.1 .2 .2}{0.1 .2 .3}$. Die beiden oberen Schneidezähne füllen den Raum zwischen den Eckzähnen aus; die Backenzähne sind ähnlich wie bei Cynopterus. Der erste obere Praemolar ist nur

* Ich kann mich Herrn Palmer nicht anschliessen, wenn er glaubt, dass Geoffroy St. Hilaire 1810 als Typus seiner Gattung Cephalotes den Vespertilio cephalotes Pall. gewählt habe, und dass man deshalb den Namen Cephalotcs für die hier zu besprechende Gattung annehmen müsse. E. Geoffroy St. Hilaire hat die Gattung Cephalotes 1810 aufgestellt und zwei sehr verschiedene Arten in derselben vereinigt, nämlich Cephaloces Peronii (Ann. Mus. XV, p. 104) und Cephalotes pallasii (1. c. p. 107), welche mit Vespertilio cephalotes Pall. synonym ist. Geoffroy hat Vesp. cephalotes Pall. nicht untersucht [je n'en parle que sur le témoignage de Pallas, l. c. p. 107], wohl aber hat er seinen Cephalotes Peronii vor Augen gehabt. Er giebt als Diagnose der Gattung Cephalotes u. a. folgendes an: Dents incisives $\frac{2}{2}-$ canines $\frac{2}{2}-$ molaire $\frac{8}{12}$. Da nun Vesp. cephaotes nur 10 Molaren und keine Incisiven im Unterkiefer besitzt, für C. Peronii Geoffr. dagegen diese Diagnose zutrifft, so muss man als Typus der Gattung Cephalotes die letztere Art festbalten. Geoffroy hat sie überdies an erster Stelle genannt. Wenn dieser Autor später den Namen Cephalotes auf Vesp. cephalotes beschränkt und für die andere Art den neuen Gattungsnamen Hypoderma gegeben hat, so darf man diese Aenderung nicht annehmen, weil die Diagnose von Cephalotes auf Vesp. cephalotes nicht passt. Cephalotes hat 2 untere Incisiven und 12 untere Molaren, folglich kann Vesp. cephalotes nicht zur Gattung Cephalotes Geoffr. gehören. Da Harpyia Ill. später als Harpyia Ochsenheimer aufgestellt worden ist, so muss dieser Name fallen. T'emminck erwähnt 1837 für unsere Gattung den Namen Gelasinus, welchen ich nunmehr hier annehme. 
so gross wie der Basalwulst des zweiten und schliesst sich ohne Diastema an die Canine an. Die unteren Caninen berühren sich fast.

Der Schädel ist kurz und breit, im Nasentheil zusammengedrückt und hoch. Die Praemaxillaria sind mit einander verwachsen. Der knöcherne Gaumen reicht weit über die Molaren-Reihe hinaus. Auf dem Gaumen befinden sich 22-24 Falten, von denen die mittleren stark nach hinten gebogen sind, die vorderen und die hinteren geradlinig verlaufen. Die auf dem Hinterende des Gaumens befindlichen Falten sind gezähnelt und stehen sehr nahe bei einander; die letzten sind in der Mitte unterbrochen und fassen ein ovales nacktes Feld ein.

Auf dem Plagiopatagium (der Ellenbogen-Flughaut) wird der vom Ellenbogen zur Spitze des fünften Fingers verlaufende Strang, welcher sich nach unten in mehrere starke Aeste spaltet, von 15-23 zur freien Kante der Flughaut parallelen Strängen durchschnitten. Der obere Theil der Flughaut ist ebenfalls von parallelen Quersträngen durchzogen.

Die Färbung der Gelasinus-Arten ist braungrau oder röthlichgrau auf dem Rücken, schmutzig weiss auf der Unterseite. Eine dunkle Längsbinde über die Wirbelsäule ist mehr oder weniger deutlich. - Macklot und S. Müller fanden im Magen einer Fledermaus dieser Gattung, welche man „Röhrennasen" nennen könnte, Reste von Käfern und Fliegen.

Drei Arten sind bis jetzt beschrieben worden. Vespertitio cephalotes Pallas von den Molukken, Uronycteris albiventer Gray von Morotai und Harpyia major Dobs. von Neu-Lauenburg; Cephalotes pallasii E. Geoffroy ist Synonym zu V. cephalotes Pallas.

$H$. major unterscheidet sich von $V$. cephalotes Pall., wie unten dargelegt ist, in so wesentlichen Merkmalen, dass ich es vorziehe, diese Art als eine besondere Gruppe ab-

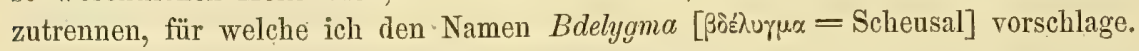

Hab. Celebes, Halmahera- und Amboina-Gruppe, Neu-Guinea, Bismarck-Archipel, Salomons-Inseln, Nord-Australien.

\section{Uebersicht der Untergattungen.}

Die Entfernung zwischen dem vorderen Augenwinkel und der Mitte der auf der Oberlippe vorhandenen Rinne ist grösser als die Breite des Gesichtes an den Mundwinkeln, wenn man die Lippen an den Kiefer andrückt; Unterarm höchstens $70 \mathrm{~mm}$ bei erwachsenen. Stücken, deren Epiphysen an den Fingergelenken mit den Phalangen-Enden verwachsen sind; Unterarm höchstens $40 \mathrm{~mm}$ bei Thieren mit Milchgebiss . . . . . Gelasinus Temm. p. 83.

Das Gesicht ist, wie oben gemessen, breiter als lang; der Unterarm erwachsener Thiere ist mindestens $72 \mathrm{~mm}$ lang, derjenige von jungen Thieren mit Milchgebiss mindestens $45 \mathrm{~mm}$

Bdelygma Mtsch. p. 84 . 
Uebersicht der geographischen Verbreitung.

\begin{tabular}{c|c|c}
\hline & Gelasinus & Bdelygma \\
\hline Celebes & cephalotes & - \\
Halmahera-Gruppe & cephalotes & - \\
Amboina-Gruppe & cephalotes & - \\
Nord-Australien & cephalotes & major \\
Neu-Guinea & cephalotes & major \\
Bismarck-Archipel & cephalotes & major \\
Salomon-Inseln & - &
\end{tabular}

\section{Subgen. Gelasinus Temm.}

1837. Temminck, Monogr. Mamm. II. p. 101.

Die äussere Entfernung der letzten oberen Molaren von einander ist grösser als die Länge der oberen Molarenreihe; der zweite untere Praemolar hat nur einen deutlich ausgebildeten Nebenhöcker. Der Jochbogen ist in der vorderen Hälfte schmäler als in der hinteren. Die Breite des Gesichtes bei den Mundwinkeln (gemessen, nachdem man die Lippen gegen den Kiefer gedrückt hat) ist nicht so gross wie die Entfernung des vorderen. Augenwinkels von der Mitte der Rinne, welche zwischen den Nasenlöchern beginnt und zum Lippenrande verläuft. Das Ohr ist ungefähr so lang wie der Hinterfuss mit den Krallen.

Typus: Vespertilio cephalotes Pall.

\section{Guinea, Bismarck-Archipel.}

Hab. Celebes, Halmahera-Gruppe, Amboina-Gruppe, Nord-Australien, Neu-

Unser Material reicht nicht aus für eine Lösung der Frage, ob es eine oder mehrere Formen in dieser Gruppe giebt. Es scheint, als ob auch hier, wie bei Cynopterus Exemplare mit dunklen Flügeln und solche mit hellen Flügeln in derselben Gegend leben. Ob man es hier vielleicht mit Saisonkleidern zu thun hat, vermag ich vorläufig nicht zu entscheiden. Bei der Aufzählung unseres Materials habe ich in Klammern die Länge des Unterarmes beigefügt.

G. cephalotes (Pallas). - 1767. Vespertilio cephalotes Pallas von den Molukken, Spicileg. Zoolog. III. p. 10-23, Taf. I (Thier und Kopf), Taf. II, Fig. 1 (Skelet), 2-3 (Schädel), 4 (Lippenzotten), 5-6 (Zunge), 7 (Leber), 8-9 (Magen und Milz), 10 (Vagina). - 1810. Cephalotes Pallasii E. Geoffroy St. Hilaire, Ann. Mus. XV, p. 107-108. - 1811. Harpyia cephalotes Illiger, Prodromus Syst. Mamm. p. 118-119. - 1837. Harpyia pallasii Temminck, Monogr. Mamm. II, p. 101-103, Taf. XL, Fig. 1-5 (Thier, Kopf und Schädel). 1862. Cynopterus (Uronycteris) albiventris Gray von Morotai, Proc. Zool. Soc. London, p. 262. - 1863. Harpyia cephalotes Macklot und S. Müller, Mém. Quadrum. Chir. p. 117-121 (Nahrung). - 1869. Harpyia Pallasii Fitzinger, Sitzb. Akad. Wien LX, 1. Abth. November p. 49-52. - 1877. Harpyia cepha- 
lotes Dobson, Proc. Zool. Soc. London, p. 117-118, Fig. 3 (Schädel), 3 a (Unterkiefer). - 1878. Dobson 1. c. p. 88-89. - 1879. Ramsay, Proc. Linn. Soc. New-South Wales, III, p. 243: - 1881. Peters und Doria, Ann. Mus. Civ. Genova p. 690-691. - 1883. Jentink, Not. Leyd. Mus. V, p. 173-174. — 1887. Jentink, Cat. Syst. p. 155 -156. - 1888. Jentink, Cat. Ost. p. 266. 1892. Uronycteris cephalotes Douglas Ogilby, Cat. Austr. Mamm. p. 80-81. 1897. Trouessart, 1. c. p. 87. - 1897. Thomas, Ann. Mus. Civ. Genova, ser. 2, XVIII (XXXVIII) p. 608. - 1897. Heller, Abh. Ber. Zool. Mus. Dresden VI, Nr. 8, p. 4. - 1898. Cephalotes cephalotes (Pall.), Palmer, Proc. Biolog. Soc. Washington, XII, p. 114.

Hab. Celebes, Halmahera-Gruppe, Amboina-Gruppe (Jentink), Nord-NeuGuinea (Arfak, Jentink; Andai und Mansinam, Peters u. Doria), Süd-0st-Neu-Guinea (Milne Bay, Thomas 1897, Heath-Insel, Ramsay 1879), Cap York (Dobson 1878), Neu-Pommern (Berl. Mus.).

[B. M.] $\sigma^{7}$ Manado. Celebes, A. B. Meyer $(67,3 \mathrm{~mm})$; ㅇ Amboina, Beccari $(63 \mathrm{~mm})$; 오 Amboina, Mrüller u. Macklot $(61 \mathrm{~mm})$; 9 pull. Ternate, A. B. Meyer $(30,5 \mathrm{~mm}) ; \sigma^{\top}$ Ghebeh, Bernstein $(55,9 \mathrm{~mm})$; $\sigma^{\nearrow}$ Andai, Nordwest-Neu-Guinea, d'Albertis (56,7 mm); ठ Matupi, Neu-Pommern, Dahl (54,5 mm); ठ Neu-Pommern, Finsch $(54,9 \mathrm{~mm})$; ㅇ juv. Ratavoul, Neu-Pommern, Godeffroy (48,8 mm).

2. Subgen. Bdelygma Mtsch. subgen. nov.

Die äussere Entfernung der letzten oberen MIolaren von einander ist gleich der Länge der oberen Molarenreihe; der zweite untere Praemolar hat zwei deutlich ausgebildete Nebenhöcker. Der Jochbogen ist vorn und hinten ungefähr gleich breit, aber in der Mitte verschmälert. Die Breite des Gesichtes bei den Mundwinkeln (gemessen, nachdem man die Lippen gegen den Kiefer gedrückt hat), ist mindestens so gross, wie die Entfernung des vorderen Augenwinkels von der Mitte der Rinne, welche zwischen den Nasenlöchern beginnt und zum Lippenrande verläuft. Das Ohr ist kürzer als der Hinterfuss mit den Krallen.

Typus: Harpyia major Dobs.

Hab. Misol, Neu-Guinea, Bismarck-Archipel, Salomon-Inseln.

G. major (Dobson). - 1877. Harpyia major Dobson von einer Reise nach NeuLauen burg und den benachbarten Theilen Neu-Pommerns und Neu-Mecklenburgs, Proc. Zool. Soc. London, p. 117-118, Fig. 1 (Kopf), 2 (Schädel), 2 a (Unterkiefer). - 1878. Dobson 1. c. p. 89-90. - 1887. Jentink, Cat. Syst. p. 156. - 1887. Thomas, Proc. Zool. Soc. London, p. 323, 327. - 1888. Jentink, Cat. Ost. p. 267. - 1888. Thomas, Proc. Zool. Soc. London, p. 476, 483. - 1895. Thomas, Nov. Zool. II, p. 163. - 1896. Thomas, l. c. III, p. 526. 1897. Trouessart, 1. c. p. 87. - 1897. Heller, Abh. Ber. Zool. Mus. Dresden VI, Nr. 8, p. 4. - 1898. Cephalotes major (Dobs.), Palmer, Proc. Biolog. Soc. Washington, XII, p. 114.

Hab. Misol (Jentink), Neu-Guinea (südl. vom Huou Golf, Dobson 1878), d'Entrecasteaux-Inseln (Goodenough Island, Fergusson Island, Thomas 1895), Kiriwana und Woodlark Inseln (Thomas 1896), Salomon-Inseln (Shortland- 
Inseln, Alu, Thomas 1887, Guadalkanar, Aola, Thomas 1888, Neu-Georgien, Dobson), Neu-Pommern (Matupi, B. M.), Neu-Lauenburg (Dobson 1878).

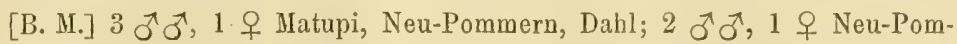
mern, Finsch.

\section{Genus. Leiponyx Jentink.}

1881. Leiponyx, Jentink, Not. Leyd. Mus. III, p. 59-61.

Grosse Flederhunde mit wohl entwickeltem Schwanze. Der Zeigefinger ist ohne Kralle. Der Rand des Ohres bildet wie bei den übrigen Megachiroptera einen ovalen Ring. Die Flughaut setzt sich an die erste Zehe an und ist an die Seiten des Rückens angeheftet. Der Metacarpus des Mittelfingers ist kleiner als der Zeigefinger. Die Ränder der Nasenlöcher sind namentlich am Innenrande etwas aufgetrieben. Die Schnauze ist derjenigen von Pteropus nach Jentink ähnlich. Die Oberlippe zeigt eine senkrechte Grube. Gebiss $\frac{2.1 .2 .2}{2.1 .3 .3}$. Die Schneidezähne stehen paarweise und sind auch von den Eckzähnen durch eine Lücke getrennt. Zwischen dem Eckzahn und dem nächsten Praemolar ist eine kleine Lücke. Die Molaren sind sehr klein. Auf dem Gaumen befinden sich 4 ungetheilte Falten und dahinter jederseits drei ovale Wülste, worauf einige gezähnelte Querfalten folgen.

Nach Büttikofer ist der Flug dieser Thiere ruhig, etwas eulenartig und schwankend. Sie zogen Ende October in zahlreichen Exemplaren hoch über die Mission hin nach Süden.

Typus: Leiponyx büttikoferi Jentink.

Hab. Liberia, West-Afrika.

Nur eine Art: L. büttikoferi Jentink. - 1881. Leiponyx büttikoferi Jentink vom St. Paul's Flusse in Liberia, Not. Leyd. Mus. IlI, p. 60-61. - 1888. Jentink und Büttikofer, 1. c. X, p. 53. - 1897. Trouessart, 1. c. p. 89.

Hab. St. Paul's Fluss, Liberia.

\section{Genus. Cephalotes E. Geoffroy St. Hilaire.}

1810. Cephalotes E. Geoffroy St. Hilairê, Ann. Mus. XV, p. 101-106.

1828. Hypoderma Geoffroy St. Hilaire, Cours de l'Hist. Nat. des Mamm. I, 13, Lecon, 27. Juni, p. 29. - 1869. Cephalotes Fitzinger, Sitzb. Ak. Wien LX, 1. Abth. p. 53-57. - 1878. Dobson, Cat. Chiropt. p. 91-92. - 1887. Jentink, Mus. d'Hist. Nat. Pays-Bas, IX, Cat. Osteol. p. 267-268. - 1888. Jentink, I. c. XII, Cat. Syst. p. 156-158. - 1897. Trouessart, Cat. Mamm. Nov. 
Ed. I, p. 87-88. - 1898. Dobsonia* T. S. Palmer, Proc. Biol. Soc. Washington, XII, p. 114.

Grosse Fledermäuse mit wohl entwickeltem Schwanze, der zum Theil mit der Schenkelflughaut verwachsen ist. Der Zeigefinger ist krallenlos. Der Rand des schmalen, langen Ohres bildet einen ovalen Ring. Die Flughaut setzt sich an die erste und zweite Zehe an und ist auf der Mittellinie des Rückens angeheftet, so dass der Rücken vollständig von ihr verdeckt wird. Den Metacarpus des Mittelingers ist ungefähr so lang wie der Zeigefinger. Die Ränder der Nasenlöcher sind auf dem Vorderrande wulstig aufgetrieben, aber nicht röhrenförmig verlängert. Die Schnauze ist kurz und dick, vorn abgeplattet. Keine mittlere Furche auf der Lippe zwischen der. Nase und dem Lippenrande. Ober- und Unterlippe am Innenrande mit einer Reihe hervorragender spitzer Schleimhautfortsätze. Gebiss: $\frac{1.1 .2 .2}{1.1 .3 .3}$. Die beiden oberen Schneidezähne sind von den Eckzähnen und bei alten Thieren auch von einander durch eine Lücke getrennt. Die Praemaxillaria sind nicht verwachsen. Im Milchgebiss sind oben und unten 4 Incisiven vorhanden; bei ganz alten Thieren rücken die unteren Caninen mit ihrer Basis soweit zusammen, dass die kleinen Incisiven sämmtlich ausfallen.

Auch der letzte kleine obere Molar geht zuweilen verloren. Im Oberkiefer ist eine kleine Lücke zwischen den Praemolaren und dem Caninus. Die Molaren haben bei jüngeren Thieren, wie diejenigen von Acerodon, stark vorspringende Höcker und Wülste. Der knöcherne Gaumen reicht weit über die Molarenreihe hinaus. - Der Schädel ist sehr kurzschnauzig, der Postorbitalfortsatz des Frontale ist stark entwickelt. - Auf dem Gaumen folgen auf vier ganzrandige, ungetheilte Falten 7-8 getheilte, am Rande gesägte Falten. - Saugwarzen auf den Brustseiten etwas unter der Achselgrube. Ruthe des Männchens ohne Penisknochen. Schwanzflughaut nicht breit; sie wird durch einen Bindegewebsstrang gestützt, der vom Schwanze zum Fussgelenk ungefähr parallel dem Unterrande der Flughaut sich erstreckt. Auf der Ellenbogen-Flughaut, dem Plagiopatagium, wird der vom Ellenbogen zur Spitze des fünften Fingers verlaufende Strang von mindestens 20 ungefähr parallelen Nebensträngen fast rechtwinklig durchschnitten.

Schon. Temminck hat darauf aufmerksam gemacht, dass die Cephalotes in den Maasverhältnissen sehr variiren; er vermuthet, dass lokale Abarten vorhanden sind und weist darauf hin, dass die Exemplare von Timor und Amboina einen längeren Kopf haben als die von Banda und Samao.

Das Berliner Museum hat z. B. von Batjan zwei Weibchen mit stark geschwollenen Zitzen; der Unterarm des einen ist $106 \mathrm{~mm}$, derjenige des anderen $125 \mathrm{~mm}$ lang; das kleinere Weibchen hat eine Fusslänge von $24 \mathrm{~mm}$ und eine Kopflänge von $49 \mathrm{~mm}$, das grössere von $27 \mathrm{~mm}$ resp. $57 \mathrm{~mm}$. Ferner stehen in unserer Sammlung u. a. zwei Stück von Key; der Fuss des $\sigma^{7}$ ist $25 \mathrm{~mm}$, derjenige des ㅇ $20 \mathrm{~mm}$ lang bei einer Unterarmlänge von $121^{-}$resp. $113 \mathrm{~mm}$. Ein ganz junges 우 im Zahnwechsel von Ceramlaut hat einen Unterarm von $96 \mathrm{~mm}$ Länge; sein Kopf ist $53 \mathrm{~mm}$ und sein Fuss $28 \mathrm{~mm}$ lang. Ein anderes nicht viel älteres $\widetilde{\sigma}$ von Neu-Lauenburg ist ebenso gross, hat aber nur eine Kopflänge von $44 \mathrm{~mm}$ und eine Fusslänge von $24 \mathrm{~mm}$.

Ich finde nicht, dass derartige Verschiedenheiten in den Maassen auf geographische Abarten hinweisen und stelle die fünf bisher beschriebenen Cephalotes-Species in einer einzigen Art zusammen.

* Aus den oben (p. 81) vorgetragenen Gründen bezieht sich der Name Cephalotes nicht auf $\nabla$. cephalotes, sondern auf Cephalotes peronii. 'Die Aufstellung des neuen Gattungsnamens Dobsonia halte ich für unnöthig. 


\section{mon = Inseln. \\ Hab. Nord-Celebes, Molukken, Neu-Guinea, Bismarck-Archipel, Salo-}

Wahrscheinlich nur eine Art: C. palliatus (E. Geoffroy St. Hilaire). - 1810. Pteropus paliatus E. Geoffroy St. Hilaire von unbekannter Herkunft, Ann. Mus. XV, p. 99-101. - 1810. Cephalotes Peronii E. Geoffroy St. Hilaire von Timor, Ann. Mus. XV, p. 104-106, Taf. 7 (Thier, Schädel von oben und von der Seite, Gebiss von vorn). - 1828. Hypoderma Peronii Isidor Geoffroy St. Hilaire, Dict. Class. d'Hist. Nat. XIV, p. 706. - 1830. Hypoderma moluccense Quoy und Gaimard von Amboina, Voyage de L'Astrolabe I, p. 86-88, Taf. 11, Fig. 1 (Thier), 2 (Schädel), 3 und 4 (Incisivi und Canini im Ober- und Unterkiefer), 5-7 (Aeussere Geschlechtsorgane), 8 (Magen). - 1830. Harpyia Peronii, Wagler, Natürl. Syst. Amphib. p. 10. - 1837. Cephalotes Peronii Temminck, Monogr. Mammal. II, p. 106-110, Taf. 35, Fig. 7 (Kopf), Taf. 36, Fig. 24 (Schädel von der Seite), 25 (Schädel von .oben), 26 (Schädel von unten), 27 (Unterkiefer von oben), 28 (Praemaxillaren, Incisiven, Caninen), 29 (Praemaxillaren). - 1843. Xantharpyia amplexicaudata Gray, Voyage Sulphur, Mamm. p. 30. - 1869. Cephalotes Peronii Fitzinger, Sitzb. Ak. Wiss. Wien LX, 1. Abth. p. 53-57. - 1878. Dobson 1. c. p. 91-92. - 1878 (?) Cephalotes minor Dobson von Amberbaki, Neu-Guinea, Proc. Zool. Soc. London, p. 875. - 1880. Dobson, Rep. British Assoc. 1880, p. 6. - 1881. Cephalotes Peronii Peters und Doria, Ann. Mus. Civ. Genova p. 691. - 1887. Jentink, Cat. Ost. p. 267-268. - 1887. Thomas, Proc. Zool. Soc. London p. 323. - 1888. Thomas, I. c. p. 476. - 1888. Jentink, Cat. Syst. p. 156-158. - 1895. Thomas, Nov. Zool. II, p. 163. - 1896. Thomas, 1. c. III, p. 526. - 1896. Heude, Mém. Hist. Nat. Empire Chinois III, p. 176, Anmerkung (1), Taf. V, Fig. 2 (Gebiss eines Exemplars von Flores). - 1896. Cephalotes viridis Heude von den KeyInseln, l. c. p. 176, Anm. (1), Taf. V, Fig. 1 (Gebiss). - 1897. Cephalotes Peronii Trouessart, l. c. p. 87. - 1897. Thomas, Ann. Mus. Genova (XIX) (38) p. 608. - 1898. Hartert, Nov. Zool. V, p. 456. - 1898. Dobsonia peronii und D. minor Palmer, Proc. Biol. Soc. Washington, XII, p. 114.

Hab. Nord-Celebes, Molukken, Neu-Guinea, Bismarck-Archipel, SalomonInseln, im besonderen Flores (IIeude), Timor, Wetter, Samao (Jentink), Alor (Hartert), Banda, Amboina (Jentink), Ceram (Dobson), Ceramlaut (Berl. Mus.), Buru, Nord-Celebes, Batjan, Ternate, Rau, Morotai, Misol, Waigeu, Mafor, Aru-Inseln (Jentink), Key-Inseln (Peters und Doria), Neu-Guinea (Andai, Jentink; Amberbaki, Dobson; Bongu und Salawatti, Heller; Ighibirei am Kamp Welch Fluss, Thomas 1897), Neu-Pommern, Neu-Lauenburg (Jentink), Kiriwana-Inseln (Thomas 1896), D'Entrecasteaux-Inseln (Fergusson-Insel, Thomas 1895), Salomon-Inseln (Ugi, San Christoval, Thomas 1887; Neu-Georgien, Thomas 1888, Shortland-Insel, Thomas 1887).

[B. M.] ot Timor, Reinwardt; $q$ Manado, A. B. Meyer; 2 우 Batjan, Bernstein; $q$ juv. Ceramlaut, Beccari; $ᄋ$ Astrolabe Bay, Neu-Guinea-Gesell-

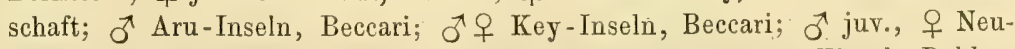

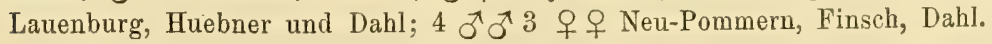




\section{Genus. Notopteris Gray.}

1859. Notopteris Gray, Proc. Zool. Soc. London, p. 36-38.

1878. Dobson, Cat. Chiropt. p. 92-94. - 1897. Trouessart, Catal. Mamm. Nov. Ed. I, p. 90.

Mittelgrosse Flederhunde. Der Schwanz ist fast so lang wie der Unterarm und an seiner Wurzel mit der schmalen Schwanzflughaut verwachsen. Der Zeigefinger ist krallenlos. Der Rand des Ohres bildet einen ovalen Ring, wie bei allen Megachiroptera. Die Flughaut setzt sich an den Fussrücken hinter der zweiten Zehe an und entspringt wie bei Cephalotes von der Rückenmitte, so dass der Rücken vollständig von der nackten Flughaut bedeckt ist. Der Metacarpus des Mittelfingers ist ungefähr so gross wie der Zeigefinger. Die Ränder der halbmondförmigen Nasenlöcher sind nicht besonders aufgetrieben. Die Schnauze ist sehr lang und schmal. Ueber die Mitte der Nasenscheidewand verläuft bis zum Lippenrande eine Furche. Gebiss: $\frac{2.1 .2 .2}{1.1 .2 .3}$.

Die inneren oberen Schneidezähne, welche seitlich vor den äusseren stehen, sind so klein, dass sie kaum den Alveolarrand überragen. Die Praemaxillaria sind vorn durch eine Naht verbunden. Die äusseren oberen Schneidezähne sind von den Eckzähnen durch eine Lücke getrennt. Letztere sind sehr gross und haben eine Längsfurche auf der Vorderseite. Die beiden Molaren-Reihen sind fast parallel. Der erste Praemolar schliesst sich eng an den Eckzahn an, ist höher als die übrigen Molaren und hat eine scharfkantige, meisselförmige Krone; er ist vom zweiten Praemolar durch eine Lücke getrennt. Die letzten drei Zähne im Oberkiefer sind einander ähnlich, der zweite Holar ist etwas kleiner als die übrigen. Ihre Gestalt ist ellipsenförmig. Im Unterkiefer sind die beiden Schneidezähne sehr klein und stehen dicht neben den nach hinten gerichteten Eckzähnen. Die beiden Praemolaren haben eine meisselförmige Krone und sind von einander durch eine Lücke getrennt; der erste Praemolar steht dicht neben dem Eckzahne. Der knöcherne Gaumen reicht weit über die Molarenreihe hinaus. Auf dem Gaumen sehe ich 8 ungetheilte Querfalten, von denen die beiden letzten gezähnelt sind. Vor den Eckzähnen stehen noch zwei kurze Querfalten. Die Zunge läuft nach vorn sehr spitz zu; auf ihrem vorderen Ende befindet sich ein mittleres vertieftes Längsfeld, welches mit grösseren, in schiefẹn Reihen stehenden Hornpapillen besetzt ist. Der vom Ellenbogen zur Spitze des fünften Fingers auf dem Plagiopatagium verlaufende Strang wird von ungefähr 12 Nebensträngen fast senkrecht durchschnitten. Die Saugwarzen liegen dicht unter der Achselhöhle an den Körperseiten.

linen (Ponape).

Hab. Fidschi-Inseln (Witi Lewu, Ovalau), Neu-Hebriden (Aneiteum), Karo-

Nur eine Art: N. macdonaldii Gray. - 1859. N. Macdonaldii J. E. Gray von

Witi Lewu, Proc. Zool. Soc. London, p. 38, Taf. LXVII (Thier, Schädel von der Seite, Incisivi, Canini und erster Praemolar oben und unten). - 1878. Dobson 1. c. p. 93 -94. - 1880. Thomas, Proc. Zool. Soc. p. 11. - 1887. Jentink, Cat. Ost. p. 268. - 1888. Jentink, Cat. Syst. p. 158. - 1897. Trouessart l. c. p. 90 .

Hab. Fidschi-Inseln (Witi Lewu, Gray; Ovalau, Thomas), Neu-Hebriden (Aneiteum, Dobson), Karolinen (Ponape, Jentink).

[B. M.] ฮ 2 우우 Witi Lewu, Godeffroy. 


\section{Genus. Eonycteris Dobson.}

1873. Eonycteris Dobson, Proc. As. Soc. Bengalen, XLII, p. 204.

1871. Macroglossus partim Dobson, Proc. Ac. Soc. Bengalen XXXIX, p. 261. - 1878. Eonycteris, Dobson, Cat. Chiropt. p. 94-95. - 1881. Eonycteris, Anderson, Cat. Mamm. Ind. Mus. p. 106-107. - 1888. Eonycteris partim, Jentink, Mus. d'Hist. Nat. Pays-Bas IX, Cat. Syst. p. 158. - 1891. Eonycteris, Blanford, Fauna Brit. India, Mamm. p. 266. - 1897. Eonycteris Trouessart, Cat. Mamm. p. 266.

Mittelgrosse Flederhunde. Der Schwanz ist kurz, aber wohl entwickelt und im Basaltheile mit der Schwanzflughaut verwachsen. Diese ist in der Mitte ziemlich schmal, reicht aber in einer geraden Linie bis zu den Fusswurzeln. Der Zeigefinger ist krallenlos. Der Rand des Ohres bildet wie bei den übrigen Flughunden einen ovalen Ring. Die Flughaut setzt sich an die Rückenseiten und an die erste Zehe an oder an den Fussrücken kurz hinter der Stelle, wo die erste und zweite Zehe sich trennen. Der Metacarpus des Mittelfingers ist ungefähr so lang wie der Zeigefinger. Die Ränder der Nasenlöch er sind nicht aufgetrieben; die Nasenlöcher sind oval mit einer langen, schmalen hinteren Ausbuchtung. Die Schnauze ist sehr lang und schmal. Ueber die Mitte der Nasenscheidewand bis zum Lippenrande, ebenso über die Mitte des vorderen Unterkieferrandes verläuft je eine vertikale Furche. Gebiss: $\frac{2.1 .3 .2}{2.1 .3 .3}$. Im Unterkiefer bilden die sehr kleinen Schneidezähne ein Trapez; die grossen Eckzähne haben vorn und hinten je eine Furche; der erste Praemolar ist so gross wie die äusseren Schneidezähne, der zweite ist viel höher als der dritte, so lang wie der Eckzahn und vom dritten Praemolar durch eine kleine Lücke getrennt; der letzte Molar ist kaum halb so gross wie der erste Molar. Im Unterkiefer ist ebenfalls der erste Praemolar sehr klein, der zweite ist vom ersten etwas weiter entfernt als vom dritten. Die echten Molaren sind in beiden Kiefern sehr schwach und viel länger als breit. Die Praemaxillaren sind nicht verwachsen. Der knöcherne Gaumen reicht weit über die Molarenreihe hinaus. Auf dem Gaumen stehen hinter vier ungetheilten Querfalten drei getheilte, von denen die letzte Spuren eines Sägerandes zeigt. Dahinter befindet sich eine ungetheilte, gezähnelte Querfalte. Die Zunge ist sehr lang, läuft nach vorn spitz zu und ist im vorderen Ende oben mit langen, spitzen, rückwärts gerichteten Papillen dicht bedeckt; es folgt dann ein nach hinten spitzwinklig verlaufendes Feld von breiten, hinten dreispitzigen Papillen und an den Seiten der Zungenwurzel wieder ein solches mit spitzen, einwärts gerichteten, langen Papillen. Auf dem Plagiopatagium wird der vom Ellenbogen gegen die Spitze des fünften Fingers ziehende Strang von ungefähr zwölf Nebensträngen durchschnitten. - Die Saugwarzen liegen dicht unter der Achselhöhle an den Körperseiten. Auf beiden Seiten der Analöffnung befindet sich je ein drüsenartiger Wulst bei beiden Geschlechtern.

Zur Fortpflanzungszeit tritt bei den Männchen der Hodensack dicht neben dem rechten Drüsenwulst hervor. Eonycteris hält sich bei Tage in Höhlen auf.

\section{Hab. Hinterindien, Grosse Sunda-Inseln.}

Nur eine Art: E. spelaea (Dobson). - 1871. Macroglossus spelaeus (Dobson) aus den Farm-Caves nahe bei Moulmein, Tenasserim, Proc. As. Soc. Bengalen, p. 106. - 1871. Dobson, Journ. As. Soc. Bengalen, XL, 2. Theil, p. 261-262, Taf. X, Fig. 3 (Unteres Körperende), 4. [Schädel von der. Seite, Oberkiefer (b), 
Unterkiefer (a)]. - 1873. Eonycteris spelaea, Dobson, Journ. As. Soc. Bengalen, XLII, 2. Theil, p. 204, Taf. XIV, Fig. 10 (Ohr). - 1878. Dobson, l. c. p. 94-95. - 1878. Dobson, Proc. Zool. Soc. London p. 877. - 1881. Anderson, Cat. Mamm. Ind. Mus. I, p. 106-107. - 1888. Jentink, Cat. Syst. p. 158. - 1888. Fea, Soc. Geogr. Ital. Juli-Heft. - 1890. Jentink, Weber's Zool. Erg. I, 1, p. 96 und 126-127. - 1891. Blanford. Fauna British India, Mamm. p. 266-267. 1892. Thomas, An. Mus. Civ. Genova, 2. ser. XXX, p. 922. - 1893. Everett, Proc. Zool. Soc. p. 494. - 1893. Hose, Acc. Mamm. Borneo, p. 39. - 1897. Trouessart, 1. c. p. 89.

Hab. Nord-Tenasserim (Farm Caves bei Moulmein Dobson, 1871), UnterBirma (Carin Berge am Saluen, nordöstliches Unter-Birma Thomas, 1892), Malakka (Perak, B. M.), Sumatra (Sinkarah, Jentink 1890), Java (Dobson, 1878), Borneo (Sarawak, Everett; Darvel Bay, Nordost-Borneo, B. M.).

[B. M.] ㅇ Moulmein, Theobald, vom Calcutta-Museum; 우 pull. Java, durch Tausch von Temminck erhalten, ơ Perak, Malakka, Hartert; $ᄋ$ mit Embryo, Lah Datu, Darvel Bay, Nordost-Borneo, Pagel.

\section{Genus. Callinycteris Jentink.}

1889. Callinycteris Jentink, Not. Leyd. Mus. XI, p. 209-212.

1888. Eonycteris partim Jentink, Cat. Syst. p. 158. - 1897. Trouessart, Cat. Mamm. Nov. Ed, I, p. 90.

Nach der Original-Beschreibung unterscheidet sich Callinycteris von Eonycteris dadurch, dass die Flughaut an die zweite, nicht aber an die erste Zehe angeheftet ist, dass im Unterkiefer nur 5 Molaren sitzen und dass die Analöffnung nicht etwas vor den Drüsenwülsten, sondern in der Mitte zwischen ihnen sich befindet.

Auf zwei von diesen Merkmalen möchte ich nicht allzu grossen Werth legen. Bei einem Exemplare von Eonycteris reicht die Flughaut nicht bis zur Wurzel der ersten Zehe, sondern hört kurz vor der Stelle auf, wo die erste und zweite Zehe sich trennen. Diese Anordnung würde also eine Mittelform zwischen Eonycteris und Callinycteris darstellen. Ferner zeigen zwei von unseren Eonycteris-Stücken, dass während der Fortpflanzungszeit die neben der Analöffnung vorhandenen, merkwürdigen Drüsenwülste erheblich anschwellen und dass dann die Afteröffnung mitten zwischen den Drüsenkörpern sich befindet.

Auf den. Abbildungen, welche Herr Jentink giebt, scheinen sich aber einige Unterschiede zwischen der Celebes-Form und derjenigen von den Sunda-Inseln und Hinter-Indien zu ergeben: Hinter der dritten getheilten Gaumenfalte ist bei Eonycteris noch eine gerade, ungetheilte und gezähnelte Querfalte zu finden, welche auf der Abbildung für Callinycteris nicht angegeben wird. Ferner unterscheidet sich die Zunge von Callinycteris, wie die Abbildung zeigt, dadurch von der Zunge unserer Eonycteris-Exemplare, dass die Zụngenspitze durchaus keine langen, spitzen, nach hinten gerichteten Papillen trägt. Endlich ist bei Eonycteris der vierte Oberkieferzahn hinter dem Eckzahn der längste Zahn der Reihe, 
während nach der Abbildung bei Callinycteris der dritte Oberkieferzahn hinter dem Eckzahn die grösste Länge hat.

Die Gestalt der Nasenlöcher und Lippen ist bei Eonycteris genau so, wie es Jentink für Callinycteris abbildet.

\section{Hab. Nord-Celebes (Gorontalo).}

Nur eine Art: C. rosenbergi Jentink. - 1888. Eonycteris spelaea Variété insulaire Jentink, Cat. Syst. p. 158. - 1889. Callinycteris rosenbergi Jentink von Gorontalo auf Nord-Celebes, Not. Leyd. Mus. XI, p. 210-212, Taf. 9, Fig. 1 (Oberlippe mit den Nasenlöchern, Unterlippe), 2 (Gaumenfalten und Oberkieferbezahnung), 3 (Zunge), 4 (Penis, Analöffnung und Drüsenwülste). - 1897. Trouessart, 1. c. p. 90.

\section{1\%. Gattung: Nesonyeteris Thomas.}

1887. Nesonycteris Thomas, Ann. Mag. Nat. Hist. (5) XIX, p. 147. I. c. p. 90 .

1887. Thomas, Proc. Zool. Soc. London, p. 323-326. - 1897. Trouessart,

Kleine Flederhunde ohne deutlichen Schwanz: Die Schwanzflughant ist in der Analgegend von der Behaarung verdeckt. Zeigefinger ohne Kralle. Der Rand des Ohres bildet einen ovalen Ring. Die Flughaut setzt sich an die Körperseiten und an die Wurzel der dritten Zehe an. Der Metacarpus des Mittelfingers ist kürzer als der Zeigefinger. Die Ränder der Nasenlöcher sind aufgeworfen. Die Schnauze ist sehr lang und schmal; über die Mitte der Nasenscheidewand bis zum Lippenrande verläuft eine senkrechte Furche. Gebiss: $\frac{2.1 .3 .2}{1.1 .3 .3}$. Im Oberkiefer stehen die Schneidezähne im Bogen; die Eckzähne sind lang und kräftig und haben je eine tiefe Längsgrube an der Vorder-, Aussen- und Hinterseite. Der erste Praemolar ist sehr klein und an den Eckzahn angedrückt. Die Molaren sind sehr schmal; der letzte ist ungefähr $2 / 3$ so gross wie der erste. - Im Unterkiefer sind die beiden, dicht an den Eckzahn gedrängten Schneidezähne durch eine weite Lücke von einander getrennt. Der erste Praemolar berührt den Eckzahn, zwischen ihm und dem zweiten Praemolar ist ein breites Diastema. Die Praemaxillaria sind vorn getrennt. Der Unterkiefer hat wie bei Macroglossus und Melonycteris eine lange Symphyse, der Unterkiefer ist vor dem zweiten Praemolaren am schmalsten. - Auf dem Gaumen sind die hinteren Querfalten in der Mitte getheilt.

\section{Hab. Salomon-Inseln.}

Nur eine Art: N. woodfordi Thomas. - 1887. Nesonycteris woodfordi Thomas von den Shortland- und der Fauro-Insel, westliche Salomon-Inseln, Ann. Mag. Nat. Hist. (5), XIX, p. 147. - 1887. Thomas, Proc. Zool. Soc. London, 
p. 324-326, Taf. XXVI (Thier), p. 325, Fig. 2 (Schädel von oben), Fig. 3 (Schädel von der Seite). - 1888. Thomas, Proc. Zool. Soc. London, p. 476. 1897. Trouessart, l. c. p. 90.

Hab. Westliche Salomon-Inseln.

\section{Gattung: Melonycteris Dobson.}

1877. Melonycteris, Dobson, Proc. Zool. Soc. p. 119-121. - 1877. Cheiropteruges Ramsay, Proc. Linn. Soc. New South Wales II, p. 17-19. - 1888. Jentink, Cat. Syst. p. 159-160. - 1897. Trouessart 1. c. p. 90.

Kleine Flughunde ohne deutlichen Schwanz. Die Schwanzflughaut ist in der Analgegend von der Behaarung verdeckt. Zeigefinger mit Kralle. Der Rand des Ohres bildet einen ovalen Ring. Die Flughaut setzt sich an die Körperseiten und den Fussrücken hinter der dritten Zehe an. Der Metacarpus des Mittelfingers ist ungefähr so lang wie der Zeigefinger. Die Innenränder der Nasenlöcher sind etwas aufgeworfen. Die Schnauze ist sehr lang und schmal; über die Mitte der Nasenscheidewand verläuft bis zum Lippenrande eine senkrechte Furche. MuffeI am Lippenrande breiter als a u der Mitte der Oberlippe. Gebiss: $\frac{2.1 .3 .2}{2.1 .3 .3}$ sehr ähnlich dem Gebisse von Eonycteris; aber im Oberkiefer sind die Eckzähne an der Aussenseite mit einer Längsrippe versehen und haben ausserdem vorn und hinten je eine Längsfurche; der erste Praemolar ist viel kleiner als der äussere Schneidezahn; der zweite Praemolar ist nicht so lang wie der Eckzahn und seine Entfernung vom dritten Praemolaren ist viel grösser als seine eigene Länge. Der letzte Molar ist fast so gross wie der erste Molar, welcher dem letzten Praemolaren an Länge gleicht. Die Praemaxillaren sind vorn getrennt. - Im Unterkiefer ist Melonycteris sehr ähnlich Nesonycteris dadurch, dass die Symphyse sehr lang und der Unterkiefer vor dem zweiten Praemolaren am schmalsten ist. Der erste Praemolar ist sehr klein, der zweite Praemolar ist vom ersten fast doppelt so weit entfernt wie rom dritten. - Die Molaren in beiden Kiefern sind sehr schwach und klein. Das Palatinum reicht weit über die Zahnreihe hinaus. Auf dem Gaumen stehen hinter fünf ungetheilten zwei getheilte Querleisten und vor den Eckzähnen ist eine winklig gebogene Falte sichtbar, an deren Rande die Schneidezähne stehen. - Die Zunge ist sehr lang; an der Spitze von kleinen, hornigen, nach hinten gerichteten und am Ende zugespitzten Papillen bedeckt, auf der Mitte der vorderen Zungenhälfte befinden sich im länglichen Feld grössere, am Hinterrande mit drei Spitzen versehene Papillen. - Auf dem Plagiopatagium wird der vom Ellenbogen gegen die Spitze des fünften Fingers verlaufende Strang von ca. acht stärkeren Nebensträngen ziemlich senkrecht durchschnitten. Zahlreiche zum unteren Rande der Flughaut parallele Fascien laufen ungefähr mit dem Hauptstrange in gleicher Richtung. Die Saugwarzen liegen etwas unterhalb der Achselhöhle auf den Körperseiten. Die Ruthe des Männchens hat keinen Penisknochen.

\section{Hab. Neu-Guinea und Bismarck-Archipel.}


Nur eine Art: M. melanops Dobson. - 1877, Juni. Melonycteris melanops Dobson von einer Reise nach Neu-Lauenburg und den angrenzenden Theilen von Neu-Pommern und Neu-Mecklenburg, Proc. Zool. Soc. London, p. 119-121, Taf. XVII (Thier), p. 119, Fig. 4 (Schnauze mit den Nasenlöchern), Fig. 5 (Ohr); p. 120, Fig. 6 (Unterkieferhälfte von oben), Fig. 7 (Oberkieferhälfte von unten). 1877, Juli. Pteropus (Cheiropteruges) alboscapulatus Ramsay von Neu-Lauenburg, Proc. Linn. Soc. New South Wales, p. 17-19. - 1878. M. melanops Dobson, l. c. p. 97-98. - 1888. Jentink, Cat. Syst. p. 159-160. — 1897. Trouessart l. c. p. 90.

\section{Hab. Neu-Lauenburg, Neu-Pommern, Neu-Guinea.}

[B. M.] 1 Ј 5 우 우, 5 Embryonen, Ralum, Neu-Pommern, Dahl; 오, 우 juv.

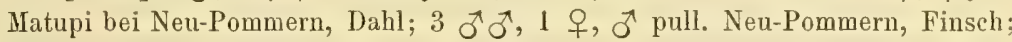
우 Neu-Lauenburg, Finsch, $\widehat{\jmath}$ juv. Mac Lure Bay, Südwest-Neu-Guinea, S. M. S. Gazelle.

\section{Gattung: Macroglossus F. Cuvier.}

1825. Macroglossus F. Cuvier, Dents des Mammif. p. 40-41, 248. - 1827. Macroglossus Lesson, Manuel de Mammal. p. 115. - 1840. Kiodotus (fide Palmer, Proc. Biol. Soc. Washington, XII, p. 111) Blyth Cuvier's Animal Kingdon p. 60, Anmerkung. - 1869. Macroglossus Fitzinger, Sitzb. Akad. Wien, LX, 1. Abth. November p. 21-24. - 1878. Dobson, Cat. Chiropt. p. 95-97. - 1887. Jentink, Cat. Syst. p. 268-269. - 1887. Thomas, Proc. Zool. Soc. London, p. 324. 1891. Carponycteris Lydekker in Flower und Lydekker, Introduction to the Study of Mammals p. 654. - 1891. Blanford, Fauna Brit. India, Namm. p. 265-266. 1897. Trouessart, Cat. Mamm. Nov. Ed. I, p. 90.

Sehr kleine Flughunde ohne deutlichen Schwanz. Die Schwanzflughaut ist in der Analgegend von der Behaarung verdeckt. Zeigefinger mit Kralle. Der Rand des Ohres bildet einen ovalen Ring. Die Flughaut setzt sich an die Körperseiten und an den Fussrücken hinter der vierten Zehe an. Der Metacarpus des Mittelfingers ist mindestens ebenso lang wie der Zeigefinger. Die Innenränder der Nasenlöcher sind nicht aufgeworfen. Die Schnauze ist sehr lang und schmal; über die Mitte der Nasenscheidewand verläuft eine Längsfurche, welche bei den meisten hierher gehörigen Formen bis zum Lippenrande reicht. M u ffel am Lippenrande ungefähr so breit wie auf der Mitte der Oberlippe, nicht am Lippenrande verbreitert. Gebiss: $\frac{2.1 .3 .2}{2.1 .3 .3}$; die Zähne mit Ausnahme der Eckzähne sind sehr klein und schwach. Im Oberkiefer haben die Eckzähne vorn eine Furche; die Schneidezähne stehen im Trapez; der erste Praemolar ist ungefähr so gross wie der zweite Praemolar und nicht a uffallend klein; der letztere 
überragt nur wenig den dritten Praemolar. Der letzte Molar ist sehr klein. Entweder stehen alle Oberkieferzähne hinter dem Eckzahn in gleichen Abständen oder der erste Praemolar steht vom zweiten Praemolar etwas weiter entfernt als dieser vom dritten. Im Unterkiefer sind die Eckzähne nah hinten gerichtet, die Backenzähne ausser dem letzten Molar ungefähr gleich gross und in ziemlich gleichem Abstande von einander oder aber der zweite Praemolar ist vom ersten und dritten Praemolar durch eine weite Lücke getrennt. Die Symphyse ist nicht lang, der Unterkiefer verjüngt sich nach vorn gleichmässig. Das Palatinum reicht weit über die Zahnreihe hinaus. Auf dem Gaumen stehen sieben ungetheilte Querfalten. - Die Zunge ist sehr lang, die Papillen sind ähnlich wie bei Melonycteris. Bei einigen Exemplaren sind auf der hinteren Zungenhälfte ca. 12 grössere flache Papillen deutlich. Auf dem Plagiopatagium wird der vom Ellenbogen gegen die Spitze des fünften Fingers verlaufende Strang von 8-12 Nebensträngen ziemlich senkrecht durchschnitten; $6-8$ dem unteren Flughautrande parallele Fascien befinden sich unterhalb des proximalen Theiles des Hauptstranges. - Die Saugwarzen liegen auf den Körperseiten etwas unter und vor der Achselhöhle. Ein Penisknochen scheint den Männchen zu fehlen.

Die Macroglossus-Flederhunde hängen bei Tage in den Zweigen, kommen nach Horsfield auch in die Hausdächer und sollen Früchte fressen. Ich habe im Enddarm eines Stïckes von Neu-Pommern Reste einer feigenartigen Frucht gefunden. Nach Temminck stossen sie durchdringende, gellende Töne aus. Lydekker hat vorgeschlagen, den Namen Macroglossus zu verwerfen, weil von Scopoli viel früher eine Gattung Macroglossum beschrieben worden ist. Ich schliesse mich Trouessart an, der etymologisch gleich abgeleitete, aber durch verschiedene Endsilben als verschieden gekennzeichnete Namen annimmt, wie es übrigens auch die Deutsche Zoologische Gesellschaft vorgeschlagen hat.

Bis jetzt sind drei Arten von Macroglossus aufgestellt worden. M. minimus Geoffr., M. australis Ptrs. und $M$. crassus Thos. Schon Temminck hat (Monogr. Mamm. II p. 97) behauptet, dass die Macroglossus von Sumatra, Java, Timor, Amboina und Celebes gewisse Unterschiede zeigen in der Länge der Schnauze, hat aber keine Namen für die von ihm vermutheten Lokalformen aufgestellt. Peters beschrieb einen Macroglossus von Rockhampton als $M$. australis, Thomas einen solchen von der Fergusson-Insel als Carponycteris crassa. Beide stehen einander sehr nahe und sind durch eine Anzahl guter Merkmale von $M$. minimus unterschieden; diese Unterschiede sind so wesentlich, dass ich zwei Untergattungen von Macroglossus annehmen muss: 1. Macroglossus mit sehr kleinen Schneidezähnen, einem Diastema zwischen dem ersten und zweiten Praemolar in beiden Kieferu und wohl ausgebildeter Schwạnzflughaut; 2. Syconycteris (von oũxov $=$ Feige und vúzrepıs $=$ Fledermaus) mit grossen Schneidezähnen, geschlossener Molarenreihe und verkümmerter Schwanzflughaut.

Hab. Von Darjiling über Hinterindien, die Sunda-Inseln und Philippinen bis zum Bismarck-Archipel, den Salomon-Inseln und Nord-Australien. 


\section{Uebersicht der Untergattungen.}

Schneidezähne kürzer als der erste Molar; die mittleren stehen mindestens ebenso weit auseinander wie die äusseren;

Schwanzflughaut am Knie breiter als $4 \mathrm{~mm}$. . . . . Macroglossus Geoffr. p. 95

Schneidezähne viel länger als der erste Molar, die mittleren stehen näher zusammen als die äusseren; Schwanzflughaut

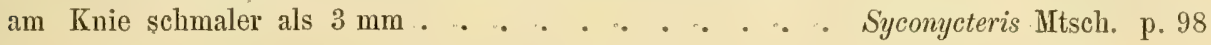

\section{Uebersicht der geographischen Verbreitung.}

\begin{tabular}{c|c|c}
\hline & Macroglossus & Syconyeteris \\
\hline Hinterindien & als minimus & - \\
Sumatra & als minimus & - \\
Java & minimus & - \\
Borneo & lagochilus (?) & - \\
Celebes & lagochilus (?) & - \\
Philippinen & als australis & - \\
Amboina-Gruppe & lagochilus & - \\
Timor, Flores & als minimus & papuanus \\
Nord- und Süd-Neu-Guinea & nanus & papuanus (?) \\
Aru-Inseln & nanus & finschi \\
Bismarck-Archipel & nanus & crassus \\
Südost-Neu-Guinea und Louisiaden & - & - \\
Salomon-Inseln & als australis & australis \\
Nord-Australien & - &
\end{tabular}

\section{Subgen. Macroglossus F. Cuvier.}

1825. F. Cuvier, Dents des Mammif. p. 40-41, 248.

Schwanzflughaut am Knie breiter als $4 \mathrm{~mm}$; Schneidezähne kürzer als der erste Molar; die mittleren stehen mindestens ebenso weit von einander wie die äusseren; zwischen dem ersten und zweiten Praemolar ist eine grössere Lücke als zwischen dem zweiten und dritten Praemolar, zuweilen nur im Unterkiefer; auf dem Gaumen befindet sich hinter sechs ungetheilten, in leichtem Bogen verlaufenden Querfalten eine kürzere, nicht bis zu den Gaumenrändern reichende, in der Mitte verdickte und zuweilen getheilte Querfalte. Auf dem Plagiopatagium wird der vom Ellenbogen bis zur Spitze des fünften Fingers verlaufende Strang von mindestens 10 Fascien ziemlich senkrecht durchschnitten.

Hab. Darjiling, Siam, Sumatra, Java, Borneo, Timor, Banda, Celebes, Philippinen, Amboina-Gruppe, Neu-Guinea, Bismarck-Archipel, Salomon-Inseln, Aru-Inseln. 


\section{Uebersicht der Arten:}

Zwischen den Nasenlöchern eine flache Grube, welche nicht bis zum Lippenrande reicht; die 7. Gaumenfalte ist vom gezähnelten Hinterrande des Gaumens viel weiter entfernt als die zweite Gaumenfalte von der dritten

Zwischen den Nasenlöchern eine tiefe, scharf begrenzte bis zum Lippenrande verlaufende Grube; die 7. Gaumenfalte ist nicht weiter vom gezühnelten Hintergrunde des Gaumens entfernt, als die zweite Gaumenfalte von der dritten.

Ausgewachsene Thiere mit verwachsenen Epiphysen der Fingergelenke haben eine Unterarmlänge von höchstens $39 \mathrm{~mm}$; der Vorderrand der 7. Gaumenfalte ist rechts und links von der Mitte stark eingebuchtet. . . . . . . . . .

Ausgewachsene Thiere mit verwachsenen Epiphysen der Fingergelenke haben eine Unterarmlänge von mindestens $40 \mathrm{~mm}$; der Vorderrand der 7. Gaumenfalte ist fast geradlinig .

M. minimus p. 96

M. nanus p. 98

M. lagochilus p. 97

1. M. minimus (E. Geoffroy St. Hilaire). - 1810. Pteropus minimus E. Geoffroy St. Hilaire von Java, Ann. Mus. d'Hist. Nat. XV, p. $97-98$. - 1821. Pteropus rostratus Horsfield von Java, Zool. Researches Java, Nr. 3, Tafel (Thier, Kopf von oben und von der Seite). - 1822. F. Cuvier und Is. Geoffroy St. Hilaire, Hist. Nat. Mammif. V. Fasc., 38, Taf. Kiodote (Thier). - 1825. Macroglossus minimus Dents des Mammif. p. 40-41, 248. - 1827. Pt. minimus Temminck, Monogr. Mammif. I, p. 191-194, Taf. 15, Fig. 25 (Gebiss von vorn), 26 (Schädel von der Seite), 27 (Unterkiefer von oben), 28 (Unterkiefer von unten), 29 (Schädel eines jungen Thieres von oben), 30 (Schädel eines jungen Thieres von der Seite), Taf. 16, Fig. 1 (Skelet), 2 (Becken und Schwanz). - 1827. Macroglossa kiodotes und $M$. Horsfieldii, beide von Java, andere Namen für Pt. minimus und rostratus, Lesson, Man. Mammal. p. 115. - 1838. Macroglossa minima Gray, Mag. Zool. Bot. II, p. 504. - 1869. Macroglossus minimus partim Fitzinger, Sitzb. Akad. Wien, LX, 1. Abth. Nov. p. 21-24. - 1878. Dobson, Cat. Chiropt. p. 96-97. 1887. Jentink, Cat. Ost. p. 268-269. - 1888. Jentink, Cat. Syst. p. 158-159. 1897. Trouessart, Cat. Mamm. p. 90.

\section{Hab. Java.}

Oldf. Thomas hat [Proc. Zool. Soc. 1888 p. 476] darauf aufmerksam gemacht, dass die Macroglossus von Java sich leicht durch ihre Grösse (45-48 mm Unterarmlänge), durch den verlängerten Gesichtstheil des Schädels und das Fehlen einer tiefen und deutlichen Grube über die Oberlippe von allen anderen Macroglossus-Exemplaren erkennen lassen. Auch Jentink (Not. Leyd. Mus. XIX, 1897 p. 51-52) bestätigt die Verschiedenheit der Macroglossus von Java und hält es für wahrscheinlich, dass unter den mit einer Lippenfurche versehenen Macroglossus vielleicht $\mathrm{mehr}$ als eine Art versteckt sei.

Ich beschränke den Namen $M$. minimus Geoffr. auf die Form von Java, welche genügend gekennzeichnet wird durch folgende Diagnose:

Macroglossus, labio superiore haud sulcato, radio: 45 ad $48 \mathrm{~mm}$, facie valde elongata.

Zwischen den Nasenlöchern ist eine flache Grube zu erkennen, welche aber nicht bis auf die Oberlippe reicht. Die 7. Gaumenfalte ist von dem gezähnelten 
Hinterrande des Gaumens viel weiter entfernt als die zweite Gaumenfalte von der dritten. Der Unterarm derjenigen Thiere, bei welchen die Epiphysen an den Fingergelenken mit den Phalangen-Enden bereits verwachsen sind, hat eine Länge von mindestens $45 \mathrm{~mm}$. Die Molarenreihe ist im Oberkiefer länger ais $7 \mathrm{~mm}$.

[B. M.] ㅇ. Java, Arendt; ðð pull. Java, Nagel; 우 Java, Bernstein.

2. M. lagochilus Matschie spec. nov. - 1888. M. minimus Jentink von Amboina, Cat. Syst. p. 159.

Macroglossus, labio superiore sulcato; radio: 40,5 ad $44,5 \mathrm{~mm}$; facie elongata.

\section{Hab. Buru, Amboina.}

Zwischen den Nasenlöchern verläuft eine tiefe, scharf begrenzte Grube bis zum Lippenrande. Die 7. Gaumenfalte ist von dem gezähnelten Hinterrande des Gaumens nicht weiter entfernt als die zweite Gaumenfalte von der dritten. Ihr Vorderrand ist fast geradlinig. Der Unterarm derjenigen Thiere, bei welchen die Epiphysen an den Fingergelenken mit den Phalangen-Enden bereits verwachsen sind, hat eine Länge von 40,5 bis $44,5 \mathrm{~mm}$. Die Molarenreihe ist kürzer als $7 \mathrm{~mm}$. Die Schnauze ist ziemlich lang. Auch in der Färbung scheint ein Unterschied vorhanden zu sein; die Unterseite ist sehr hell, fast weiss. Museum.

Als Typus der neuen Abart gelte das Exemplar von Buru im Berliner

\section{[B. M.] §̋ Buru, Bleeker; 우 Amboina, Müller \& Macklot.}

Anmerkung: Ob die Exemplare aus Sumatra eine besondere Abart darstellen oder mit der auf Java vorkommenden Abart vereinigt werden müssen, weiss ich nicht. Jentink giebt (Weber, Zool. Ergebn. Reise Niederl. Ost-Indien I, 1, 1890 , p. 127) für ein $\sigma^{\top}$ die Unterarmlänge von $47 \mathrm{~mm}$ an. Modigliani nennt (Aṇ. Mus. Genova 2. ser. VII, 1889, p. 242) für Sitoli auf Nias M. minimus.

Wie die Macroglossus in Hinterindien aussehen, darüber hat Thomas sich nicht genauer erklärt. Er führt aber die von Fea gesammelten Birma-Stücke (o Taho, + Biapo, An. Mus. Genova 2. ser. VII, 1892 p. 242) als M. minimus an, woraus hervorzugehen scheint, dass sie, wie die Javaner, keine Lippenfurche hatten. Anderson zählt (Cat. Mamm. Ind. Mus. I. 1881, p. 107) ein Stück aus Darjiling und ein anderes aus Siam auf.

Ob die Macroglossus von Borneo, bei denen übrigens die Oberlippe bis zum Lippenrande gefurcht ist, mit $M$. lagochilus übereinstimmen, kann ich nicht entscheiden, da wir nur ein einziges, noch dazu junges Exemplar von dort besitzen, welches in Sarawak gesammelt worden ist. Sein Unterarm ist nur $38 \mathrm{~mm}$ lang, die Epiphysen der Finger sind aber noch nicht verwachsen. Die Unterseite des Körpers ist sehr hell. Die Gaumenfalten gleichen denen des Buru-Exemplares, nur hat die 7. Falte in der Mitte einen vorderen Einschnitt. Jentink sagt (Not. Leyd. Mus. XIX, 1897, p. 51), dass bei einem 우 von Roema Manoeal am Südabhang des Kenepai-Berges der Unterarm $42 \mathrm{~mm}$ lang sei.

Ebenso wenig wissen wir über die Macroglossus von Celebes und von den Philippinen. Das Berliner Museum hat 2 Exemplare aus Celebes, leider aber ist das eine ausgestopft und das andere sehr jung. Im Schädelbau und in der Form der Gaumenfalten gleichen beide dem Borneo-Stücke sehr gut. Die Unterseite ist aber nicht so hell, sondern nur wenig heller als der Rücken. Casto Fledermäuse. 
de Elera (Cat. Sist. Fauna Filipinos I, 1895, p. 7-8) giebt für Panay, Samar und Cuyo diesen Flederhund an.

Jentink erwähnt (Not. Leyd. Mus. 1883 p. 174 und 1888 Cat. Syst. p. 159) M. minimus von Nord-Celebes, Thomas (Trans. Zool. Soc: XIV pt. VI p. 385) von Negros in den Philippinen und giebt an (Proc. Zool. Soc. 1888, p. 476), dass durch Cuming und Everett weitere Exemplare von den Philippinen im British Museum seien. Von der Halmahera-Gruppe scheint Macroglossus noch nicht bekannt geworden zu sein, auch über den Timor-Macroglossus wissen wir nur durch Temminck (Monogr. Mammal. II, p. 97), dass er sehr langköpfig und den Javanern äusserst ähnlich ist.

Ob das Thier auf Banda dem Amboina-Macroglossus gleicht oder eine besondere Form darstellt, darüber giebt Temminck keinerlei Andeutungen.

[B. M.] 오 Sarawak, durch Marquis Doria; ơ.Menado, Celebes, von Faber; ot pull. Menado, Celebes, A. B. Meyer.

3. II. nanus Matschie spec. nov. - 1885. M. minimus Pagenstecher, Jahrb. Wiss. Anst. Hamburg, II, p. 126-128, Tafel Fig. 3 (Gaumen), 3 a (Oberlippe und Zunge), 3 b (Flughautansatz am Fuss). - 1888. M. minimus Jentink von NeuPommern, Jentink, Cat. Syst. p. 159. - 1888. M. australis Ptrs. von Mysol und Neu-Lauenburg, Thomas, Proc. Zool. Soc. London, p. 476.

Macroglossus, labio superiore sulcato, radio $37-39,5 \mathrm{~mm}$; facie haud elongata.

\section{Hab. Bismarck-Archipel, Neu-Guinea, Aru-Inseln, Salomon-Inseln (?)}

Zwischen den Nasenlöchern verläuft eine tiefe, scharf begrenzte Grube bis zum Lippenrande. Die 7. Gaumenfalte, welche in der Mitte nach vorn und hinten etwas verbreitert ist, liegt von dem gezähnelten Hinterrande des Gaumens nicht weiter entfernt als die zweite Gaumenfalte von der dritten; ihr Vorderrand ist rechts und links von der Mitte stark eingebuchtet. Der Unterarm ist nicht länger als $39,5 \mathrm{~mm}$. Die Molarenreihe ist kürzer als $7 \mathrm{~mm}$. Die Schnauze ist ziemlich kurz. Die Unterseite des Körpers ist weisslich; auch auf dem Rücken sind die Haare in der unteren Hälfte weisslich.

Als Typus dieser Abart gelte ein ơ von Lamellana, Neu-Pommern, Nr. 9308 des Berliner Museums, welches Herr Professor Dr. Dahl gesammelt hat.

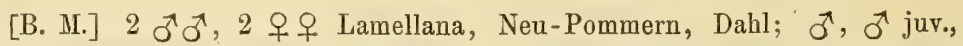
2 우오 Neu-Pommern, Finsch; 우 Andai, Neu-Guinea, d'Albertis; तત Aru-Inseln, Wokam, von Rosenberg.

Vielleicht gehören die von Aola, Shortland-Insel durch Thomas beschriebenen Stücke (Proc. Zool. Soc. London 1888, p. 476) hierher. Mit grosser Wahrscheinlichkeit ist auch Pagenstechers Macroglossus (s. oben 1885) zu M. nanus zu stellen.

\section{Subgen. Syconycteris Matschie subgen. nov.}

Schwanzflughaut am Knie höchstens $3 \mathrm{~mm}$ breit; Schneidezähne länger als der erste Molar, die mittleren stehen näher zusammen als die äusseren; zwischen dem ersten und zweiten Praemolar ist kein grösserer Zwischenraum als zwischen den übrigen Zähnen. 
Auf dem Gaumen befinden sich sieben ungetheilte Querfalten, die alle oder fast alle in der Mitte winklig nach vorn geknickt sind. Auf dem Plagiopatagium wird der vom Ellenbogen bis zur Spitze des fünften Fingers verlaufende Strang von höchstens 9 grösseren Fascien ziemlich senkrecht durchschnitten.

Typus: Macroglossus australis Peters.

Neu-Guinea.

Hab. Nord- und West-Australien, Bismarck-Archipel, Louisiaden, Aru-Inseln,

\section{Uebersicht der Arten:}

Unterarm bei erwachsenen Thieren länger als $45 \mathrm{~mm}$. . . . . $M$. crassus p. 100

Unterarm bei erwachsenen Thieren kürzer als $44 \mathrm{~mm}$ :

Die Muffel ist am Lippenrande so breit wie die Entfernung des

Nasenloches vom Lippenrande . . . . . . . . .

M. australis p. 99

Die Muffel ist am Lippenrande viel breiter als die Entfernung

des Nasenloches vom Lippenrande:

Unterarm bei erwachsenen Thieren länger als $41 \mathrm{~mm}$ - M. papuanus p. 99

Unterarm bei erwachsenen Thieren kürzer als $39 \mathrm{~mm}$. M. finschi p. 100

1. II. australis Peters. - 1867. Macroglossus minimus var. australis Peters von Rockhampton in Nordost-Australien, Monatsb. Akad. Berlin, p. 13, Anmerkung 1. - 1887. M. minimus Collett, Zool. Jahrb. II, p. 845.

Peters Exemplar von Rockhampton gehört zu Syconycteris, nicht zu Macroglossus s. str. Thomas' Beschreibung von Carponycteris crassa lässt sich auf dieses Stück sehr gut anwenden; nur ist $M$. australis kleiner als $M$. minimus, sein Unterarm ist viel kürzer als $47 \mathrm{~mm}$, die Reihe der oberen Molaren ist 5,5 mm lang. Was Thomas (Proc. Zool. Soc. London 1888 p. 476) M. australis nennt, das hat nur. mit Macroglossus im engeren Sinne etwas zu thun. M. australis unterscheidet sich von unseren Syconycteris-Stücken aus Papuasien durch die grössere Höhe der Unterlippe unterhalb des Nasenlochs; auf dem Gaumen ist die letzte Querfalte gezähnelt und geradlinig, während die vorletzte stumpfwinklig nach vorn gebogen und in der Mitte etwas weiter von ihr entfernt ist, als von der vorhergehenden. Ich habe deshalb dem Macroglossus aus Nordwest-Neu-Guinea einen neuen Namen beigelegt. Ob die Exemplare von Aru und vom Bismark-Archipel mit denjenigen aus Nordwest-Neu-Guinea in jeder Beziehung übereinstimmen, das bleibt zu untersuchen. Unser Material reicht dazu nicht aus.

Collett erwähnt einen Macroglossus von Mackay, etwas nördlicher als Rockhampton; er hat einen $41 \mathrm{~mm}$ langen Unterarm und die inneren oberen Schneidezähne convergiren. Hiernach wird es sehr wahrscheinlich, dass Collett's Exemplar zu Syconycteris gerechnet werden muss.

Abb. Taf. [14], Fig. 1 (Thier), 2. (Kopf), 3 (Flughautansatz am Fusse), 4 (Schädel von der Seite), 5 (Schädel von oben), 6 (Schädel von unten), 7 (Gebiss von vorn), 8. (Gebiss von der Seite), 9 (Gaumenfalten), 10 (Unterkiefer von oben).

[B. M.] 0 Rockhampton, Krefft.

2. M. papuanus Matschie spec. nov.

Syconycteris, radio breviore quam $44 \mathrm{~mm}$, rhinario subtus latiore quam labium superius infra nares, molarium serie $6 \mathrm{~mm}$ aliquantum superante. 
Hab. Nordwest-Neu-Guinea (Andai, Sorong), vielleicht auch die Aru-Inseln.

Als Typus dieser Art habe ich ein $\widehat{0}$ von Andai zwischen Dorei und den ArfakBergen im Osten der Nordwest-Halbinsel von Neu-Guinea gewählt.

Die drei Exemplare von Andai und Sorong (Westküste der Nordwest-Halbinsel) haben eine Unterarm-Länge von $41,5-43 \mathrm{~mm}$. Die nackte Muffel ist am Lippenrande viel breiter als die Entfernung des unteren Randes des Nasenloches vom Lippenrande. Die Reihe der oberen Molaren ist länger als $6 \mathrm{~mm}$. Auf dem Gaumen ist die letzte Querfalte weiter von der vorletzten entfernt, als diese von der vorhergehenden, und in der Mitte stumpfwinklig nach vorn gebogen. Die Molaren sind verhältnissmässig breit und kräftig.

Ein Stück von den Aru-Inseln, welches das Berliner Museum besitzt, hat eine Unterarmlänge von $41,5 \mathrm{~mm}$, die Molarenreihe ist bei ihm $6,9 \mathrm{~mm}$ lang, die Molaren sind breit und kräftig. Ich würde diese Syconycteris ohne weiteres zu papuanus ziehen, wenn nicht die Gaumenfalten einen Unterschied gegen die drei Stücke von Nordwest-Neu-Guinea zeigten. Die letzte Querfalte steht zwar auch weiter von den übrigen entfernt als diese unter sich, verläuft aber fast geradlinig, nur ganz schwach nach vorn gebogen und ist in der Mitte nicht stumpfwinklig geknickt. Da aber nur ein Exemplar vorliegt, so begnüge ich mich damit, auf diese Eigenthümlichkeit der Gaumenfalten bei der Aru-Syconycteris hingewiesen zu haben.

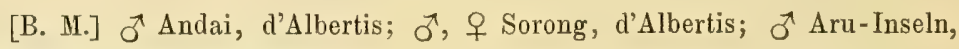
Wokam, von Rosenberg.

3. M. finschi Matschie spec. nov.

Syconycteris, radio breviore quam $40 \mathrm{~mm}$, rhinario subtus latiore quam labium superius infra nares, molarium serie vix 5,6 mm longa.

Hab. Neu-Pommern im Bismarck-Archipel.

Als Typus dieser Art hat ein von Herrn Dr. Finsch auf Neu-Pommern gesam. meltes of zu gelten. Ich erlaube mir, dem Sammler diese Art zu widmen in der Erinnerung an das schöne Material von Fledermäusen, welches das Berliner Mfuseum Herrn Dr. Finsch verdankt.

Nur ein einziges Exemplar liegt vor; es zeigt aber so grosse Verschiedenheiten von den übrigen Syconycteris-Stücken, dass ich einer Trennung des Exemplares aus Neu-Pommern von den Neu-Guinea-Syconycteris nicht aus dem Wege gehen darf.

Die nackte Muffel ist noch etwas breiter als bei $M$. papuanus; die Reihe der oberen Molaren ist nur 5,6 mm lang, die Molaren sind auffallend klein, schwach und schmal; der erste echte obere Molar ist nur $0,5 \mathrm{~mm}$ breit. Auf dem Gaumen sind die letzten beiden Querfalten geradlinig und von den übrigen nicht weiter entfernt als von einander. Der Unterarm ist nur $37,9 \mathrm{~mm}$ lang.

[B. MI.] $\widetilde{\jmath}$ Neu-Pommern, Finsch.

4. M. crassus Thos. - 1895. Carponycteris crassa Thomas von dem FergussonIsland, De Entrecasteaux-Gruppe, Nov. Zool. II, p. 163. - 1896. Thomas, Nov. Zool. III, p. 526. - 1897. Thomas, Ann. Mus. Civ. Genova, 2. ser. vol. XVIII, p. 608. - 1897. Macroglossus crassus Trouessart, Cat. Mamm. Nov. Ed. I, p. 90. 
Hab. Fergusson Island in der D'Entrecasteauz-Gruppe (Thomas 1895); Trobiand (Kiriwana-) und Woodlark- (Murua-) Inseln (Thomas 1896); Ighibirei, südlich vom Owen-Stanley-Gebirge am Kemp Welch-Flusse in SüdostNeu-Guinea (Thomas 1897).

\section{Gattung. Megaloglossus Pagenstecher.}

1885. Megáloglossus Pagenstecher, Zool. Anzeig. VIII, p. 245, Jahrb. Wiss. Anst. Hamburg, II, p. 125-128. - 1887. Thomas, Proc. Zool. Soc. London p. 324. - 1891. Trygenycteris Lydekker in Flower und Lydekker, Introduction to the Study of Mammals, p. 655.

Sehr kleine Flughunde ohne deutlichen Schwanz: Die Schwanzflughant ist in der Analgegend von der Behaarung verdeckt. Zeigefinger mit Kralle. Der Rand des Ohres bildet einen ovalen Ring. Die Flughaut setzt sich an die Körperseiten und mit zwei Fältchen an die Wurzeln der zweiten und dritten Zehe an. Der Metacarpus des Mittelfingers ist ungefähr so lang. wie der Zeigefinger mit der Kralle. Die Innenränder der Nasenlöcher sind nicht aufgeworfen. Die Schnauze ist sehr lang und schmal; zwischen den Nasenlöchern verläuft eine Längsfurche bis zum Lippenrande. Die Muffel ist am Lippenrande sehr breit und an den Seiten convex.

Gebiss: $\frac{2.1 .3 .2}{2.1 .3 .3}$. Die Zähne ausser den Eckzähnen sind schwach. Im Oberkiefer haben die Eckzähne vorn eine Furche; die Schneidezähne stehen im Trapez; der erste Praemolar ist auffallend klein und vom zweiten Praemolar etwas weiter entfernt als dieser vom dritten. Im Unterkiefer sind die Eckzähne nach hinten gerichtet. Das Palatinum reicht weit über die Zahnreihe hinaus. Auf dem Gaumen stehen 6 Querfalten, von denen die letzten beiden getheilt sind. Die Zunge ist sehr lang; die Papillen sind denen von Melonycteris ähnlich. Die Saugwarzen liegen in der Höhe des Ellenbogens an den Brustseiten.

Ueber die Lebensweise der Langzungen-Fledermäuse ist nicht viel bekannt. Büttikofer fing in Liberia ein Exemplar in einer Negerhütte, Sjöstedt ein solches in dichtem Gebüsch, wo es an einem Zweige gehangen hatte.

Lydekker's Vorschlag, den Namen Megaloglossus zu Gunsten von Trygenycteris zu verwerfen, weil es schon eine Megaloglossa 'giebt, vermag ich nicht zu folgen. Ich nehme mit der Deutschen Zoologischen Gesellschaft etymologisch gleich abgeleitete, aber durch verschiedene Endsilben als verschieden gekennzeichnete Namen an.

Typus: Megaloglossus woermanni Pagenstecher.

Hab. West-Afrika (Liberia, Kamerun, Gabun, Congo).

Nur eine Art: M. woermanni Pagenstecher. - 1885. Megaloglossus Woermanni Pagenstecher von der Sibange-Farm bei Gabun, West-Afrika, Zool. Anzeig. VIII, p. 245. - 1885. Pagenstecher, Jahrb. Wiss. Anst. Hamburg, II, p. 125128, Tafel, Fig. 1 (Thier), 1 a (Oberkiefer von der Gaumenseite), 1 b (Oberlippe und Zunge), 1c (Flughautansatz am Fuss). - 1887. Thomas, Proc. Zool. Soc. London, p. 324. - 1888. Jentink, Not. Leyd. Mus. X, p. 53. - 1888. Jentink, Cat. Syst. p. 159. - 1889. Noack, Zool. Jahrb. IV, p. 209-213, Taf. 5, Fig. 56 
(Schädel und Unterkiefer von der Seite), 57 (Schädel von oben), 58 (Gaumen). 1895. Sjöstedt, K. Sv. Vet. Akad. Handl. Bd. 27, Nr. 1, p. 119. - 1895. Sjöstedt, Bih. Kgl. Sv. Vet. Akad. Handl. Bd. 21, Afd. IV, Nr. 1, p. 1-7, Taf. (Thier von vorn und hinten, Becken mit der Schwanzwirbelsäule). - 1897. Sjöstedt, Mitth. Deutsch. Schutzgeb. X, p. 7. - 1897. Sjöstedt, Bih. K. Sv. Vet. Akad. Handl. Bd. 23, Afd. IV, Nr. 1, p. 16, 46. - 1897. Trouessart, Cat. Mamm. Nov. Ed. p. 90.

Hab. Liberia (Schieffinsville am Junk River, Jentink), Kamerun (Ekundu, Sjöstedt), Gabun (Sibange-Farm, Pagenstecher), Congo (Netonna, Noack). 


\section{Druckfehler, Berichtigungen und Zusätze.}

Seite 5 , Zeile 13 von oben ist statt $\frac{4,1,3,2}{4,1,3,3}$ zu lesen $\frac{2,1,3,2}{2,1,3,3}$.

" $9, \quad, 16$ von oben sind die Klammern bei „Eschscholtz" zu streichen.

" 9, " 3 von unten sind die Klammern bei "Temminck" zu streichen.

"11, " 9 von unten sind hinter Bismarck-Archipel die Worte: Grange-Inseln (Thomas, Ann. Mus. Civ. St. Nat. Genova XIX (38) 1897 p. 608)" beizufügen.

"12,, 10 von oben sind die Worte: „- 1897. Thomas, Ann. Mus. Civ. St. Nat. Genova XIX (38) p. 608" beizufügen.

"15, „ 7 von oben ist "Hermann" hinter Pt. celaeno in Klammern zu schliessen.

"15, „ 19 von oben sind hinter 316 die Worte: „- 1897. Jentink, Not. Leyd. Mus. XIX p. 49 " beizufügen.

"23, " 4 von unten sind hinter „p. 5" die Worte: „- 1881. Pt. macklotii Peters und Doria, Ann. Mus. Civ. Genova p. 689" einzufügen.

"24, " 5 von oben sind hinter "p. 82" die Worte: "- 1897. Thomas, Ann. Mus. Civ. Genova XIX (38) p. 608" einzufügen.

"26, " 15 von unten sind hinter 148-149 die Worte: „-? Pt. medius Gerrit S. Miller jr., Proc. Acad. Philadelphia 1898, p. 316“ einzufügen.

"29, " 15 von unten ist vor und hinter „Linné" je eine Klammer einzufügen.

"29, " 15 von unten ist zu lesen statt "vanpyrus" "vampyrus".

"32, " 5 von oben ist einzufügen „Welcher Abart Hartert's Pt. temmincki von Alor zugehört (Nov. Zool. V, 1898 p. 456) bleibt vorläufig zweifelhaft".

"32, „15 von unten lies statt „eapistrata" „capistratus".

" 42, " 13 von oben ist "Halowell“ hinter "haldemani“ in Klammern zu schliessen.

"44, " 18 von unten ist "Ogilby" hinter macrocephalus in Klammern zu schliessen.

"53, " 17 von unten ist "Rüpp." hinter schoensis in Klammern zu schliessen.

"54, " 1 von oben ist "Temminck" hinter labiatus in Klammern zu schliessen.

"55, " 13 von unten ist "Ogilb." hinter gambianus in Klammern zu schliessen. 


Die zoogeographischen Gebiete der aethiopischen Region.

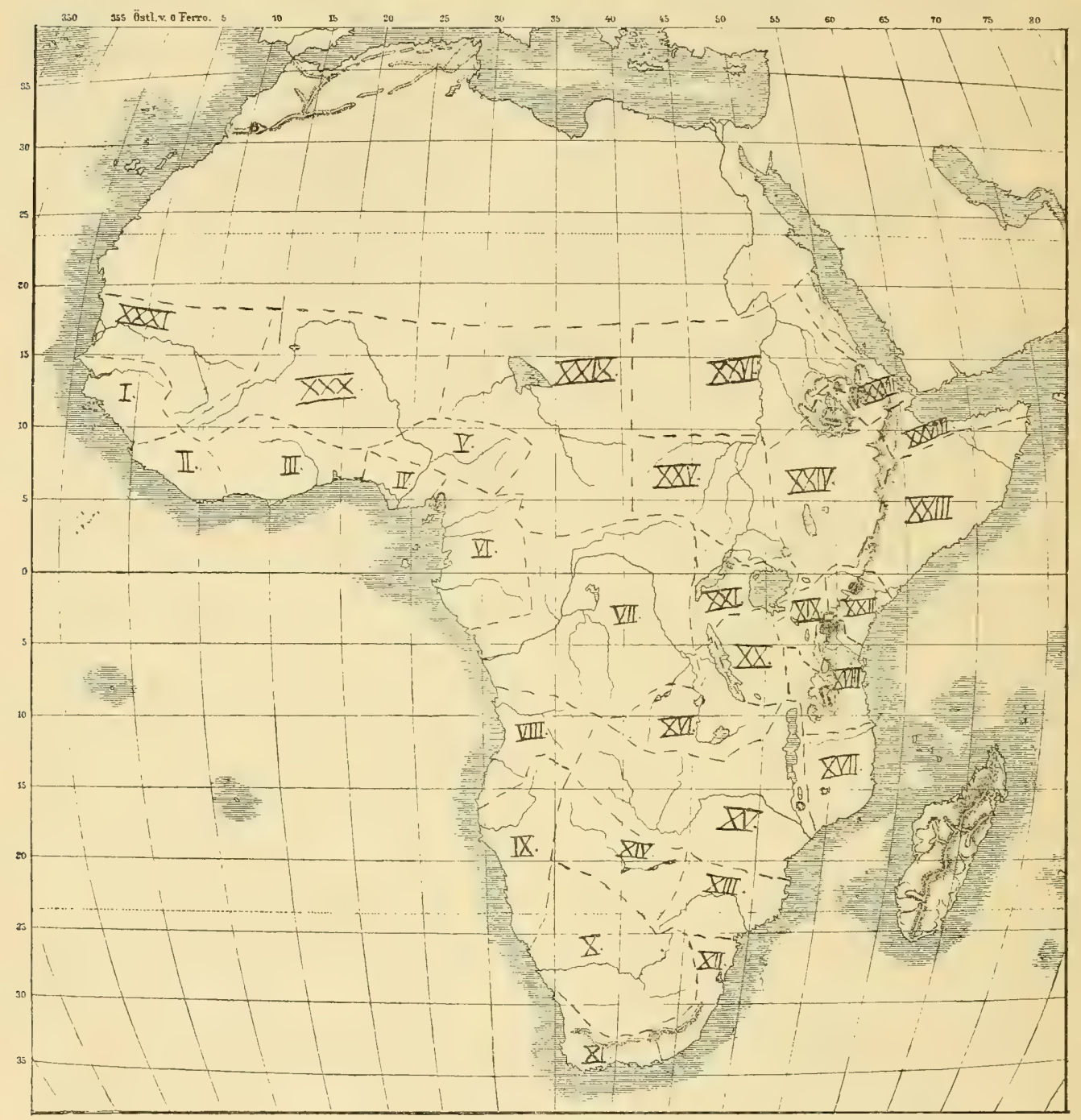

I. Gambia.

II. West-Guinea.

III. Mittel-Guinea.

IV. Unterer Niger.

V. Benue.

VI. Nieder-Guinea.

VII. Congo.

VIII. Loanda.

IX. Benguella.

$\mathrm{X}$. Orange.
XI. West-Capland.

XII. Ost-Capland.

XIII. Limpopo.

XIV. Ngami.

$\mathrm{XV}$. Zambese.

XVI. Hero.

XVII. Mossambik.

XVIII. Zanzibar-Küste.

XIX. Massai - Land.

XX. Malagarasi.
XXI. Seeen - Gebiet.

XXII. Ukambani.

XXIII. Somali.

XXIV. Rudolf-See.

XXV. Gazellen-Fluss.

XXVI. Bahr el Abiad.

XXVII. Erythraea.

XXVIII. Berbera-Küste.

XXIX. Tschad-See.

XXX. Oberer Niger.

XXXI. Senegal. 


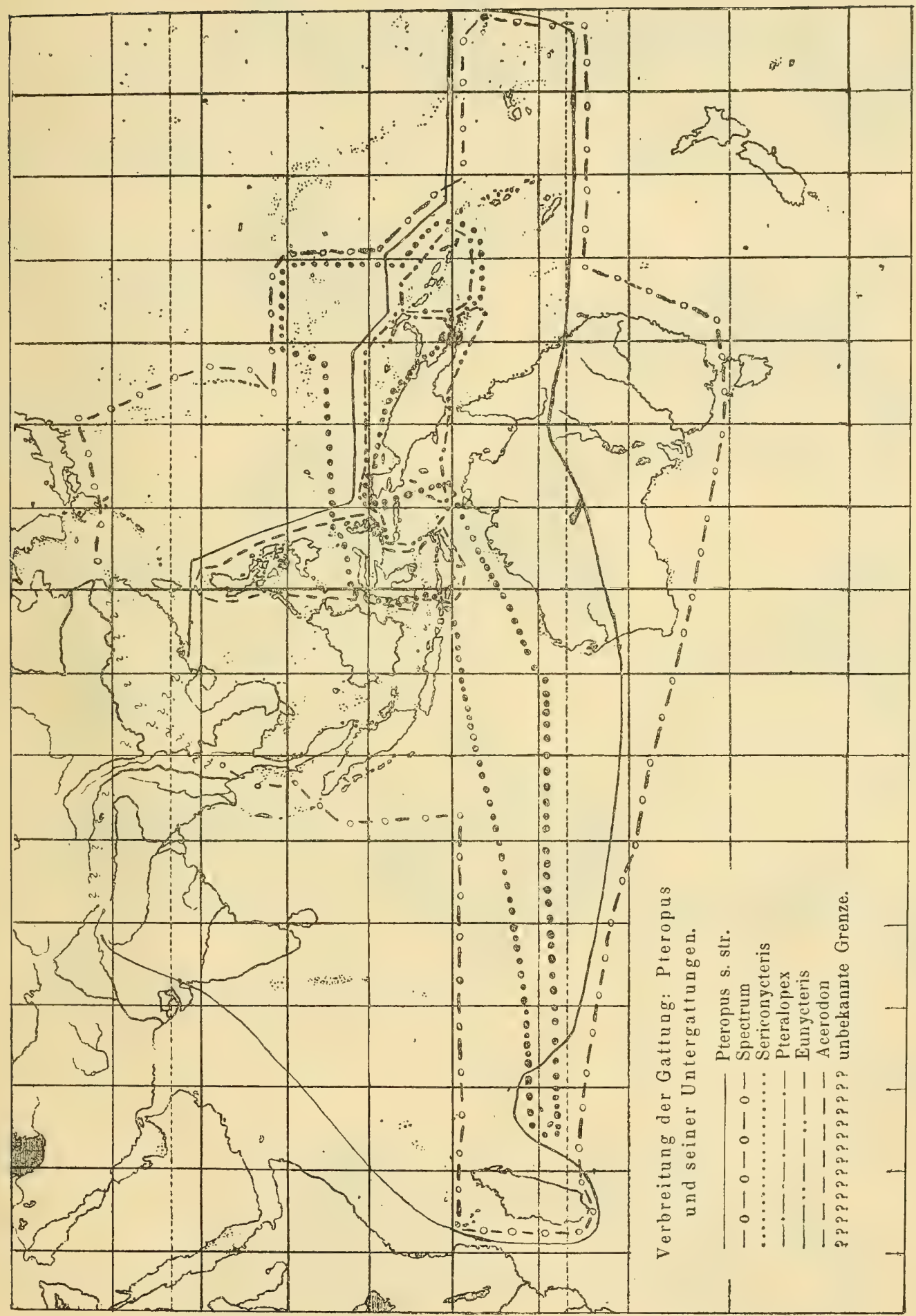





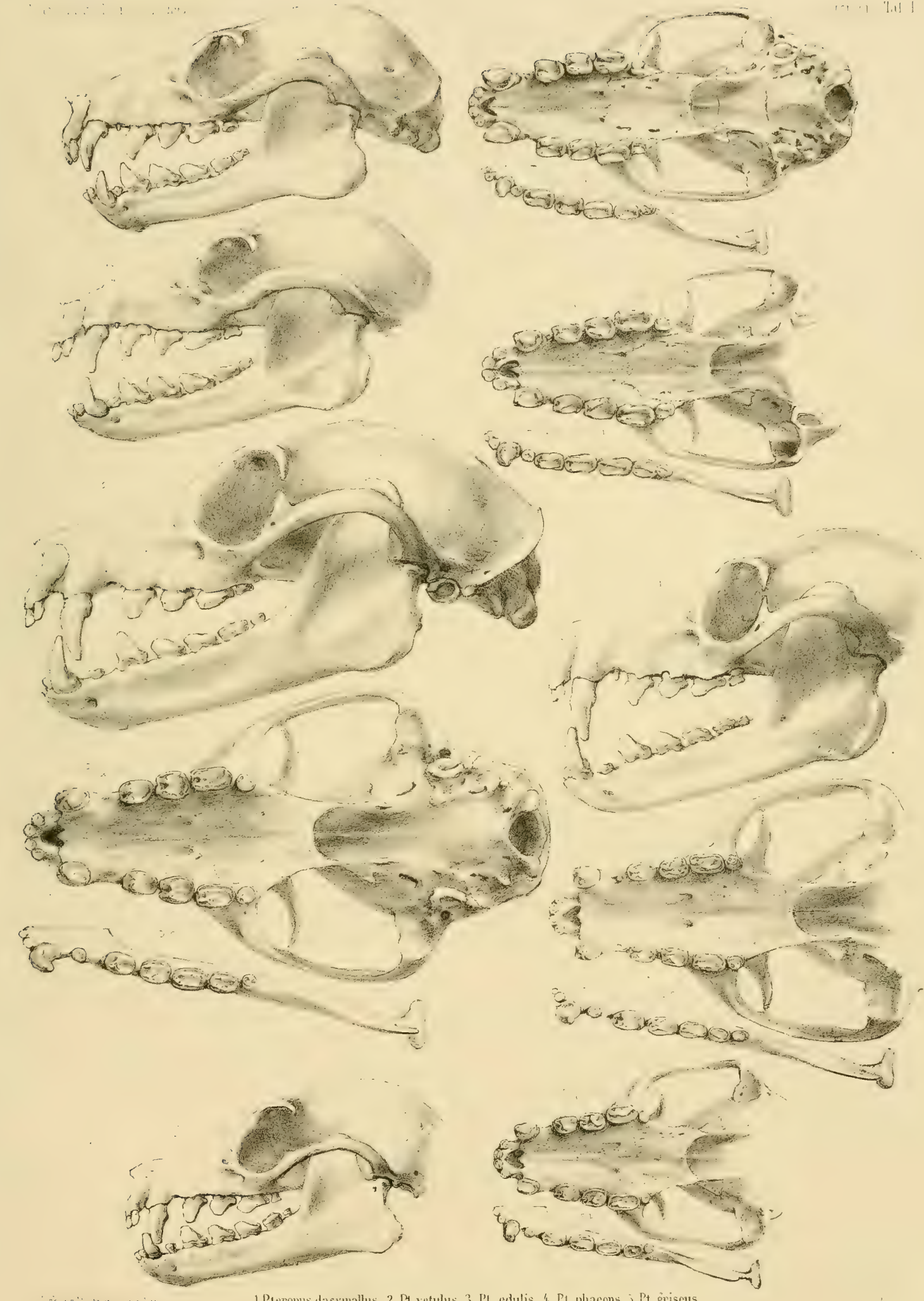





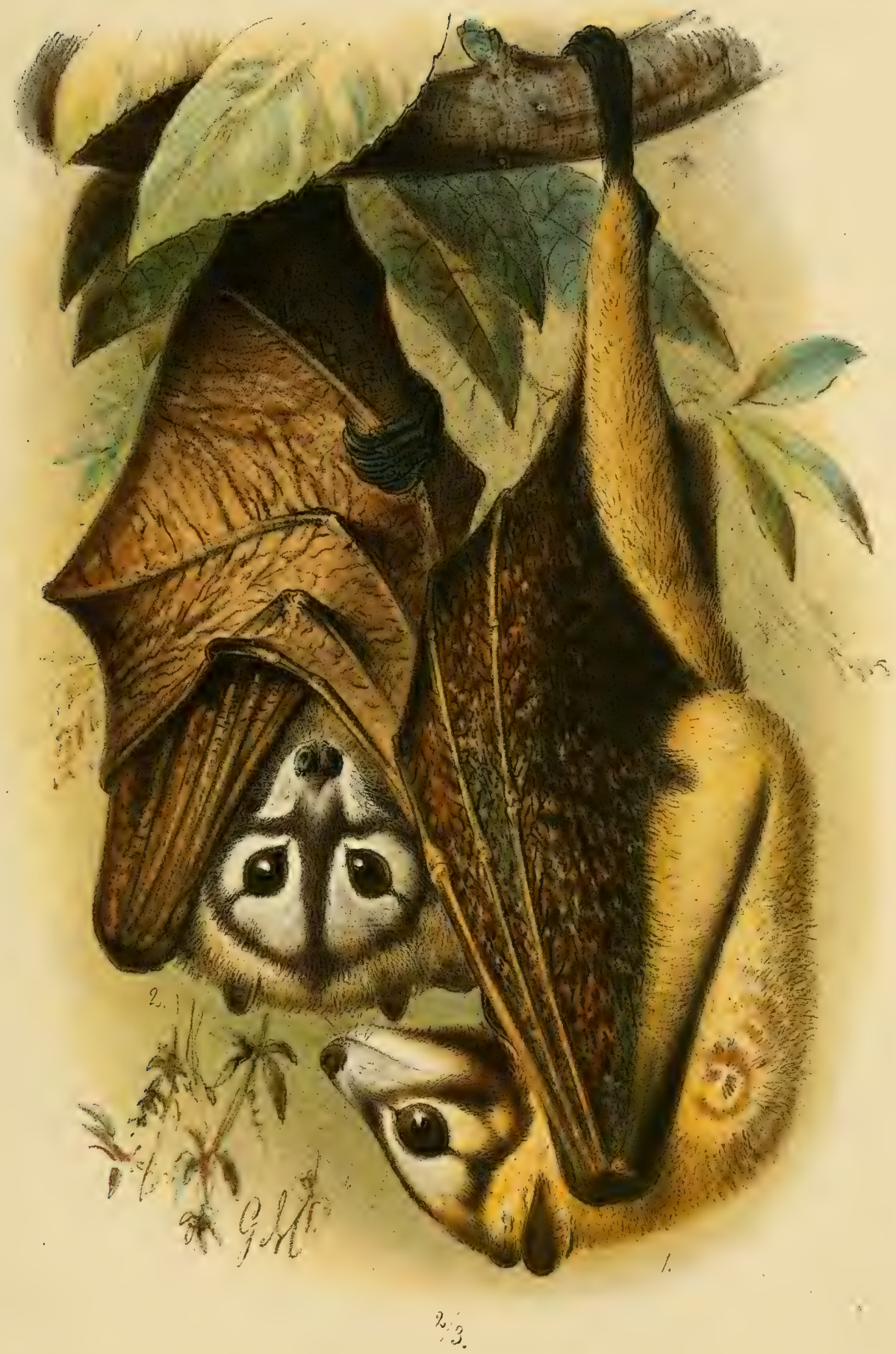

Pteropus capistratus Ptrs 1Mas 2Fem. 


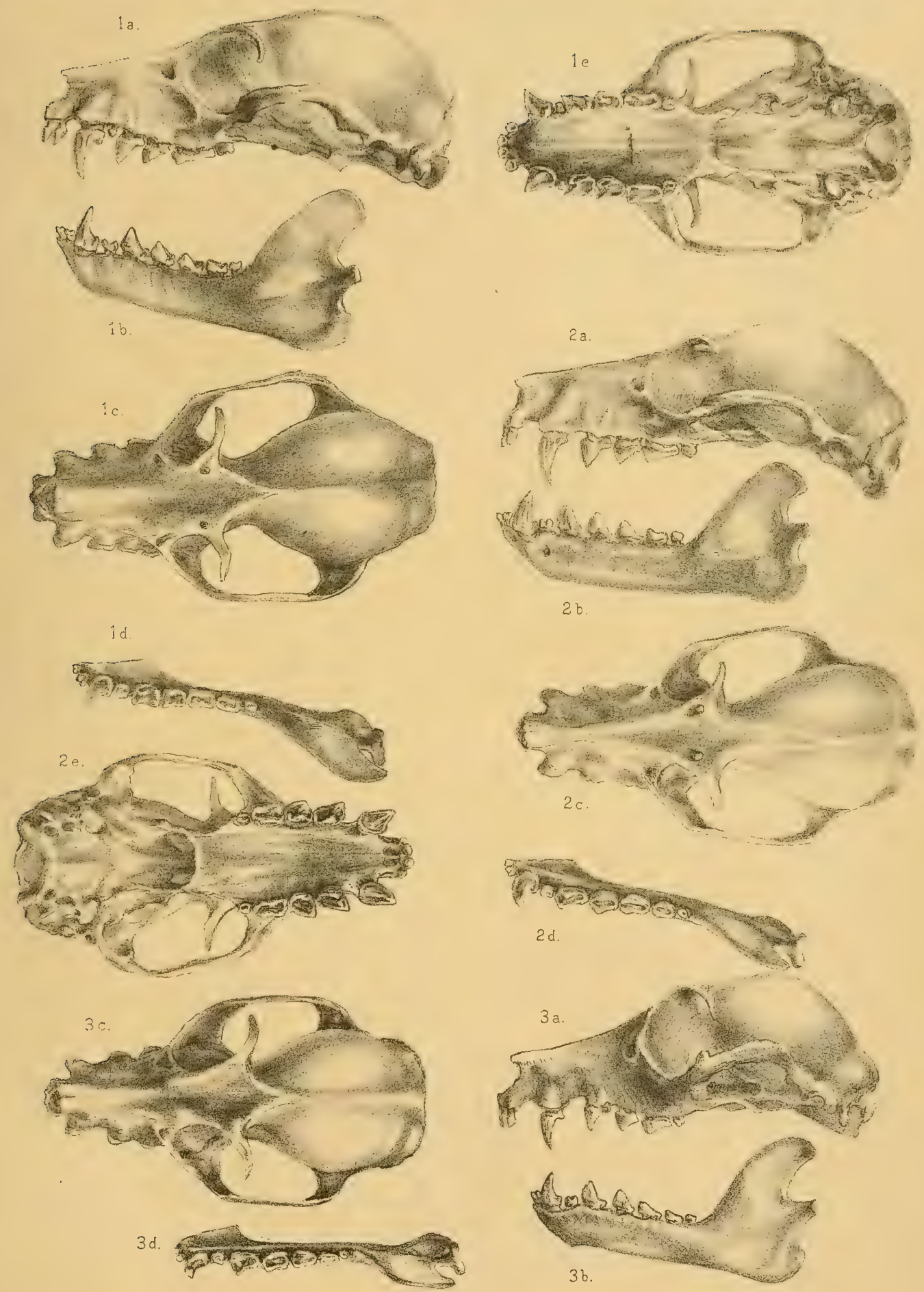

1.Pteropus samoensis. 2.Pteropus admiralitatum. 3.Pteropus capistratus. 


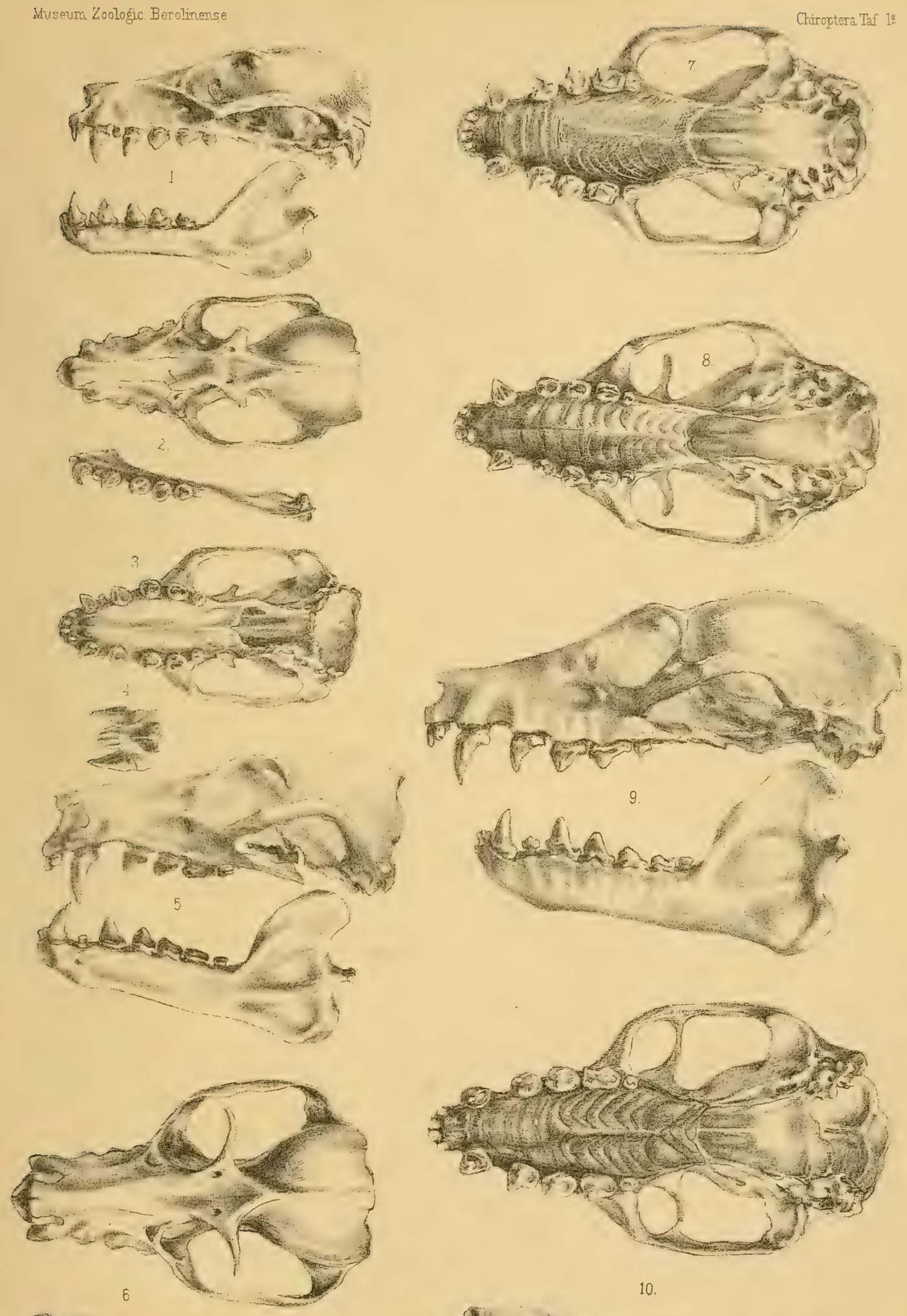

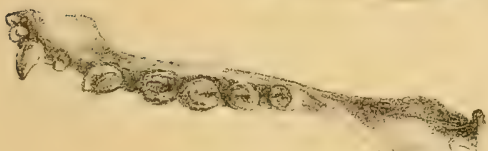

10.

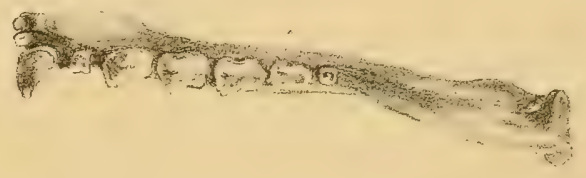

1-4. Styloctenium wallacei. 5-6. Pteropus ocularis. \%. Pt.celebensis. 8.Pt.assamensis 9-10. Pt. livingstoni. 



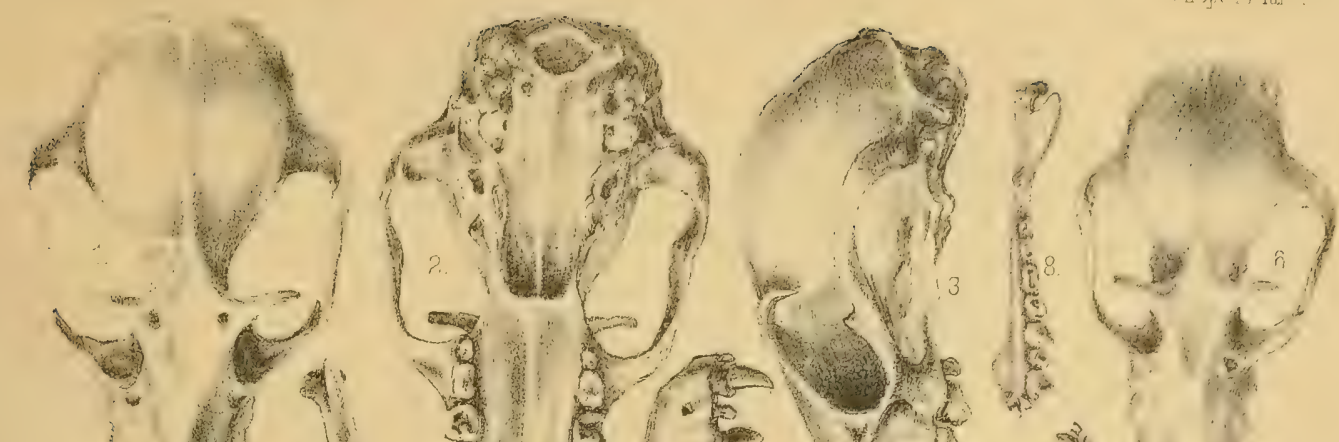

(1) 10.2 . y (a)

1.

再

हु

19

(6). है

- 8

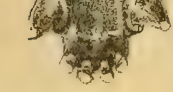

(V)
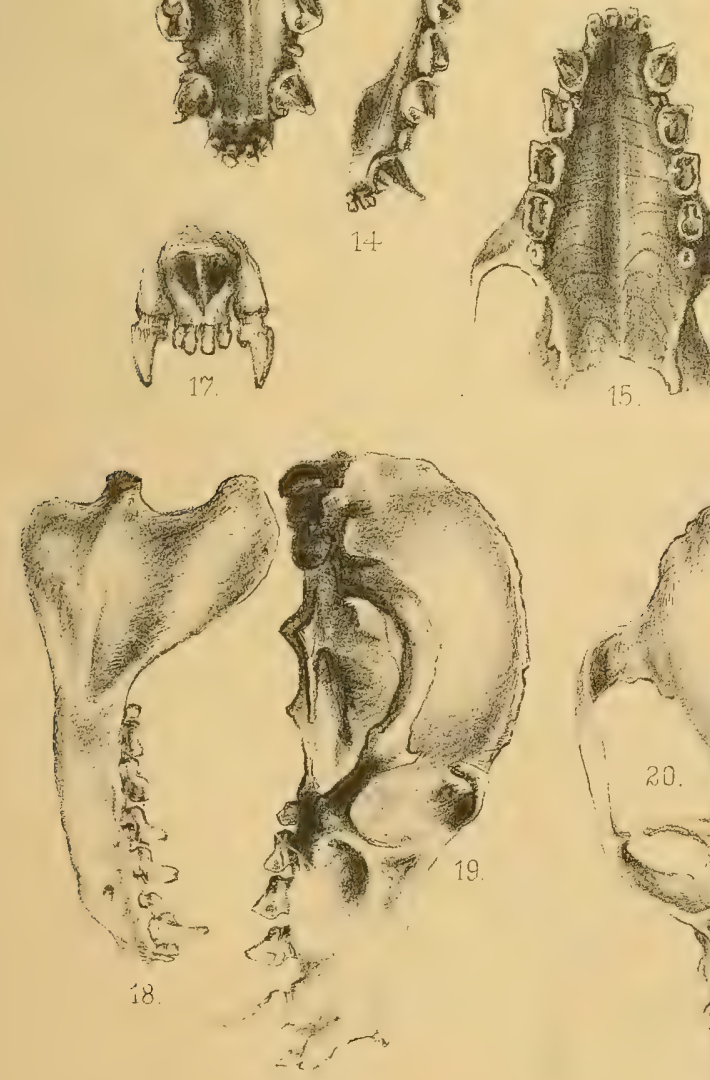

(1)

3.

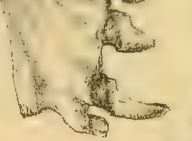

at

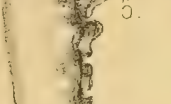





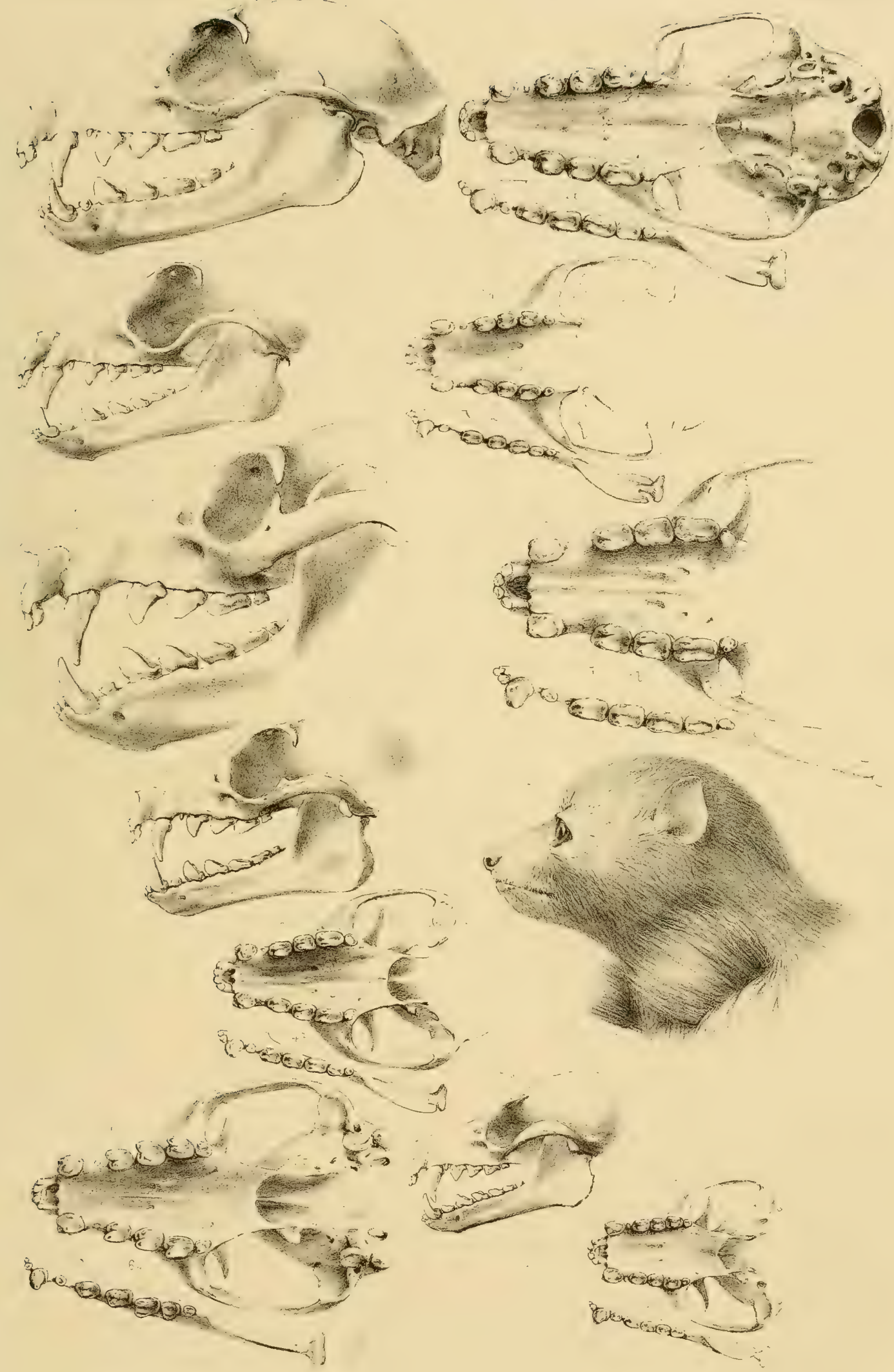

1 Pletopus alecto. 2 Pt.macrotis.3 Pt.melanopogon var aruensis. 4 Pt.Temmindkii 5 Pt molossinuls. 6 Pt. Hadklotii. 



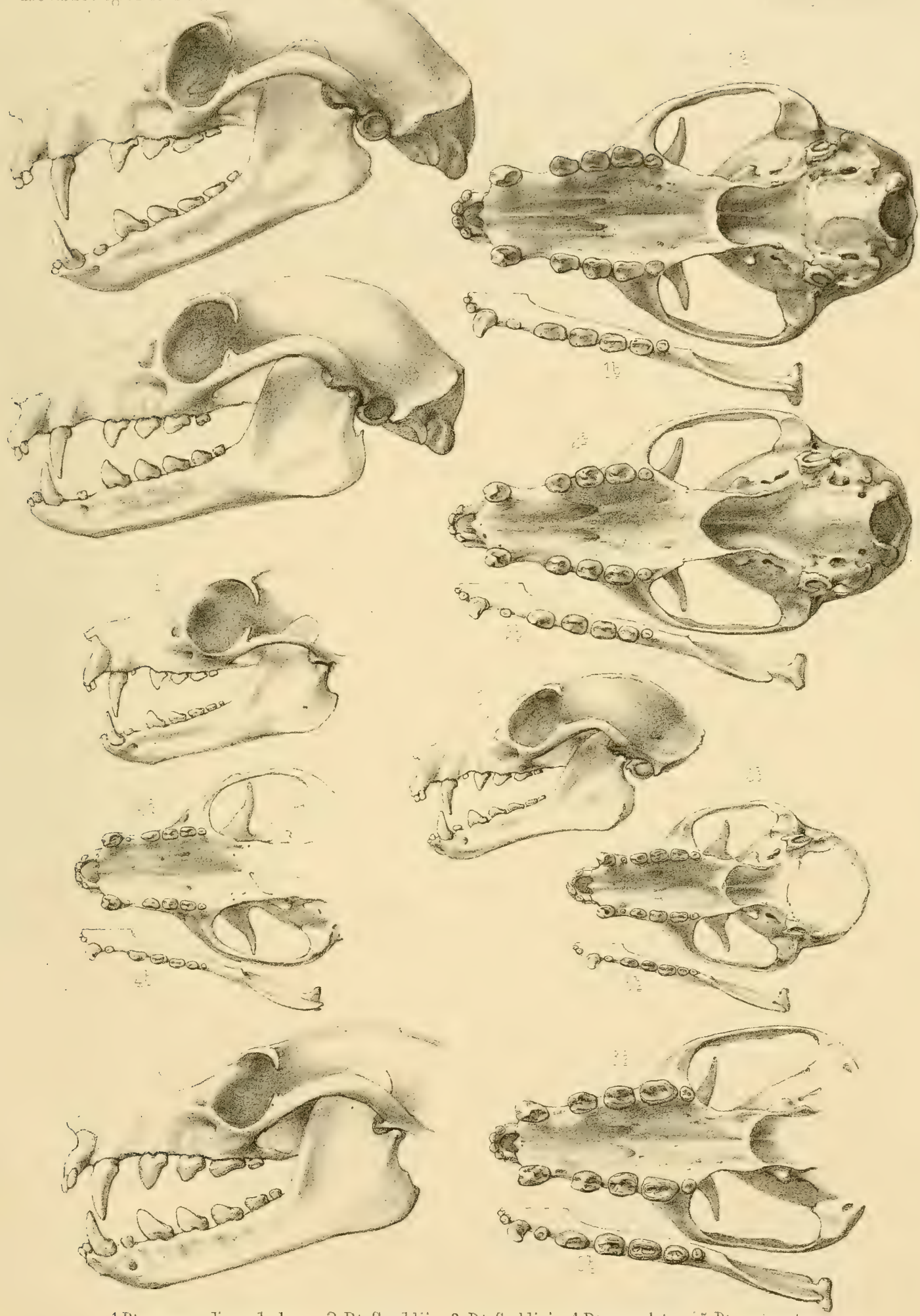

1.Pteropus poliocephalus: 2. Pt.Gouldii. 3. Pt.Geddiei. 4. Pt.scapulatus. 5. Pt. personatus. 


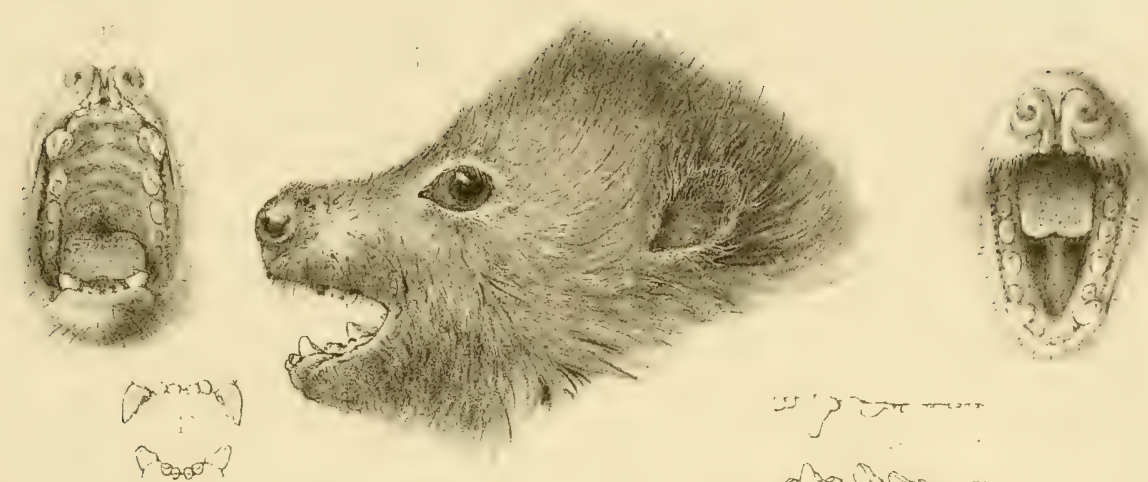

$(7 x+10)$

个erot?
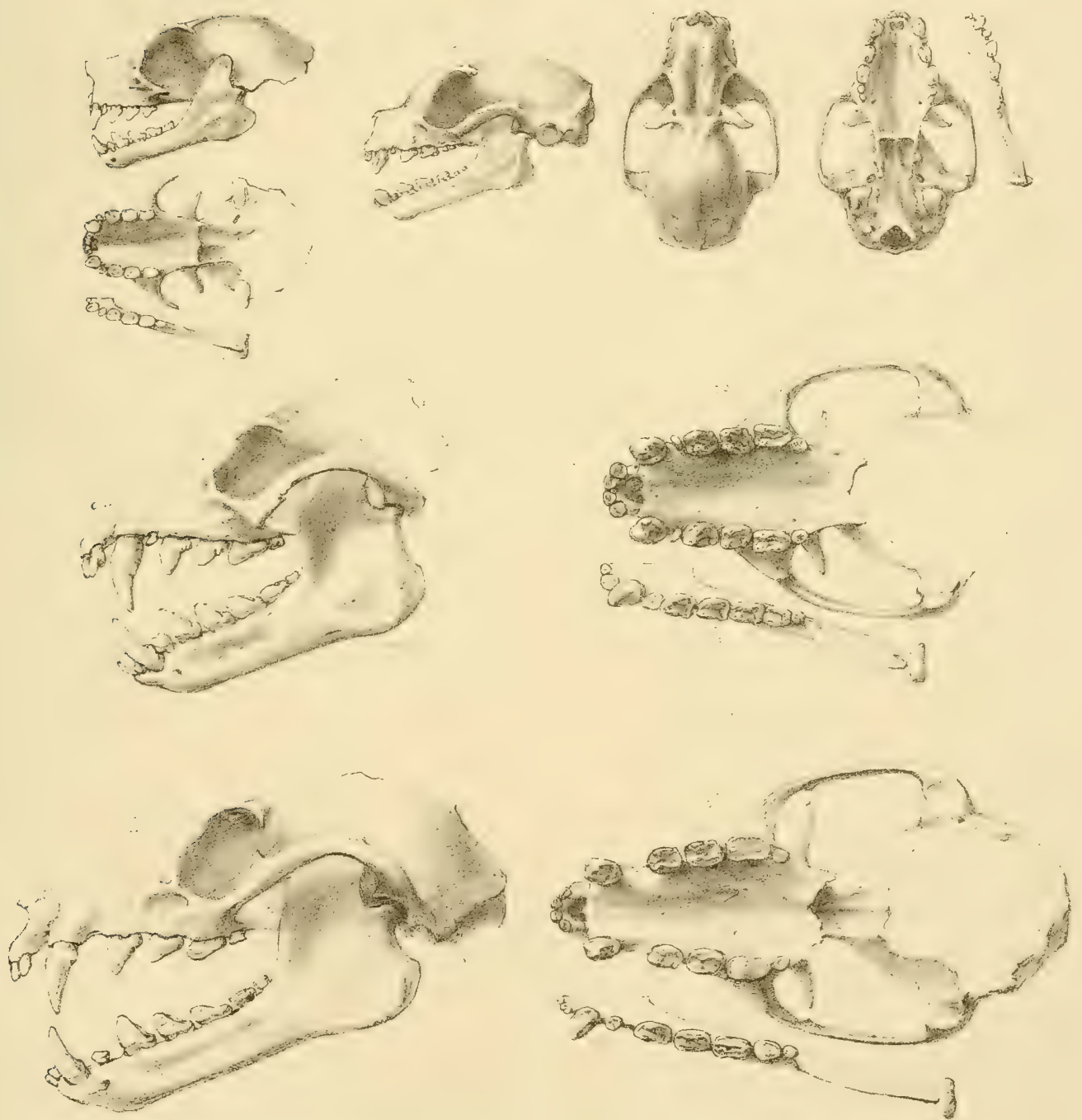

1.Pteropus rubricollis.2.Pt.condorensis.3.Putuberculatus.f. Cimonycteris Grandidieri. j.llegaerops ecaudatus 


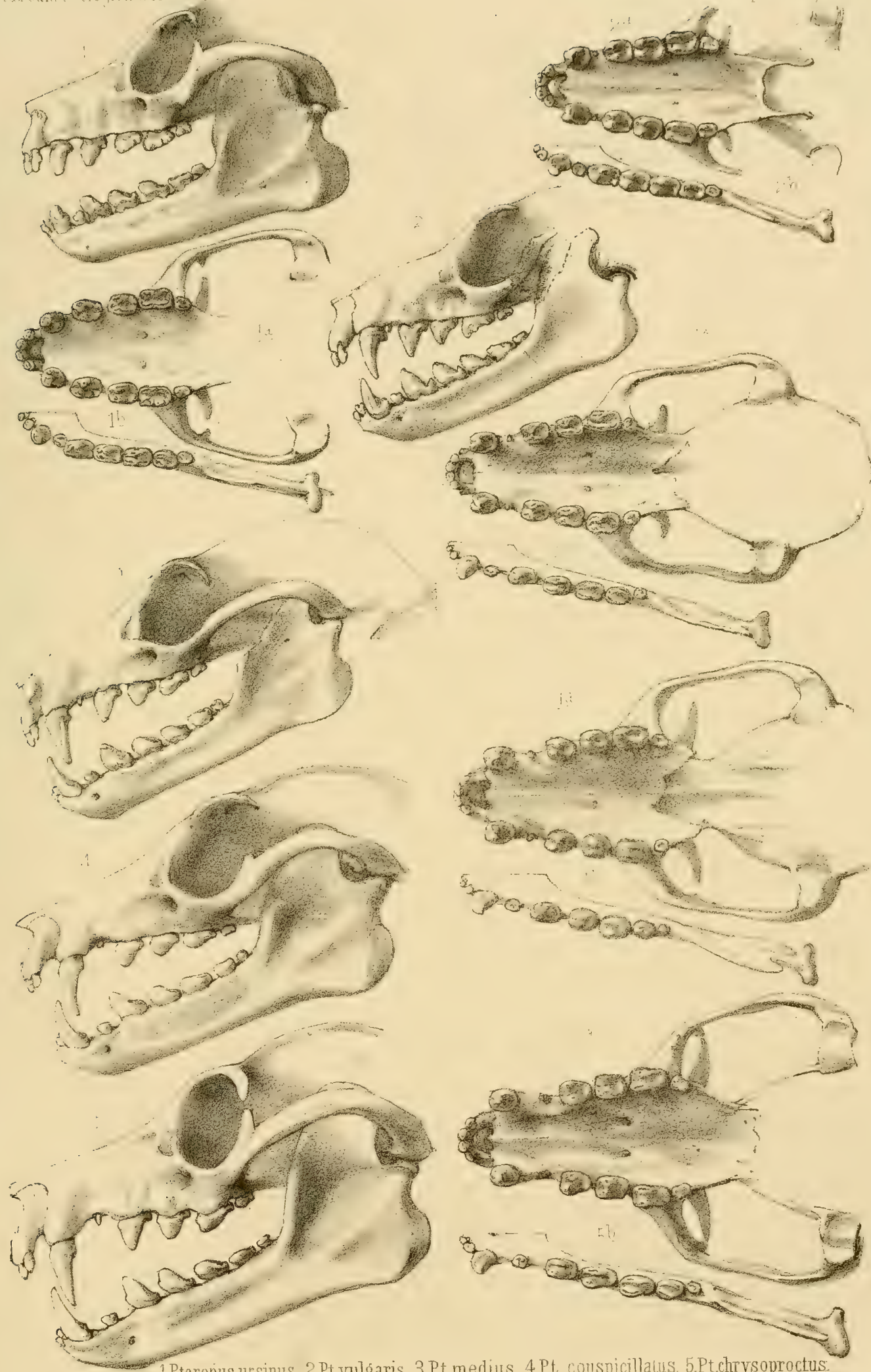


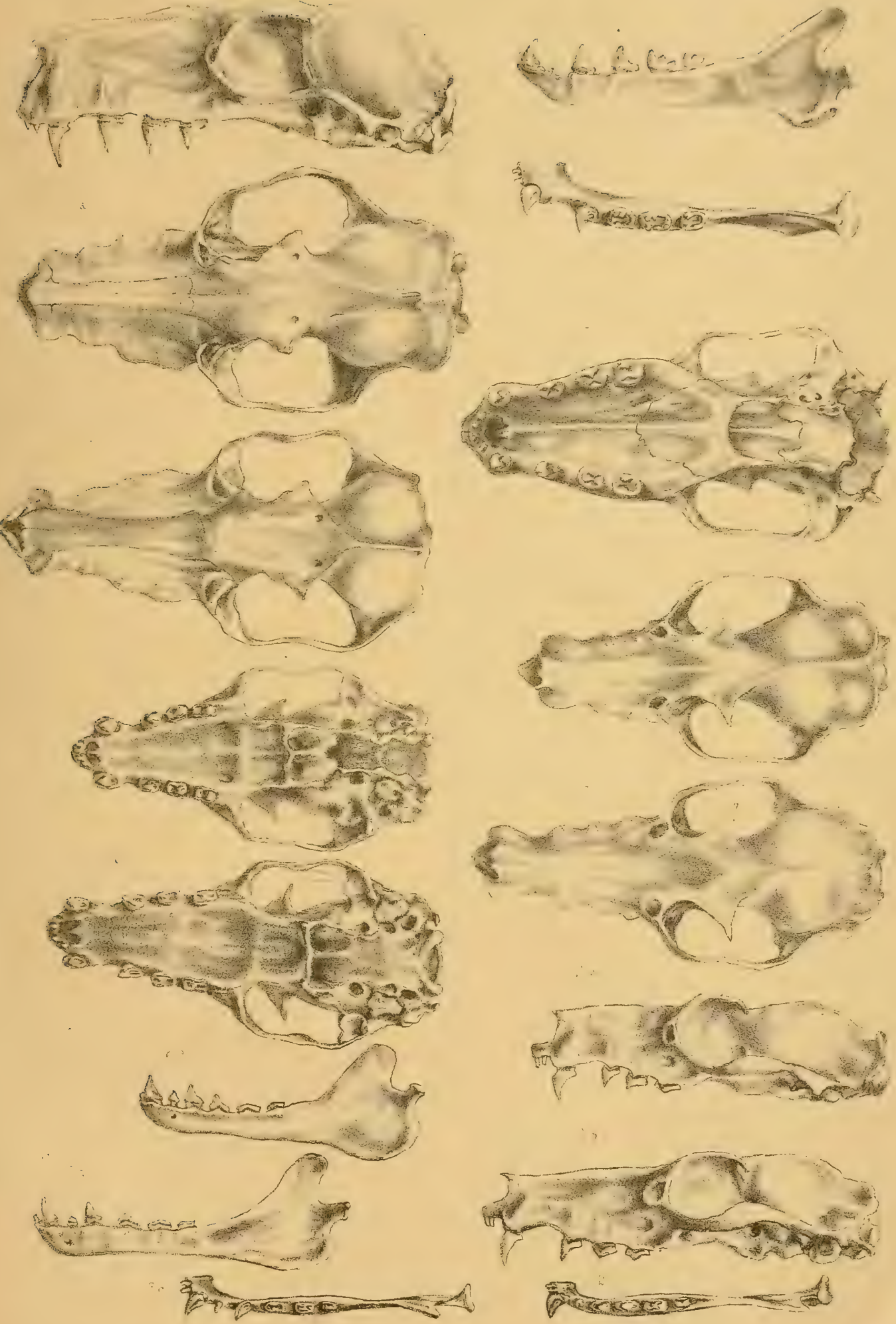

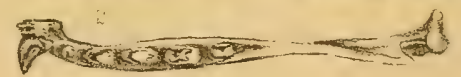



$1 \mathrm{a}$.
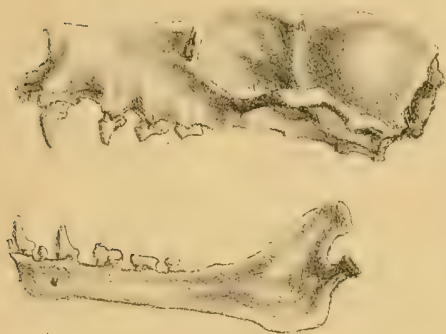

$1 \mathrm{~b}$.
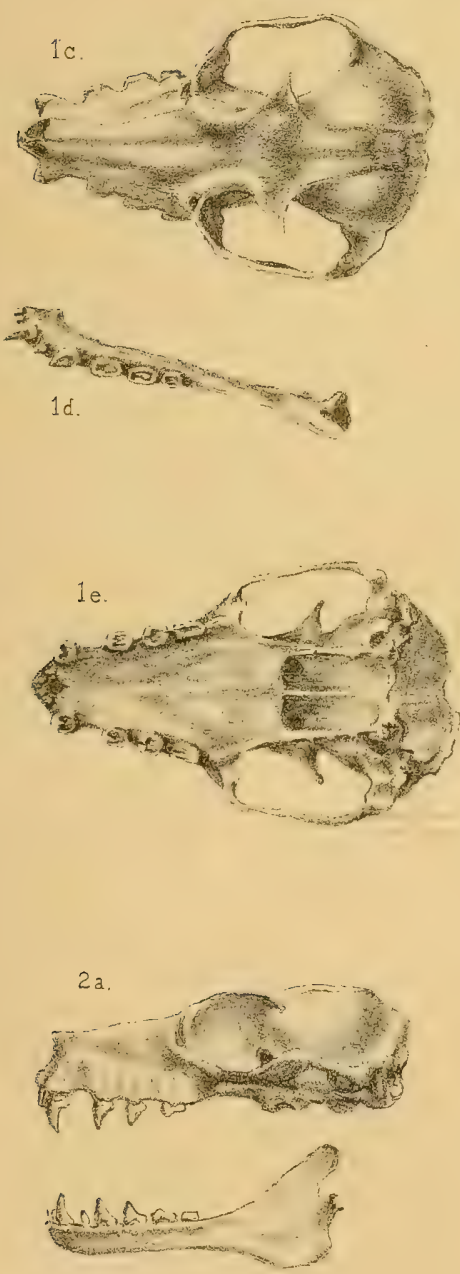

$2 b$.
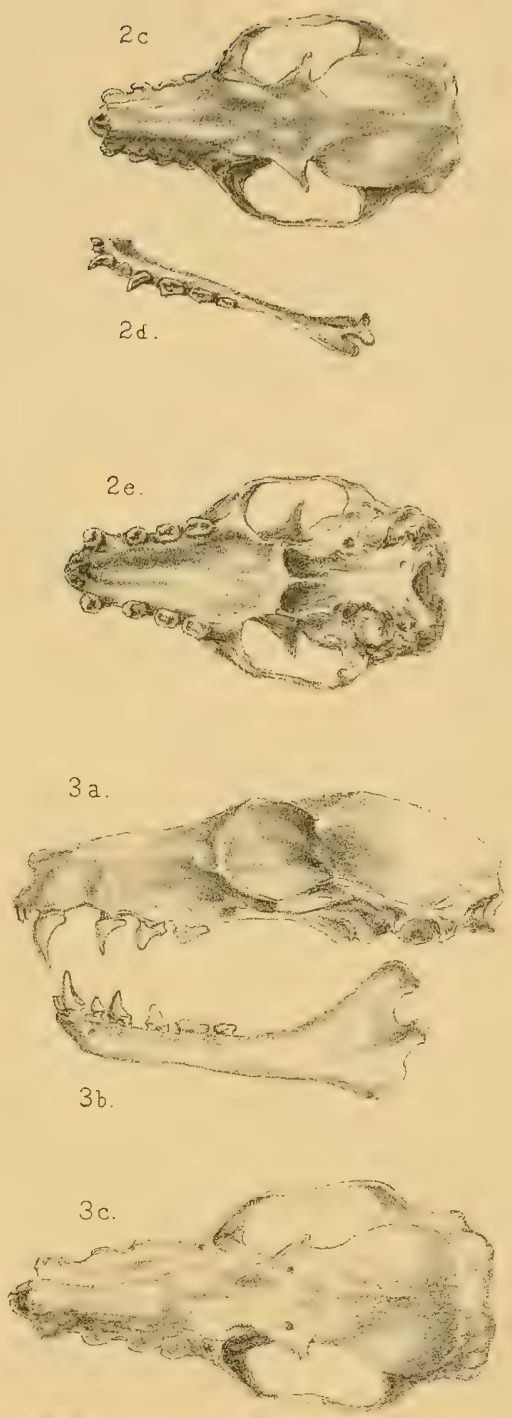

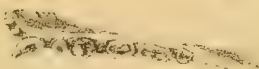

$3 d$
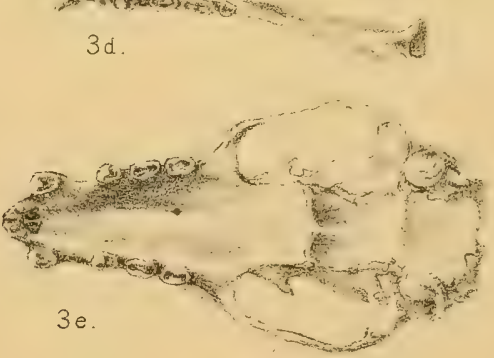

1.Epomophorus comptus. 2.Epomophorus minor. 3.Epomophorus crypturus.

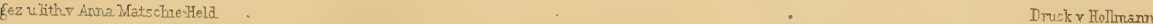





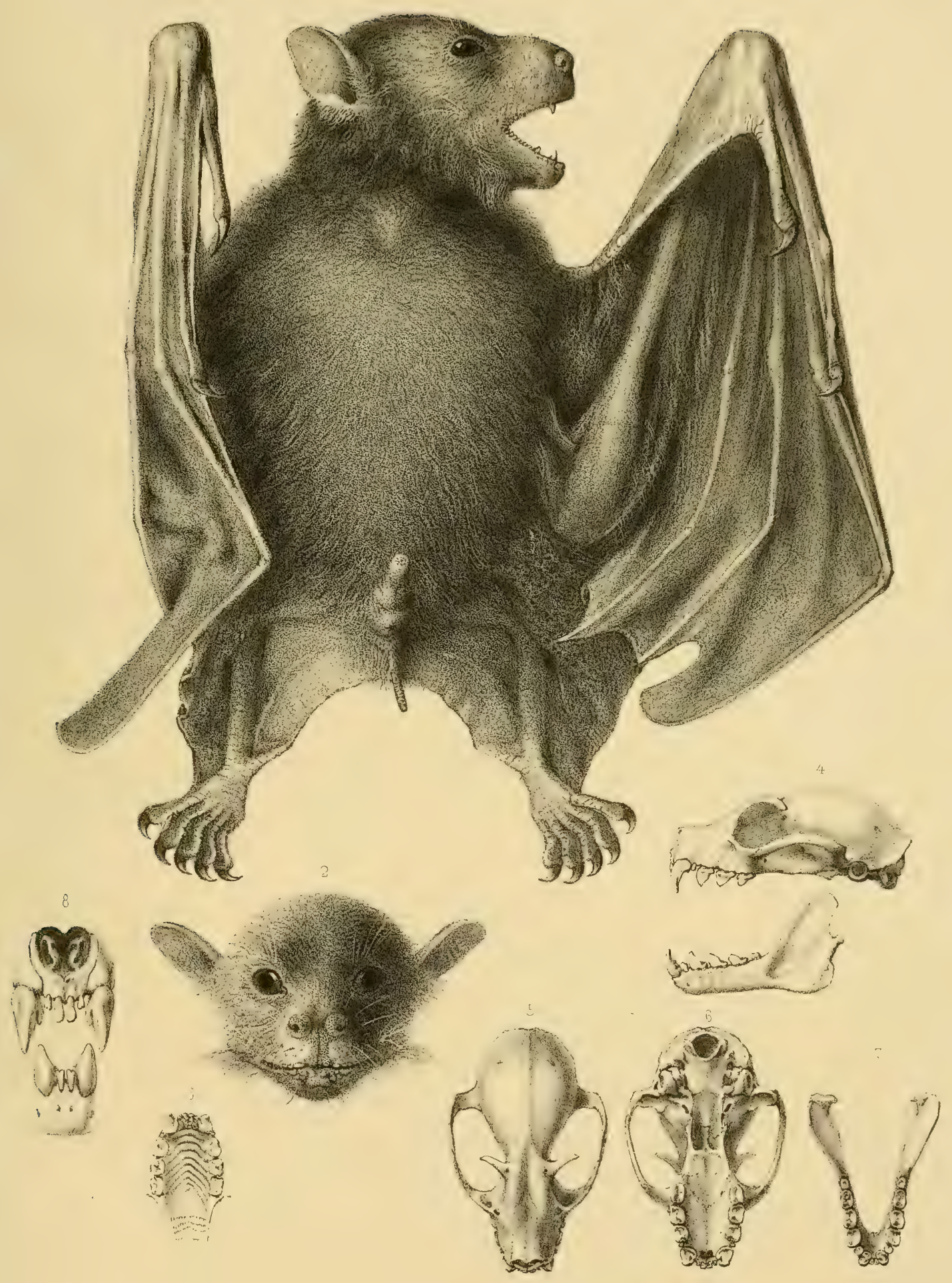

Crnopterus (Ptenochirus) Jag̉orii Peters 



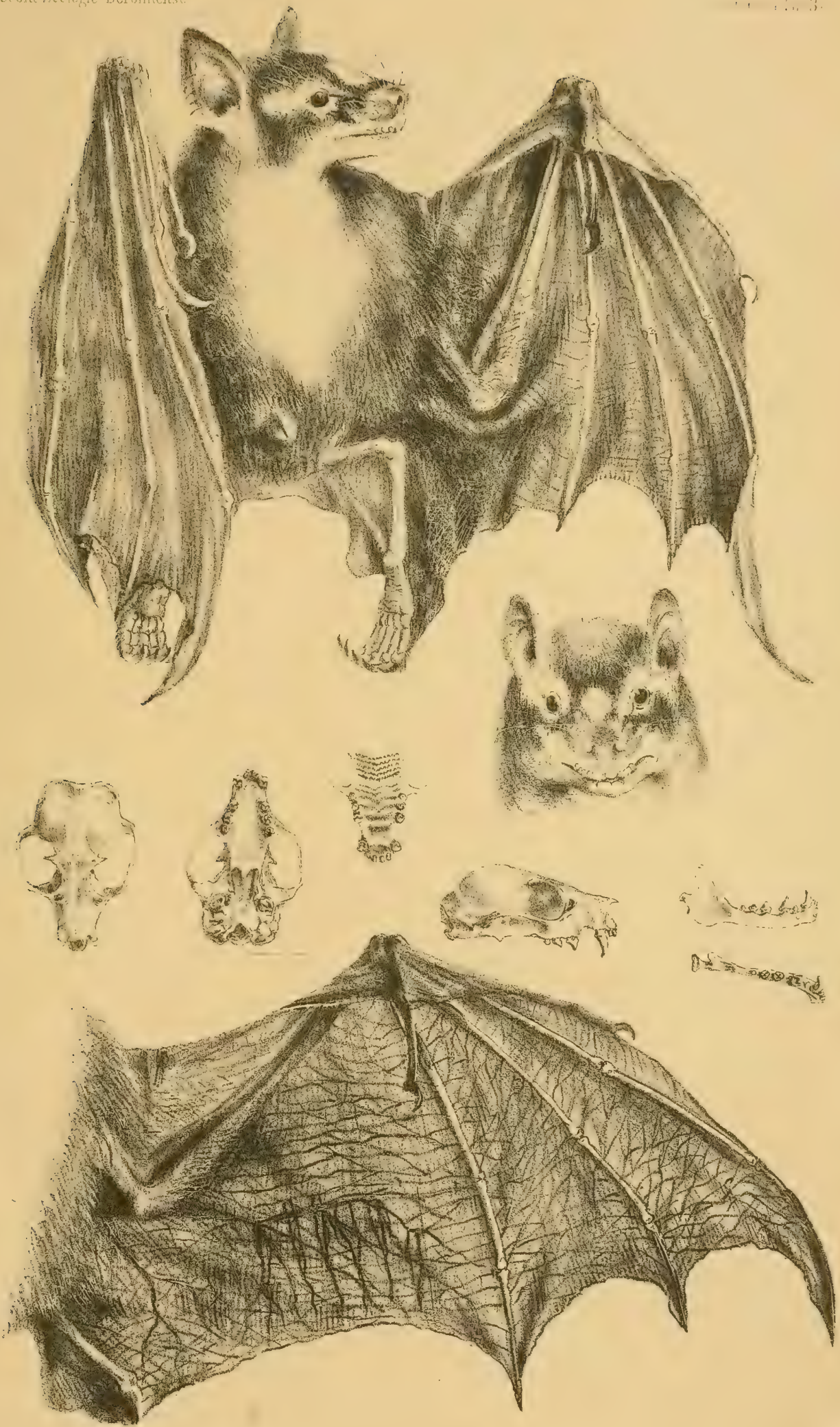




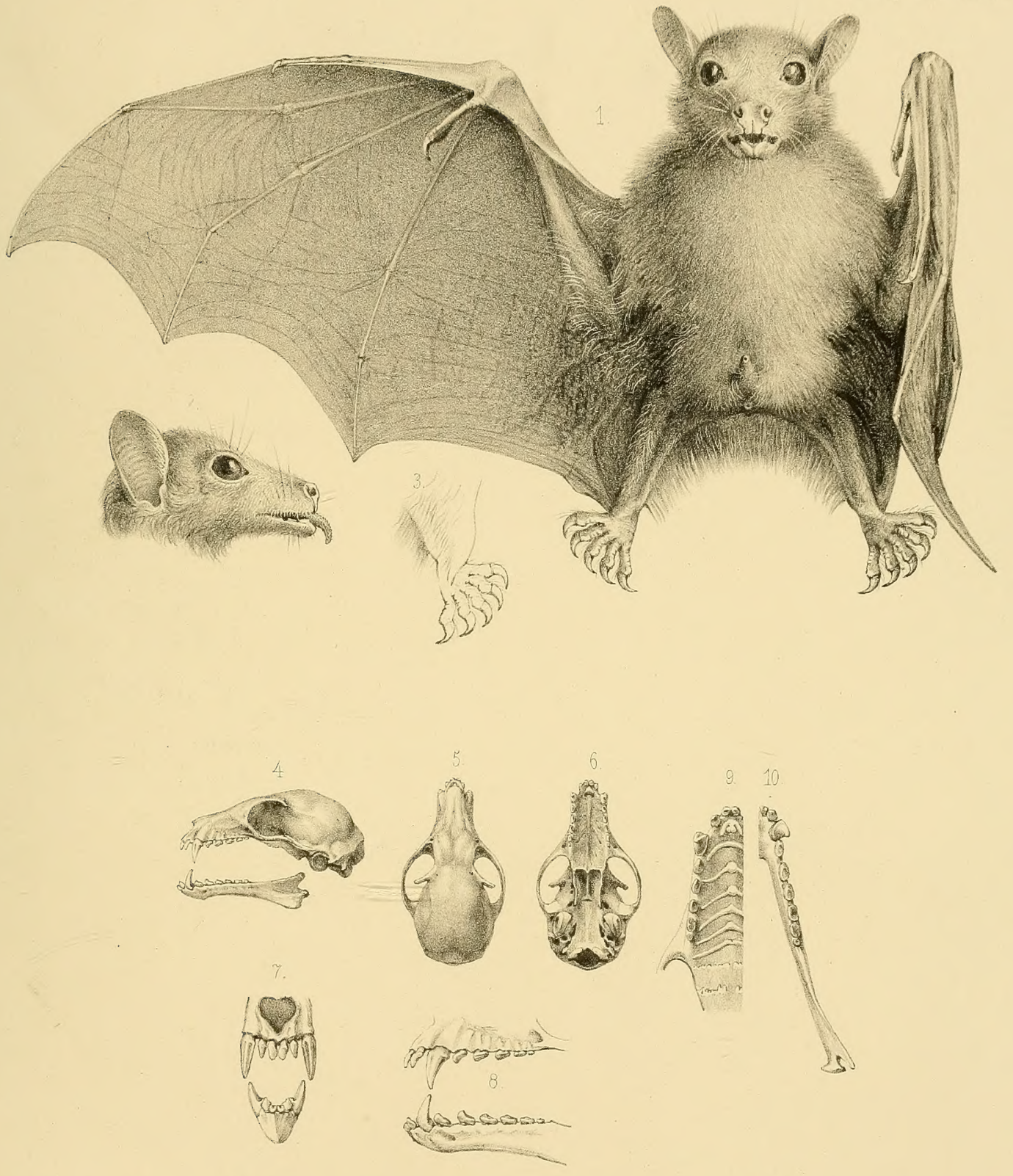

Macroǵlossus australis. 


